\title{
Elektronische Wertpapiere
}

\author{
Herausgegeben von \\ SEBASTIAN OMLOR, \\ FLORIAN MÖSLEIN und \\ STEFAN GRUNDMANN
}

Schriften zum

Recht der Digitalisierung

8

Mohr Siebeck 


\title{
Schriften zum Recht der Digitalisierung
}

\author{
Herausgegeben von \\ Florian Möslein, Sebastian Omlor und Martin Will
}

8

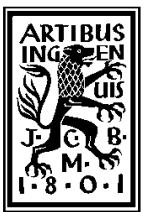





\title{
Elektronische Wertpapiere
}

\author{
Herausgegeben von \\ Sebastian Omlor, Florian Möslein \\ und Stefan Grundmann
}

Mohr Siebeck 
Sebastian Omlor ist Professor für Bürgerliches Recht, Handels- und Wirtschaftsrecht, Bankrecht und Rechtsvergleichung sowie Gründungsdirektor des Instituts für das Recht der Digitalisierung (IRDi) an der Philipps-Universität Marburg.

Florian Möslein ist Professor für Bürgerliches Recht, Deutsches und Europäisches Wirtschaftsrecht sowie Gründungsdirektor des Instituts für das Recht der Digitalisierung (IRDi) an der Philipps-Universität Marburg.

orcid.org/0000-0002-6733-6102

Stefan Grundmann ist Professor für Bürgerliches Recht, Deutsches und Europäisches Handels- und Wirtschaftsrecht an der Humboldt-Universität zu Berlin und Professor of Transnational Law and Theory am European University Institute, Florenz.

ISBN 978-3-16-160743-1 / eISBN 978-3-16-160744-8

DOI 10.1628/978-3-16-160744-8

Die Deutsche Nationalbibliothek verzeichnet diese Publikation in der Deutschen Nationalbibliographie; detaillierte bibliographische Daten sind im Internet über http://dnb.dnb.de abrufbar.

(C) 2021 Mohr Siebeck Tübingen. www.mohrsiebeck.com

Dieses Werk ist lizenziert unter der Lizenz „Creative Commons Namensnennung - Nicht kommerziell - Keine Bearbeitungen 4.0 International“ (CC-BY-NC-ND 4.0). Eine vollständige Version des Lizenztextes findet sich unter: https://creativecommons.org/licenses/by-nc-nd/4.0/deed.de.

Jede Verwendung, die nicht von der oben genannten Lizenz umfasst ist, ist ohne Zustimmung des Verlags unzulässig und strafbar

Das Buch wurde von epline in Böblingen aus der Stempel Garamond gesetzt und von Gulde Druck in Tübingen auf alterungsbeständiges Werkdruckpapier gedruckt und gebunden.

Printed in Germany. 


\section{Vorwort \\ Sebastian Omlor, Florian Möslein und Stefan Grundmann}

Das Gesetz zur Einführung von elektronischen Wertpapieren ist ein Besonderes, lange angemahnt.

Man kann - und muss es auch - sehen als ein Gesetz, das Effizienzgewinne bringen soll und wird, weil es eine digitalisierte Praxis, die bereits existiert, ohne juristische Krücke abbilden wird und soll. Es könnte jedoch auch ein Gesetz werden - im ersten oder aber im zweiten Anlauf -, das selbständig Architektur bildet in einem auch in seinen Konzepten digitalisierten Effektenmarkt - etwas Vergleichbares wie das Wertpapierhandelsgesetz vor 25 Jahren für die Effektentransaktionen und alternative Effektenmärkte, das Hopt deswegen einmal das Grundgesetz des Effektenhandels nannte. Dass der Wertpapierhandel elektronisch erfolgt, mit dem High Frequency Trade sogar der Großteil seines Volumens ohne elektronische Abwicklung schlichtweg nicht erfolgen könnte, ist allen bewusst. Ebenso ist allen bewusst, dass seit der Einführung der treuhänderisch gehaltenen Globalurkunde sowie der Wertpapiersammelverwahrung eine Gestaltung vorliegt, die Vorteile sachenrechtlichen Denkens für den gesamten Effektenverkehr auch weiterhin nutzbar machen konnte und eine elektronische Abwicklung dennoch ermöglichte. Dafür bedurfte es keines Gesetzes zur Einführung von elektronischen Wertpapieren, das muss solch ein Gesetz jedoch ebenfalls weiterhin verbürgen.

Da Globalurkunden im Verbund mit Wertpapiersammelverwahrung bereits digitalisierungstauglich waren, konnte diese Gestaltung auch immer wieder EU-Vorgaben gerecht werden, namentlich denen der sog. EG/EU-Finalitäts-Richtlinie 98/26/EG (mit ihrem Regime der Verlässlichkeit und Endgültigkeit von Buchungen, und wichtigen Fortschreibungen in EMIR und Richtlinie 909/2014/EU). Der Internationalisierungs- und Europäisierungstest des deutschen Depotrechts war damit zugleich bestanden. Umgekehrt bedeutet dies, dass das Gesetz zur Einführung von elektronischen Wertpapieren kein Umsetzungsgesetz ist, keine EU-Vorgabe neu umsetzen muss. Es ergeht autonom. Die Digitalagenda im Privat-, Handels- und Wirtschaftsrecht ist jedoch eine ungleich breitere als nur die einer Einführung von elektronischen Wertpapieren - ein Bereich, in dem Deutschland sogar eher den Nachzügler bildet. 
Die Digitalagenda ist ungleich breiter und in fast allen anderen Teilen ist sie eine, die vor allem auf EU-Ebene entwickelt wurde und wird. Dort hat sie in jüngerer Zeit auch in der Tat diesen Namen erhalten, diese systematische Ausgestaltung und diese strategische Durchführung, alles fußend auf dem sog. Gesellschaftsrechtspaket (vgl. EU-Rechtsrahmen für die Mobilität von Unternehmen im Binnenmarkt [,Gesellschaftsrechtspaket“] zur Änderung der Richtlinie [EU] 2017/1132, Zustimmung des Europäischen Parlaments vom 18.4.2018, 2018/0113 [COD], 2018/0114 [COD], erhältlich unter https://ec.europa.eu/info/ publications/company-law-package_en], namentlich mit der sog. EU-DigitalRichtlinie [EU] 2019/1151, dazu sogleich). Diese EU-Agenda reicht jedoch weit zurück, vor den Erlass der als solches bezeichneten EU-Digital-Agenda selbst. Man denkt an die frühen Ansätze im Bereich des Handels- und Unternehmensregisterrechts schon Anfang des Jahrtausends, seiner Digitalisierung und Verknüpfung zu einem EU-Unternehmensregister. Man denkt dann heute an das Digitalpaket für das EU-Gesellschaftsrecht selbst (sog. EU-Digital-Richtlinie [EU] 2019/1151, die viele Teile der allgemeinen gesellschaftsrechtlichen Kodifikations-Richtlinie [EU] 2017/1132 im Hinblick auf den Einsatz digitaler Werkzeuge und Verfahren im Gesellschaftsrecht modifizierte). Dabei ist freilich zu bedenken, dass schon zuvor erst die Digitalisierung den wichtigsten Schritt in der internen Organisation im EU-Gesellschaftsrecht, nämlich die grenzüberschreitende, binnenmarktweite Ausübung der mitgliedschaftlichen Verwaltungsrechte, namentlich Stimmrechte, überhaupt ermöglichte (Aktionärsrechte-Richtlinien I und II). Das eigentliche Digitalpaket im EU-Gesellschaftsrecht galt der Vertiefung dieser Grundlagen, darüber hinaus vor allem auch dem Einsatz digitaler Formate bei Gründung und Strukturmaßnahmen. Das Gesellschaftsrecht bildet jedoch nur ein Teilstück des für ein Gesetz über elektronische Wertpapiere relevanten Bereichs. Man denkt im Zivil-, Handels- und Wirtschaftsrecht bei der Digitalisierung gleichermaßen an die Digitalagenda für Verträge, Märkte, Hybridformen, namentlich Plattformen. Diese Agenda reicht von der EU-Richtlinie (EU) Nr. 2019/770 für Digitale Inhalte über die EU-Richtlinie (EU) Nr. 2019/771 für Waren allgemein und deren (häufig sehr zentralen) digitalen Komponenten, etwa Steuerungseinheiten, bis hin heute zur Initiative zur Regulierung der zentralen Intermediäre, der Plattformen, ursprünglich mit der sog. Informationsgesellschafts-Richtlinie 2001/29/EG, im Fokus der gegenwärtigen Regulierungsbemühungen der sog. Digital Services Act (geplant als EU-VO), $\operatorname{COM(2020)~} 825$ final. Und auch in diesem Bereich reichen die ersten Schritte bis an den Anfang des Jahrtausends zurück, mit der Regelung des elektronischen Vertragsschlusses durch die E-Commerce-Richtlinie 2000/31/EG, und fußt die Entwicklung in einer breiten Digitalagenda (vgl. Mitteilung der Kommission an das europäische Parlament, den Rat, den europäischen Wirtschafts- und Sozialausschuss und den Ausschuss der Regionen; KOM[2010] 245 endgültig/2 - mit Unterdokumenten für einzelne Akte.). All 
dies - digitale Register, digitale Märkte und Abschlüsse, digitale Plattformen, digitale Inhalte und Verbürgungen der Rechte - ist Teil einer breiten, fast schon flächendeckenden EU-Digitalisierungsagenda.

Das Gesetz zur Einführung von elektronischen Wertpapieren ist demgegenüber ein Besonderes. Denn es entspringt dem autonomen Willen des deutschen Gesetzgebers. Es hat in den Initiativen des Deutschen Bundestages zur Stärkung der Grundlagen von Digitalisierung sogar einen wichtigen Ursprung im Herzen des parlamentarischen Gesetzgebungsprozesses, des obersten Gesetzgebungsorgans selbst - nicht nur in Regierungsinitiativen und schon gar nicht nur auf Europäischer Ebene. Es handelt sich also um ein autonom gestaltetes Gesetz - eine Insel im Meer der Europäischen Digitalisierungsagenda. Und es ist besonders außerdem auch in dem Punkte, dass gerade die Gestaltung durch Globalurkunde und Wertpapiersammelverwahrung immer als eine besonders krude „Krücke“ gesehen und von allen führenden Bank- und Depotrechtlern als solche gegeißelt wurde (i. d. R. mit Hinweis auf die zahlreichen Initiativen im Europäischen und globalen Umfeld, im Soft Law, Konventions- und Richtlinienrecht), und dies in einem Land, das auf seine rechtswissenschaftlich-systematische Tradition, seine Kodifikationen in Zivil-, Handels- und Wirtschaftsrecht (gerade wegen dieser Qualität) besonders stolz ist. Das Gesetz zur Einführung von elektronischen Wertpapieren hatte also auch im Effekten- und Depotrecht einen Standard anzustreben, der dem großen Geist von Pandektistik, aber auch Schuldrechtsmodernisierung oder Aktiengesetzbuch zumindest nahekommen sollte.

Für das Gesetz stellen sich daher vor allem drei Fragen:

Das Gesetz hat die Vorteile rein elektronischer Abwicklung - über die Ästhetik der dogmatisch stringenteren Ausgestaltung hinaus - möglichst weitgehend zu realisieren, d. h. echte Effizienzgewinne zu ermöglichen. Und in der Tat rückt die Begründung von Entwurf und Vorschlag gerade diese auch in den Mittelpunkt. Die präzisere und verlässlichere Abbildung der materiellrechtlich zutreffenden Berechtigung wäre in vielen Bereichen - vom Rechtserwerb über den Schutz der Werte gegen Drittzugriff in Vollstreckungssituationen, ggf. auch bei hoheitlichen Eingriffen, bis hin zum Aufsichts- oder gar Steuerrecht - potentiell ein signifikanter, teils sogar ein überragend bedeutsamer (Effizienz-) Gewinn. Ein anderer signifikanter Gewinn könnte - unabhängig von der Alternative Zentralregister oder dezentrale Registergestaltung - in der größeren Verlässlichkeit, verbesserten Publizitätswirkung und gesteigerten Fälschungssicherheit liegen. Beide Punkte erörtert zentral - und mit substantieller Kritik Mathias Habersack.

Das Gesetz hat eine Architektur zu schaffen und dabei insbesondere der Vielzahl an Anlageinstrumenten eine klare depotrechtliche Ordnung zu geben - wobei die Ausklammerung von Anteilspapieren hinter dieser Zielvorstellung jedenfalls zurückbleibt. Kernziel müsste eines der Ordnung sein und dieses 
namentlich in zweierlei Hinsicht: bezogen auf die Instrumente und bezogen auf die Register, in denen sie erfasst sind. Bezogen auf die Instrumente wäre eine Ordnung zentral wichtig, die im Hinblick auf Zirkulationsfähigkeit, Standardisierungsklarheit und Wichtigkeit für den Effektenverkehr überzeugt. Zentrale Kriterien arbeitet Mathias Lehmann heraus, Florian Möslein vor allem auch, wie ein Provisorium (unter Ausklammerung der Anteilspapiere) dennoch Pfadabhängigkeiten schaffen kann, solchermaßen eher Rückschritt bedeutet. Ein wichtiger Aspekt wäre hier, dass im Spannungsverhältnis zwischen Inhaberund Namensaktien nicht eine Entwicklung gegenüber der anderen behindert werden sollte, wenn beispielsweise aus Governance-Überlegungen heraus der Trend hin zur Namensaktie Vorteile hat (gestalterische Neutralität). Bezogen auf die Register stellt sich zentral die Frage, welche Werte besser in Zentralregistern erfasst werden - und dann auch so, dass die Berechtigungen transparent werden - und welche besser in dezentralen Registern für Kryptowerte - wobei die Überlegung, dass diese noch am Anfang der Diskussion stehen, Gewicht hat und daher eine Festschreibung zum gegenwärtigen Zeitpunkt anders bewertet werden kann als bei den Zentralregistern, für die eine lange Erfahrung vorliegt und daher eine ausgereifte Lösung zu erwarten ist.

Eine dritte zentrale Frage - wiederum zur Gesamtarchitektur, jetzt jedoch noch stärker inhaltlicher Art - zielt auf die Leitbilder ab. Hierbei zentral ist zunächst die Frage nach der Ausgestaltung nach sachenrechtlichen Prinzipien, die nach einhelliger Meinung fortgeschrieben werden soll, für die sich jedoch vor allem die Alternative eröffnet, ob jeweils die mobiliarsachenrechtlichen, stärker an Übertragungsketten orientierten Wertungsprinzipien den Ausschlag geben oder eher die immobiliarsachenrechtlichen mit ihrer stärkeren Betonung der Registerverlässlichkeit und -dominanz - oder auch, in manchen Fragen jenes und in anderen wiederum dieses. Hierher zählt auch die Klärung der Bezüge zur allgemeinen Rechtsgeschäftslehre, namentlich mit der Frage, welche Verfügungshindernisse sinnvoll in einem Register vermerkt werden, welche nicht (und ggf. unabhängig davon durchgesetzt werden sollten). Die Referate von Omlor und Lieder zeigen in diesem Bereich Optimierungs- und Glättungspotential immer wieder auf. Ähnlich wichtig und für die Praxis wohl sogar überragend wichtig ist eine zweite Unterscheidung, namentlich zwischen Einzel- und Sammelverwahrung. Vor allem Einsele weist überzeugend darauf hin, dass die Einzelverwahrung zwar das gesetzgeberische Leitbild bildet, an das die Gestaltung der Sammelverwahrung durch umfangreiche Verweise anknüpft, jene jedoch in der praktischen Wichtigkeit hinter dieser weit zurücksteht. Umso problematischer ist jede Gestaltung im neuen Gesetz, das Konstellationen der Sammelverwahrung nicht gleichwertig mit einbezieht und vor allem diese überzeugend durchgestaltet.

Alles andere als eine zügige Verabschiedung des Gesetzes wäre eine Überraschung. $\mathrm{Ob}$ es noch gelingt, in manchem Teil des Gesamtgerüsts zentrale 
Petita zu verankern, ist die vielleicht wichtigste verbleibende Frage - weil man sich nicht leichtfertig auf eine Korrektur des Provisoriums verlassen sollte. Drei der wichtigsten Petita sind: die Abrundung des Anwendungsbereichs, etwa im Hinblick auf Mitgliedschaftsrechte, aber auch hybride Instrumente; die Klärung der (sachenrechtlichen) Vorbilder, die Vermutungen, Rechteinhaberschaft und -übertragung leiten; und die Schaffung noch größerer Transparenz bei der Rechteinhaberschaft. Die Schweizerische Regelung, vorgestellt durch Zellweger-Gutknecht, mag noch stärker als Vorbild berücksichtigt werden. 



\section{Geleitwort}

\section{Christine Lambrecht}

Blockchain und Recht? Es ist noch nicht lange her, da galt das vielen als randständiges Thema. Doch inzwischen haben sich die Verhältnisse gewandelt. Der vorliegende Band belegt das eindrücklich. Er behandelt ein aktuelles und ambitioniertes Gesetzgebungsvorhaben aus der Blockchainstrategie der Bundesregierung, die Einführung von elektronischen Wertpapieren; er versammelt ein breites Spektrum an Expertinnen und Experten; und er steht in Zusammenhang mit einem von meinem Haus geförderten Forschungsvorhaben, dem Projekt „Blockchain und Recht“ an der Philipps-Universität Marburg. All das unterstreicht: Das Thema Digitalisierung ist in der Mitte von Rechtspolitik und -wissenschaft angekommen. Und damit dort, wo es hingehört.

Die Einführung von elektronischen Wertpapieren und die in diesem Band versammelten Beiträge zeigen auch: Das Recht an die digitale Entwicklung anzupassen ist eine anspruchsvolle Aufgabe. Natürlich leuchtet es heute nicht mehr ein, dass Wertpapiere in einer Papierurkunde verbrieft werden müssen. Aber mit dieser Einsicht allein ist noch nicht viel gewonnen. Denn gerade bei einer so anspruchsvollen Materie wie dem Recht der Finanzinstrumente steckt der Teufel im Detail. Was ist zu tun, damit elektronische Wertpapiere am Kapitalmarkt genauso viel Vertrauen genießen wie Papierurkunden? Wie lassen sich Effizienz im Handelsverkehr und ein starker Anlegerschutz verbinden? Wie müssen die neuen Regelungen aussehen, damit sie sich stimmig in unsere Rechtsordnung einfügen? Und, in diesem dynamischen Umfeld besonders wichtig: Wie öffnen wir das Recht für technologische Innovationen? Das sind nur einige der Fragen, die bei der Einführung von elektronischen Wertpapieren zu beantworten sind. Und schon sie machen deutlich, wie groß die Aufgabe ist.

Das Gesetz zur Einführung von elektronischen Wertpapieren wird erstmals die elektronische Begebung von Schuldverschreibungen und von Fondsanteilscheinen ermöglichen. Damit wird das Gesetz - so viel lässt sich heute schon absehen - nicht den Endpunkt der Modernisierung des Wertpapierrechts markieren. Denn natürlich gilt auch für Aktien und andere Wertpapiere: Eine zwingende Verbriefung ist nicht länger zeitgemäß. Bevor auch für diese Wertpapiere eine elektronische Form vorgesehen werden kann, sind allerdings noch weitere, anspruchsvolle Fragen zu klären. Und das wird nur gelingen, wenn sich 
Wissenschaft, Technik, Rechtspraxis und Politik eng austauschen - so wie das im Rahmen der Tagung geschehen ist, auf die der vorliegende Band zurückgeht.

Den Veranstaltern der Tagung - dem Institut für das Recht der Digitalisierung (IRDi) an der Philipps-Universität Marburg und dem Institut für Bankund Kapitalmarktrecht an der Humboldt Universität zu Berlin, den Herren Professoren Omlor, Möslein und Grundmann und ihren Mitarbeiterinnen und Mitarbeitern - gilt mein herzlicher Dank. Möge dem vorliegenden Band die Aufmerksamkeit zuteilwerden, die er verdient. Und mögen Wissenschaft, Politik und Gesellschaft bei der Bewältigung der Herausforderung der Digitalisierung weiterhin produktiv zusammenarbeiten! 


\section{Inhaltsverzeichnis}

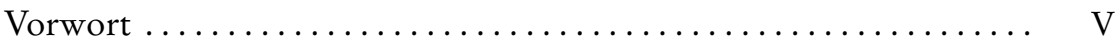

Geleitwort $\ldots \ldots \ldots \ldots \ldots \ldots \ldots \ldots \ldots \ldots \ldots \ldots \ldots \ldots \ldots \ldots \ldots \ldots \ldots \ldots, \quad$ XI

Teil 1: Einordnung

Editorial: Das eWpG im digitalen Aufbruch

des Kapitalmarkt- und Gesellschaftsrechts

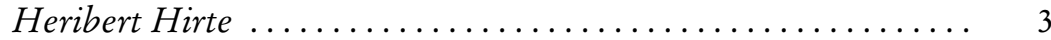

$\$ 1$ Internationaler Kontext: Schweizerisches Registerwertrecht

Corinne Zellweger-Gutknecht/Lucien Monnerat .............

Teil 2: Regelungsvorschläge

$\$ 2$ Die allgemeinen privatrechtlichen Regelungen für elektronische Wertpapiere. National und international

Dorothee Einsele ................................. 33

$\$ 3$ Wertpapierarten

Matthias Lebmann ............................... 59

$\$ 4$ Zentrales Register und Kryptowertpapierregister

Mathias Habersack ............................. 83

\5 Eintragung und Publizität elektronischer Wertpapiere Jan Lieder ...................................... 103

\$6 Verfügungen über elektronische Wertpapiere Sebastian Omlor............................... 137

$\$ 7$ Aufsicht und Sanktionen Julia von Buttlar................................. 157 
Teil 3: Ausblick

\8 Elektronische Geschäftsanteile

Florian Möslein.................................... 179

$\$ 9$ Elektronische Fondsanteile

Thomas Eckhold................................... 207

Autorenverzeichnis .............................. 225 
Teil 1

Einordnung 



\title{
Editorial \\ Das eWpG im digitalen Aufbruch des Kapitalmarkt- und Gesellschaftsrechts
}

\author{
Heribert Hirte
}

Das Computerrecht, später dann das Informationsrecht, fristete lange ein Nischendasein. Die sprunghafte Steigerung der Rechenleistung und damit einhergehende Entwicklungen wie die Distributed-Ledger-Technologie (DLT) oder Künstliche Intelligenz insbesondere mittels Machine Learning sprengen aber die Grenzen des bislang Denkbaren. Die sogenannte zweite Welle der Digitalisierung führt heute zu Diskussionen und neuen Fragen in allen Bereichen des Rechts, ohne dass sie sich noch auf ein bestimmtes Rechtsgebiet wie das Computerrecht isolieren ließen. Zeitgleich unterliegt der Kapitalmarkt einem großen digitalen Wandel. Es werden FinTechs gegründet und neue finanztechnische Geschäftsideen entwickelt, die Abwicklung von Bankgeschäften wird automatisiert, im Hochfrequenzhandel ist kaum noch ein Mensch eingebunden. Dadurch stehen herkömmliche Banken und Finanzdienstleister vor großen Herausforderungen. Welche Auswirkungen die digitale Vernetzung und Abstimmung an den Finanzmärkten hervorbringen, konnten wir an der Causa Gamestop beobachten.

Die Digitalisierung der Finanzbranche ist zugleich auch Aufgabe für Politik und Gesetzgebung. Der moderne und effizienzgetriebene Kapitalmarkt bietet Chancen, verlangt aber auch nach Antworten auf seine Risiken. Das Eckpunktepapier der CDU/CSU-Bundestagsfraktion zur Blockchain verweist bereits auf das Erfordernis eines agilen Vorgehens des Gesetzgebers. Dabei wollen CDU und CSU schrittweise Gesetzesveränderungen durchführen, die Innovationen in Deutschland unterstützen, ohne bewährte rechtliche Prinzipien aufzugeben. Es gilt, sinnvolle Anwendungsfelder neuer Technologien zu identifizieren und Innovationen zu fördern. Dies gelingt zum einen durch die Schaffung einer innovationsfreundlichen Gesetzeslage und zum anderen durch eigene Kompetenz in digitalen Themen. Die Bundesregierung setzte sich in ihrer Blockchain-Strategie das Ziel, das deutsche Recht für elektronische Wertpapiere zu öffnen und startete mit einem Eckpunktepapier für die regulatorische Behandlung von elektronischen Wertpapieren den Konsultationsprozess. 
Das Gesetz zur Einführung von elektronischen Wertpapieren sieht vor, mittels Eintragung in ein elektronisches Wertpapierregister die Begebung von elektronischen Schuldverschreibungen und Anteilsscheinen zu ermöglichen. Das kann und soll allerdings nur ein erster Schritt sein, denn elektronische Gesellschaftsanteile wie die E-Aktie oder der elektronische $\mathrm{GmbH}$-Anteil werden mit dem Gesetz nicht eingeführt. Sie sind der nächste logische Schritt und wären vor allem auch für Kapitalgesellschaften jenseits der Börsennotierung und Start-ups eine Erleichterung. Sie würden unabhängig von der neuen Online-Gründung die Gründung von Kapitalgesellschaften erleichtern und durch eine günstigere Übertragbarkeit der Aktien und Anteile die Eigenkapitalbeschaffung und die Mitarbeiterkapitalbeteiligung vereinfachen. Hier wird es in der nächsten Legislaturperiode darauf ankommen, am Ball zu bleiben, und das Tempo der digitalen Erneuerung von Kapitalmarkt und Gesellschaftsrecht noch zu erhöhen.

Deutschland kann hier aber nicht autonom agieren. Es ist auf die Konsistenz mit europarechtlichen Vorgaben und Initiativen in diesem Bereich zu achten. Die aufsichtsrechtlichen Regelungen werden nicht weniger und durch MiFID II und MiFIR noch komplexer. Das eWpG wird mit den Ideen der europäischen Krypto-Regulierung im MiCAR harmonieren müssen. Für die Finanzmärkte ist es elementar, keine Bruchstellen entstehen zu lassen, die in Folge den Finanzstandort Deutschland behindern würden. Schließlich kommt es maßgeblich auf das Zusammenspiel und das Verständnis von Gesellschaftsrecht, nationalem und europäischem Kapitalmarktrecht und digitalen Techniken an. Im Hinblick auf einen agilen und modernen Regulierungsansatz sei auf das DLT Pilot Regime der EU verwiesen, das regulatorische Ausnahmen ermöglicht, um Pilotprojekte für die Nutzung von Distributed-Ledger-Technologie zu fördern.

Wenn man den Blick zum Abschluss noch etwas weiter schweifen lässt, blicken wir unmittelbar auch auf den digitalen Euro. Nach einer aktuellen Studie denken bereits 86 Prozent der befragten Zentralbanken über eigene digitale Zentralbankwährungen nach. In China und Schweden laufen bereits Pilotphasen, die EZB befindet sich dagegen noch in einem sehr frühen Stadium, möchte aber Mitte 2021 über den Start des Projekts entscheiden. Der digitale Euro könnte den Zahlungsverkehr im Euroraum erheblich beschleunigen und in manchen Fällen auch vollständig automatisieren. Somit würde er Innovationen im Massenzahlungsverkehr aktiv fördern und dadurch, dass Geschäftsbanken nur als Mittler zwischen EZB und dem Inhaber des E-Euros dienen würden, auch im Vergleich zu ApplePay oder GooglePay für mehr Vertrauen und Sicherheit im Zahlungsverkehr sorgen. Zu beachten ist allerdings, dass die Einführung digitalen Zentralbankgelds mit erheblichem Kostenaufwand verbunden ist. Aus diesem Grund haben sich die Schweiz und Dänemark gegen dessen Einführung entschieden. Auf die Entscheidung der EZB und deren Folgen kann man gespannt sein. 
Es zeigt sich, dass die nahe Zukunft viele spannende Herausforderungen im Bereich der Digitalisierung des Kapitalmarkts und der damit zusammenhängenden Rechtsgebiete bereithält. Der Gesetzgeber, aber auch der Autor, stellen sich den damit zusammenhängenden Fragen nicht erst seit dem eWpG. Die Herausforderungen werden zwischen notwendiger Regulierung, um Rechtssicherheit und Vertrauen zu schaffen, sowie wichtiger Technologie- und Innovationsoffenheit nicht kleiner. Gerade deshalb ist auch in diesem Bereich ein enger Austausch zwischen der Wissenschaft, Innovationstreibern und dem Gesetzgeber erforderlich. Ich bin optimistisch, dass sich dieser Austausch auch über die Tagung zum eWpG und diesen Tagungsband hinaus fortsetzen wird. 



\section{Internationaler Kontext: Schweizerisches Registerwertrecht}

\section{Corinne Zellweger-Gutknecht/Lucien Monnerat*}

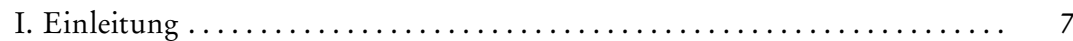

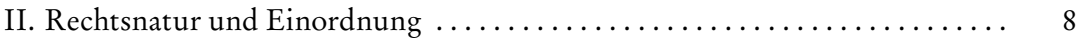

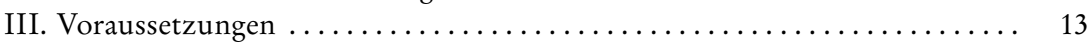

1. Registerwertrechtsfähige Rechte $\ldots \ldots \ldots \ldots \ldots \ldots \ldots \ldots \ldots \ldots \ldots \ldots$

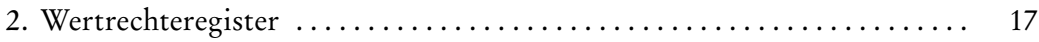

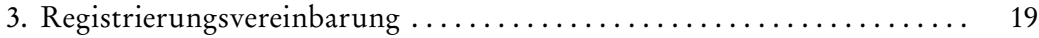

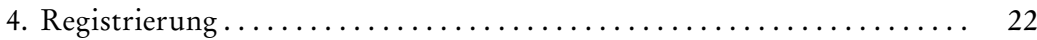

5. Weitere, nicht konstitutive Anforderungen $\ldots \ldots \ldots \ldots \ldots \ldots \ldots \ldots 22$

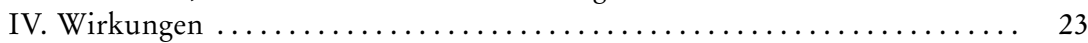

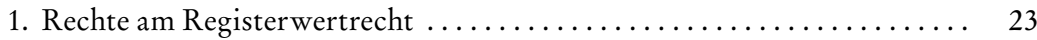

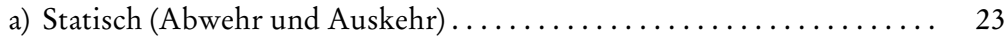

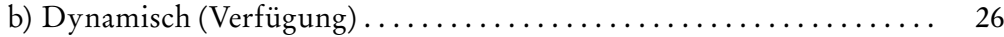

2. Rechte aus dem Registerwertrecht .................... 28

IV. Ausblick ..................................... 29

\section{Einleitung}

Zum 1. Februar bzw. zum 1. August 2021 ist das Schweizerische „Bundesgesetz zur Anpassung des Bundesrechts an Entwicklungen der Technik verteilter elektronischer Register" in Kraft getreten. ${ }^{1}$ Es handelt sich um ein reines Rahmengesetz, das zehn bestehende Bundesgesetze anpasst mit dem Ziel, eine sichere rechtliche Basis zu schaffen für den Handel von Rechten mittels Distributed-Ledger-Technologie (DLT), aber auch mittels heute noch unbekannter Technologien, die eine ausreichend manipulationsresistente Datenverarbeitung gewährleisten. ${ }^{2}$ Die Chancen, welche mit solchen neuen Technologien einhergehen, sollen künftig genutzt werden können, ohne dass korrespondierende

* Die Autoren danken Prof. Dr. iur. Rolf H. Weber, Rechtsanwalt, Zürich für wertvolle Hinweise.

1 Vgl. zu Entstehung und Inhalt im Einzelnen Zellweger-Gutknecht/Seiler, Country Report Switzerland, in: Maume/Maute(Hrsg.), The Law of Crypto Assets (erscheint demnächst).

2 Bundesrat, Botschaft zum Bundesgesetz zur Anpassung des Bundesrechts an Entwick- 
Risiken die Interessen der Akteure und die Integrität und Reputation des Finanz- und Wirtschaftsstandorts Schweiz beeinträchtigen. ${ }^{3}$ Schließlich sollte die rechtliche Basis ausreichend flexibel ausgestaltet werden, so dass sich die Schweiz als ein führender, innovativer und nachhaltiger Standort für Fintechund DLT-Unternehmen etablieren und weiterentwickeln kann. ${ }^{4}$ Einer der Dreh- und Angelpunkte des Rahmengesetzes bildet das neu geschaffene Registerwertrecht, das im Zentrum des vorliegenden Beitrages steht.

\section{Rechtsnatur und Einordnung}

Die neuen Bestimmungen zu den Registerwertrechten stehen in der fünften und letzten Abteilung des Obligationenrechts betreffend „Die Wertpapiere“ (Art. $965 \mathrm{ff} . \mathrm{OR}$ ) und dort, gleich eingangs, unter den Allgemeinen Bestimmungen (Art.965-973i OR) über die Namen-, Inhaber- und Orderpapiere (Art. 965-1155 OR). Indes wollte der Gesetzgeber das Registerwertrecht mit dieser systematischen Verortung nicht etwa als normativ fingiertes Wertpapier konzipieren. ${ }^{5}$ Vielmehr hat er ein eigenständiges, wenn auch wertpapierähnliches Instrument geschaffen.

Die Ähnlichkeit ist funktional bedingt: Beim Wertpapier verknüpft eine sog. einfache Wertpapierklausel ${ }^{6}$ Recht und Urkunde so, dass ohne Urkunde über das Recht nicht verfügt werden kann (Art. 965 OR); mithin folgt das Recht aus dem Wertpapier dem Recht am Wertpapier. Diese Verknüpfung führt punkto Transport, Legitimation und Verkehrsschutz zu Privilegien, welche durch Legitimationsklauseln ${ }^{7}$ noch vermehrt werden können und so die Umlauffähigkeit

lungen der Technik verteilter elektronischer Register, 27. November 2019, BBl. 2020, S. 233, 234.

${ }^{3}$ Die Botschaft (Fn. 2), S. 234 erwähnt zwar den Anlegerschutz nicht explizit. Zumindest einzelnen Normen des Rahmengesetzes sind indes eindeutig individualschützend ausgestaltet (so etwa Art. 973i OR zur Haftung). Zum technologieneutralen Ansatz etwa S. 259.

${ }^{4}$ Botschaft (Fn. 2), S. 239.

${ }^{5}$ Die Fiktion wäre notwendig, da die für das Wertpapier in Art. 965 OR vorausgesetzte Urkunde nach herrschender Lehre einen physischen Transfer gewährleisten muss: von der Crone/Derung, Aktien als digitalisierte Werte, SZW 5 (2019), 481, 483; MacCabe, Die Abbildung von Vermögenswerten durch Anlage-Tokens (2019), S. 6 ff.; Ifflan/Ben Hattar, Central Securities Depositaries in the Age of Tokenized Securities, CapLaw 5 (2020), $419 \mathrm{f}$.; Pasquier/ Ayer, Formungültige Aktienübertragungen auf der Blockchain, Anwaltsrevue 5 (2019), 196, 198; a. M. immerhin Weber/Iacangelo, Rechtsfragen bei der Übertragung von Token, Jusletter IT (24. Mai 2018), S. 10; Swiss Blockchain Federation (SBF), Stärkung des Blockchain-Standorts Schweiz, Whitepaper der Blockchain-Taskforce, (April 2018), S. $6 \mathrm{ff}$.

${ }^{6}$ Sog. doppelseitige Präsentationsklausel, dergemäss ein Schuldner ohne Vorlage der Urkunde weder leisten muss noch darf: vgl. schon Jäggi/Druey/von Greyerz, Wertpapierrecht unter besonderer Berücksichtigung von Wechsel und Check (1985), S. 79 (dort zum Sparheft).

7 Die einfache Legitimationsklausel erlaubt dem Schuldner, befreiend an den durch die Urkunde ausgewiesenen Gläubiger zu leisten (bei Orderpapier mit Blankoindossament und 
von Wertpapier und verbrieftem Recht (im Vergleich zu unverbrieften oder gar unverurkundeten Rechten $)^{8}$ entscheidend fördern.

Beim Registerwertrecht verknüpft nun eine sog. Registrierungsvereinbarung Recht und Eintrag (Registrierung) in einem Wertrechtregister derart, dass das Recht nurmehr „über dieses Wertrechteregister geltend gemacht und auf andere übertragen werden kann“ (Art. 973d Abs. 1 Ziff. 2 OR).

Das Registerwertrecht ist mithin entgegen seiner Bezeichnung und der unglücklichen Legaldefinition ${ }^{9}$ gerade kein Recht. Stattdessen ist es, wie schon das Wertpapier, ${ }^{10}$ nur, aber immerhin, ein gesetzlich qualifiziertes Informationsmedium. ${ }^{11}$ An ihm können zwar Rechte erworben und sogar mit Wirkung gegenüber Dritten verteidigt und ausgeübt werden, ${ }^{12}$ wobei hier nun das Recht aus der Registrierung (Eintrag im Wertrechtregister) dem Recht an der Registrierung folgt. ${ }^{13}$ Dadurch wird aber die Registrierung, das Registerwertrecht, nicht selbst zum Recht, so dass eine Bezeichung als „Wertregistrierung“ treffender gewesen wäre. Wenigstens die englische Übersetzung als ledger-based security (wie auch die in der Praxis inzwischen geläufigere Bezeichnung register security) vermeidet die Bezugnahme auf das bloß repräsentierte Recht. ${ }^{14}$

Derartige terminologische Unstimmigkeiten sind solange unschädlich, als sie nicht zu unsachgerechten Schlüssen verleiten. Solche sind indes im noch jungen Schrifttum zum Registerwertrecht bereits erkennbar, ${ }^{15}$ so dass ihnen hier begegnet werden soll.

Inhaberpapier also der Urkundsbesitzer; bei Orderpapieren der dort oder in der lückenlosen Indossamentskette als berechtigt genannte Urkundsbesitzer). Die doppelte Legitimationsklausel (Inhaberklausel) verbietet es dem Schuldner, einen anderen Legitimationsnachweis zu verlangen. Meier-Hayoz/von der Crone, Wertpapierrecht (3. Aufl. 2018), $\$ 1$ Rn. $30 \mathrm{ff}$.

${ }^{8}$ Der Gläubiger eines unverurkundeten Rechts trägt die Beweislast für dessen Bestand (Art. 8 ZGB). Schon die Verurkundung in einem einfachen Schuldschein kehrt die Beweislast zwar um. Die Gläubigerstellung bleibt aber eine relative und die Verfügung über das Recht verlangt weiterhin einfache Schriftlichkeit (Art.165 Abs.1 OR), wobei gutgläubiger (unbelasteter) Rechtserwerb nur in den (praktisch kaum relevanten) engen Schranken von Art. 18 Abs. 2 und 164 Abs. 2 OR möglich ist (vgl. insb. Art. 167 und 169 OR). Erst die Verbriefung in einem Wertpapier führt zu Wirkungen erga omnes und erhöhter Umlauffähigkeit.

9 Vgl. Art. 973d pr OR: „Ein Registerwertrecht ist ein Recht, [...]“ (Hervorhebung hinzugefügt).

10 Vgl. Botschaft (Fn. 2), S. 243 und 259.

11 Vgl. schon Kubn, Digitale Aktiven im schweizerischen Privatrecht, in: Weber/Kuhn (Hrsg.), Entwicklungen im Schweizer Blockchain-Recht (2021), S. 51, Rn. 8. Der Bundesrat spricht von einem „qualifizierten Kleid“: Botschaft (Fn. 2), S. 277.

12 Vgl. IV. Wirkungen.

13 Es kann also, laut Bundesrat „das Recht im qualifizierten Kleid des Registerwertrechts übertragen werden“: Botschaft (Fn. 2), S. 277.

${ }_{14}$ Dabei sollte security (aus dem lateinischen securus bzw. sine cura bzw. ohne Sorge) nicht mit „Wertpapier" gleichgesetzt, sondern als (stoffunabhängiger, den Inhalt aber qualifiziert sichernder) Informationsträger verstanden werden. Nur so wird auch die (international ganz anerkannte) englische Übersetzung des Wertrechts als uncertificated security nicht zum Oxymoron (im Sinne eines „unverbrieften Wertpapiers“).

15 Vgl. IV.I.b und insb. Fn. 118. 
Der Name des Registerwertrechts lehnt sich leicht erkennbar an die „Wertrechte" an, welche seit 2010 in Art. 973c OR geregelt sind und nun zur besseren Abgrenzung in „einfache Wertrechte“ umbenannt wurden. Ihre bisherige Legaldefinition als „Rechte mit gleicher Funktion wie Wertpapiere“ wurde schon länger kritisiert ${ }^{16}$ und ist mit der Gesetzesrevision zu Recht entfallen. Denn die suggerierte funktionelle Äquivalenz von Wertrecht und Wertpapier beschränkt sich darauf, dass beide als Grundlage zur Schaffung der sogleich noch näher erläuterten Bucheffekten dienen (Art. 6 BEG). Die vom Wertpapier erzeugten privilegierten Rechtswirkungen (wie etwa dinglicher Schutz und Gutglaubenserwerb) vermag das schweizerische Wertrecht hingegen regelmässig nicht zu erzeugen, da ein dafür qualifiziertes Informationsmedium gerade fehlt: Sein Bestand ergibt sich zwar aus dem konstitutiven ${ }^{17}$ Eintrag in ein Wertrechtebuch (Art. 973c Abs. 3 OR). Letzteres ist indes nicht öffentlich, wird vom Schuldner selbst geführt und muss der Emission nachfolgende Verfügungen nicht dokumentieren. Entsprechend erlangt der Gläubiger eine bloss relative Rechtsposition, über die er nur durch schriftliche Abtretung verfügen kann (Art. 973c Abs. 4 i.V.m. 165 Abs. 1 OR), wenn er weder den Schuldner (mittels Vertragsübernahme) noch einen Dritten (durch Anweisung nach Art. $466 \mathrm{ff}$. OR) einbeziehen will. ${ }^{18}$

Damit unterscheidet sich das schweizerische Wertrecht - trotz gleichen Namens - fundamental von dem, was schon Opitz (auf den der Begriff massgeblich zurückgeht) als Wertrecht verstanden wissen wollte. Zwar ist auch bei ihm das „Wertrecht [...] im Gegensatz zum Wertpapier ohne körperliche Erscheinungsform“ und "Grundlage des Wertrechts das Schuldbuch". Indessen ist „nicht jede Schuldbuchforderung bereits Wertrecht“, sondern nur „die durch die Wertpapiersammelbank vermittelten Anteile an den auf den Namen der Wertpapiersammelbank eingetragenen Schuldbuchforderungen". ${ }^{19}$ Die so ein-

16 Vgl. nur Kuhn, Handkommentar zum Schweizer Privatrecht (3. Aufl. 2016), Art. 973c OR Rn. 1b; Kubn (Fn. 12), Rn. 19 und Fn. 42 m. w. H.

17 Vgl. auch Bundesrat, Botschaft zum Bucheffektengesetz sowie zum Haager Wertpapierübereinkommen, 15. November 2006, BBl. 2006, S. 9315, 9394. Zu Wertrechten ohne Wertrechtsbuch-Eintrag, welche dann nicht i.S.v. Art. 973c OR qualifizieren von der Crone/ Monsch/Meisser, Aktien-Token, Eine privatrechtliche Analyse der Möglichkeit des Gebrauchs von DLT-Systemen, GesKR 2019, 1, 4 und Fn. 34 m. w. H.

18 Eine Ausnahme bildeten nur die nach deutschem Vorbild gestützt auf das Bundesgesetz über das eidgenössische Schuldbuch vom 21. September 1939 (BBl. 1939 II 377) geschaffenen Schuldbuchforderungen gegenüber dem Bund: Art. 3 erklärte die Schuldbuchforderungen als „hinsichtlich Schuldverhältnis und Sicherheit den [verbrieften] Schuldverschreibungen der Eidgenossenschaft gleichgestellt“. Abgesehen davon bestand indes ein weiterer entscheidender Unterschied zu den Wertrechten nach Art. 973c OR: die Buchführung oblag der Nationalbank, die kraft ihres Mandates und ihrer institutionellen Fundierung den entsprechenden Ein- und Umbuchungen eine den Wertpapieren mindestens ebenbürtige Sicherheit verlieh. Vgl. auch Meier-Hayoz/von der Crone (Fn. 8), \$25 Rn. 29.

19 Opitz, Die Sammelverwahrung von Wertpapieren mit Gruppenauslosung (Bankwirt- 
gegrenzten Wertrechte rechnete Opitz den Sachen im Sinn des $\ 90$ BGB zu und verdinglichte sie gleichsam. ${ }^{20}$ Herrschend wurde indes die davon abweichende rechtstheoretische Begründung, nach der die Gleichbehandlung von Wertrecht und Wertpapier seit 1940 auf gesetzlicher Fiktion beruht. ${ }^{21}$ Sie erzielte zwar im Grundsatz dasselbe Ergebnis - die Anwendung des Sachenrechts auf die Wertrechte - verhinderte aber die von Opitz angestrebte Schaffung von Wertrechten jenseits der ausdrücklich positivierten Fälle. Entsprechend blieb die Emission von Wertrechten („Schuldbuchforderungen“ in der gesetzlichen Terminologie) beschränkt - derzeit auf juristische Personen des öffentlichen Rechts, so der Bund, die Länder und die Europäische Zentralbank.22

Wenn daher etwa die Rede ist von der „Funktionsäquivalenz“ des „Wertrechts" zum Wertpapier, ${ }^{23}$ so ist dies für das deutsche Recht zwar treffend, soweit die Wirkung der gesetzlich privilegierten Eintragung von Forderungen in ein Schuldbuch mit der Wirkung der Verbriefung von Forderungen verglichen wird. Dennoch bleibt der Terminus des Wertrechts - bei allem Respekt vor dem dogmatischen Gehalt, den ihm Lehre, Gesetzgebung und Rechtsprechung in Deutschland in den vergangenen 80 Jahren verliehen haben - ein misnomer, den zu rezipieren das schweizerische Recht ohne Verlust hätte lassen können.

Denn schon bislang erlaubte das deutsche Wertrecht keine dienlichen Schlüsse auf Rechtsnatur und Wirkung des einfachen Wertrechts schweizerischen Zuschnitts. Gleiches gilt für das neu geschaffene Registerwertrecht, das weit mehr als nur Anleihen zu repräsentieren vermag, auch von Privaten emittiert werden kann, keiner gesetzliche Einzelermächtigung bedarf und dessen Funktionen nicht pauschal dem Sachenrecht unterstehen, sondern über weite Strecken den Regeln der Wertpapiere öffentlichen Glaubens ${ }^{24}$ und konkret jenen über das Orderpapier ${ }^{25}$ nachgebildet sind.

Nach dem Gesagten hat der schweizerische Gesetzgeber mit dem Registerwertrecht also kein Recht geschaffen, sondern (wie schon im Falle des Wertpapiers) ein vom Privatrechtsverkehr ${ }^{26}$ entwickeltes Informationsmedium ${ }^{27}$

schaft, 1943, S. 35 ff., 55 ff., 77 ff.), in: ders. (Hrsg.), 50 depotrechtliche Abhandlungen (1954), S. 371,376 .

20 Vgl. nur etwa Opitz, Die gesetzliche Bestätigung der Wertrechtslehre (Bank-Archiv, 1942, S. 199 ff.), in: ders. (Hrsg.), 50 depotrechtliche Abhandlungen (1954), S. 455, 460.

${ }^{21}$ BGH, 1.2.1952, BGHZ 5, 27-35, 31.

22 Vgl. etwa Schwarz, Globaler Effektenhandel (2016), S. 51 ff., 54f.m.w.H.; Art. 2 II des Gesetzes zu dem Abkommen v. 18.9.1998 zwischen der Regierung der Bundesrepublik Deutschland und der Europäischen Zentralbank über den Sitz der Europäischen Zentralbank v. 19.12.1998 (BGBl. 1998 II, S. 2995).

${ }^{23}$ So Schwarz (Fn. 23), S. 52.

${ }^{24}$ Vgl. schon Kubn (Fn. 12), Rn. 16.

25 Botschaft (Fn. 2), S. 285.

26 Botschaft (Fn. 2), S. 243.

27 Die Botschaft (Fn. 2), S. 277 spricht statt von „Informationsträger“ von einem „qualifizierten Kleid“. Vgl. auch S. 258, 275, 287. 
mit spezifischen Privilegien betreffend Transport, Legitimation und Verkehrsschutz ausgestattet und so die Umlauffähigkeit der mit ihm dargestellten Rechte wesentlich erhöht. ${ }^{28}$ Die Voraussetzungen dazu werden später noch näher erläutert. ${ }^{29}$

Der Vorgang als solcher ist keineswegs neu, hat doch der Gesetzgeber bereits mit dem 2010 in Kraft getretenen Bucheffektengeset $z^{30}$ die Möglichkeit geschaffen, ausgewählte Rechte einem umlauffähigeren Medium, der Bucheffekte, zuzuführen. ${ }^{31}$ Sie entsteht, wenn fungible Forderungs- und Mitgliedschaftsrechte i.S. v. Art. 3 Abs. 1 BEG bei einer Verwahrungsstelle i.S. v. Art. 4 BEG auf einem Effektenkonto i.S.v. Art. 2 Abs. 1 BEG eingebucht werden und zugleich deren bisherige Form inaktiviert wird (Art. 6 Abs. 1 BEG) ${ }^{32}$ Verfügungen über das Recht erfolgen sodann nurmehr mittels Verfügung über die Bucheffekte „nach den Vorschriften dieses Gesetzes“ (Art. 3 Abs. 1 Bst. b BEG). Als Regel sieht es die abstrakte, weisungsbasierte Kontobuchung vor (Art. 24 Abs. 1 BEG); bei Sicherung und Nutznießung auch die schlichte (kausale) Einigung zwischen Kontoinhaber und Verwahrungsstelle (Art. 25 f. BEG). Der entgeltliche Erwerb genießt Gutglaubensschutz (Art.29 BEG). Zudem entfaltet die Bucheffekte Wirkung erga omnes (Art. 3 Abs. 2 BEG) und erlaubt namentlich die Absonderung in der Insolvenz der Verwahrungsstelle (Art. 17-19 BEG).

Die entsprechende Privilegierung der Bucheffekte gründet im geschlossenen Kreis der zugelassenen Verwahrungsstellen, welche als prudenziell beaufsichtigte Finanzintermediäre einem engmaschigen Netz von regulatorischen Vorschriften unterstehen. Anders als die Wertrechtsbücher einfacher privater Schuldner bieten ihre Buchungen (die Bucheffekten), qualifizierte Gewähr für Richtigkeit - oder zumindest Gewähr für die Solvenz der Verwahrungsstelle, um die Richtigkeit zulasten der eigenen Mittel (wieder-)herstellen zu können. ${ }^{33}$

Zusammenfassend ergibt sich, dass das schweizerische Recht mit dem Registerwertrecht nach dem Wertpapier, der Bucheffekte, dem hier nicht näher

28 Sinngemäß auch Botschaft (Fn. 2), S. 276 (Hervorhebung hinzugefügt): „Mit Artikel 973d E-OR wird neu die elektronische Registrierung von Rechten mit Wertpapierwirkungen ermöglicht."

29 Vgl. III. Voraussetzungen.

30 Bundesgesetz über Bucheffekten (BEG), 3. Oktober 2008, SR 957.1.

31 Ausführlich Zellweger-Gutknecht, Vermögenswerte im Finanzmarktrecht - Das Ende aller dinglichen Prinzipien?, in: Domej/Dörr/Hoffmann-Nowotny/Vasella/Zelger (Hrsg.), Einheit des Privatrechts, komplexe Welt (2009), S. 87.

32 Wertpapiere werden bei einer Verwahrungsstelle zur Sammelverwahrung hinterlegt; Globalurkunden bei einer Verwahrungsstelle hinterlegt, einfache Wertrechte im Hauptregister einer Verwahrungsstelle eingetragen und Registerwertrechte auf eine Verwahrungsstelle übertragen. Die Inaktivierung verhindert, dass mehrere Informationsträger parallel zirkulieren und das in ihnen abgebildete Recht (durch Gutglaubenserwerb) zu Lasten der Emittentin multiplizieren. Zellweger-Gutknecht (Fn. 32), S. 94.

${ }^{33}$ Eingehend Zellweger-Gutknecht (Fn. 32), S. 95 f. 
thematisierten Register-Schuldbrief ${ }^{34}$ und Einträgen in diversen weiteren Registern $^{35}$ ein weiteres qualifiziertes Informationsmedium hervorgebracht hat, das ausgewählte Rechte abbilden, nach eigenen, privilegierten Regeln mobilisieren und mit Drittwirkung versehen kann. Demgegenüber war und ist das schweizerische Wertrecht (mit Ausnahme der abgeschafften eidgenössischen Schuldbuchforderungen) gerade kein derart qualifiziertes Informationsmedium. Entsprechend gelten trotz Eintrags im Wertrechtebuch einzig die für das betreffende Recht einschlägigen Regeln. Wird ein Recht hingegen in ein qualifiziertes Informationsmedium gekleidet oder ein Informationsmedium in ein anderes umgewandelt, so folgt das Recht fortan dessen Regeln. Im verbleibenden Teil des Beitrages werden die für das Registerwertrecht wesentlichen Regeln betreffend Voraussetzungen und Wirkungen dargestellt.

\section{Voraussetzungen}

Damit ein elektronischer Eintrag als Registerwertrecht qualifiziert, werden vier Tatbestandselemente vorausgesetzt: erstens muss ein Recht vorliegen und zweitens ein Wertrechteregister bestehen, die beide den gesetzlichen Anforderungen zu genügen haben. Sodann sind, drittens, Recht und Register mittels einer Registrierungsvereinbarung rechtlich so zu verknüpfen, dass über das Recht nur noch auf dem Register verfügt werden kann. Viertens muss schließlich die Verknüpfung mittels Registrierung des Rechts auf dem Register auch faktisch vollzogen werden.

\section{Registerwertrechtsfähige Rechte}

Laut Gesetz kommen als Inhalt von Registerwertrechten nur „Rechte“ in Frage (Art. 973d Abs.1 OR). Weitere Präzisierungen sind nicht positiviert, sondern lediglich den Gesetzesmaterialien zu entnehmen. So brauchen betreffende Rechte weder zum massenweisen Handel geeignet noch überhaupt vertretbar zu sein. ${ }^{36}$ Sie können zudem entweder bereits vorbestehen oder mit der Registrierung neu geschaffen werden. ${ }^{37}$ Weiter muss es sich um Rechte handeln, die zumindest im Grundsatz übertragbar sein müssen, ${ }^{38}$ wohingegen eine rechts-

\footnotetext{
${ }^{34}$ Er ist seit dem 1. Januar 2012 im Sachenrecht in den Art. 843 und 860-865 ZGB verankert.

${ }^{35}$ Grundbuchregister, Viehregister, Luftfahrzeugbuch, Schiffsregister, Marken-, Patentund Designregister etc.

${ }^{36}$ Botschaft (Fn.2), S. 277; Kramer/Oser/Meier, Tokenisierung von Finanzinstrumenten de lege ferenda, in: Jusletter (6. Mai 2019), Rz.21.

37 Botschaft (Fn. 2), S. 276.

38 Botschaft (Fn. 2), S. 243; zu eng hingegen Kramer et al. (Fn. 38), Rz. 21 m. w. H. wonach die Übertragbarkeit in Anlehnung an Art.164 OR auch nicht durch Vereinbarung oder die Natur des Rechts ausgeschlossen sein darf.
} 
geschäftliche oder statutarische Beschränkung wie etwa ein pactum de non cedendo, ein Aktionärsbindungsvertrag oder eine Vinkulierung die Registerwertrechtsfähigkeit nicht beeinträchtigt. Soweit sich solche Beschränkungen nicht aus dem Registerwertrecht oder damit verknüpften Begleitdokumenten ergeben, greifen sie indes nur, wenn der Erwerber beim Erwerb bösgläubig oder grobfahrlässig handelte (Art. 973e Abs. 3 und 4 OR). ${ }^{39}$

Weiter sollen „[a]lle Rechte, die [...] in Wertpapieren verbrieft werden können, auch als Registerwertrechte ausgestaltet werden" können. ${ }^{40}$ Das ist indes zu weit gefasst: Da die Wirkungen des Registerwertrechts den Wertpapieren öffentlichen Glaubens nachempfunden werden sollen, reicht folglich die Verbriefbarkeit in Wertpapiere ohne öffentlichen Glauben nicht aus. ${ }^{41}$

Registerwertrechtsfähig sind grundsätzlich alle Arten von obligatorischen Ansprüchen. ${ }^{42}$ Hingegen existieren im Bereich der Mitgliedschafts- und Sachenrechte leges speciales, welche eine Registrierung erschweren oder verhindern.

So ist namentlich Grundeigentum als solches nicht registerrechtsfähig, da mit dem Grundbucheintrag bereits ein qualifiziertes Informationsmedium besteht, das exklusive Geltung beansprucht (Art.656 Abs. 1 ZGB). ${ }^{43}$ Mit dem numerus clausus der dinglichen Rechte vereinbar sind nur gerade die Anleihenstitel mit Grundpfandrecht (Art. $875 \mathrm{ZGB})^{44}$ und - theoretisch - der Schuldbrief (Art. $842 \mathrm{ff}$. ZGB). Sowohl der Register- als auch der Papierschuldbrief verlangen für die Begründung der Schuldbriefforderung indes wiederum einen Grundbucheintrag, der Registerschuldbrief zudem auch bei jeder Übertragung (Art. 857 Abs. 1, 858 Abs. 1 und 860 Abs. 1 ZGB). Registerwertrechte könnten daher höchstens bei gestuftem Besitz an Papierschuldbriefen oder bei der Zwischenschaltung eines Nominees beim Registerschuldbrief zum Einsatz kommen; in diesen Fällen vermögen sie den mittelbaren Besitzer zur Besitzanweisung (Art. 924 Abs. 1 Var. 1 ZGB) und dort den wirtschaftlich Berechtigten zur Anweisung (Art. 466ff. OR) zu legitimieren. ${ }^{45}$

Auch Eigentum an Fahrnis ist als solches nicht registerwertrechtsfähig, weil auch hier das Gesetz mit dem Besitz bereits ein qualifiziertes „Informations-

39 Vgl. auch Kubn (Fn. 12), Rn. 77.

40 Botschaft (Fn. 2), S. 276.

${ }^{41}$ Botschaft (Fn. 2), S. 284. Daher sind etwa GmbH-Anteile nicht registerwertrechtsfähig, da sie lediglich in Form von Beweisurkunden oder Namenpapieren begeben werden dürfen, vgl. bei Fn. 49 .

42 Botschaft (Fn. 2), S. 276.

${ }^{43} \mathrm{Zu}$ (indirekten) Möglichkeiten der Registrierung von Beteiligungen an Immobiliengesellschaften, -fonds und -trusts Kubn (Fn. 12), Rn. 91.

${ }^{44}$ Inhaber- oder Namensobligation mit Grundpfandverschreibung oder mit Schuldbrief (auch: Hypothekarobligation). So wird etwa bei der Obligation mit Grundpfandverschreibung eine verbriefte Forderung mit einem unverbrieften Grundpfandrecht gesichert: BGer, 5A_331/2018, 21.12.2018 E 6.2; 135 III 378 E. 2.3.

${ }^{45}$ Zum Nominee-Modell Kubn (Fn. 12), Rn. 93. 
medium“ zwingend vorschreibt. Gangbar bliebe aber wiederum die registerbasierte Besitzanweisung im gestuften Besitz ${ }^{46}$ und zudem der Einsatz eines Registerwertrechts als „Mittel“, das dem Empfänger i.S.v. Art. 922 Abs. 1 Var. 2 ZGB Gewalt über eine Sache verschafft ${ }^{47}$ sowie die neu erlaubte Registrierung von Warenpapieren auf einem Wertrechtsregister (Art.1153a OR).

Etwas weniger limitiert sind die Verhältnisse im Gesellschaftsrecht, das freilich ebenfalls durch einen numerus clausus beherrscht wird. So sind der Verbriefung in einem Wertpapier öffentlichen Glaubens immerhin Aktien und Partizipationsscheine von Aktien- und Kommanditaktiengesellschaften zugänglich, sofern die Statuten es zulassen (Art. 622, 656a Abs. 2 und 764 Abs. 2 OR), nicht aber die Anteile an einer GmbH oder einer Genossenschaft (Art. 784 Abs. 1 und 849 Abs. 1 ZGB). ${ }^{48}$ Verbriefungsunabhängige Übertragungsbeschränkungen wie namentlich die Vinkulierung von nicht börsenkotierten Aktien (Art. 685 a ff. OR) stehen einer Registrierung in einem Wertrechteregister hingegen nicht entgegen. ${ }^{49}$ Allerdings muss hier die Gesellschaft dafür sorgen, dass die Übertragung auf dem Register vor der Zustimmung der Gesellschaft technisch gar nicht erst möglich ist. ${ }^{50}$

Explizit und pauschal von der Registrierung in einem Wertrechteregister ausschliessen wollte der Bundesrat hingegen die Registrierung von ZahlungsToken bzw. die synonym erwähnten reinen Kryptowäbrungen oder „kryptobasierte Zahlungsmittel, welche von keinem Emittenten herausgegeben werden“ mit der Begründung, dass diese nach wohl h. L. rein faktische, immaterielle Vermögenswerte seien, für deren Übertragbarkeit das Zivilrecht keine Hindernisse aufstelle und mithin kein gesetzlicher Handlungsbedarf bestehe. ${ }^{51}$ Der Begriff der Zahlungs-Token geht zurück auf die in der Aufsichtspraxis der schweize-

${ }^{46}$ Zur Variante Miteigentum i. S. v. Art. 646ff. ZGB (etwa an Kunstwerken) zu begründen und über diese Individualrechte an einer rechnerischen Quote mittels Besitzanweisung an einen Lagerhalter (Art. 482 Abs. 1 OR) zu verfügen Rüfenacht, Die Tokenisierung von Anteilen an Kunstwerken: Zur Eignung von Miteigentum, Registerwertrechten und kollektiven Kapitalanlagen, Magister, Editions Weblaw (2021), S. 20; ders., Tokenisierte Anteile an Kunstfonds - Zur Eignung von Registerwertrechten und Kryptowährungen in kollektiven Kapitalanlagen, Jusletter vom 3. Mai 2021.

${ }^{47}$ Bundesrat, Rechtliche Grundlagen für Distributed Ledger-Technologie und Blockchain in der Schweiz, Eine Auslegeordnung mit Fokus auf dem Finanzsektor, 14.12.2018, S. 65.

48 Weiterführend MacCabe (Fn. 6), S. $147 \mathrm{ff}$.; Morscher/Staub, Tokenisierung von Effekten für Private Equity und Private Debt, in: Gericke/Europa Institut der Universität Zürich (Hrsg.), Venture Capital Reinvented - Markt, Recht, Steuern (2021), S. 193, https://doi. org/10.36862/eiz-357 (alle Hyperlinks in diesem Beitrag wurden zuletzt am 20.05.2021 abgerufen); von der Crone/Baumgartner, Digitalisierung des Aktienrechts - Die Ausgabe von Aktien als Registerwertrechte, SZW 2020, 351, 360; noch zur Rechtslage vor dem Inkrafttreten der Gesetzesrevision von der Crone/Monsch/Meisser (Fn. 18), 1. Meier-Hayoz/von der Crone (Fn. 8), N $13 \mathrm{ff}$.

49 Desgleichen verhindert sie auch eine Abbildung in Bucheffekten nicht: Art. 24 Abs. 4 BEG.

${ }^{50}$ Botschaft (Fn. 2), S. 274; Morscher/Staub (Fn. 49), S. 212.

51 Zit. Botschaft (Fn. 2), S. 277; vgl. auch S. 242 und 258 f. 
rischen Finanzmarktaufsichtsbehörde FINMA entwickelte Kategorisierung. ${ }^{52}$ Ihr zufolge fungieren Zahlungs-Token als Tauschmittel, Wertspeicher und/ oder Recheneinheit, wobei erstere Funktion meist dominiert und ein intrinsischer Wert fehlt. Nutzungs-Token vermitteln Zugang zu digitalen Diensten oder Waren. Anlage-Token repräsentieren schließlich Vermögensanlagen etwa in Form von schuldrechtlichen Ansprüchen und/oder gesellschaftsrechtlichen Mitgliedschaftsrechten oder Derivate solcher Rechte. Schließlich existieren hybride Typen.

Der beabsichtigte Ausschluss von Kryptowährungen von Art. 973dff. OR ist in mehrfacher Hinsicht zu kritisieren bzw. zu relativieren. Erstens führt die Kategorisierung in der Praxis zu erheblichen Abgrenzungsschwierigkeiten, nur schon weil an sich als Zahlungsmittel intendierte Werte vom Publikum aus (spekulativen) Anlagemotiven erworben und gehalten werden. ${ }^{53}$

Zweitens bilden Kryptowährungen nach hier vertretener Auffassung per se Rechtspositionenen ab. Im Falle von konvertiblen Stablecoins ${ }^{54}$ besteht eine dieser Rechtspositionen in einem klassischen Forderungsrecht. Doch selbst bei fehlender Konvertibilität sind Kryptowährungen jedenfalls mit staatlichem FiatGeld (Banknoten und Sichtguthaben) vergleichbar. ${ }^{55}$ Die wohl h. L. qualifiziert letzteres (noch) als Verbindlichkeit der Zentralbank; richtigerweise handelt es sich freilich um eine den eigenen Mitteln näherstehende Form von Passiven der Zentralbank. ${ }^{56}$ So oder so handelt es sich aber jedenfalls nicht um einen rein faktischen Vermögenswert. Hinzukommt, dass der Emittent mit der Ausgabe von Kryptowährung naturgemäß auch die Verpflichtung eingeht, deren Verwendung zur Zahlungsabwicklung nach verbindlichen Regeln zu gewährleisten oder gar das ihr unterliegende Zahlungssystem zu betreiben. Schon daraus

\footnotetext{
52 Vgl. zum Folgenden FINMA, Wegleitung für Unterstellungsanfragen betreffend Initial Coin Offerings (ICOs) (16. Februar 2018).

${ }^{53} \mathrm{Vgl}$. Fusswinkel/Kreiterling, Blockchain-Technologie-Gedanken zur Regulierung, in: Bafin Perspektiven 1 (2018), S. 48, 57.

${ }^{54}$ Vgl. dazu etwa Vgl. Zellweger-Gutknecht/Weber, Private Zahlungsmittel und Zahlungssysteme, Jusletter (11. Januar 2021), Rn. 27 ff. m. w. H.

55 Vgl. schon Kubn (Fn. 12), Rn. 96 und Weber, Neue Blockchain-Gesetzgebung in der Schweiz, Recht Digital (RDi) 4 (2021), 186 Rn. 8. Vgl. auch die Kritik bei Swiss Blockchain Federation, Zirkular 2021/01 Registerwertrechte (1. Februar 2021), http://blockchainfederation.ch/wp-content/uploads/2021/01/Circular-2021_01-Registerwertrechte.pdf (18.05.2021) S. 11.

56 Vgl. dazu Zellweger-Gutknecht, Developing the Right Regulatory Regime for Cryptocurrencies and other Value Data, in: Fox/Green (Hrsg.), Cryptocurrencies in Public and Private Law (2019), Rn. 4.06ff and 4.12ff. (Banknoten), 4.22 und 4.27ff. (Sichtguthaben), 4.44 (Fazit); und schon dies, ,Negativzins' und Bilanzsituation der SNB aus monetärrechtlicher Sicht, Jusletter (9. Februar 2015); Barker/Bholat/Ryland, Central Bank Balance Sheets: Past, Present and Future, in: Bank Underground (blog for Bank of England staff) (3. Juli 2017). Jünst nun auch Kumbof/Allen/Bateman/Lastra/Gleeson/Omarova, Central Bank Money: Liability, Asset, or Equity of the Nation? Cornell Legal Studies Research Paper (17. November 2020), S. 20-46.
} 
wird deutlich, dass Zahlungs-Token stets insofern auch als Nutzungs-Token qualifizieren, als sie den Zugang zu einem Zahlungssystem bzw. zur digitalen Nutzung der Token als Zahlungsinstrumente vermitteln. Dadurch beinhalten aber auch Kryptowährungen nicht nur eine nach Art. 973d OR vorausgesetzte Rechtsposition, sondern sind als Nutzungs-Token schon nach der ausdrücklichen Auffassung des Bundesrates auch registerwertrechtsfähig. ${ }^{57}$

Drittens ist die Botschaft des Bundesrates selbst keine Rechtsquelle und damit für Gerichte bei der Auslegung des Gesetzes im Streitfall bloße Rechtserkenntnisquelle. Zudem wurde der Ausschluss von Kryptowährungen im Parlament nicht diskutiert, so dass „der Gesetzgeber“ die Frage jedenfalls nicht entschieden hat.

Viertens schließlich zeigt ein Blick über die Landesgrenze nach Liechtenstein, dass die Zulassung von Kryptowährungen zur Registrierung auf einem Wertrechteregister kaum zu unerwünschten Folgen führen dürfte: Dort steht mit dem Gesetz über Token und VT-Dienstleister (TVTG ${ }^{58}$ seit Januar 2020 ein zivil- und aufsichtsrechtlicher Rahmen in Kraft, der selbst sog. „leere“ Token erfasst, welche keine Rechtsposition vermitteln. Davon ausgenommen sind freilich Token ohne identifizierbaren Emittenten, da sie die Registrierungspflicht nach Art. 12 TVTG naturgemäß nicht erfüllen.

Im Ergebnis ist festzuhalten, dass prinzipiell jede verbriefbare Rechtsposition auch registerwertrechtsfähig ist - darin eingeschlossen Kryptowährungen, da diese den Nutzern jedenfalls den schuldrechtlichen Anspruch gegenüber dem Emittenten vermitteln, die Zahlungsabwicklung technisch zu gewährleisten und sie insofern auch als Nutzungs-Token qualifizieren. Nur bei rein dezentralen Kryptowährungen ohne identifizierbaren Emittenten dürfte eine Subsumtion unter die Art. $973 \mathrm{~d}$ ff. OR scheitern - weil sonst die an den „Schuldner“ adressierten Pflichten und Haftungsbestimmungen ins Leere liefen.

\section{Wertrechteregister}

$\mathrm{Da}$ Wertrechteregister und Registereintrag vergleichbare Wirkungen entfalten sollen wie traditionelle Wertpapiere öffentlichen Glaubens, listet Art.973d Abs. 2 OR vier funktionale Voraussetzungen auf, welche das Register aufzuweisen hat, wenn die auf ihm getätigten Einträge als Registerwertrechte qualifizieren sollen. Hingegen hat der Gesetzgeber die technische Umsetzung bewusst der Praxis überlassen, nachdem entsprechende Vorschriften bzw. Ver-

${ }^{57}$ Botschaft (Fn. 2), S. 277. Vgl. schon Bundesrat, Rechtliche Grundlagen für Distributed Ledger-Technologie und Blockchain in der Schweiz: Eine Auslegeordnung mit Fokus auf dem Finanzsektor (14. Dezember 2018), S. 67.

58 Mit „VT“ werden vertrauenswürdige Technologien bezeichnet; Regierung, Bericht und Antrag an den Landtag betreffend die Schaffung eines Gesetzes über Token und VTDienstleister (Token- und VT-Dienstleister-Gesetz; TVTG) und die Abänderung weiterer Gesetze (7. Mai 2019), BuA 054/2019, LNR 2019-51. 
ordnungsvorbehalte im Vernehmlassungsverfahren breit kritisiert wurden. ${ }^{59}$ Inzwischen sind erste Branchenstandards geschaffen worden, welche den Beteiligten zusätzliche Orientierungshilfe bieten. ${ }^{60}$

Zunächst muss das Wertrechteregister mittels technischer Verfahren dem Gläubiger Verfügungsmacht über die abgebildeten Rechte vermitteln und zugleich den Schuldner von ebendieser Verfügungsmacht ausschließen (Art. 973d Abs. 2 Ziff. 1 OR). ${ }^{61}$ Die Gläubiger sollen also in die Lage versetzt werden, „die Übertragung von Registerwertrechten [...] grundsätzlich ohne Zutun einer vertrauenswürdigen zentralen Instanz, welche das Register alleine verwaltet, [auszulösen] und nach den Regeln des Wertrechteregister [durchzuführen]“, was ein Mindestmaß an Dezentralität voraussetzt. ${ }^{62}$ Die Verfügungsmacht ist zu verstehen als technisch gewährleistete Rivalität ${ }^{63}$ und Exklusivität ${ }^{64}$ und entspricht sinngemäß der sachenrechtlichen faktischen Beherrschbarkeit durch Besitz. Sie muss allerdings nur dem Grundsatz nach eingeräumt sein und nicht zwingend jederzeit bestehen.

Ungeklärt ist in diesem Zusammenhang bislang die Frage, inwieweit einerseits der Schuldner von der Verfügungsmacht ausgeschlossen und andererseits doch sichergestellt werden soll, dass im gesetzlich zulässigen Rahmen (namentlich nach einer Kraftloserklärung oder einer Zwangsvollstreckung in das Vermögen des Gläubigers) auch ohne Mitwirkung des Gläubigers über ein Registerwertrecht disponiert werden kann. ${ }^{65}$

Nach Ziffer 2 muss das Wertrechteregister durch angemessene technische und organisatorische Massnahmen Integrität gewährleisten. Das umfasst namentlich eine angemessene Authentifizierung, Autorisierung, Resilienz und Konfliktresolution und soll unbefugte Veränderung der Daten und Operationen verhindern. Das Gesetz verweist hier im Sinne eines minimalen Sicherheitsstandards beispielhaft auf die dezentrale Verwaltung. Letztlich muss das

59 Botschaft (Fn. 2), S. 279.

$60 \mathrm{SBF}$ (Fn. 56)

61 Vgl. zur Parallele zum Wertpapier Kubn/Stengel/Meisser/Weber, Wertrechte als Rechtsrahmen für die Token-Wirtschaft, Jusletter IT (23. Mai 2019), Rn. $15 \mathrm{ff.}$

62 Botschaft (Fn. 2), S. 278.

63 Vgl. schon Meisser/Meisser/Kogens, Verfügungsmacht und Verfügungsrecht an Bitcoins im Konkurs, Jusletter IT (24. Mai 2018), Rn. 8 und 15: Bei rivalisierenden Vermögensgüter beeinträchtigt oder verunmöglicht die Nutzung einer Person die Nutzung desselben Gutes durch weitere Personen. Bei Kryptowerten beruht die Rivalität namentlich darauf, dass double-spending technisch verhindert wird.

64 Dazu Zellweger-Gutknecht (Fn. 58), Rn. 4.81 m. w. H.: Exklusiv sind Güter, bei denen es möglich oder jedenfalls wirtschaftlich sinnvoll ist, Nicht-Erwerber vom Konsum auszuschließen. Erfüllt ein Vermögenswert beide Eigenschaften, ist er mithin rival und exklusiv zugleich, kann ein Rechtssubjekt an ihm Eigentum oder eine vergleichbar weitreichende Rechtszuständigkeit erlangen.

65 Vgl. SBF (Fn. 56) Zirkular 2021/01, S. 4. 
Register in der Lage sein, die Rechtslage korrekt und dauerhaft bzw. reproduzierbar abzubilden. ${ }^{66}$

Ziffer 3 verlangt sodann, dass das Wertrechteregister die ausgewählte Essentialien im Code selbst oder in mit dem Code verknüpften ${ }^{67}$ Begleitdaten (etwa Gesellschaftsstatuten, Emissionsbedingungen, White Paper) permanent und zugänglich festhält. Zu den erwähnten Essentialien gehören der Inhalt der im Register abgebildeten Rechte (so namentlich die Person des Schuldners, die Art des Rechts, sein Nominalwert und seine Stückelung sowie die Gesamtkapitalisierung und der Verfall), ${ }^{68}$ die Funktionsweise des Registers in Bezug auf Verfügungsmacht und Integrität, sowie die sogleich noch näher erläuterte Registrierungsvereinbarung. Im Kern müssen jene Basisinformationen publik gemacht werden, die notwendig sind, um ein bestimmtes Registerwertrecht bzw. das darin abgebildete Recht eindeutig bestimmen zu können. ${ }^{69}$

Ziffer 4 verlangt schließlich sog. relative Publizität in dem Sinne, dass Gläubiger die sie betreffenden Informationen und Einträge konsultieren und die Integrität des sie betreffenden Registerinhaltes selbständig überprüfen können allerdings nur in der Regel und nicht zwingend jederzeit. ${ }^{70}$ Damit nähert das Gesetz die Position des Inhabers eines Registerwertrechts an jene des Inhabers eines Wertpapiers an, der ebenfalls ohne Mitwirkung des Schuldners prüfen kann, ${ }^{71}$ ob es verändert oder gefälscht wurde. Idealerweise legt ein Register zu diesem Zweck seinen Quellcode offen. ${ }^{72}$

\section{Registrierungsvereinbarung}

Mit der Registrierungsvereinbarung nach Art.973d Abs. 1 pr OR wird ein Recht so mit einem Wertrechteregister verquickt, dass es fortan nur noch über dieses Register - durch Verfügung über das Registerwertrecht - geltend gemacht und übertragen werden kann. Zu diesem Zweck vereinbaren die Parteien (der Schuldner und der erste Nehmer, d.h. jene Person, welche das Registerwertrecht als erste rechtsgeschäftlich übernimmt), dass das Recht durch ein im Wertrechteregister eingetragenes Registerwertrecht abgebildet (registriert) wird. Die Registrierungsvereinbarung enthält damit automatisch stets auch eine dem wertpapierrechtlichen Begebungsvertrag ${ }^{73}$ entsprechende Begebungs-

66 Botschaft (Fn. 2), S. 279.

67 Botschaft (Fn. 2), S. 283; SBF (Fn. 61) Zirkular 2021/01, S. 6.

68 Botschaft (Fn. 2), S. 282; SBF (Fn. 61) Zirkular 2021/01, S. 6.

$69 \mathrm{Kubn}$ (Fn. 12) Rn. 63 ff. zu den Gründen, weshalb für Registerwertrechte keine weitergehende Informationsleistung verlangt werden kann als für Wertpapiere.

70 Weber (Fn. 56), Rn. 15.

71 Botschaft (Fn. 2), S. 283; SBF (Fn. 62) Zirkular 2021/01, S. 7.

$72 \mathrm{Kuhn}$ (Fn. 12), Rn. 68 m. w. H.

73 Vgl. BVGer, 11.5.2009, A-6020/2007, E. 4.2. 
abrede. ${ }^{74}$ Spätere Erwerber treten der Vereinbarung sodann durch Erwerb des Registerwertrechtes bei. ${ }^{75}$

Die Vereinbarung muss weiter vorsehen, dass fortan der Schuldner nur an den durch das Wertrechteregister ausgewiesenen Gläubiger sowie gegen entsprechende Anpassung des Registers zu leisten berechtigt und verpflichtet ist (Präsentationswirkung) und durch eine bei Verfall an diesen erfolgte Leistung befreit wird, auch wenn der ausgewiesene nicht der tatsächliche Gläubiger ist, sofern dem Schuldner weder Arglist noch grobe Fahrlässigkeit zur Last fällt (Legitimationswirkung). Funktionell entspricht die Registrierungsvereinbarung damit sinngemäß einer qualifizierten Wertpapierklausel, enthält also sowohl eine doppelseitige Präsentationsklausel als auch eine doppelseitige Legitimationsklausel, wobei statt der entsprechenden Formel auch ein Verweis auf die einschlägigen Art. 973e Abs. 1 und 2 OR genügen muss. ${ }^{76}$

Unverzichtbar ist sodann die Regelung des Verfügungsmodus. Denn das Gesetz legt diesen nicht selbst fest, sondern verweist in Art. 973f Abs. 1 OR schlicht auf die „Regeln der Registrierungsvereinbarung“. Zu diesem Zweck wird die Vereinbarung das unterliegende Protokoll bezeichnen und umso detaillierter umschreiben, je weniger bekannt und verbreitet es ist. ${ }^{77}$ Dazu gehören etwa der Konsensmechanismus und die Weise, wie Transaktionen in das System eingebracht, validiert und priorisiert werden. Weiter ist zu klären, wann eine Transaktion rechtlich unwiderruflich und wann sie final, ${ }^{78} \mathrm{~d}$.h. wirksam werden soll (z. B. nachdem die Validierung abgeschlossen und Konflikte zwischen sich widersprechenden Transaktionen mit hinreichender Klarheit aufgelöst sind). ${ }^{79}$ Hingegen ist es nach hier vertretener Auffassung nicht erforderlich, bei an Ordre gestellten Registerwertrechten eine Bestimmung aufzunehmen, wonach die Übertragung nach den Regeln des Systems als Indossament qualifiziere. ${ }^{80}$

Repräsentieren Registerwertrechte eine gesellschaftsrechtliche Rechtsposition (Aktien, Partizipationsrechte, Genussscheine etc.), ersetzt eine entsprechende statutarische Grundlage die Registrierungsvereinbarung (Art.622 Abs. 1 OR). Zudem kann der Verwaltungsrat statutarisch ermächtigt werden, die Einzelheiten der Registrierung in einem Reglement festzulegen. ${ }^{81}$

\footnotetext{
${ }^{74} \mathrm{Kubn}$ (Fn. 12), Rn. $106 \mathrm{f}$.

${ }^{75} \mathrm{Kuhn}$ (Fn. 12), Rn. 101.

76 Ungenau hingegen Botschaft (Fn. 2), S. 276, welche nur eine einfache Wertpapierklausel verlangt und damit übergeht, dass Registerwertrechte den Wertpapieren öffentlichen Glaubens nachgebildet sind. Wie hier schon Kubn (Fn. 12), Rn. 99.

77 SBF (Fn. 61), Zirkular 2021/01, S. 10.

${ }_{78}$ Zur in Art. 973f Abs. 2 OR geregelten Finalität in der Insolvenz vgl. IV.1.b.

79 Vgl. zur Unwiderruflichkeit Art.9 OR; zur Finalität Art.10 OR: Rückdatierung auf den Zeitpunkt, wo die fragliche Erklärung „zur Absendung abgegeben“ wurde, d.h. die Rechtssphäre des Erklärenden verlassen hat.

${ }^{80}$ Vgl. eingehend unten IV.1.b; a. M. Kubn (Fn. 12), Rn. 103.

${ }^{81}$ SBF (Fn. 61) Zirkular 2021/01, S. 9.
} 
Das Gesetz verlangt für die Registrierungsvereinbarung zwar keine bestimmte Form, setzt mit Art.973d Abs. 2 Ziff. 3 und 4 OR aber immerhin voraus, dass die Codierung im Register oder die Verknüpfung mit dem Code umsetzbar und die selbstständige Einsichtnahme durch die Gläubiger gewährleistet sein müssen, was nach heutigem Stand der Technik einen Nachweis in Text zumindest ermöglichen muss. Schließlich darf die Vereinbarung auch als AGB ausgestaltet sein. ${ }^{82}$

Idealerweise sollte die Registrierungsvereinbarung schließlich jene Aspekte regeln, die der Gesetzgeber der Privatautonomie überlassen hat. Dazu zählen etwa: die Rechtswahl (Art. 145a IPRG), ${ }^{83}$ der Wechsel des Wertrechtregisters und die Konditionen für den Wechsel des Informationsmediums (z. B. Umwandlung in eine Bucheffekte), ${ }^{84}$ die nicht-rechtsgeschäftliche Übertragung etwa bei Universalsukzession, ${ }^{85}$ das Vinkulierungsregime ${ }^{86}$ sowie die (nicht Art. $973 \mathrm{~d}$ ff. OR unterfallenden) Folgen von off-chain Transaktionen ${ }^{87}$ inklusive Gutglaubenserwerb, ${ }^{88}$ eine Vereinfachung der Kraftloserklärung (Art. 973h Abs. 2 OR ${ }^{89}$ und gewisse Eingriffsrechte ${ }^{90} .91$

Empfohlen ist schließlich auch, die Frage der Abstraktheit oder Kausalität vom Grundgeschäft ausdrücklich zu klären. Zwar spricht sich der Bundesrat an einer Stelle in der Botschaft dafür aus, die Verfügung über ein Registerwertrecht als „grundsätzlich“ kausal zum Verpflichtungsgeschäft zu halten. ${ }^{92}$ Indes sind solche Gesetzgebungsmaterialien keine verbindlichen Rechtsquellen. Zudem erläutert die Botschaft ihre Position nicht, sondern verweist lediglich auf die überwiegend befürwortenden Stellungnahmen aus der Vernehmlassung sowie einen einzelnen Beitrag aus der Lehre. ${ }^{93}$ Letzterer beruft sich auf die Analogie zum klassischen Wertpapierrecht und fürchtet bei Abstraktheit „erhebliche Einschränkungen“ namentlich im Bereich der Verwahrung von Registerwertrechten und deren Handel, weil Registerwertrechte „oftmals auf die Adresse eines Verwahrerers oder Handelsplatzes übertragen werden dürften, ohne dass damit ein Rechtsübergang beabsichtigt ist“. ${ }^{94}$

\footnotetext{
82 Botschaft (Fn. 3), S. 276.

83 SBF (Fn. 61), Zirkular 2021/01, S. 10.

${ }^{84}$ Botschaft (Fn. 2), S. 276. Letztere Vorgänge erfordern die Zustimmung aller Beteiligten.

85 Vgl. von der Crone/Derungs (Fn. 6) 491.

86 Vgl. von der Crone/Monsch/Meisser (Fn. 17), S. $491 \mathrm{f}$.

87 Dazu Kuhn/Stengel/Meisser/Weber (Fn. 62), Rn. 45.

${ }^{88}$ Vgl. von der Crone/Derungs (Fn. 6), S.491; von der Crone/Monsch/Meisser (Fn. 17),

89 Botschaft (Fn. 2), S. 289.

90 SBF (Fn. 61), Zirkular 2021/01, S. 4: Pausieren des gesamten Registers, White-Listing, Einfrieren einzelner Adressen und Zurückholen verlorener Token.

91 SBF (Fn. 61), Zirkular 2021/01, S. 10.

92 Die Botschaft (Fn. 2), S. 286.

93 Botschaft (Fn. 2), S. 286.

${ }^{94}$ Kramer et al (Fn. 37), Rn. 28.
}

S. 13. 
Unerwähnt bleibt die Tatsache, dass unter dem Bucheffektengesetz seit über 10 Jahren Verfügungen abstrakt erfolgen. Zudem ist in Liechtenstein das Abstraktionsprinzip inzwischen seit gut eineinhalb Jahren in Kraft, ohne dass sich dort die von den Befürwortern des Kausalitätsprinzps ins Feld geführten Befürchtungen manifestiert hätten. Schließlich war sich jedenfalls die Expertenkommission, der auch die Verfasserin angehörte, in der Frage nicht einig und hat daher bewusst von einer entsprechenden Antwort im Gesetzesentwurf abgesehen - wohl zu Recht. Denn der Entscheid über Abstraktion und Kausalität kann nicht isoliert getroffen werden, sondern ist in einen Gesamtkontext mit anderen Wertungsentscheiden zu stellen. Letztlich geht es um die Frage, wie liquide ein Vermögenswert ausgestaltet werden bzw. welche Umlauffähigkeit ihm zugestanden werden soll. Es gilt abzuwägen zwischen den Interessen des Geschäftsverkehrs auf der einen Seite und der Schutzwürdigkeit einzelner Betroffener auf der anderen Seite. Diesen Anforderungen wird jedenfalls der Hinweis in der Botschaft nicht ausreichend gerecht, so dass um der Rechtssicherheit willen die Registrierungsvereinbarung zusätzlich eine eigene Regelung enthalten sollte.

\section{Registrierung}

Die mit der Registrierungsvereinbarung getroffene Verknüpfung muss schließlich noch technisch vollzogen werden, da andernfalls eine Verfügung über das im Wertrechteregister abzubildende Recht verunmöglicht würde. Folglich braucht es also auch einen entsprechenden Eintrag bzw. eine Registrierung (Art. 973d Abs. 1 Ziff. 1 OR). Erst damit gelangt das Registerwertrecht zur Entstehung.

\section{Weitere, nicht konstitutive Anforderungen}

Je länger der Katalog der konstitutiven Voraussetzungen an das Wertrechteregister ausfällt, desto größer ist die Gefahr, dass im konkreten Fall zumindest eine der Voraussetzungen nicht erfüllt wird und dadurch die gültige Entstehung von Registerwertrechten und deren formlose Übertragung nach den Bestimmungen des Gesetzes verhindert. Dem hat der Gesetzgeber mit einer Beschränkung der konstitutiven Elemente Rechnung getragen.

Gleichzeitig sind weitere Anforderungen praktisch ebenso essentiell, so etwa die zweckmäßige Organisation und die jederzeitige Funktionsfähigkeit des Registers. Um deren Umsetzung gesetzlich dennoch zu fördern, statuiert Art. 973d Abs. 3 OR nun entsprechende Pflichten des Schuldners - verstärkt mit der weiteren Pflicht, jedem Erwerber Auskunft zu erteilen über den Inhalt des Registerwertrechts, zur Funktionsweise und Integrität des Wertrechteregisters sowie den diesbezüglichen Schutzmaßnahmen (Art. 973i Abs.1 OR). Eine Verletzung dieser Pflichten macht nicht etwa ein Registerwertrecht oder seine 
Transaktion ungültig, sondern ruft eine - nicht abdingbare - Sorgfaltshaftung hervor (Art. 973 i Abs. 2 und 3 i. V.m. Art. 97 OR). ${ }^{95}$

\section{Wirkungen}

Die privatrechtlichen Wirkungen der Registerwertrechte können nach zwei Richtungen hin unterschieden werden: einerseits, mit Blick auf die Rechte am Registerwertrecht, gegenüber Dritten, und andererseits, mit Bezug auf die Rechte aus dem Registerwertrecht, gegenüber dem Schuldner.

\section{Rechte am Registerwertrecht}

\section{a) Statisch (Abwebr und Auskebr)}

Gegenüber Dritten zeigen sich die Wirkungen der Registerwertrechte in zweierlei Hinsicht. Einerseits statisch, als Abwehr- und Auskehrrecht und andererseits dynamisch, bei der Übertragung oder anderweitigen Verfügung (insb. Belastung mit Sicherungsrechten). Die mit einem Registerwertrecht verbundene statische Rechtsposition entfaltet gewisse Wirkungen erga omnes, die jenen des Eigentums nahekommen. Gerechtfertigt ist dies, weil die den Registerwertrechten unterliegende Technologie ihrem Inhaber eine rivale und exklusive Verfügungsmacht verschafft. ${ }^{96}$ Das nähert sie im Hinblick auf die Beherrschbarkeit den Sachen, an denen eine dingliche, absolute Rechtsposition begründet werden kann, weitgehend an.

Einen eigentlichen Lackmustest für die Tragweite solcher absoluter Rechtspositionen stellt das Insolvenzverfahren dar. Dort regelt seit dem 1. August 2021 der neue Art. 242a SchKG ${ }^{97}$ eine Art Herausgaberecht gegenüber dem konkursiten Verwahrer ${ }^{98}$ von sog. kryptobasierten Vermögenswerten. ${ }^{99}$ Letzterer Begriff erfasst auch, aber nicht nur Registerwertrechte. Ihm unterfallen alle Vermögenswerte, „bei denen die Verfügungsmacht ausschließlich über ein kryptobasiertes Zugangsverfahren vermittelt wird “. ${ }^{100}$ Hier nun sind auch kryptobasierte Zahlungsmittel (d.h. Vermögenswerte, die „tatsächlich oder nach der Absicht des Organisators oder Herausgebers in einem erheblichen Umfang als Zahlungs-

95 Botschaft (Fn. 2), S. 278; Weber(Fn. 56), Rn. 18.

96 Vgl. Fn. 64 und 65.

97 Bundesgesetz über Schuldbetreibung und Konkurs (11. April 1889), SR 281.1.

$98 \mathrm{Zu}$ möglichen Ausgestaltungen der Verwahrungsverhältnisse Kramer/Wyss, Verwahrung von digitalen Aktiven, in: Weber/Kuhn (Hrsg.) Entwicklungen im Schweizer Blockchain-Recht (2021), S. 145, Rn. 8 ff. Zum wirtschaftlichen Hintergrund der Verwahrungsverhältnisse Botschaft (Fn.2), S. 233 ff.; Kramer/Meier, Tokenisierung von Finanzinstrumenten, GesKR 2020, 60, 72.

99 Vgl. Weber (Fn. 56), Rn. $32 \mathrm{ff}$.

100 Botschaft (Fn. 2), S. 292. 
mittel für den Erwerb von Waren oder Dienstleistungen oder der Geld- oder Wertübertragung dienen“) ${ }^{101}$ in jedem Fall und unabhängig von der Existenz eines individualisierbaren Emittenten miterfasst.

Als Verwahrer (z. B. Wallet Provider) bzw. sog. crypto custodians gilt nach Art. 242a Abs. 2 pr SchKG und in Anlehung zur Hinterlegung nur, wer sich verpflichtet, die kryptobasierten Vermögenswerte nach Zahl und Gattung ununterbrochen und jederzeit in seinem selbstständigen Gewahrsam (d.h. als alleiniger Inhaber) für den Berechtigten bereitzuhalten. ${ }^{102}$ Die Verwendung der kryptobasierten Vermögenswerte im Rahmen des Aktivgeschäfts (wie bei Bankeinlagen) ist ihnen damit von vornherein verwehrt. ${ }^{103}$

Der Konkursit ist hier kraft seiner Rolle zwar Inhaber der kryptobasierten Vermögenswerte, nicht aber der an ihnen Berechtigte. Daher erlaubt Art.242a Abs. 2 SchKG dem Berechtigten, die Aussonderung, ${ }^{104}$ soweit kryptobasierte Vermögenswerte inner- oder außerhalb des Registers dem Berechtigten entweder individuell zugeordnet sind (Bst. a) oder zwar nur einer Gemeinschaft zugeordnet sind, aber der Anteil des Berechtigten am Gemeinschaftsvermögen ermittelt werden kann (Bst. b). Will die Konkursverwaltung die Aussonderung als unbegründet verweigern, setzt sie dem Berechtigten Frist von 20 Tagen, innert deren er auf Aussonderung klagen kann, andernfalls seine behauptete Berechtigung verwirkt (Abs. 3). ${ }^{105}$ Für die Absonderung kryptobasierter Vermögenswerte aus der Insolvenzmasse prudenziell regulierter und beaufsichtigter Verwahrer (Bank, Wertpapierhaus, Fondsleitung und Finanzmarktinfrastruktur) greifen (inhaltlich übereinstimmende) Sonderregeln. ${ }^{106}$

Außerbalb der Insolvenz hat der Gesetzgeber bemerkenswerterweise darauf verzichtet, dem Berechtigten Rechtsbehelfe zur Abwehr und Auskehr einzuräumen, welche der Negatorienklage und der Vindikation nach Art. 641 ZGB entsprechen würden. Geregelt ist nur gerade das gerichtliche Kraftloserklärungsverfahren (Art. $973 \mathrm{~h} \mathrm{OR).}{ }^{107}$ Es ist aber kosten- und zeitintensiv

101 So die vorläufige Formulierung im Vernehmlassungsentwurf zu einem neuen Art. 5a Abs. 1 BankV (Verordnung über die Banken und Sparkassen, Bankenverordnung, 30. April 2014, SR 952.02).

102 Botschaft (Fn. 2), S. 263 und 292.

103 Vgl. Zellweger-Gutknecht/Weber (Fn. 55), Rn. $17 \mathrm{ff}$.

104 Während nach schweizerischem Recht die Aussonderung den Regelfall darstellt und erst auf Initiative der Berechtigten erfolgt, sehen diverse Finanzmarktrechte (darunter Art. $17 \mathrm{ff}$. BEG) eine sog. Absonderung vor, bei welcher die Konkursbehörde von Amtes wegen tätig wird.

105 Vgl. im Einzelnen Kramer/Wyss (Fn. 99), Rn. $39 \mathrm{ff}$.

106 Vgl. insb. Art. 16 Ziff. 1 i. V. m. Art.37d BankG (Bundesgesetz über die Banken und Sparkassen, Bankengesetz, 8. November 1934, SR 952.0) und Art. 88 Abs. 1 FinfraG (Bundesgesetz über die Finanzmarktinfrastrukturen und das Marktverhalten im Effekten- und Derivatehandel, Finanzmarktinfrastrukturgesetz, 19. Juni 2015, SR 958.1); Zellweger-Gutknecht/ Weber (Fn. 55), Rn. 19ff.; Kramer/Wyss (Fn. 99), Rn. 43 ff.

107 Dazu Weber (Fn. 56), Rn. 27. 
und grundsätzlich für unbekannte Inhaber ausgelegt (vgl. nur Art. 983 OR). Es verursacht dem Berechtigten, der nach Abschluss des Verfahrens die Ausfertigung neuer Registerwertrechte verlangt, die damit verbundenen Kosten. Diese lassen sich nur abwälzen, soweit sich der unberechtigte Inhaber einer Herausgabe ungerechtfertigt und schuldhaft widersetzt. Vorausgesetzt ist zunächst, dass die ursprüngliche Inhaberschaft (im Gesetz als „Verfügungsmacht“ und in der Botschaft als Entsprechung zum Besitz bezeichnet) sowie deren Verlust (etwa Abhandenkommen des Private Key im Zuge eines Erbgangs) glaubhaft gemacht werden (Art. 973h Abs. 1 Satz 1 OR). ${ }^{108}$ Nach der Kraftloserklärung kann gegenüber dem Schuldner entweder das unterliegende Recht außerhalb des Registers geltend gemacht oder die Ausgabe eines neuen Registerwertrechts auf Kosten des Berechtigten verlangt werden (Satz 2).

Wird im Falle von Wertpapieren die Urkunde vorgelegt, muss der Berechtigte zudem auf deren Herausgabe klagen (Art. 985 OR). Da Art.973h OR die Art. 981-986 OR für sinngemäß anwendbar erklärt, wäre bei einem bekannten, unberechtigten Inhaber folgendes Vorgehen denkbar: Das Gericht fordert ihn statt durch öffentliche Bekanntmachung direkt auf, das Registerwertrecht innerhalb bestimmter Frist „vorzulegen“. Bei Registerwertrechten läuft das sinngemäß auf die einstweilige Sistierung der Inhaberschaft („Einfrieren“) hinaus; etwa durch entsprechende Anordnung des Gerichts an den Schuldner. Alsdann setzt das Gericht dem Berechtigten Frist, um gegen den Inhaber Klage auf Übertragung des Registerwertrechts zu erheben und auferlegt nach dem Vorbild von Art. 982 OR dem Schuldner gegebenenfalls ein einstweiliges Leistungsverbot.

Alternativ dazu wird in der Literatur ein Vorgehen des Schuldners nach den Regeln des Bereicherungsrechts (Art. 62 ff. OR) vorgeschlagen. ${ }^{109}$ Die Kondiktion als subsidiärer Rechtsbehelf wird indes nur offenstehen, wenn das soeben skizzierte Vorgehen sich als nicht gangbar erweisen sollte. Zudem hat es im Gegensatz zum Kraftloserklärungsverfahren den Nachteil der relativen und absoluten Befristung (Art.67 Abs. 1 OR) und ist insofern von den unbefristeten Eigentümerabwehrrechten weiter entfernt.

Sollte der unterlegene Inhaber in der Vollstreckung (Art.343 ZPO) die Übertragung weiterhin verweigern, wäre dem Kläger mit den Rechtsfolgen der Kraftloserklärung (unter Kostenüberwälzung auf den Inhaber) besser gedient. Da der Katalog von Art. 343 ZPO nicht abschließend ist, ${ }^{110}$ sollte das Gericht sinngemäß nach Art. 986 OR vorgehen, d.h. das Registerwertrecht obne Veröffentlichung für kraftlos erklären und die Kosten der allfälligen Neuausfertigung dem bisherigen Inhaber überbinden dürfen.

108 Botschaft (Fn. 2), S. 288.

109 Kubn (Fn. 12), Rn. 46 m. w. H.

110 Bundesrat, Botschaft zur Schweizerischen Zivilprozessordnung (ZPO), BBl. 2006 7221, S. 7385. 


\section{b) Dynamisch (Verfügung)}

Wie eingangs bereits dargelegt, wird das im Informationsmedium „Registerwertrecht" abgebildete Recht umlauffähiger, weil es, bedingt durch die in der Registrierungsvereinbarung begründete Verknüpfung, dem Recht am Medium folgt und insofern von dessen Privilegien punkto Transport, Legitimation und Verkehrsschutz profitiert. Einige dieser privilegierenden Wirkungen sind nachfolgend dargestellt.

Ist ein Registerwertrecht begründet, kann es prinzipiell nur noch auf dem Register und nach den Regeln der Registrierungsvereinbarung übertragen werden (Art. 973d Abs. 1 Ziff. 2, Abs. 2 Ziff. 3 sowie 973f Abs. 1 OR). Eine Ausnahme greift nur, wenn - abredewidrig - neben der Registrierung auch ein Wertpapier ausgestellt wurde: Hier geht der gutgläubige Erwerb des Wertpapiers dem gutgläubigen Erwerb des Registerwertrechts vor (Art. 973f Abs. 3 OR). ${ }^{111}$ Entsprechend obliegt es dem Schuldner, in der Registrierungsvereinbarung den Verfügungsmechanismus und dessen Wirkungen möglichst genau zu definieren. ${ }^{112}$

Das Gesetzt regelt in dieser Hinsicht nur gerade den Zeitpunkt der Finalität für den Fall, dass über einen Gläubiger der Konkurs eröffnet, die Pfändung vollzogen oder die Nachlassstundung bewilligt wird: Nach Art.973f Abs. 2 OR belasten Verfügungen, die im Zeitpunkt der Eröffung des Zwangsvollstreckungsverfahrens noch nicht vollständig abgeschlossen waren, das Vermögen des Gläubigers nur dann, wenn sie vor Verfahrenseröffnung eingebracht (Ziff. 1), innert 24 Stunden im Register tatsächlich eingetragen (Ziff. 3) und bis zu letzterem Zeitpunkt nach den Regeln des Registers oder eines übergeordneten Handelssystems unwiderruflich wurden (Ziff. 2). ${ }^{113}$ Soweit die rechtsgeschäftliche Übertragung kausal ausgestaltet wird, ${ }^{114}$ braucht es zusätzlich ein gültiges Grundgeschäft. ${ }^{115}$

Unanwendbar bleiben müssen hingegen die Normen betreffend die Verfügung über andere qualifizierte Informationsmedien, so namentlich die auf Inhaber oder an Order ausgestellte Wertpapiere. Entsprechend ist es verfehlt, für die auf Order gestellten Registerwertrechte eine lückenlose Indossamentenkette vorauszusetzen. Richtig ist hingegen, dass an deren Stelle die Registrierung in einem Wertrechteregister getreten ist, ${ }^{116}$ das dank seiner konstitu-

111 Der Inhaber des Registerwertrechts wird sich dann gestützt auf Art. 973i OR an den Schuldner wenden.

112 Vgl. dazu III.3. Registrierungsvereinbarung.

113 Vgl. (inkl. Finalität nach finanzmarktrechtlichen Bestimmungen) Weber, Handel mit digitalen Aktiven, in: ders./Kuhn (Hrsg.), Entwicklungen im Schweizer Blockchain-Recht (2021), S. 165, Rn. $95 \mathrm{ff}$.

${ }^{114}$ Vgl. dazu III.3. Registrierungsvereinbarung i.f.

115 Botschaft (Fn. 2), S.286.

116 So zutreffend Botschaft (Fn. 2), S. 285. 
tiven Eigenschaften für den Legitimationsnachweis vergleichbare Gewähr zu leisten vermag wie die Indossamentenkette. Anders als beim Indossament hat hier indes der Inhaber die Lückenlosigkeit nicht zu dokumentieren, sondern wird diese kraft entsprechender Rechtsvermutung in Art. 973e Abs. 2 OR unterstellt und dem Schuldner der Gegenbeweis anheimgestellt. Daran ändert auch Art. 684 Abs. 2 OR nichts, der für die rechtsgeschäftliche Übertragung von Namenaktien die „Übergabe des indossierten Aktientitels an den Erwerber“ verlangt. Denn während etwa Art. 683 Abs. 1 OR, der die Ausgabe von Inhaberaktien vor deren vollständigen Liberierung untersagt, gesellschaftsrechtlich motiviert ist und damit den wertpapierrechtlichen Normen der Art. $965 \mathrm{ff}$. OR zwingend vorgeht, fehlt Art. 684 Abs. 2 OR eine vergleichbare, im Aktienrecht selbst gründende Ratio. Die fragliche Norm ist vielmehr reine Wiederholung der wertpapierrechtlichen Bestimmungen zum Transfer von Orderpapieren und wird daher, falls eine Namenaktie statt in einem Wertpapier in einem Registerwertrecht abgebildet wird, durch Art. 973f OR vollständig derogiert. Entsprechend ist eine Bestimmung in der Registrierungsvereinbarung, wonach die Übertragung nach den Regeln des Systems „als Indossament qualifiziert“117 nicht nur unnötig, sondern vielmehr widersprüchlich, wenn nicht gar gefährlich, da sie die Beweislast zulasten der Inhaber neu verteilen könnte.

Weiter kann ein Inhaber über ein Registerwertrecht auch verfügen, indem er es belastet bzw. etwa als Sicherheit verwendet oder eine Nutznießung daran einräumt (Art.973g OR). Das Gesetz wählt hier einen zweigleisigen Ansatz. Es definiert primär funktional für das Pfandrecht, dass das Wertrechteregister zum einen die Belastung sichtbar machen muss (Abs. 1 Ziff. 1) und zum andern ausschließlich dem aus der Belastung Berechtigten Verfügungsmacht über das Registerwertrecht einräumt (Ziff. 2). Subsidiär bzw. „im Übrigen“ erklärt es sodann bestehende Normen für anwendbar: für die Retention die Art. 895-898 ZGB und für das Pfandrecht die Art. 899-906 ZGB ${ }^{118}$ (ohne Art. 900 Abs. 1 ZGB). Die Nutznießung bestimmt sich (auch ohne entsprechenden Verweis) aus Art. 745 ff. ZGB. ${ }^{119}$

Sodann erleichtern gleich mehrere Regeln zum Verkebrsschutz den rechtsgeschäftlichen Erwerb: Nach dem Vorbild des Gutglaubensschutzes bei Ordrepapieren (Art. 1006 Abs. 2 OR) erwirbt der Gutgläubige oder nur leicht Fahrlässige das Registerwertrecht originär auch vom nichtberechtigten Inhaber, weil er im Wertrechteregister als Gläubiger ausgewiesen ist (Art.973e Abs. $3 \mathrm{OR}$ ). Um diese Folge zu verhindern, obliegt es dem Berechtigten, bereits vorgängig das Registerwertrecht vom Gericht kraftlos erklären zu lassen oder die unge-

117 So Kubn (Fn. 12), Rn. 103 und SBF (Fn. 61), Zirkular 2021/01, S. 10.

118 Botschaft (Fn. 2), S. 287.

119 Vgl. zu den Belastungen auch Kubn (Fn. 12), Rn. 47f. 
rechtfertigte Bereicherung beim Nichtberechtigten zu kondizieren (Art. $62 \mathrm{ff}$. OR). ${ }^{120}$

Auch bei den Einreden bestehen verkehrsschützende Einschränkungen, die in Anlehnung an Art. 979 OR (Inhaberpapier) bzw. 1007 OR (Orderpapier) ausgestaltet wurden. So kann der Schuldner Einreden gegen frühere Gläubiger nur vorbringen, wenn der aktuelle Gläubiger das Registerwertrecht bewusst zum Nachteil des Schuldners erworben hat (Art. 973 Abs. 4 Ziff. 3 OR). Im Übrigen sind Einreden einzig zulässig wegen Ungültigkeit der Registrierung oder weil sie aus dem Register oder mit ihm verknüpften Begleitdaten hervorgehen (Ziff. 1) oder persönlich gegen den aktuellen Gläubiger gerichtet sind (Ziff. 2). ${ }^{121}$

\section{Rechte aus dem Registerwertrecht}

Zum Schluss bleibt ein Blick auf die Rechte „aus dem Registerwertrecht“ - in der an das Wertpapierrecht angelehnten Terminologie. Zunächst versteht sich von selbst, dass sie sich nach dem Inhalt des Rechts des Gläubigers gegenüber dem Emittenten richten. Sie können entsprechend vielfältig sein und folglich nicht pauschal behandelt werden. Die hier gestellte Frage nach den Rechten aus dem Registerwertrecht zielt indes auf etwas Anderes. Es geht nicht darum, was gestützt auf ein Registerwertrecht zu leisten ist, sondern wann bzw. unter welchen Bedingungen. Gefragt ist mithin nach den mit diesem Informationsmedium verbundenen Wirkungen zwischen Schuldner und Gläubiger.

Dazu zählt zum einen die Präsentationswirkung gemäß Art. 973e Abs. 1 OR, nach welcher die Leistung in Anlehnung an Art. 965 OR nur an den vom Register ausgewiesenen Gläubiger erfolgen darf und muss. Zudem muss auch die Erfüllung im Register reflektiert werden - etwa indem das Registerwertrecht an den Schuldner übertragen oder als erfüllt geflagt wird (was wiederum vergleichbar ist mit der Pflicht zur Herausgabe der Urkunde nach Tilgung gemäß Art. 966 Abs. 1 OR).

Zum andern greift die Legitimationswirkung nach dem Vorbild von Art. 966 Abs. 2 OR: Der Inhaber des Registerwertrechts gilt als rechtszuständig. Folglich befreit die Leistung an den bei Verfall durch das Register bezeichneten Gläubiger, sofern der Schuldner nicht mindestens grobfahrlässig über dessen Berechtigung irrte (Art. 973e Abs. 2 OR). Ist in Wahrheit ein anderer Gläubiger der Berechtigte, kann er die Leistung nicht ein zweites Mal vom Schuldner verlangen, sondern muss sich an den Inhaber, d. h. den vom Wertrechteregister bezeichneten Gläubiger halten, der die Leistung zu Unrecht erhalten hat (Art.62ff. OR).

Erfüllt ein Wertrechteregister somit die konstitutiven Anforderungen des Art. 973d Abs. 2 OR, so genießen die auf ihm getätigten Einträge, d. h. die Re-

120 Vgl. dazu IV.1.a.

121 Zur Einredenordnung der Registerwertrechte einlässlich Kubn (Fn. 12), Rn. 32. 
gisterwertrechte und darauf bezogene Verfügungen, öffentlichen Glauben genauso wie die ebenfalls privat erstellten Wertpapiere öffentlichen Glaubens.

\section{Ausblick}

Das schweizerische DLT-Mantelgesetz und die damit verbundene Schaffung eines Registerwertrechts darf mit Fug als Meilenstein bezeichnet werden. Doch so pragmatisch der Ansatz und so vergleichsweise schlank die neuen Bestimmungen auf den ersten Blick auch scheinen mögen, sollte deren Tragweite und Wirkung doch nicht unterschätzt werden. Sie reichen bis tief in die Fundamente des Privatrechts und des (vorliegend nicht thematisierten) öffentlichen Finanzmarktrechts hinein und werden in ihrer ganzen Dimension erst nach und nach, durch die Anwendung in der Praxis und entsprechende Rechtsprechung, schärfere Kontur erhalten.

Die Schweiz dürfte zwar zu den ersten Rechtsordnungen zählen, welche nicht nur über punktuelles Enforcement, sondern mittels Gesetzgebung Raum geschaffen haben für eine rechtssichere Anwendung und inovative Entwicklung der noch jungen Technologie der verteilten und ähnlich manipulationsresistenten Register und darauf gebuchter Vermögenswerte. Dennoch sollte sie sich auf dem Erreichten nicht ausruhen.

Gerade der grenzüberschreitende Geschäftsverkehr verlangt nach Lösungen, welche die Interoperabilität über die Landesgrenzen hinweg gewährleisten. So wie bereits die FINMA mit ihrem Versuch einer Kategorisierung von kryptobasierten Vermögenswerten prägenden Einfluss auf andere Behörden genommen hat, ${ }^{122}$ ist zu hoffen, dass überzeugende Ansätze der Schweizer DLT-Gesetzgebung ausländische Legislativen und Regulatoren inspirieren und damit zu einer graduellen Rechtsangleichung beitragen mögen. Gleichzeitig muss aber auch die Schweiz die internationale Entwicklung aufmerksam verfolgen und darf nicht zögern, dort nachzubessern, wo entsprechender Bedarf sichtbar wird und überzeugendere Lösungen im Ausland vielleicht gar schon in Kraft stehen.

Größte Herausforderungen bilden hier zum einen die (primär aufsichts-) rechtliche Einbettung der vollkommen dezentralisierten Finanztechnologien (DeFi). Zum andern sollte alles daran gesetzt werden, ein Auseinanderdriften der nationalen dogmatischen Konzepte zur Erfassung kryptobasierter Vermögenswerte und sie betreffende Verfügungen nach dem „Vorbild“ der interme-

122 Vgl. nur American Bar Association, Derivatives and Futures Law Committee, Innovative Digital Products and Processes Subcommittee, Jurisdiction Working Group, Digital and Digitized Assets: Federal and State Jurisdictional Issues, 2019, 26, welche auf die FINMA-Kategorisierung nicht nur explizit referenziert, sondern sie auch übernimmt sowie die bei $K u b n$ (Fn. 12), Fn. 9 genannten weiteren ausländischen Materialien. 
diatisierten Wertpapiere zu vermeiden. ${ }^{123}$ Kollaborative Projekte wie jenes von UNIDROIT zu „Digital Assets and Private Law“ leisten hier wertvolle Arbeit und sollten durch Politik und Wissenschaft nach Kräften unterstützt und gefördert werden.

123 Anschaulich Paech, The Impact of the Geneva Securities Convention and the Future European Legislation, in: Conac/Segna/Thévenoz (Hrsg.), Intermediated Securities (2013), S.22, https://doi.org/10.1017/CBO9781139151238.004. 
Teil 2

Regelungsvorschläge 



\title{
Die allgemeinen privatrechtlichen Regelungen für elektronische Wertpapiere
}

\author{
National und international
}

Dorothee Einsele

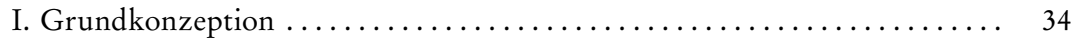

II. Entstehung der Verpflichtung des Emittenten aus dem

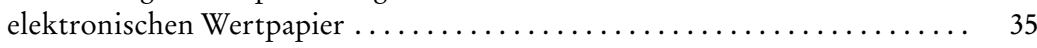

III. Legitimationsfunktion der Registereintragung zugunsten des Inhabers . . . . 36

1. Formelle Legitimation des Inhabers $\ldots \ldots \ldots \ldots \ldots \ldots \ldots \ldots \ldots \ldots \ldots \ldots$

2. Vermutung für materielle Berechtigung des Inhabers? .......... 38

a) Elektronische Wertpapiere in Einzeleintragung ............ 38

b) Elektronische Wertpapiere in Sammeleintragung............ 38

3. Ausnahme: Fehlende (Verfügungs-)Berechtigung des Inhabers . . . . . . 39

4. Legitimation des Hinterlegers durch Depotbescheinigung .......... 40

IV. Liberationsfunktion der Registereintragung zugunsten des Emittenten ... . 42

V. Einwendungen des Emittenten und Einwendungsausschluss ......... 43

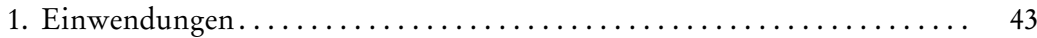

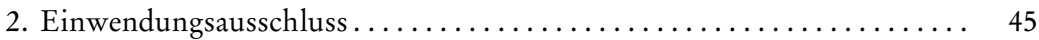

VI. Verkehrsschutzfunktion durch gutgläubigen Erwerb ............ 46

1. Elektronische Wertpapiere in Sammeleintragung ............. 46

2. Elektronische Wertpapiere in Einzeleintragung ............. 48

3. Problem: Verfügungen über Mischbestand aus elektronischen
Wertpapieren in Sammeleintragung und Einzeleintragung ........ 50

VII. Fiktion des elektronischen Wertpapiers als Sache .............. 51

1. Elektronische Wertpapiere in Sammeleintragung .............. 51

2. Elektronische Wertpapiere in Einzeleintragung $\ldots \ldots \ldots \ldots \ldots \ldots \ldots . \ldots 3$

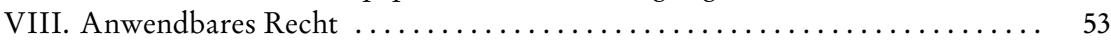

1. Elektronische Wertpapiere in Einzeleintragung .............. 53

2. Elektronische Wertpapiere in Sammeleintragung ............. 56

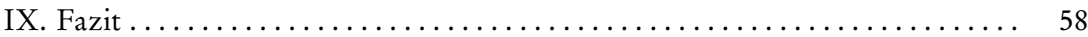




\section{Grundkonzeption}

Nach dem Gesetz zur Einführung von elektronischen Wertpapieren ${ }^{1}$ sollen Inhaberschuldverschreibungen auch als elektronische Wertpapiere begeben werden können (Art. 1 Gesetz über elektronische Wertpapiere, im Folgenden eWpG). Damit beschränkt das Gesetz seinen Anwendungsbereich im Grundsatz $^{2}$ auf Inhaberschuldverschreibungen, die - soweit verbrieft - in den $\$ \$ 793 \mathrm{ff}$. BGB geregelt sind. An Stelle der Kreation, also der Ausstellung einer Wertpapierurkunde, tritt bei elektronischen Wertpapieren die Eintragung in das Wertpapierregister ( $\$ 2$ Abs. $1 \mathrm{eWpG}$ ). Dabei soll sich das elektronische Wertpapier nur durch die Begebungsform von einem mittels Papierurkunde begebenen Wertpapier unterscheiden. ${ }^{3}$ Insbesondere soll ein elektronisches Wertpapier gem. $\$ 2$ Abs. 2 eWpG im Grundsatz dieselbe Rechtswirkung wie ein Wertpapier entfalten, das mittels Urkunde begeben worden ist. Im Rahmen der Regelungen zu elektronischen Wertpapieren nimmt das eWpG indes zwei wesentliche Unterscheidungen vor:

Zum einen differenziert das Gesetz zwischen den in einem zentralen Register eingetragenen Zentralregisterwertpapieren ( $\mathbb{S} 4$ Abs.1 Nr.1, Abs.2, 12 eWpG) und den in das dezentrale Kryptowertpapierregister eingetragenen Kryptowertpapieren ( $\mathbb{S} 4$ Abs. 1 Nr. 2, Abs. 3, 16 eWpG). In beiden Fällen handelt es sich zwar um elektronische Wertpapiere, für die aber teils unterschiedliche Regelungen zur Anwendung gelangen. Dies betrifft zunächst das jeweilige Register, in dem die elektronischen Wertpapiere geführt werden. So hat das Kryptowertpapierregister in den $\$ \$ 16$ bis $23 \mathrm{eWpG}$ eine Regelung erfahren, die von den Bestimmungen der $\$ \$ 12$ bis $15 \mathrm{eWp}$ für zentrale Wertpapierregister abweicht. Insbesondere trifft den Emittenten bei Kryptowertpapierregistern eine Garantenpflicht hinsichtlich des ordnungsgemäßen Funktionierens des Kryptowertpapierregisters sowie der Integrität und Authentizität der Kryptowertpapiere $(\$ 21 \mathrm{eWpG})$. Diese Pflicht des Emittenten ist ebenso zwingender Inhalt der elektronischen Schuldverschreibung wie auch das Recht des Inhabers, die Schuldverschreibung außerordentlich zu kündigen, ${ }^{4}$ falls der Emittent die Funktionstüchtigkeit des Kryptowertpapierregisters nach Setzung einer angemessenen Frist nicht wiederherstellt $(\$ 30 \mathrm{eWpG})$.

${ }^{1}$ Gesetz vom 3.6.2021, BGBl. 2021 I, S. 1423.

2 Vgl. zur Möglichkeit, auch Investmentanteilscheine in elektronischer Form zu begeben, Art. 10 des Gesetzes zur Einführung von elektronischen Wertpapieren, wonach auch $\mathbb{9 5}$ KAGB geändert wird; vgl. auch dazu, dass Investmentanteilscheine nach h. M. als Wertpapiere eigener Art und nicht (nur) als Inhaberschuldverschreibungen eingeordnet werden, Einsele, Bank- und Kapitalmarktrecht - Nationale und Internationale Bankgeschäfte (4. Aufl. 2018), \$10 Rn. 28.

3 Vgl. Begründung RegE zu $\$ 2$ Abs. 2, BT-Drs. 19/26925, S. 40.

4 Vgl. zur dogmatischen Einordnung der Kündigungsrechte der Inhaber von Schuldverschreibungen als forderungsbezogene Gestaltungsrechte, Bialluch, Das sogenannte Anleiheschuldverhältnis (2018), S. 60 m. w. N. 
Zum anderen differenziert das eWpG zwischen elektronischen Wertpapieren in Sammeleintragung und solchen in Einzeleintragung. Die Möglichkeit der Sammeleintragung auf den Namen einer Wertpapiersammelbank bzw. eines sonstigen Verwahrers besteht für alle elektronischen Wertpapiere $(\mathbb{S} 8$ Abs. 1 Nr. 1 eWpG). Auch können nach dem eWpG - anders als noch im RefE vorgesehen ${ }^{5}$ - sowohl Zentralregisterwertpapiere als auch Kryptowertpapiere für den Berechtigten in Einzeleintragung geführt werden ( $\$ 8$ Abs. 1 Nr.2 eWpG).

Dabei unterscheidet das eWpG zwischen dem im elektronischen Wertpapierregister eingetragenen Inhaber des elektronischen Wertpapiers ( $\$ 3$ Abs. 1 $\mathrm{eWp}$ ) und dem Berechtigten, der das Recht aus einem Wertpapier innehat ( $\$ 3$ Abs. 2 eWpG). Im Fall der Sammeleintragung wird zwar die Wertpapiersammelbank oder ein sonstiger Verwahrer als Inhaber in das Wertpapierregister eingetragen ( $\$ 8$ Abs. 1 Nr. 1 eWpG). Bei der Wertpapiersammelbank bzw. dem Verwahrer handelt es sich aber gerade nicht um die Berechtigten, also die Inhaber des Rechts aus dem Wertpapier. ${ }^{6}$ Anders ist dies bei der Einzeleintragung, bei der der materiell Berechtigte in das Wertpapierregister als Inhaber eingetragen ist.

Wie noch näher zu zeigen sein wird, gelten für elektronische Wertpapiere in Einzeleintragung und solche in Sammeleintragung recht unterschiedliche rechtliche Regelungen: So sollen Verfügungen über elektronische Wertpapiere in Sammeleintragung den bisherigen Vorschriften sammelverwahrter Urkunden folgen ( $\$ 9$ Abs. $1 \mathrm{eWpG}$ ). Hingegen finden sich für Verfügungen über elektronische Wertpapiere in Einzeleintragung, und zwar nur für diese, in den $\$ \$ 24$ bis $27 \mathrm{eWpG}$ etliche Sonderregelungen.

\section{Entstehung der Verpflichtung des Emittenten aus dem elektronischen Wertpapier}

Zur Einordnung des Gesetzes zur Einführung elektronischer Wertpapiere in das allgemeine Privatrecht ist zunächst die Frage relevant, welcher Rechtsakt die Verpflichtung des Emittenten aus dem elektronischen Wertpapier begründet. Hinsichtlich der damit angesprochenen Wertpapierrechtstheorien ${ }^{7}$ bezieht das eWpG indes keine Stellung. Insoweit regelt $\$ 2$ Abs. 1 S. 2 eWpG zunächst, dass ein elektronisches Wertpapier dadurch begeben wird, dass der Emittent

5 Vgl. noch $₫ 8$ Abs. 1 RefE.

6 Vgl. insbes. $\$ 9$ Abs. 1 und Abs. 2 eWpG.

7 Vgl. hierzu, insbes. zu der durch die Rechtsscheintheorie modifizierten Vertragstheorie, die Kreationstheorie sowie die Theorie vom mehrgliedrigen Rechtsgeschäft MüKo/Habersack, BGB, Bd.7 (8. Aufl. 2020), Vor $\$ 793$ Rn.26ff.; Baumbach/Hefermehl/Casper, Wechselgesetz, Scheckgesetz, Recht des Zahlungsverkehrs (24. Aufl. 2020), WertpapierR Rn. $29 \mathrm{ff}$. 
an Stelle der Ausstellung einer Wertpapierurkunde die Eintragung in ein elektronisches Wertpapierregister bewirkt. Damit ist aber noch nicht gesagt, dass das eWpG der sog. Kreationstheorie folgen will. Zum einen ist in $\$ 2$ Abs. 1 und Abs. 2 eWpG immerhin auch von der Notwendigkeit der Begebung des elektronischen Wertpapiers die Rede. Zum anderen wird in der Begründung des RegE hervorgehoben, dass dadurch keine normativen Festlegungen im Bereich der Theorien über die Begebung von Wertpapieren getroffen werden sollen. ${ }^{8}$ Demgemäß verzichtet das eWpG denn auch auf eine nähere rechtliche Einordnung dieser Begebung entweder als lediglich faktisches Element oder als sachenrechtlicher Vertrag oder aber als schuld- und sachenrechtlicher Vertrag. Allerdings ist auf eines hinzuweisen: Auch wenn bei elektronischen Wertpapieren die Ausstellung der Wertpapierurkunde durch die Eintragung im elektronischen Wertpapierregister ersetzt wird, ist für die voll wirksame Entstehung der Verpflichtung des Emittenten aus dem elektronischen Wertpapier zusätzlich erforderlich, dass der Berechtigte aus dem Wertpapier feststeht. Denn ohne einen Berechtigten, also denjenigen, der Inhaber des Rechts aus dem Wertpapier ist (vgl. \$3 Abs. 2 eWpG), würde es sich - unabhängig von der favorisierten Wertpapierrechtstheorie - um eine Forderung ohne Gläubiger handeln. ${ }^{9}$

Inhaber des Rechts aus dem Wertpapier ist bei der Sammeleintragung aber nicht die als Inhaber im Wertpapierregister eingetragene Wertpapiersammelbank oder ein sonstiger Verwahrer, sondern der (Erst-)Erwerber des elektronischen Wertpapiers. Daher schließt der Aussteller den Begebungsvertrag nicht mit der Wertpapiersammelbank als im Wertpapierregister eingetragene Inhaberin, sondern mit dem Ersterwerber des Wertpapiers ab. ${ }^{10}$

\section{Legitimationsfunktion der Registereintragung zugunsten des Inhabers}

\section{Formelle Legitimation des Inhabers}

Im Rahmen der Legitimationsfunktion von Inhaberwertpapieren wird der Besitz der Urkunde durch die Eintragung in das elektronische Wertpapierregister ersetzt. Dabei ist im elektronischen Wertpapierregister nur der Inhaber $(\mathbb{8} 8$ Abs. 1 Nr. 1 eWpG), nicht aber der materiell Berechtigte eingetragen. Allerdings ist im Fall der Einzeleintragung der Inhaber zugleich diejenige natürliche oder

\footnotetext{
8 Begründung RegE zu $\$ 2$ Abs. 1, BT-Drs. 19/26925, S. 39.

9 Vgl. auch Hueck/Canaris, Recht der Wertpapiere (12. Aufl. 1986), $\$ 24$ III 3; Zöllner, Wertpapierrecht (14. Aufl. 1987), \$3 I 1a.

10 Auch die Stellungnahme der Deutschen Bundesbank v. 10.9.2020 zum RefE, 3 geht davon aus, dass der Begebungsvertrag jedenfalls bei Sammeleintragung nicht mit dem formell legitimierten Inhaber, sondern dem Ersterwerber der elektronischen Wertpapiere zustande kommt.
} 
juristische Person oder rechtsfähige Personengesellschaft, die das Wertpapier als Berechtigte hält ( $\$ 8$ Abs. 1 Nr. $2 \mathrm{eWpG})$. Gegenüber dem Emittenten formell legitimiert ist indes gem. $\$ 28$ Abs. $1 \mathrm{~S} .1 \mathrm{eWpG}$ nur der eingetragene Inhaber. ${ }^{11}$ So kann der als Inhaber eines elektronischen Wertpapiers oder eines bestimmten Anteils an einer Gesamtemission im elektronischen Wertpapierregister Eingetragene die in der Schuldverschreibung versprochene Leistung verlangen. Die Regelung zur formellen Legitimation entspricht also zunächst $\$ 793$ Abs. 1 S. 1 BGB für verbriefte Inhaberschuldverschreibungen.

Entsprechend $\$ 797$ S. 1 BGB bei verbrieften Inhaberschuldverschreibungen ist der Aussteller zur Leistung aus der elektronischen Schuldverschreibung nur verpflichtet, wenn der Inhaber gegenüber der registerführenden Stelle eine Weisung zur Umtragung ${ }^{12}$ auf den Emittenten bei Zahlungsnachweis erteilt $(\mathbb{\$} 29$ Abs. 1 eWpG). Da der Aussteller mit Umtragung neuer Inhaber der elektronischen Schuldverschreibung wird ( $\ 4$ Abs. 8 eWpG), ist damit der Aussteller elektronischer Wertpapiere - ebenso wie der Aussteller verbriefter Schuldverschreibungen - gegen das Risiko einer mehrfachen Inanspruchnahme geschützt. ${ }^{13}$

Weniger verständlich ist allerdings, dass für die Frage des Erlöschens des Anspruchs aus der Schuldverschreibung nach Ablauf der Vorlegungsfrist sowie für die Verjährung des Leistungsanspruchs nach Vorlegung - mithin im Rahmen des $\$ 801$ BGB - bei elektronischen Wertpapieren nicht nur ein ausdrückliches Leistungsverlangen gefordert wird, sondern derjenige, der dies verlangt, seine Berechtigung auch glaubhaft machen muss (\$29 Abs. 2 eWpG). Die Notwendigkeit, seine Berechtigung glaubhaft zu machen, erscheint allerdings nicht unbedingt kompatibel mit der Regelung, wonach gegenüber dem Emittenten nur der Inhaber formell legitimiert ist. ${ }^{14}$ Daher können andere Personen als die formell legitimierten Inhaber ihre Berechtigung generell nur indirekt, quasi über den Inhaber des elektronischen Wertpapiers, durch eine Depotbescheinigung zur Rechtsausübung gem. \6 Abs. 2 DepotG nachweisen (vgl. hierzu sogleich unter 4.). Unklar ist, warum diese allgemeinen Regelungen zum Rechtsnachweis im Rahmen des $\$ 29 \mathrm{eWpG}$ nicht ausreichen sollen, sondern (zusätzlich) die Glaubhaftmachung der eigenen Berechtigung erforderlich sein soll.

11 Vgl. Begründung RegE zu $\$ 3$ Abs. 1, BT-Drs. 19/26925, S. 41; vgl. aber auch die Begründung RegE zu $₫ 14$ Abs. 1 S. 1 Nr. 1, BT-Drs. 19/26925, S. 57, wonach mit der Inhaberschaft die Vermutung der formellen Berechtigung verknüpft ist.

12 Vgl. zur Begriffsbestimmung der Umtragung $\$ 4$ Abs. 8 eWpG.

13 Vgl. MüKo/Habersack, BGB, Bd.7 (8. Aufl. 2020), \$797 Rn.1.

14 Vgl. auch $\$ 19 \mathrm{eWpG}$, wonach der Inhaber einzeln eingetragener Kryptowertpapiere von der registerführenden Stelle verlangen kann, ihm zur Wahrnehmung seiner Rechte einen Registerauszug zur Verfügung zu stellen. 


\section{Vermutung für materielle Berechtigung des Inhabers?}

Nun ist zwar den $\$ \int 793 \mathrm{ff}$. BGB die Unterscheidung zwischen dem Inhaber, also dem mit tatsächlicher Sachherrschaft ausgestatteten Besitzer des Wertpapiers, ${ }^{15}$ und dem materiell Berechtigten der Sache nach nicht fremd (vgl. insbes. \$793 Abs. 1 S.1 BGB). ${ }^{16}$ Dennoch gibt es insofern gravierende Unterschiede zwischen Einzelurkunden bzw. elektronischen Wertpapieren in Einzeleintragung einerseits und sammeleingetragenen elektronischen Wertpapieren andererseits.

\section{a) Elektronische Wertpapiere in Einzeleintragung}

Ebenso wie die Inhaberschaft an einer (Einzel-)Urkunde die widerlegbare Vermutung für die materielle Berechtigung des Inhabers begründet (vgl. auch \$1006 BGB), ${ }^{17}$ findet sich auch für den Fall der Einzeleintragung elektronischer Wertpapiere in $\$ 27 \mathrm{eWpG}$ eine Vermutung für die materielle Berechtigung des Eingetragenen. Etwas seltsam ist bei dieser Regelung lediglich, dass nicht unmittelbar die Gläubigerstellung des eingetragenen Inhabers an der Forderung aus dem elektronischen Wertpapier, sondern die Eigentümerstellung an der bei elektronischen Wertpapieren nicht existenten Urkunde vermutet wird.

\section{b) Elektronische Wertpapiere in Sammeleintragung}

Anders ist dies aber im Fall der Sammeleintragung. Hier ist der eingetragene Inhaber gerade nicht der Inhaber der Forderung aus der Schuldverschreibung, sondern eine Wertpapiersammelbank bzw. ein Verwahrer ( $\int 8$ Abs.1 Nr.1 eWpG). Dabei fungiert die Wertpapiersammelbank zwar als gesetzlicher Ermächtigungstreuhänder ${ }^{18}$ der Berechtigten und zieht als solcher die Forderung beim Emittenten ein ( $\mathbb{\int} 3$ Abs. 1, 9 Abs. 1 S. 1, Abs. 2 eWpG). Daher hat hier die Eintragung zwar zur Folge, dass nur der eingetragene Inhaber gegenüber dem Emittenten formell legitimiert ist. Eine Vermutung zugunsten der materiellen Berechtigung des als Inhaber Eingetragenen resultiert hieraus gerade nicht; vielmehr stellt $\$ 9$ Abs. 2 S. 1 eWpG umgekehrt ausdrücklich fest, dass die Wertpapiersammelbank oder ein Verwahrer die Sammeleintragung treuhänderisch für die Berechtigten verwaltet, ohne selbst Berechtigter zu sein. Im Fall der

${ }^{15}$ Zum Begriff des Inhabers i. S.d. $\$ 793$ BGB Staudinger/Marburger, BGB (2015), $\$ 793$ Rn. 24; MüKo/Habersack, BGB, Bd.7 (8. Aufl. 2020), \$793 Rn. 29.

16 Vgl. MüKo/Habersack, BGB, Bd.7 (8. Aufl. 2020), \$793 Rn. $29 \mathrm{f}$.

17 Vgl. Hueck/Canaris, Recht der Wertpapiere (12. Aufl. 1986), \$24 I 2b; Zöllner, Wertpapierrecht (14. Aufl. 1987), $\$ 2$ II 1; Baumbach/Hefermehl/Casper, Wechselgesetz, Scheckgesetz, Recht des Zahlungsverkehrs (24. Aufl. 2020), WertpapierR Rn. 34; Staudinger/Marburger, BGB (2015), \$793 Rn. 23; MüKo/Habersack, BGB, Bd.7 (8. Aufl. 2020), Vor \$793 Rn. 16.

18 Vgl. Begründung RegE zu $₫ 9$ Abs. 1 und Abs. 2, BT-Drs. 19/26925, S. 50, wonach die Wertpapiersammelbank bzw. der Verwahrer lediglich Treuhänder der materiell Berechtigten sind, deren Vollrecht unberührt bleibt. 
Sammeleintragung fallen also formell Legitimierter und materiell Berechtigter (typischerweise) auseinander. Dies bedeutet eine Abweichung von dem Grundsatz des $\$ 793$ Abs. 1 S. 1 BGB, wonach die formelle Legitimation eine (widerlegbare) Vermutung für die materielle Berechtigung zur Folge hat (vgl. auch $\$ 1006$ BGB). Denn anders als die Inhaberstellung bei verbrieften Inhaberschuldverschreibungen gem. $\$ 793$ Abs. 1 S. 1 BGB begründet die Eintragung als Inhaber bei Sammeleintragungen nicht die Vermutung, der eingetragene Inhaber sei selbst Inhaber der Forderung aus der elektronischen Schuldverschreibung.

Dieses Konzept wurde teils recht scharf kritisiert; ${ }^{19}$ insbesondere wurde eingewandt, damit habe der Emittent je nach Lesart des $\$ 28$ Abs. 1 S. 1 eWpG doch nicht an den eingetragenen Inhaber zu leisten, weil dieser gerade nicht Berechtigter sei. ${ }^{20}$ In der Tat sind diese Regelungen auf den ersten Blick insofern etwas verwirrend, als der Berechtigte in $\$ 3$ Abs. 2 eWpG als der Inhaber des Rechts aus dem Wertpapier umschrieben ist, in $\$ 28$ Abs. 1 S. 1 eWpG mit der Berechtigung aber die Befugnis zur Einziehung der Forderung gemeint ist. Allerdings ist nicht nur der Rechtsinhaber berechtigt, eine Forderung gegenüber dem Schuldner geltend zu machen; forderungsberechtigt ist vielmehr auch derjenige, der gemäß bzw. analog $\ 185$ BGB oder kraft einer gesetzlich eingeräumten Ermächtigung berechtigt ist, die Forderung einzuziehen. So erklärt sich denn auch die Regelung des $₫ 28$ Abs. 1 S. 1 eWpG: Forderungsinhaber ist zwar der Rechtsinhaber i.S.d. $\$ 3$ Abs. 2 eWpG. Berechtigt, diese Forderung geltend zu machen, ist aber gem. $\$ 28$ Abs. 1 S. 1 eWpG grundsätzlich die im Wertpapierregister als Inhaber eingetragene und damit formell legitimierte Wertpapiersammelbank oder der eingetragene Verwahrer.

\section{Ausnabme: Feblende (Verfügungs-)Berechtigung des Inbabers}

Der im elektronischen Wertpapierregister eingetragene und daher formell legitimierte Inhaber ist aber - ähnlich wie bei verbrieften Schuldverschreibungen ausnahmsweise dann nicht (forderungs-)berechtigt, wenn ihm die Verfügungsbefugnis fehlt ( $\$ 28$ Abs. 1 S. $1 \mathrm{eWpG})$.

Dass der formell legitimierte Eingetragene gem. $\$ 28$ Abs. 1 S. 1 eWpG mangels Verfügungsbefugnis ausnahmsweise nicht forderungsberechtigt ist, kann bei Einzeleintragungen durchaus relevant werden. Denn hier ist für eine wirksame Übereignung eine dingliche Einigung zwischen dem Berechtigten und dem Erwerber sowie eine Umtragung des elektronischen Wertpapiers auf den Erwerber erforderlich, wobei diese Umtragung auf Weisung des Berechtigten

\footnotetext{
19 Vgl. etwa Stellungnahme des Deutschen Anwaltvereins zum RefE, S. 8 f.; Segna, Elektronische Wertpapiere im zentralen Register - Anmerkungen zum BMF-/BMJV-Referentenentwurf vom 10.8.2020 aus wertpapier- und depotrechtlicher Sicht-, WM 2020, 2301, $2309 \mathrm{f}$.

20 Vgl. Dubovitskaya, Gesetzentwurf zur Einführung von elektronischen Wertpapieren: ein zaghafter Schritt nach vorn, ZIP 2020, 2551, $2555 \mathrm{f}$.
} 
erfolgt sein muss (vgl. $\$ 25 \mathrm{eWpG}$ ). Hier ist durchaus denkbar, dass die dingliche Einigung unwirksam, die Umtragung aber erfolgt ist, so dass der eingetragene Inhaber nicht der materiell Berechtigte ist.

Im Fall der Sammeleintragung beruht hingegen die Verfügungsbefugnis der Wertpapiersammelbank bzw. der Verwahrer auf der gesetzlichen Vorschrift des \9 Abs. 2S. $1 \mathrm{eWpG}$, also einer gesetzlichen und nicht einer rechtsgeschäftlichen Ermächtigung der materiell Berechtigten. Daher dürfte bei sammeleingetragenen Wertpapieren der Fall einer fehlenden Verfügungsberechtigung des formell legitimierten Eingetragenen äußerst selten sein; denkbar ist dies allenfalls dann, wenn die Eintragung der Wertpapiersammelbank als formell legitimierte Inhaberin fehlerhaft und daher gegebenenfalls unwirksam war, etwa weil der zunächst einzeleingetragene Berechtigte keinen wirksamen Antrag gem. $\ 8$ Abs. 2 eWpG auf Sammeleintragung seiner Wertpapiere stellte.

Ist der formell Legitimierte nicht forderungsberechtigt, stellt sich zwar zunächst das Problem, dass dann weder der formell Legitimierte noch der materiell Berechtigte die Forderung aus dem elektronischen Wertpapier geltend machen kann. Dies lässt sich aber über einen Wertpapierregister-Berichtigungsanspruch desjenigen lösen, dessen Recht unzutreffend im Wertpapierregister verlautbart wird. Ein entsprechender Anspruch ließe sich auf $\$ 985$ BGB bzw. $\$ 1004$ BGB, jeweils i. V.m. $\$ 2$ Abs. 2, Abs. 3 eWpG, ggf. auch auf $\int 812$ Abs. 1 S. 1 BGB gründen. Je nach Sachverhaltsgestaltung sind auch verschuldensabhängige Ansprüche, wie etwa $\ 7$ Abs. 2 S. 2 eWpG bzw. $\ 823$ Abs. 1 BGB denkbar.

\section{Legitimation des Hinterlegers durch Depotbescheinigung}

Will der materiell Berechtigte selbst seinen Leistungsanspruch aus der Schuldverschreibung geltend machen, benötigt er hierfür eine Depotbescheinigung zur Rechtsausübung gem. \6 Abs. 2 DepotG. Die Vorlage der Urkunde, die bei Wertpapieren im Rechtssinne für die Geltendmachung des (verbrieften) Rechts grundsätzlich erforderlich ist, ${ }^{21}$ wird hier durch die Depotbescheinigung ersetzt. Der in der Depotbescheinigung Ausgewiesene gilt gem. $\$ 6$ Abs. 2 S. 3 DepotG zum Zwecke der Beweisführung als Inhaber des ihm zugewiesenen Anteils am Sammelbestand. Das Problem dieser Vorschrift besteht allerdings darin, dass die Depotbescheinigung - wie der Begriff auch nahelegt - dem Hinterleger ausgestellt wird; dieser kann allenfalls als Rechtsinhaber vermutet werden, muss aber nicht (zwangsläufig) der Rechtsinhaber sein. Insbesondere gibt es bei Verwahrketten auf jeder Stufe der Kette (zumindest) einen Hinterleger. Geht man vom Wortlaut des $\$ 6$ Abs. 2 DepotG aus, müssten eigentlich alle Hinterleger auf jeder Stufe der Kette zum Zwecke der Beweisführung als In-

${ }^{21}$ Heute ganz h.M., vgl. statt vieler Hueck/Canaris, Recht der Wertpapiere (12. Aufl. 1986), \$1 II 3b); Zöllner, Wertpapierrecht (14. Aufl. 1987), \$3 III 4b); MüKo/Habersack, BGB, Bd. 7 (8. Aufl. 2020), Vor $\$ 793$ Rn. $10 f$. 
haber gelten. Dieses Problem ließe sich zwar eventuell noch durch eine entsprechende Anwendung der $\$ \$ \int 123$ Abs. 4 S. 1, 67c Abs. 3 AktG lösen; danach würde also nur der Letztintermediär seinem Hinterleger eine Depotbescheinigung zur Rechtsausübung ausstellen. Das gravierendere Problem, nämlich die Frage nach dem Verhältnis der Rechte der Hinterleger und eventueller Rechte der materiell Berechtigten, ist damit aber noch nicht gelöst. Auch die Gesetzesbegründung zu $₫ 6$ Abs. 2 DepotG schafft hinsichtlich dieses Verhältnisses keine Klarheit; vielmehr spricht die Begründung des Regierungsentwurfs bezogen auf die Depotbescheinigung einmal mehr von einer gesetzlichen Fiktion, und zwar einer gesetzlichen Fiktion der Inhaberschaft zu Beweiszwecken. ${ }^{22}$ Anders als die Inhaberschaft an der Urkunde ( $\mathbb{5} 793$ Abs. 1 S.1 BGB) begründet die Depotbescheinigung zur Rechtsausübung aber (wohl) nicht nur eine (bloße) Vermutung für die materielle Berechtigung des in der Depotbescheinigung ausgewiesenen Inhabers. Denn immerhin hat der Verwahrer für die Richtigkeit der ausgestellten Depotbescheinigung einzustehen ( $\$ 6$ Abs. 2 S. 2 DepotG); auch soll - so die Gesetzesbegründung - diese Pflicht des Verwahrers drittschützende Wirkung zugunsten des Ausstellers entfalten. ${ }^{23}$ Da der Aussteller durch die Depotbescheinigung vor einer mehrfachen Inanspruchnahme geschützt werden soll, kann sich deren rechtliche Bedeutung kaum in einer bloßen Vermutung für die materielle Berechtigung des in der Depotbescheinigung ausgewiesenen Inhabers erschöpfen. Die Depotbescheinigung wird zwar dem Hinterleger ausgestellt, soll aber offenbar die Wirkung haben, diesen als materiell Berechtigten auszuweisen. Umso gravierender ist, dass der Gesetzgeber zur Frage, ob und wie die materiell Berechtigten ihre Rechte ausüben können, nicht Stellung bezogen hat.

Das Gesetz zur Einführung von elektronischen Wertpapieren scheint allerdings entweder von der Identität des schuldrechtlich und des dinglich Berechtigten oder aber davon auszugehen, dass der schuldrechtlich berechtigte Hinterleger von dem dinglich am Sammelbestand Berechtigten zur Rechtsausübung ermächtigt wird. Hierfür spricht jedenfalls $\$ 6$ Abs. 2 S. 4 DepotG; denn danach ist vorgesehen, dass der Hinterleger bei Geltendmachung eines Leistungsanspruchs gegen den Emittenten seinen Anteil am Sammelbestand an den Aussteller überträgt. Dies setzt aber voraus, dass der Hinterleger entweder selbst der Rechtsinhaber ist oder von diesem zur Rechtsübertragung ermächtigt wurde.

22 Vgl. Begründung zu $\$ 6$ Abs. 2 RegE-DepotG, BT-Drs. 19/26925, S. 72; vgl. aber auch Beschlussempfehlung und Bericht des Finanzausschusses zum RegE BT-Drs. 19/29372, S. 60: Danach handelt es sich bei dem Hinterleger in der Regel (aber eben nur in der Regel, angefügt v. Verf.) um den Berechtigten.

23 Vgl. Begründung zu \$6 Abs. 2 RegE-DepotG, BT-Drs. 19/26925, S. 72. 


\section{Liberationsfunktion der Registereintragung zugunsten des Emittenten}

Die Liberationswirkung der Eintragung gem. $\$ 28$ Abs. 1 S. 2 eWpG entspricht zunächst der Regelung des $\$ 793$ Abs. 1 S. 2 BGB für verbriefte Inhaberschuldverschreibungen: So wird der Emittent elektronischer Wertpapiere von seiner Leistungspflicht auch dann befreit, wenn er an den im elektronischen Wertpapierregister als Inhaber Eingetragenen leistet. Dies gilt gerade auch dann, wenn es sich hierbei - wie im Fall von Sammeleintragungen - nicht um den materiell berechtigten Forderungsinhaber handelt.

Allerdings wird $\$ 793$ Abs. 1 S. 2 BGB nach h. M. restriktiv interpretiert. Danach besteht bei verbrieften Inhaberschuldverschreibungen die Liberationswirkung des $\ 793$ Abs. 1 S. 2 BGB nicht, wenn der Aussteller die fehlende Berechtigung des Inhabers der Urkunde positiv kennt und unschwer nachweisen kann. ${ }^{24}$ Zumeist wird angenommen, lediglich grob fahrlässige Unkenntnis des Ausstellers von der fehlenden Berechtigung des Inhabers der Urkunde schließe analog Art. 40 Abs. 3 WG die Liberationswirkung ebenfalls aus. ${ }^{25}$ Inhaltlich soll nach h. M. auch der gute Glaube an die Verfügungsbefugnis, die Vertretungsmacht sowie die Geschäftsfähigkeit des Inhabers geschützt werden. ${ }^{26}$

Bei elektronischen Wertpapieren stellt sich nun die Frage, ob $\$ 28$ Abs. 1 S. 2 eWp in entsprechender Weise wie $\$ 793$ Abs. 1 S. 2 BGB restriktiv interpretiert werden sollte. Im Ergebnis wird dies wohl zu bejahen sein, zumal gem. $\$ 2$ Abs. 2 eWpG ein elektronisches Wertpapier - vorbehaltlich spezieller Regelungen des eWpG - dieselbe Rechtswirkung wie ein Wertpapier entfaltet, das mittels Urkunde begeben worden ist. Allerdings dürfte diese einschränkende Interpretation des $\$ 28$ Abs. 1 S. 2 eWpG nicht von allzu großer praktischer Bedeutung sein. Dies gilt insbesondere im Fall sammeleingetragener elektronischer Wertpapiere. Denn hier ist der eingetragene Inhaber - wenngleich nicht selbst Berechtigter als gesetzlicher Ermächtigungstreuhänder über die elektronischen Wertpapiere verfügungsbefugt ( $\$ 9$ Abs. 2 S. $1 \mathrm{eWpG})$.

${ }^{24}$ Vgl. BeckOGK/Vogel, BGB (01.07.2021), \$793 Rn. 158; MüKo/Habersack, BGB, Bd. 7 (8. Aufl. 2020), \$793 Rn. 42; Hueck/Canaris, Recht der Wertpapiere (12. Aufl. 1986), \$24 III 3; Zöllner, Wertpapierrecht (14. Aufl. 1987), \$27 I 3.

${ }_{25} \mathrm{Vgl}$. Hueck/Canaris, Recht der Wertpapiere (12. Aufl. 1986), \$24 III 3; Zöllner, Wertpapierrecht (14. Aufl. 1987), \$27 I 3; MüKo/Habersack, BGB, Bd. 7 (8. Aufl. 2020), \$793 Rn. 43; BeckOGK/Vogel, BGB (01.07.2021), \$793 Rn.159.

${ }^{26} \mathrm{Vgl}$. MüKo/Habersack, BGB, Bd.7 (8. Aufl. 2020), \$793 Rn. 42 f.; BeckOGK/Vogel, BGB, (01.07.2021), \$793 Rn. 157; Zöllner, Wertpapierrecht(14. Aufl.1987), \$27 I3; a. A. Hueck/ Canaris, Recht der Wertpapiere (12. Aufl. 1986), \$24 III 3. 
V. Einwendungen des Emittenten und Einwendungsausschluss

\section{Einwendungen}

Ferner stellt sich die Frage, welche Einwendungen der Emittent der elektronischen Schuldverschreibung geltend machen kann, wenn er auf Leistung in Anspruch genommen wird. Insoweit intendiert $\$ 28 \mathrm{Abs.} 2 \mathrm{eWpG}$, die Regelung des $\$ 796$ BGB auf elektronische Schuldverschreibungen zu übertragen. ${ }^{27}$ Dies gelingt aber nicht vollständig.

Zunächst lassen sich die Einwendungen, die sich aus der Eintragung ( $\$ 28$ Abs. 2 Nr. 1 eWpG) sowie aus den Anleihebedingungen ( $\$ 28$ Abs. 2 Nr.3 eWpG) ergeben, quasi als urkundliche Einwendungen bezeichnen..$^{28}$ Solche Einwendungen, die der Aussteller verbriefter Inhaberschuldverschreibungen gem. $\$ 796$ Alt. 2 BGB hat, kann also auch der Emittent elektronischer Wertpapiere erheben, wenn er aus dem Wertpapier in Anspruch genommen wird. Hinsichtlich $₫ 28$ Abs. 2 Nr. 1 und $3 \mathrm{eWpG}$ gelingt daher eine $\$ 796$ Alt. 2 BGB entsprechende Regelung.

Problematischer ist aber schon $\$ 28$ Abs. 2 Nr. 2 eWpG, wonach der Emittent Einwendungen geltend machen kann, die die Gültigkeit der Eintragung betreffen. Zwar erfasst diese Regelung - ebenso wie $\$ 796$ Alt. 1 BGB - Mängel der Erklärung des Emittenten im Rahmen der Ausgabe der Wertpapiere. Hinsichtlich sonstiger Mängel des Begebungsvertrags zeigt sich aber wiederum, dass der Regelungsgehalt dieser Vorschrift im Fall der Sammeleintragung und der Einzeleintragung unterschiedlich ist.

So ist im Fall der Einzeleintragung der erste eingetragene Inhaber regelmäßig auch der Ersterwerber der Schuldverschreibung und daher der Vertragspartner des Emittenten bei Abschluss des Begebungsvertrags. Können vom Emittenten Einwendungen betreffend die Gültigkeit der Eintragung geltend gemacht werden, sind damit (auch sonstige) Mängel des Begebungsvertrags erfasst. Etwas anders ist dies aber bei sammeleingetragenen Schuldverschreibungen. Da hier der Ersterwerber der Wertpapiere gerade nicht in das Wertpapierregister eingetragen wird, kann der Emittent - anders als bei $\$ 796$ Alt. 1 BGB $^{29}$ - über $\$ 28$ Abs. 2 Nr. 2 eWpG Mängel des Begebungsvertrags zumindest nicht umfassend geltend machen. Daher entspricht $\$ 28$ Abs. 2 Nr. 2 eWpG zumindest nicht vollständig der Regelung des $\$ 796$ Alt. 1 BGB.

27 Vgl. auch die Begründung RegE zu $\$ 28$ Abs. 2, BT-Drs. 19/26925, S. 68.

28 Vgl. auch dazu, dass die Eintragung eines elektronischen Wertpapiers unter eindeutiger und unmittelbar erkennbarer Bezugnahme auf die niedergelegten Emissionsbedingungen erfolgt, $\mathbb{\$} 4$ Abs. 4, Abs. 7, $\$ 5$ Abs. 1 eWpG sowie die Begründung zu $\ 4$ Abs. 4, BT-Drs. 19/26925, S. $42 \mathrm{f}$.

${ }^{29}$ Vgl. Staudinger/Marburger, BGB (2015), $\$ 796$ Rn. 5; MüKo/Habersack, BGB, Bd.7 (8. Aufl. 2020), \$796 Rn.9. 
Die Regelung des $\$ 28$ Abs. 2 Nr. 4 eWpG wurde indes im Vergleich zum Regierungsentwurf ${ }^{30}$ insofern verbessert, als das Gesetz nunmehr den Fall der Einzeleintragung ( $\$ 28$ Abs. 2 Nr.4a) eWpG) und den der Sammeleintragung ( $(28$ Abs. 2 Nr. 4b) eWpG) unterscheidet. Im Fall der Einzeleintragung kann der Emittent elektronischer Schuldverschreibungen nur Einwendungen erheben, die ihm unmittelbar gegen den Inhaber zustehen. Vom Wortlaut her gesehen entspricht diese Regelung $\$ 796$ Alt. 3 BGB. Solche unmittelbaren Einwendungen sind nach ganz h. M. zunächst solche Einwendungen, die sich aus einem besonderen persönlichen Rechtsverhältnis zwischen dem Aussteller und einem bestimmten Inhaber ergeben. ${ }^{31}$ Diese Vorschrift erscheint bei Einzeleintragungen durchaus passend. Denn hier entspricht der eingetragene Inhaber elektronischer Wertpapiere dem Inhaber verbriefter Schuldverschreibungen.

Anders ist dies aber bei sammeleingetragenen elektronischen Wertpapieren. Denn in diesem Fall sind zwar die Wertpapiersammelbank bzw. der Verwahrer die im Wertpapierregister eingetragenen Inhaber und damit die formell Legitimierten; sie sind aber nicht die Berechtigten. Aufgrund der in das Gesetz aufgenommenen Regelung des $\ 28$ Abs. 2 Nr. 4b) eWpG kann der Emittent elektronischer Schuldverschreibungen im Fall einer Sammeleintragung nur solche Einwendungen erheben, die ihm unmittelbar gegen denjenigen zustehen, der auf Grund einer Depotbescheinigung zur Rechtsausübung gem. $\$ 6$ Abs. 2 S. 3 DepotG als Inhaber gilt. Dies stellt insofern eine Verbesserung gegenüber dem Regierungsentwurf dar, als nach dem jetzigen Gesetzeswortlaut der Emittent nicht nur Einwendungen gegen den im Wertpapierregister eingetragenen gesetzlichen Ermächtigungstreuhänder ${ }^{32}$ geltend machen kann. Damit wurde auch eine - wenngleich nicht vollständige - Angleichung an die Parallelvorschrift ${ }^{33}$ des $\$ 6$ Abs. 6 BSchuWG erreicht; denn danach kann der Schuldner der Sammelschuldbuchforderung nur solche Einwendungen erheben, die ihm unmittelbar gegen den Gläubiger (und gerade nicht gegen die Wertpapiersammelbank) zustehen. Vergleicht man $\$ 28$ Abs. 2 Nr. 4b) eWpG mit der Parallelvorschrift des $₫ 6$ Abs. 6 BSchuWG, bleibt allerdings problematisch, dass im Rahmen des $\ 28$ Abs. 2 Nr. 4b) eWpG der Emittent nur solche Einwendungen

30 BT-Drs. 19/26925, S. 19.

31 Vgl. Staudinger/Marburger, BGB (2015), §796 Rn. 9; MüKo/Habersack, BGB, Bd.7 (8. Aufl. 2020), \$796 Rn. 13; BeckOK/Gebrlein, BGB (58. Ed. 01.05.2021), \$796 Rn. 4; BeckOGK/Vogel, BGB (01.07.2021), \$796 Rn. 18.

32 Vgl. Begründung RegE zu $\int 9$ Abs. 1 und Abs.2, BT-Drs. 19/26925, S. 50, wonach die Wertpapiersammelbank bzw. der Verwahrer lediglich Treuhänder der materiell Berechtigten sind, deren Vollrecht unberührt bleibt.

33 Vgl. dazu, dass der RegE in Anlehnung an das BSchuWG konzipiert wurde, Begründung RegE A. Allgemeiner Teil II. Wesentlicher Inhalt, BT-Drs. 19/26925, S. 29; Begründung RegE zu $₫ 8$ Abs. 1 Nr. 1, BT-Drs. 19/26925, S. 49, zu \$8 Abs. 2, BT-Drs. 19/26925, S. 50 sowie zu $₫ 9$ Abs. 1 und 2, BT-Drs. 19/26925, S. 50. 
geltend machen kann, die ihm unmittelbar gegen den schuldrechtlich berechtigten Hinterleger zustehen (vgl. zur Depotbescheinigung zur Rechtsausübung $\$ 6$ Abs. 2 Depot $^{34}$ ). Nach dem Wortlaut des Gesetzes dürfte der Emittent hingegen Einwendungen unmittelbar gegen den dinglich am Sammelbestand Berechtigten nicht geltend machen können.

Daneben stellt aber auch die Einwendung, der Inhaber sei nicht verfügungsbefugt, eine zwar nicht persönliche, aber ebenfalls eine unmittelbare Einwendung des Emittenten dar. ${ }^{35}$ Die Einwendung mangelnder Verfügungsbefugnis des im Wertpapierregister Eingetragenen kann im Fall der Einzeleintragung durchaus relevant werden; dass der Emittent diese Einwendung geltend machen kann, ergibt sich indes bereits aus $\$ 28$ Abs. 1 S. 1 eWpG. Hingegen ist im Fall der Sammeleintragung die Wertpapiersammelbank bzw. der Verwahrer gem. $\$ 9$ Abs. 2 S. 1 eWpG verfügungsbefugt. Hier besteht die Verfügungsbefugnis des eingetragenen Inhabers also unabhängig davon, welche konkrete Person materiell Berechtigter ist. Daher dürfte bei sammeleingetragenen Wertpapieren die Einwendung mangelnder Verfügungsbefugnis des im Wertpapierregister eingetragenen Verwahrers ( $\$ 28$ Abs. 1 S. $1 \mathrm{eWpG}$ ) kaum relevant werden. Soweit es allerdings um den mit einer Depotbescheinigung zur Rechtsausübung ausgestatteten Hinterleger geht, kann der Emittent die Einwendung, dieser sei nicht verfügungsbefugt, gegebenenfalls gem. $\$ 28$ Abs. 2 Nr. 4b) eWpG erheben.

\section{Einwendungsausschluss}

Die Frage, unter welchen Voraussetzungen Einwendungen des Emittenten ausgeschlossen sind, wird im eWpG nicht ausdrücklich geregelt. Allerdings ist davon auszugehen, dass insoweit ebenso wie für verbriefte Wertpapiere (vgl. $\$ 2$ Abs. 2 eWpG) die allgemeinen Grundsätze (der Rechtsscheinhaftung) gelten: Danach werden urkundliche Einwendungen des Emittenten - da aus der Urkunde ersichtlich - durch Verkehrsgeschäfte nicht ausgeschlossen. Bezogen auf elektronische Wertpapiere müsste dies auch für Einwendungen gelten, die sich aus der Eintragung ergeben ( $\$ 4 \mathrm{Abs} .4 \mathrm{eWpG}$ ). Hingegen werden Mängel betreffend die Gültigkeit der Emission grundsätzlich bei Gutgläubigkeit des (Zweit-)Erwerbers ausgeschlossen; dabei liegt Gutgläubigkeit des Erwerbers dann vor, wenn dieser den Mangel weder kennt noch grob fahrlässig nicht kennt. Sofern allerdings die Mängel hinsichtlich der Gültigkeit der Emission die Zurechenbarkeit des Rechtsschein ausschließen, sind diese Mängel nicht präkludierbar. Hingegen werden unmittelbare persönliche Einwendungen anaIII.4.

${ }^{34}$ Vgl. aber auch zu den durch die Depotbescheinigung aufgeworfenen Problemen oben

35 Vgl. Staudinger/Marburger, BGB (2015), \$796 Rn. 9; MüKo/Habersack, BGB, Bd.7 (8. Aufl. 2020), $\$ 796$ Rn. 5, 15. 
log Art. 17 WG, Art. 22 ScheckG ausgeschlossen, sofern nicht der betreffende Inhaber zum Nachteil des Emittenten gehandelt hat. ${ }^{36}$

\section{Verkehrsschutzfunktion durch gutgläubigen Erwerb}

Eine wesentliche Funktion der Verbriefung von Forderungen in Inhaberschuldverschreibungen besteht darin, gutgläubigen Erwerb zu ermöglichen und so die Verkehrsfähigkeit dieser Forderungen zu steigern. Hier stellt sich nun die Frage, ob und ggf. wie dieses Ziel bei elektronischen Wertpapieren erreicht wird. Dabei ist zwischen elektronischen Wertpapieren in Sammeleintragung und solchen in Einzeleintragung zu unterscheiden.

\section{Elektronische Wertpapiere in Sammeleintragung}

Da elektronische Wertpapiere in Sammeleintragung als Wertpapiersammelbestand gelten ( $\$ 9$ Abs. 1 S. $1 \mathrm{eWpG}$ ), finden auf die Übertragung der Miteigentumsanteile der Berechtigten an diesem Sammelbestand vorrangig die $\$ \int 929 \mathrm{ff}$. BGB und subsidiär $\$ 24$ Abs. 2 DepotG Anwendung. Daher wirft die Übereignung dieser Miteigentumsanteile dieselben Probleme wie die Übereignung der Miteigentumsanteile verbriefter sammelverwahrter Wertpapierurkunden auf. Dies betrifft zum einen die Frage, wer bei der regelmäßigen Einschaltung von Intermediären Partei der dinglichen Einigung ist. ${ }^{37} \mathrm{Im}$ vorliegenden Zusammenhang wesentlicher ist aber, ob hier eine Übergabe bzw. Übergabesurrogat gegeben ist bzw. durch welches sonstige Publizitätselement ersetzt wird.

In diesem Zusammenhang könnte $\$ 2$ Abs. 3 eWpG von Bedeutung sein, wonach ein elektronisches Wertpapier als Sache gilt. Ist wie bei elektronischen Wertpapieren keine Sache vorhanden, ist jedoch nicht gerade klar ersichtlich, wer der unmittelbare oder mittelbare Besitzer erster, zweiter oder dritter Stufe der fingierten Sache ist. Soweit es allerdings um Verfügungen über sammeleingetragene elektronische Wertpapiere geht, können diese grundsätzlichen Anwendungsprobleme der Gleichstellungsfiktion dahingestellt bleiben. Denn sammeleingetragene elektronische Wertpapiere sollen wie verbriefte, sammelverwahrte Urkunden übertragen werden. Wie bereits an anderer Stelle ausführlich erörtert, können indes die dinglich Berechtigten auch bei sammelverwahrten verbrieften Urkunden nicht mehr als deren Besitzer angesehen werden. Besonders deutlich wird dies bei Dauerglobalurkunden, da die Anleger hier

36 Vgl. zu diesen Grundsätzen Hueck/Canaris, Recht der Wertpapiere (12. Aufl. 1986), \$24 III 2; Zöllner, Wertpapierrecht (14. Aufl. 1987), \$27 I 4; MüKo/Habersack, BGB, Bd. 7 (8. Aufl. 2020), $\$ 796$ Rn.3f.; Staudinger/Marburger, BGB (2015), \$796 Rn. 2-12.

37 Vgl. hierzu etwa Einsele, Bank- und Kapitalmarktrecht - Nationale und Internationale Bankgeschäfte (4. Aufl. 2018), $\$ 9$ Rn. 23-27 m. w. N. 
keinen Anspruch auf Auslieferung von Einzelurkunden und daher auch keinen mittelbaren Mitbesitz an dem Sammelbestand haben (vgl. \$\$ 868, 870 BGB). Dies gilt indes auch für sonstige sammelverwahrte Urkunden, da die $\mathbb{S} \mathbb{S} 7,8 \mathrm{De}-$ potG lediglich schuldrechtliche Ansprüche auf Auslieferung einer Sache aus einer Gesamtheit an Sachen nach Wahl des Schuldners, nicht aber eine Sachherrschaft des Anlegers an der Sachgesamtheit begründen. ${ }^{38}$

Auch die h. M., die im Grundsatz (mittelbaren Mit-)Besitz der Anleger bejaht, erkennt andererseits, dass sich damit die Möglichkeit gutgläubigen Erwerbs kaum begründen lässt. Denn die Anleger disponieren auch dann lediglich über ihren Mitbesitz an dem Sammelbestand. Mitbesitz begründet aber hinsichtlich der Höhe des Miteigentumsanteils keinen aussagekräftigen tauglichen Rechtsscheinträger. ${ }^{39}$ Daher wird für den Effektengiroverkehr heute überwiegend vertreten, die Buchung im Verwahrungsbuch fungiere entweder anstelle des Mitbesitzes $^{40}$ oder neben dem Mitbesitz ${ }^{41}$ als Rechtsscheinträger. Dies ist jedoch abzulehnen. Denn im Bereich des Sachenrechts muss ein Rechtsscheinträger für den Rechtsverkehr offenkundig sein. Buchungen weisen jedoch bereits aufgrund des Bankgeheimnisses grundsätzlich nicht das Kriterium allgemeiner Offenkundigkeit auf.

38 Vgl. hierzu Einsele, Bank- und Kapitalmarktrecht - Nationale und Internationale Bankgeschäfte (4. Aufl. 2018), \$9 Rn. 17-19.

39 Einsele, Wertpapierrecht als Schuldrecht - Funktionsverlust von Effektenurkunden im internationalen Rechtsverkehr (1995), S. 105 ff.; MüKo/Einsele, HGB, Bd. 6 (4. Aufl. 2019), Q. Depotgeschäft Rn.117.

40 Vgl. Canaris, Bankvertragsrecht (2. Aufl. 1981), Rn. 2027; Hueck/Canaris, Recht der Wertpapiere (12. Aufl. 1986), \$1 III 1c; Heinsius/Horn/Than, DepotG (1975), \$6 Rn. 91; Fabricius, Zur Theorie des stückelosen Effektengiroverkehrs mit Wertrechten aus Staatsanleihen: Zugleich ein Beitrag zur Frage der Abgrenzung von Schuldrecht und Sachenrecht, AcP 162 (1963), 456, 481 f.; Lütticke, Elektronische Verbriefung von Effektenrechten? (1980), S. 165; K. Peters, Rechtliche Entwicklungsmöglichkeiten im Effektenbereich (1983), S. 31; Wolter, Effektenkommission und Eigentumserwerb, zugleich ein Beitrag zur Lehre vom Geschäft für denjenigen, den es angeht (1979), S.306; Körner, Die Entstückung des Effektenwesens nach dem Rechtsmodell der Sammelschuldbuchforderung (1971), S 117; Brink, Rechtsbeziehungen und Rechtsübertragung im nationalen und internationalen Effektengiroverkehr (1976), S. 102; H.P. Westermann, Das Girosammeldepot im deutschen Recht, RabelsZ 49 (1985), 214, 231; Walz, Sachenrecht für Nicht-Sachen?: Kritik einer Systemanalogie, KrVjschr 1986, 131 (138f.), insbes. Fn. 54, 161 f.; ähnlich, nämlich Buchungsverschaffungsmacht als Rechtsscheinträger, Schwarz, Globaler Effektenhandel (2016), S. 375 ff.; a. A. allerdings Becker, Das Problem des gutgläubigen Erwerbs im Effektengiroverkehr (1981), S. 30ff., insbes. S. 42, wonach eine Rechtsfortbildung mit der Buchung als Rechtsscheinträger nicht erforderlich ist, $\mathrm{da}$ gutgläubiger Erwerb auch ohne Rechtsfortbildung gem. den $\$ \mathbb{S} 929 \mathrm{ff} ., 932 \mathrm{ff}$. BGB möglich sei.

${ }^{41}$ Insoweit sehr deutlich Koller, Der gutgläubige Erwerb von Sammeldepotanteilen an Wertpapieren im Effektengiroverkehr, DB 1972, 1904, 1909: „Der gutgläubige Erwerb von Sammeldepotanteilen beruht auf dem mittelbaren Besitz des Verfügenden und der im Depotbuch der Wertpapiersammelbank ausgewiesenen Miteigentumsquote“; wohl ebenso MüKo/K. Schmidt, BGB, Bd.7 (8. Aufl. 2020), \$747 Rn.22; Wust, Die grenzüberschreitende Verbuchung von Wertpapieren, Möglichkeiten und Grenzen moderner Kollisionsnormen im Hinblick auf unterschiedliche materiellrechtliche Berechtigungen an Wertpapieren (2011), S. $152 \mathrm{ff}$. 
Im Bereich der elektronischen Wertpapiere könnte indes der Registereintragung diese allgemeine Publizitätswirkung zukommen. Bezogen auf Sammeleintragungen ist aber zu beachten, dass die Eintragungen von vornherein nur die formell berechtigte Wertpapiersammelbank bzw. den formell berechtigten Verwahrer verlautbaren, wobei diese Verwahrer gerade nicht die materiell Berechtigten sind. Auch findet bei Verfügungen über sammeleingetragene elektronische Wertpapiere gerade keine Umtragung im Wertpapierregister statt; sammeleingetragene elektronische Wertpapiere werden vielmehr ebenso wie sonstige Wertpapiere im Sammelbestand ${ }^{42}$ nicht durch Registereintragungen, sondern regelmäßig zumindest faktisch durch Umbuchungen, häufig durch Umbuchungen der Wertpapiersammelbank, übertragen. Insofern werden durch den Gesetzentwurf zwar keine bestehenden Probleme gelöst, aber auch keine neuen Probleme geschaffen.

\section{Elektronische Wertpapiere in Einzeleintragung}

Für Verfügungen über elektronische Wertpapiere in Einzeleintragung gelten hingegen die Sondervorschriften der $\$ \$ 24 \mathrm{ff}$. eWpG. Danach wird das Eigentum an einem elektronischen Wertpapier durch dingliche Einigung und Umtragung übertragen ( $\$ 25$ Abs. $1 \mathrm{eWpG}$ ); hierzu wird der bisherige Inhaber des elektronischen Wertpapiers durch den neuen Inhaber ersetzt ( $\$ 4$ Abs. 8 eWpG). Dabei hat die Umtragung auf Weisung des Berechtigten zu erfolgen, wobei hier - anders als bei sammeleingetragenen elektronischen Wertpapieren - der Inhaber als Berechtigter gilt, es sei denn, dass dem Erwerber zum Zeitpunkt seiner Eintragung etwas anderes bekannt oder infolge grober Fahrlässigkeit unbekannt ist $(\mathbb{2} 26 \mathrm{eWpG})$. An die Umtragung des elektronischen Wertpapiers knüpft sich auch die Möglichkeit gutgläubigen Erwerbs an, wobei die Gutgläubigkeit des Erwerbers vermutet wird. Ist dem Erwerber im Zeitpunkt seiner Eintragung also weder bekannt noch grob fahrlässig unbekannt, dass der eingetragene Inhaber nicht der Berechtigte ist, kann er das elektronische Wertpapier gutgläubig erwerben $(\$ 26 \mathrm{eWpG})$.

Dies setzt indes voraus, dass der Erwerber in das Wertpapierregister zumindest Einsicht nehmen kann, wenngleich nicht unbedingt muss. ${ }^{43}$ Zunächst hat zwar jeder Teilnehmer des Registers - ohne die Notwendigkeit der Darlegung eines berechtigten Interesses - das Recht zur Registereinsicht ( $\$ 10$ Abs. 1 eWpG). Laut der Begründung zum RegE gehört zum Teilnehmerkreis etwa der Inhaber, der Berechtigte, aber auch der Betroffene, z. B. der Emittent. ${ }^{44}$ Ein nur potenzieller Erwerber gehört jedoch nicht dazu, zumal das Einsichtnahmerecht der Teilnehmer nur im Hinblick auf ihre jeweilige Funktion oder Position im

\footnotetext{
${ }^{42}$ Vgl. auch die Begründung RegE zu $\$ 9$ Abs. 1 und Abs. 2, BT-Drs. 19/26925, S. 50.

43 Vgl. auch die Begründung RegE zu \$26, BT-Drs. 19/26925, S. 67.

44 Vgl. die Begründung RegE zu $\$ 10$ Abs. 1, BT-Drs. 19/26925, S. 51.
} 
Register bestehen soll. Da potenzielle Erwerber aber gerade noch keine Funktion oder Position im Register haben, lässt sich für diese Personengruppe jedenfalls aus $\ 10$ Abs. $1 \mathrm{eWpG}$ kein Einsichtnahmerecht begründen. Ein potenzieller Erwerber hat hingegen gem. $\$ 10$ Abs. 2 eWpG grundsätzlich ein Recht zur Einsicht in das Wertpapierregister, sofern er hieran ein berechtigtes Interesse darlegt. Ausweislich der Begründung zum RegE gehören gerade auch potenzielle Erwerber zu dem Personenkreis, der bei berechtigtem Interesse ein Recht zur Einsichtnahme hat. ${ }^{45}$ Damit besteht auch für Dritte die Möglichkeit der Einsichtnahme in das Wertpapierregister.

Aus dem Wertpapierregister ergibt sich zunächst aber nur der Inhaber des Wertpapiers und gerade nicht die Person des (materiell berechtigten) Rechtsinhabers. Hiergegen lässt sich jedoch einwenden, dass im vorliegend maßgeblichen Fall der Einzeleintragung der Inhaber ja zugleich die Person ist, die das Wertpapier als Berechtigte hält ( $\$ 8$ Abs. 1 Nr. 2 eWpG); die Tatsache, dass es sich um eine Einzeleintragung handelt, ist aber ebenfalls aus dem Wertpapierregister ersichtlich ( $\int \mathbb{S} 13$ Abs. 1 Nr.5, 17 Abs. 1 Nr. 5 eWpG). Auch wurde im Gesetzgebungsverfahren die Definition des einzeleingetragenen Inhabers als „Person, die das elektronische Wertpapier für sich selbst hält" (so noch $\ 8$ Abs. 1 Nr. 2 RegE) zu Recht geändert. Irritierend war die Definition des RegE, da sie die Frage aufwarf, ob ein fiduziarischer Treuhänder im Fall der Einzeleintragung möglicherweise deshalb nicht im Wertpapierregister eingetragen werden kann, weil er das Wertpapier nicht für sich selbst, sondern für den Treugeber hält. Allerdings ging bereits die Begründung zu $\$ 8$ Abs. 1 Nr. 2 RegE davon aus, bei der Einzeleintragung werde der Berechtigte, also derjenige, der das Recht aus dem Wertpapier innehat ( $\ 3$ Abs. 2 RegE und eWpG), mithin der Rechtsinhaber der elektronisch „verkörperten“ Forderung, in das Wertpapierregister eingetragen. ${ }^{46}$ Daher lässt sich bei Einzeleintragungen aus dem Wertpapierregister tatsächlich auch die Person des Berechtigten, wenn auch nicht unbedingt dessen namentliche Identität, entnehmen. Denn die Bezeichnung des Inhabers kann im Fall von Einzeleintragungen in ein zentrales Wertpapierregister auch durch Zuordnung einer eindeutigen Kennung erfolgen ( $\$ 13$ Abs. 1 Nr.6, Abs. 2 S. 2 eWpG), während die Zuordnung einer eindeutigen Kennung bei Kryptowertpapierregistern sogar zwingend vorgeschrieben ist ( $\mathbb{1} 17$ Abs. 1 Nr. 6, Abs. 2 S. 2 eWpG). Daher lässt sich aus dem Wertpapierregister nicht unbedingt die namentliche Identität des Inhabers entnehmen. Immerhin bietet das Recht zur Einsichtnahme in das Wertpapierregister aber die Möglichkeit eines Abgleichs zwischen der Person des Veräußerers und der des Eingetragenen, wenngleich gegebenenfalls nur durch einen Abgleich der Kennungen. Das Recht zur Einsichtnahme in das elektronische Wertpapierregister gem. $\$ 10$ Abs. $2 \mathrm{eWpG}$ um-

45 Vgl. die Begründung RegE zu $\ 10$ Abs. 2, BT-Drs. 19/26925, S. 52.

46 Vgl. die Begründung RegE zu \$8 Abs. 1 Nr. 2, BT-Drs. 19/26925, S. 50. 
fasst hingegen nicht auch einen Anspruch auf Auskunft über die Identität und Adresse eines Inhabers.

Ein Auskunftsrecht hinsichtlich der Identität und Adresse eines Inhabers besteht nur unter den sehr engen Voraussetzungen des $\$ 10$ Abs. 3 eWpG. Hierfür ist indes nicht nur ein berechtigtes, sondern darüber hinaus ein besonderes berechtigtes Interesse des Auskunftsuchenden erforderlich. Laut der Gesetzesbegründung ist insoweit eine Abwägung mit den Interessen der betroffenen Personen erforderlich, wobei ein besonderes berechtigtes Interesse etwa dann angenommen werden kann, wenn die namentliche Identität für die Geltendmachung von Ansprüchen oder Rechten aus oder im Zusammenhang mit dem elektronischen Wertpapier im Verhältnis zwischen Inhaber, Berechtigtem, Emittenten und registerführenden Stelle erforderlich ist. ${ }^{47}$ Sollen Ansprüche gerichtlich geltend gemacht werden, hat der Kläger daher ggf. ein Auskunftsrecht gem. \$10 Abs. 3 eWpG.

\section{Problem: Verfügungen über Mischbestand}

\section{aus elektronischen Wertpapieren in Sammeleintragung und Einzeleintragung}

An dieser Stelle sei noch auf ein weiteres Problem hingewiesen: Das eWpG sieht auch die Möglichkeit eines Mischbestands aus elektronischen Wertpapieren in Sammeleintragung und in Einzeleintragung vor ( $\$ 9$ Abs. 3 eWpG). Ein einheitlicher Sammelbestand setzt indes Fungibilität, also gegenseitige Austauschbarkeit der elektronischen Wertpapiere voraus. Erforderlich ist daher, dass auf diese Wertpapiere einheitliche Rechtsregeln zur Anwendung gelangen. ${ }^{48}$ Soweit es um Verfügungsgeschäfte geht, ist diese Voraussetzung bei elektronischen Wertpapieren in Sammeleintragung einerseits und solchen in Einzeleintragung andererseits aber gerade nicht gegeben. So gelten für Verfügungen über elektronische Wertpapiere in Einzeleintragung gem. $\$ \$ \int 24 \mathrm{ff}$. eWpG andere Rechtsvorschriften als für Verfügungen über elektronische Wertpapiere in Sammeleintragung. Auch besteht nur bei elektronischen Wertpapieren in Einzeleintragung die Vermutung der materiellen Berechtigung ( $\$ 27 \mathrm{eWpG}) .{ }^{49}$ Nicht ersichtlich ist daher, wie sich die Vorschrift begründen lässt, dass Wertpapiere in Sammeleintragung und solche in Einzeleintragung einen einheitlichen Sammelbestand bilden können. ${ }^{50}$

47 Vgl. die Begründung RegE zu $₫ 10$ Abs. 3, BT-Drs. 19/26925, S. 52 f.

48 Vgl. hierzu auch Einsele, Wertpapierrecht als Schuldrecht - Funktionsverlust von Effektenurkunden im internationalen Rechtsverkehr (1995), S. 407.

${ }^{49}$ Vgl. hingegen zu sammeleingetragenen elektronischen Wertpapieren unten VII. 1.; zu sammelverwahrten verbrieften Wertpapieren MüKo/Einsele, HGB, Bd.6 (4. Aufl. 2019), Q. Depotgeschäft Rn.148.

50 So aber die Begründung RegE zu $\ 9$ Abs. 3, BT-Drs. 19/26925, S. 51. 


\section{Fiktion des elektronischen Wertpapiers als Sache}

Gemäß $\$ 2$ Abs. 3 eWpG gilt das elektronische Wertpapier als Sache. Damit will der Gesetzgeber die Anwendbarkeit des Sachenrechts auf elektronische Wertpapiere erreichen. ${ }^{51}$ Sind die Kriterien des Sachenrechts, als da wären absolute Herrschaftsmacht des Rechtsinhabers über einen bestimmten Gegenstand sowie dessen absolute und nicht nur relative Offenkundigkeit, ${ }^{52}$ erfüllt, ist die Anwendbarkeit des Sachenrechts auf Nichtsachen in der Tat nicht a priori ausgeschlossen. Allerdings stellen (Gleichstellungs-)Fiktionen im Grunde generell ein Problem dar, und zwar deshalb, weil der wirkliche Sachverhalt ein anderer als der fingierte ist und daher die Regelungen für den fingierten Sachverhalt regelmäßig nicht unmittelbar für den wirklichen Sachverhalt passen; zumindest aber führt die Anwendung dieser Regelungen zu Problemen. Dies gilt auch für elektronische Wertpapiere, die als Sachen fingiert werden. So stellt sich etwa die Frage, wer unmittelbarer bzw. mittelbarer Besitzer (erster, zweiter oder einer höheren Stufe) des elektronischen Wertpapiers sein könnte, inwiefern die Eigentumsvermutung des $\$ 1006$ BGB auch bei elektronischen Schuldverschreibungen (generell) anwendbar ist und ob Besitzschutzansprüche denkbar sind. Ferner stellt sich die Frage, wann der Berechtigte einen Herausgabeanspruch gem. $\$ 985$ BGB hat und unter welchen Voraussetzungen sein Eigentum an einer elektronischen Schuldverschreibung so beeinträchtigt wird, dass dem Berechtigten ein Anspruch aus $\$ 1004$ BGB oder bei Verschulden des Störers ein Anspruch gem. $\ 823$ Abs. 1 BGB zusteht.

\section{Elektronische Wertpapiere in Sammeleintragung}

Im Fall sammeleingetragener elektronischer Wertpapiere ließe sich möglicherweise argumentieren, die Eintragung der Wertpapiersammelbank bzw. des Verwahrers im Wertpapierregister entspreche dem unmittelbaren Besitz an den sammeleingetragenen Wertpapieren. ${ }^{53}$ Immerhin ersetzt die Eintragung auch ansonsten den mit tatsächlicher Sachherrschaft verbundenen Besitz und die formelle Berechtigung an der Urkunde. Lässt sich also in Form der Eintragung als Inhaber ein Pendant zum unmittelbaren Besitz sehen, sind jedoch die Anleger/Berechtigten im Fall der Sammeleintragung nicht etwa quasi mittelbare Besitzer des Sammelbestands. Denn solange die elektronischen Wertpapiere sammeleingetragen sind, hat der Anleger nicht unmittelbar die Möglichkeit, selbst im Wertpapierregister eingetragen und damit quasi Besitzer der elektronischen Wertpapiere zu werden. Zwar sieht $₫ 9 b$ Abs. 2 DepotG vor, dass der Verwah-

\footnotetext{
51 Vgl. Begründung RegE zu $\$ 2$ Abs. 3, BT-Drs. 19/26925, S. 40.

52 Vgl. Einsele, Buchbesprechung von Segna, Ulrich, Bucheffekten - Ein rechtsvergleichender Beitrag zur Reform des deutschen Depotrechts, ZHR 183 (2019), 73, 77.

53 Vgl. so auch Begründung RegE zu $\$ 8$ Abs. 1 Nr. 1, BT-Drs. 19/26925, S. 49.
} 
rer bei Geltendmachung der Auslieferungsansprüche gem. $\int \mathbb{S} 7$ und 8 DepotG den auf den Hinterleger entfallenden Anteil auf dessen Kosten in eine Einzeleintragung zu überführen hat. Dies gilt aber zum einen nur dann, wenn nicht in den Emissionsbedingungen anderes geregelt ist ( $\mathbb{9} 9 \mathrm{~b}$ Abs. 2 DepotG). Zum anderen begründet auch dieser Anspruch auf Einzeleintragung keinen quasi mittelbaren Besitz der Hinterleger an den sammeleingetragenen elektronischen Wertpapieren. Denn im Fall der Überführung einer Sammeleintragung in eine Einzeleintragung ändert sich nicht nur der im Wertpapierregister angegebene Rechtsinhalt (vgl. $\$ 13$ Abs. 1 Nr. 5 eWpG); vielmehr unterliegt ein elektronisches Wertpapier in Einzeleintragung insbesondere auch anderen rechtlichen Regelungen als Wertpapiere in Sammeleintragung (vgl. \$\$ $\$$ 24-27 eWpG). Daher begründet auch dann, wenn der Auslieferungsanspruch gem. $\int \mathbb{S} 7,8$ DepotG in den Emissionsbedingungen nicht ausgeschlossen ist, der Anspruch auf Einzeleintragung keinen mittelbaren Besitz an den sammeleingetragenen Wertpapieren. Damit streitet im Fall der Sammeleintragung auch nicht die Eigentumsvermutung des $\$ 1006$ BGB zugunsten der gerade nicht eingetragenen Anleger. ${ }^{54}$ Mangels (mittelbaren) Besitzes der Anleger sind im Fall der Sammeleintragung Besitzschutzansprüche gegen Dritte, aber auch quasi-Herausgabeansprüche der Berechtigten gem. $\$ 985$ BGB, $\$ 2$ Abs. 3 eWpG nicht denkbar.

Hingegen erscheinen Ansprüche des Berechtigten gem. \$\$ 1004, 823 Abs. 1 BGB durchaus denkbar. Dies ist etwa dann der Fall, wenn in die Übertragung eingeschaltete Intermediäre über das sammeleingetragene elektronische Wertpapier (wirksam) verfügen, ohne dass der materiell berechtigte Anleger die Verfügenden hierzu (im Innenverhältnis) ermächtigt bzw. bevollmächtigt hätte. Fraglich könnte insoweit allerdings sein, ob das sammeleingetragene elektronische Wertpapier überhaupt ein absolutes Recht im Sinn der $\mathbb{\int} \mathbb{S 1 0 0 4 ,} 823$ Abs. 1 BGB darstellt. Denn absolute Rechte müssen von jedermann beachtet werden; sie setzen daher eine Form allgemeiner Erkennbarkeit zumindest in dem Sinn voraus, dass Dritte erkennen können, (eventuell) mit einem fremden Recht zu tun zu haben. Bei sammeleingetragenen elektronischen Wertpapieren wird die Rechtsposition des Berechtigten aber nur aus den dem Bankgeheimnis unterliegenden, nicht einsehbaren Depotbüchern der Wertpapiersammelbank bzw. Verwahrer ersichtlich. Hingegen ist in dem mit Publizitätswirkung ausgestatteten Wertpapierregister nur der Inhaber ( $\mathbb{S} 13$ Abs. 1 Nr.6, 17 Abs. 1 Nr. 6 eWpG), bei Sammeleintragungen also die Wertpapiersammelbank bzw. der Verwahrer, nicht aber der materiell Berechtigte eingetragen. Immerhin ist aber dem Wertpapierregister zu entnehmen, ob es sich um eine Einzel- oder eine Sammeleintragung handelt ( $\mathbb{S} \int 13$ Abs. 1 Nr. 5, 17 Abs. 1 Nr. 5 eWpG). Daher ist für Dritte ersichtlich, dass es sich bei einer Sammeleintragung um ein elektro-

${ }^{54}$ Vgl. auch MüKo/Einsele, HGB, Bd. 6 (4. Aufl. 2019), Q. Depotgeschäft Rn. 148 zu sammelverwahrten verbrieften Wertpapieren. 
nisches Wertpapier handelt, das nicht im Eigentum der Wertpapiersammelbank bzw. des Verwahrers, sondern im Miteigentum der berechtigten Anleger steht. Ansprüche des Berechtigten gem. $\int \mathbb{S} 1004,823$ Abs. 1 BGB wegen einer Beeinträchtigung bzw. Verletzung elektronischer Wertpapiere sind daher möglich.

\section{Elektronische Wertpapiere in Einzeleintragung}

Bei elektronischen Wertpapieren in Einzeleintragung ist der materiell Berechtigte hingegen im Wertpapierregister eingetragen und damit auch quasi unmittelbarer Besitzer des Wertpapiers. Hier besteht zugunsten des Eingetragenen die Eigentumsvermutung gem. \$1006 BGB (so auch $\$ 27 \mathrm{eWpG}$ ). Wird hier dem Eingetragenen ohne seine Zustimmung die Inhaberposition und daher die quasi-Besitzposition entzogen, ohne dass ein Dritter das Wertpapier sodann gutgläubig erwirbt, ist aufgrund der gesetzlichen Fiktion des $\$ 2$ Abs. $3 \mathrm{eWpG}$ ein Anspruch des Berechtigten gem. $\$ 985$ BGB, aber auch etwa der Besitzschutzanspruch auf Wiedereinräumung seiner Inhaberstellung gem. \861 BGB denkbar. Ist mittlerweile ein Dritter in dem Wertpapierregister als Inhaber eingetragen, ohne dass dieser das Wertpapier gutgläubig erworben hätte, kann auch ein Anspruch auf Zustimmung zur Berichtigung des Wertpapierregisters gem. $\$ 1004$ BGB gegeben sein. Sofern indes ein (Zweit-)Erwerber das Eigentum an dem elektronischen Wertpapier mittlerweile gutgläubig erworben hat, ist auch ein Schadensersatzanspruch des Berechtigten gem. $\int \$ 823$ Abs.1, 992 BGB bzw. $\$ 990$ BGB gegen denjenigen möglich, der in seine Position als Inhaber des Wertpapiers rechtswidrig und schuldhaft eingegriffen hat.

\section{Anwendbares Recht}

Die kollisionsrechtliche Regelung des $\ 32 \mathrm{eWpG}$ wurde während des Gesetzgebungsverfahrens geändert und insofern verbessert, als sie nunmehr zwischen einzel- und sammeleingetragenen elektronischen Wertpapieren unterscheidet.

\section{Elektronische Wertpapiere in Einzeleintragung}

Für einzeleingetragene elektronische Wertpapiere sieht $₫ 32 \mathrm{eWpG}$ eine kollisionsrechtliche Sonderregelung vor. Danach unterliegen Rechte an einem elektronischen Wertpapier und Verfügungen über ein elektronisches Wertpapier dem Recht des Staates, unter dessen Aufsicht diejenige registerführende Stelle steht, in deren elektronischem Wertpapierregister das Wertpapier eingetragen ist ( $\ 32$ Abs. $1 \mathrm{eWpG}$. Die Gesetzesbegründung hierzu ist äußerst knapp und nur teilweise erhellend. Immerhin aber findet sich der Hinweis, bei elektronischen Wertpapieren scheide eine Bestimmung des anwendbaren Rechts nach dem Belegenheitsort der Sache (Wertpapierurkunde) aus, auch sei das Wert- 
papierregister bei einer elektronischen Registerführung schwierig zu lokalisieren. ${ }^{55}$

Die Belegenheit der Urkunde ist indes auch bisher nur im Fall der Einzelverwahrung das generelle kollisionsrechtliche Anknüpfungsmoment. So gelangt für sachenrechtliche Fragen bei einzelverwahrten verbrieften Wertpapieren in der Tat die Rechtsordnung zur Anwendung, in der die Urkunde belegen ist (Art. 43 EGBGB). Insofern erscheint die Ersetzung des Anknüpfungsmoments „Belegenheit der Urkunde“ durch den Staat, der den maßgeblichen Registerführer beaufsichtigt, auf den ersten Blick diskutabel.

Im Ergebnis ist die kollisionsrechtliche Anknüpfungsregel des $\$ 32 \mathrm{eWpG}$ aber dennoch problematisch. Noch am wenigsten störend ist insoweit, dass $₫ 32$ eWpG nicht problemlos mit der Gleichstellungsfiktion des $\$ 2$ Abs. 3 eWpG vereinbar ist, wonach ein elektronisches Wertpapier als Sache gilt. Allerdings zeigt die Begründung des RegE zu $₫ 32 \mathrm{eWpG}$ in bemerkenswerter Klarheit, dass das Kriterium der allgemeinen Offenkundigkeit der absoluten Herrschaftsbeziehung des Rechtsinhabers, das ansonsten für Sachen und das Sachenrecht charakteristisch ist, ${ }^{56}$ bei einem elektronischen Wertpapier eben nicht so ohne Weiteres erfüllt ist. Deutlich wesentlicher und zugleich bedenklich ist, dass im Rahmen des $₫ 32$ Abs. 1 eWpG das öffentliche Recht - nämlich der den Registerführer beaufsichtigende Staat - Maß gibt für die internationalprivatrechtliche Frage des auf sachenrechtliche Fragen anzuwendenden Rechts. Eine solche Anknüpfung für die Bestimmung des anwendbaren Privatrechts birgt aber nicht nur generell das Risiko einer inflexiblen Regelung. Gerade im vorliegenden Fall dient sie insbesondere nicht der Verkehrsfähigkeit der elektronisch verkörperten Rechte, sondern ganz im Gegenteil:

Die Bundesanstalt für Finanzdienstleistungsaufsicht (BaFin) überwacht gem. $\$ 11$ eWpG als Aufsichtsbehörde die Führung der elektronischen Wertpapierregister nach dem eWpG. Die Regelungen, wonach in Inhaberschuldverschreibungen repräsentierte Forderungen auch durch Eintragungen in einem elektronischen Wertpapierregister nach dem eWpG „verkörpert“ werden können, dürften indes dann anwendbar sein, wenn für die betreffende Forderung deutsches Recht maßgeblich ist. Denn die Rechtsordnung, die auf das repräsentierte Recht zur Anwendung gelangt, das sog. Wertpapierrechtsstatut, entscheidet über den Inhalt, die Entstehung und den Untergang dieses Rechts, ferner über die Frage, welcher Art ein verkörperndes Wertpapier ist (Inhaber-, Order- oder Rektapapier) und auf welche Weise das verkörperte Recht übertragen werden kann; ${ }^{57}$ das Wertpapierrechtsstatut entscheidet damit auch über

55 Vgl. Begründung RegE zu $\$ 32$ eWpG, BT-Drs. 19/26925, S. 69.

56 Vgl. auch zu den denkbaren Möglichkeiten der Bestimmung des Sachstatuts für digitale Vermögenswerte, Wendehorst, Digitalgüter im internationalen Privatrecht, IPRax 2020, 490, 494-498.

57 Vgl. statt vieler BGHZ 108, 353, 356; MüKo/Einsele, HGB, Bd.6 (4. Aufl. 2019), 
die Frage, ob das verkörperte Recht durch Übertragung des (elektronischen) Wertpapiers (sog. Wertpapiersachstatut) übertragen werden kann. ${ }^{58}$ Insoweit regelt $\$ 32 \mathrm{eWpG}$ das elektronische Wertpapiersachstatut. Da für die elektronischen Wertpapierregister nach dem eWpG aber die BaFin Aufsichtsbehörde ist, unterliegen Rechte an einem elektronischen Wertpapier und Verfügungen über ein elektronisches Wertpapier nach $\$ 32$ Abs. $1 \mathrm{eWpG}$ stets deutschem Recht.

Allerdings macht insbesondere $\$ 32$ Abs. 2 eWpG deutlich, dass die kollisionsrechtliche Regelung des $\$ 32 \mathrm{eWpG}$ nicht nur elektronische Schuldverschreibungen nach dem eWpG, sondern auch solche Schuldverschreibungen erfasst, bei denen das elektronisch „verkörperte“ Recht ausländischem Recht untersteht. Sofern hier die registerführende Stelle nicht unter Aufsicht steht, ist gem. $\$ 32$ Abs. 2 eWpG der Sitz der registerführenden Stelle bzw. bei Unbestimmbarkeit der registerführenden Stelle der Sitz des Emittenten des elektronischen Wertpapiers maßgebend. Vorrangig ist aber auch für Wertpapiere mit ausländischem Wertpapierrechtsstatut das Recht des Staates anwendbar, unter dessen Aufsicht die registerführende Stelle steht, in deren elektronischem Wertpapierregister das Wertpapier eingetragen ist ( $\$ 32 \mathrm{Abs.} 1 \mathrm{eWpG})$. Da die BaFin für ausländische elektronische Wertpapiere wohl kaum Aufsichtsbehörde sein wird, findet gem. $\$ 32 \mathrm{Abs.} 1 \mathrm{eWpG}$ nicht das deutsche Recht, sondern das Recht des ausländischen Aufsichtsstaats Anwendung. Im Ergebnis gilt daher: Für elektronische Wertpapiere nach dem eWpG ist das Wertpapiersachstatut stets das deutsche Recht. Bei Wertpapieren mit ausländischem Wertpapierrechtsstatut lassen sich zwar keine allgemeinen Aussagen treffen. Jedenfalls bei ähnlichen Kompetenzzuweisungen wie nach dem eWpG wird aber auch bei diesen Wertpapieren das maßgebliche Wertpapiersachstatut nach $₫ 32 \mathrm{eWp}$ regelmäßig zugleich deren Wertpapierrechtsstatut sein.

Mit der Verbriefung von Rechten sollte eigentlich deren Verkehrsfähigkeit gesteigert werden. Die Regelanknüpfung des $\$ 32 \mathrm{Abs.} 1 \mathrm{eWpG}$ bewirkt jedoch das Gegenteil, weil Verfügungen über das elektronische Wertpapier im Allgemeinen dem Recht des Heimatstaats des Wertpapiers unterliegen werden. Dass privatrechtliche Rechtspositionen sich kollisionsrechtlich (vorrangig) nach öffentlich-rechtlichen Kompetenzzuweisungen richten, dürfte also kaum ein Schritt in Richtung Verkehrsfähigkeit elektronischer Rechte im Besonderen und Internationalisierung des Rechts im Allgemeinen sein.

Q. Depotgeschäft Rn.15; MüKo/Wendehorst, BGB, Bd.12 (8. Aufl. 2020), Art. 43 EGBGB Rn. $200 \mathrm{f}$.

${ }^{58}$ Vgl. zu dem Zusammenspiel von Wertpapierrechtsstatut und Wertpapiersachstatut auch Einsele, Das Kollisionsrecht Intermediär-verwahrter Wertpapiere, EuZW 2018, 402, 403. 


\section{Elektronische Wertpapiere in Sammeleintragung}

Sofern es hingegen um sammeleingetragene elektronische Wertpapiere geht, erklärt $\$ 32$ Abs. 1 eWpG nunmehr vorrangig $\$ 17$ a DepotG für anwendbar. ${ }^{59}$ So unklar der Bedeutungsgehalt von $\$ 17$ a DepotG auch ist, hat das eWpG damit - anders als noch der RegE ${ }^{60}$ - wenigstens einen Verstoß gegen gleich mehrere europäische Richtlinien vermieden. Konkret geht es dabei zum einen um Art. 9 Abs. 2 der Richtlinie 98/26/EG des Europäischen Parlaments und des Rates v. 19.5.1998 über die Wirksamkeit von Abrechnungen in Zahlungs- sowie Wertpapierliefer- und -abrechnungssystemen ${ }^{61}$ (sog. Finalitätsrichtlinie). Vom Wortlaut dieser Bestimmung werden zwar lediglich Sicherungsgeschäfte über Wertpapiere erfasst. Allerdings ist der Begriff der dinglichen Sicherheit gem. Art. $2 \mathrm{~m}$ ) weit zu verstehen und erfasst insbesondere auch Sicherungsübereignungen sowie Pensionsvereinbarungen. ${ }^{62} \mathrm{Zum}$ anderen handelt es sich um Art. 9 der Richtlinie 2002/47/EG des Europäischen Parlaments und des Rates über Finanzsicherheiten v. 6.6.2002 ${ }^{63}$ (Finanzsicherheitenrichtlinie). Diese Richtlinie bezweckt ebenfalls, die Rechtssicherheit im Bereich der Finanzsicherheiten zu erhöhen und möchte hierzu die kollisionsrechtliche Vorschrift der Finalitätsrichtlinie ausdehnen. ${ }^{64}$ Zwar ist der persönliche und sachliche Anwendungsbereich auch dieser Richtlinie eingeschränkt. ${ }^{65}$ Dennoch sind im Bereich des

\footnotetext{
${ }^{59}$ Vgl. hierzu Beschlussempfehlung und Bericht des Finanzausschusses zum RegE BTDrs. 19/29372, S. 61.

60 Vgl. RegE zu $\$ 32$ eWpG BT-Drs. 19/26925, S. 21.

61 Richtlinie (EG) Nr. 98/26/EG vom 19.05.1998, ABl. EG Nr. L 166, S. 45, mittlerweile geändert durch die Richtlinie 2009/44/EG des Europäischen Parlaments und des Rates vom 06.05.2009, ABl. EU Nr. L 146, S. 37.

${ }^{62} \mathrm{Vgl}$. hierzu auch Schefold, Grenzüberschreitende Wertpapierübertragungen und internationales Privatrecht - zum kollisionsrechtlichen Anwendungsbereich von $\$ 17$ a DepotG, IPRax 2000, 468, 473; Keller, Die EG-Richtlinie 98/26 vom 19. 5. 1998 über die Wirksamkeit von Abrechnungen in Zahlungs- sowie Wertpapierliefer- und -abrechnungssystemen und ihre Umsetzung in Deutschland, WM 2000, 1269, 1273; so auch die österreichische Literatur, vgl. Jergitsch, Finalität als Schutz vor systemischen Risken in Zahlungs- und Wertpapierliefersystemen, ÖBA 1999, 537, 543.

${ }^{63}$ Richtlinie (EG) Nr. 2002/47/EG vom 06.06.2002, ABl. EG Nr. L. 168, S. 43, mittlerweile geändert durch die Richtlinie 2009/44/EG des Europäischen Parlaments und des Rates vom 06.05.2009, ABl. EU Nr. L 146, S. 37.

${ }^{64}$ Vgl. hierzu 5. und 7. Erwägungsgrund der Finanzsicherheitenrichtlinie.

65 Von der Finanzsicherheitenrichtlinie werden ebenfalls nur Sicherungsrechte erfasst, und zwar solche an Finanzinstrumenten. Der Begriff der Finanzinstrumente ist allerdings weit zu verstehen. Hierzu zählen gem. Art. 2 Abs. 1e) Aktien und andere, diesen gleichzustellende Wertpapiere, Schuldverschreibungen und sonstige verbriefte und unverbriefte Schuldtitel, die auf dem Kapitalmarkt gehandelt werden können (etc.), daneben aber auch jegliche Rechte oder Ansprüche im Zusammenhang mit irgendeinem der vorgenannten Aktiva. Der Begriff der Sicherheiten ist ebenfalls recht weit gefasst und schließt wiederum Vollrechtsübertragungen zu Sicherungszwecken ein (vgl. Art.2 Abs.1a) u. b)). Der persönliche Anwendungsbereich der Richtlinie ist allerdings erheblich eingeschränkt. Denn die Finanzsicherheitenrichtlinie regelt nur Sicherungsrechte zwischen bestimmten Sicherungsnehmern
} 
Effektengiroverkehrs die Vorgaben des Art. 9 Abs. 1 der Finanzsicherheitenrichtlinie zu beachten. Und danach sollen Verfügungen über „im Effektengiroverkehr übertragbare Wertpapiere“ dem Recht des Landes unterstehen, in dem das maßgebliche Konto i.S.d. Art. 2 Abs. 1h) der Richtlinie geführt wird. Dabei sind „im Effektengiro übertragbare Wertpapiere“ gem. Art. 2 Abs.1g) der Richtlinie Finanzsicherheiten in Form von Finanzinstrumenten, bei denen die Eigentumsverhältnisse durch einen Registereintrag oder eine Buchung auf einem von einem Intermediär oder für den Intermediär selbst geführten Depotkonto nachgewiesen wird. Von der kollisionsrechtlichen Regelung des Art. 9 der Finanzsicherheitenrichtlinie ausgenommen sind daher Wertpapiere, die direkt vom Anleger gehalten werden, ${ }^{66}$ mithin also auch elektronische Wertpapiere in Einzeleintragung. ${ }^{67}$ Das anwendbare Recht bestimmt sich im Übrigen nach dem maßgeblichen Konto. Hierbei handelt es sich um das Register oder Depotkonto - das auch vom Sicherungsnehmer selbst geführt werden kann -, in dem der maßgebliche Eintrag bzw. auf dem die maßgebliche Buchung erfolgt, auf Grund deren der Sicherungsnehmer die Sicherheit erlangt. ${ }^{68}$

So unklar diese europäischen Richtlinien und insbesondere auch die deutsche Umsetzungsregelung des $₫ 17$ a DepotG im Einzelnen auch sind, ${ }^{69}$ eines ist klar: Der gem. $\ 32$ Abs. $1 \mathrm{eWpG}$ kollisionsrechtlich vorrangig relevante Führer des Wertpapierregisters ist bei grenzüberschreitenden Intermediärs- und Verwahrketten nicht zwangsläufig die Stelle, die i.S.v. \$17a DepotG zugunsten des Verfügungsempfänger die maßgebliche, rechtsbegründende Depotbuchung vornimmt. Daher sieht $\$ 32$ Abs. $1 \mathrm{eWpG}$ - anders als noch der Regierungsent-

und Sicherungsgebern, nämlich insbes. öffentlichen Körperschaften (Art. 1 Abs. 2a)), Zentralbanken (Art. 1 Abs. 2b)) sowie beaufsichtigten Finanzinstituten, namentlich Kreditinstituten (vgl. Art. 1 Abs. 2c)). Zwar werden von der Finanzsicherheitenrichtlinie im Grundsatz auch sonstige juristische Personen des Privatrechts, Einzelkaufleute und Personengesellschaften erfasst (Art. 1 Abs. 2e)). Allerdings gelten die Regelungen der Richtlinie für diese Personengruppe nur dann, wenn die andere Vertragspartei einer der sonstigen, unter die Richtlinie fallenden Personengruppen angehört (also etwa Kreditinstitut und nicht selbst ebenfalls eine Personengesellschaft oder ein Einzelkaufmann ist).

66 So auch Segna, Bucheffekten - Ein rechtsvergleichender Beitrag zur Reform des deutschen Depotrechts (2018), S. 375.

67 Vgl. auch Ebenroth/Boujong/Joost/Strohn/Scherer, HGB, Bd 2 (4. Aufl. 2020), $\$ 17$ a DepotG Rn. 3 für den Fall sonderverwahrter Wertpapiere; vgl. auch die Begründung BRDrs. 456/99, S.35, wonach $\$ 17$ a DepotG auf sonderverwahrte Wertpapiere keine Anwendung findet, da im Fall der Sonderverwahrung die Depotgutschrift nicht rechtsbegründend wirkt, sondern den Eigentumsübergang, der nach allgemeinen Vorschriften stattfindet, nur dokumentiert.

68 In diesem Fall entspricht auch die englische Fassung der deutschen: „, relevant account means in relation to book entry securities collateral which is subject to a financial collateral arrangement, the register or account - which may be maintained by the collateral taker - in which the entries are made by which that book entry securities collateral is provided to the collateral taker".

69 Vgl. dazu auch Einsele, EuZW 2018, 402, $404 \mathrm{ff}$. 
wurf - zu Recht die vorrangige Anwendbarkeit von $\$ 17$ a DepotG auf sammeleingetragene elektronische Wertpapiere vor.

\section{Fazit}

Ziel des eWpG ist, elektronische Wertpapiere entsprechend den $\$ \$ 793 \mathrm{ff}$. BGB zu regeln. Dies gelingt indes nur teilweise. Der wesentliche Grund hierfür ist aber nicht die Tatsache, dass die Repräsentation der Rechte im Fall elektronischer Wertpapiere durch Registereintragungen erfolgt. Überhaupt liegt das Hauptproblem des modernen Wertpapierverwahrungs-, -übertragungs-, und -verwaltungsrechts nicht in dem Medium der Repräsentation der verkörperten Rechte. Die Diskussion um das Verkörperungsmedium war und ist vielmehr ein „Nebenkriegsschauplatz“. Die wesentliche Trennlinie verläuft nicht zwischen elektronischen und verbrieften Wertpapieren, sondern zwischen Wertpapieren im Sammelbestand - seien sie verbrieft oder elektronisch - und Wertpapieren in Sonderverwahrung bzw. Einzeleintragung.

Das eWpG trägt diesem Umstand zwar deutlich mehr Rechnung, als dies noch bei dem Regierungsentwurf der Fall war. Dies zeigen insbesondere die nunmehr Gesetz gewordenen Regelungen der $\$ \$ 28,32 \mathrm{eWpG}$. Diese Vorschriften rufen zwar im Einzelnen immer noch Kritik hervor, unterscheiden aber richtigerweise zwischen elektronischen Wertpapieren in Einzeleintragung und solchen in Sammeleintragung. Die Probleme, die sammelverwahrte bzw. sammeleingetragene Wertpapiere sowie die Intermediatisierung der Wertpapierverwahr- und übertragungssysteme aufwerfen, werden hierdurch allerdings nicht gelöst. Symptomatisch für die dadurch verursachten Probleme ist nicht zuletzt die mit dem Gesetz zur Einführung von elektronischen Wertpapieren neu aufgenommene, eher verunglückte Regelung der Depotbescheinigung zur Rechtsausübung ( $\$ 6$ Abs. 2 DepotG). Eine sinnvolle Reform des Depotrechts müsste indes die rechtlichen Konsequenzen regeln, die sich aus der Entwicklung zur Sammelverwahrung und nunmehr auch Sammeleintragung von Wertpapieren sowie der Intermediatisierung ergeben. Dies geschieht mit dem eWpG jedoch nicht. 


\title{
Wertpapierarten
}

\author{
Matthias Lehmann
}

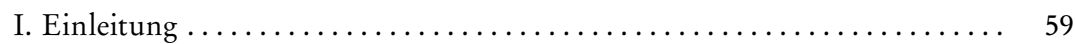

II. Elektronische Wertpapiere im Anwendungsbereich des eWpG ........ 60

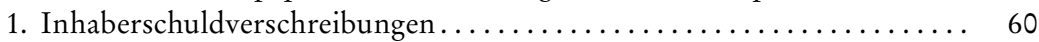

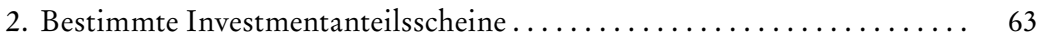

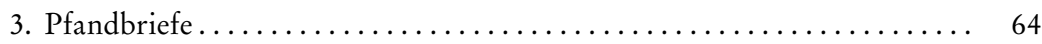

III. Die Wertpapiere und Finanzinstrumente außerhalb des

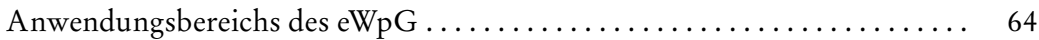

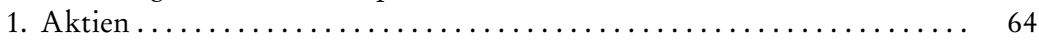

2. Anteile an Investmentaktien- und Investmentkommanditgesellschaften . 67

3. Zwischenscheine, Bezugsscheine und andere mit Aktien vergleichbare

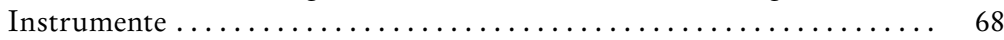

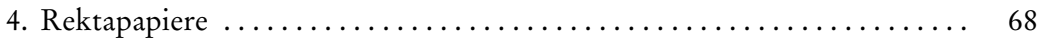

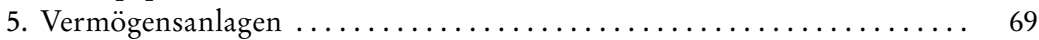

6. Derivate ......................................... 69

IV. Entmaterialisierte Werte und Wertpapiere außerhalb des eWpG....... 70

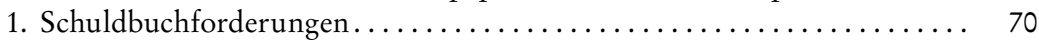

2. Handelsrechtliche Wertpapiere..................... 71

V. Perspektiven einer einheitlichen Regelung elektronischer Wertpapiere .... 72

1. Integration der verschiedenen Texte in einem Gesetzbuch . . . . . . 72

2. Einbeziehung aller Arten von Wertpapieren .............. 74

3. Erweiterung auf andere Gegenstände als Wertpapiere .......... 75

VI. Die neue Nomenklatur der Wertpapiere ................... 76

1. Physische und elektronische Wertpapiere ................. 76

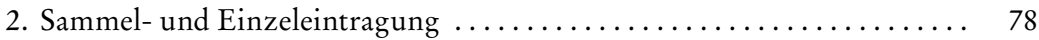

3. Übertragung der herkömmlichen Nomenklatur auf Zentralregister-

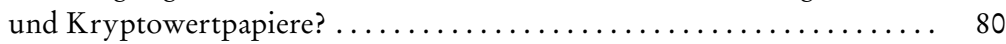

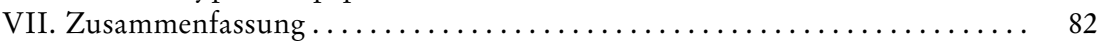

\section{Einleitung}

Das eWpG wird das Wertpapierrecht revolutionieren, allerdings nur in kleinen Schritten. Die Einführung elektronischer Wertpapiere durch das Gesetz erfolgt lediglich innerhalb seines Anwendungsbereichs, der viel bescheidener ist, als 
sein weit gefasster Titel vermuten lässt. Entgegen dem dort verwendeten Begriff regelt dieses nicht alle „Wertpapiere“, sondern nur solche, die der Unternehmensfinanzierung dienen und am Kapitalmarkt gehandelt werden; und selbst von diesen Instrumenten erfasst es nur einen kleinen Teil.

Um die praktische Bedeutung des Gesetzes zutreffend einschätzen zu können, bedarf es daher einer genauen Analyse der von ihm erfassten Wertpapierarten (II.). Anhand dieser lässt sich auch überprüfen, welche Instrumente nach wie vor in Papierform begeben werden müssen, und ob die Gründe für deren Ausschluss vom Anwendungsbereich des neuen Gesetzes stichhaltig sind (III.). Außerdem weckt der Blick auf die vom eWpG erfassten Wertpapierarten die Aufmerksamkeit dafür, dass auch außerhalb dessen Anwendungsbereichs verschiedene Formen elektronischer Wertpapiere existieren (IV.). Dies wirft die Frage auf, welche Perspektiven für eine einheitliche Regelung aller elektronischen Wertpapiere bestehen (V.). Schließlich ist zu analysieren, welche Gestalt das neue System des Wertpapierrechts hat, insbesondere wie die Nomenklatur der Wertpapierarten ausgestaltet ist (VI.).

\section{Elektronische Wertpapiere im Anwendungsbereich des eWpG}

In den ausdrücklichen Anwendungsbereich des eWpG fallen nur Inhaberschuldverschreibungen (1.). Darüber hinaus ist es auch für bestimmte Investmentanteilsscheine (2.) und Pfandbriefe (3.) bedeutsam.

\section{Inhaberschuldverschreibungen}

Gegenstand des eWpG sind gemäß dessen $\$ 1$ „Schuldverschreibungen auf den Inhaber“. Damit wird auf die in $\$ 793$ BGB geregelte Wertpapierart verwiesen. Andere Wertpapiere, selbst insoweit sie am Kapitalmarkt gehandelt werden, sind nicht erfasst.

Warum wurde gerade die Inhaberschuldverschreibung für die Einführung elektronischer Wertpapiere ausgewählt? Die Begründung des Regierungsentwurfs verweist darauf, dass „ein großes praktisches Bedürfnis nach dieser Finanzierungsform “ bestehe. ${ }^{1}$ In der Tat werden Schuldverschreibungen bereits seit 2019 auf dem deutschen Markt mittels Blockchain begeben ${ }^{2}$ und auch von etablierten Unternehmen zur Finanzierung eingesetzt. ${ }^{3} \mathrm{Zu}$ berücksichtigen

${ }^{1}$ Bundesregierung, Begründung zum Entwurf eines Gesetzes zur Einführung von elektronischen Wertpapieren, 2020, S. 41 (zu \$1).

2 Siehe https://www.bitbondsto.com/de/ (alle Hyperlinks in diesem Beitrag wurden zuletzt am 20.05.2021 abgerufen).

3 Beispielsweise begab die FR L'Osteria SE eine tokenbasierte, qualifiziert nachrangige Schuldverschreibung, https://www.kapilendo.de/projekte/b09f4821-4618-422b-b065Od41d9452656? partner=kap-b\&kbid=9826\&aid=bc04\#/. 
ist insoweit jedoch, dass das eWpG nur „verbriefte“ Obligationen erfasst, d.h. solche, über die ein Wertpapier ausgestellt wird. Nicht abgedeckt sind die am Kapitalmarkt besonders häufigen Schuldscheindarlehen, die zu einer beliebten Finanzierungsform des deutschen Mittelstands geworden sind. ${ }^{4}$ Bei ihnen handelt es sich um gewöhnliche Darlehen i. S. d. SS $488 \mathrm{ff}$. BGB, über die ein Schuldschein i.S.d. $\$ 371$ BGB ausgestellt wird. Dieser ist allerdings kein Wertpapier; das Eigentum am Schuldschein folgt vielmehr im Einklang mit $\$ 952$ Abs. 1 BGB der Inhaberschaft der Forderung.

Die Konzentration des eWpG auf die Schuldverschreibung ist aus ökonomischer Sicht fragwürdig. Das neue Gesetz erleichtert die elektronische Begebung von Fremdkapital; im Interesse einer stabilen Wirtschaft liegt hingegen die Förderung der Begebung von Eigenkapital. ${ }^{5}$ Durch die einseitige Fokussierung auf Fremdkapitalinstrumente riskiert der Gesetzgeber eine weitere gefährliche Schlagseite der Unternehmensfinanzierung in Richtung Verschuldung statt einer Stärkung der Eigenkapitalbasis.

Tatsächlich dürfte - entgegen dem Regierungsentwurf - der Grund für die Wahl der Inhaberschuldverschreibung nicht ein Bedürfnis der Praxis, sondern ein anderer sein. Aufschlussreich ist insoweit die Erklärung der Bundesregierung für den Ausschluss der Eigenkapitalfinanzierung: Sie begründet diesen mit den erheblichen Auswirkungen, die mit der Regulierung von elektronischen Aktien verbunden seien. ${ }^{6}$ Die Fokussierung auf Inhaberschuldverschreibungen ist demzufolge vor allem der Ansicht des Gesetzgebers geschuldet, dass es sich bei ihnen um verhältnismäßig einfach strukturierte Wertpapiere handelt, deren Regelung keine weitergehenden Auswirkungen auf das Zivilrecht hat.

In der Tat ist die Inhaberschuldverschreibung geradezu ein Grundtypus des Wertpapiers. Die Regelung in $\$ \int 793 \mathrm{ff}$. BGB enthält alle Wesensmerkmale des Wertpapiers und wird oft als Vorbild für die lehrbuchartige Darstellung des Wertpapierrechts genutzt. ${ }^{7}$ Dies darf jedoch nicht zur Unterschätzung der Komplexität der Inhaberschuldverschreibung verleiten.

Zum einen sind die Regelungen der $\$ \int 793 \mathrm{ff}$. BGB keineswegs die einzigen, die auf diese Wertpapierart Anwendung finden. Zusätzlich sind etwa die Regelungen der $\iint 305 \mathrm{ff}$. BGB über Allgemeine Geschäftsbedingungen zu beachten. Außerdem gelten das Schuldverschreibungsgesetz und für die Hinterlegung in der Sammelverwahrung das Depotgesetz.

${ }^{4} \mathrm{Zu}$ Schuldscheindarlehen siehe MüKo/Singhof, HGB, Bd.6 (4. Aufl. 2019), L. Emissionsgeschäft Rn. 140; Webrhabn, Unternehmensfinanzierung durch Schuldscheindarlehen, BKR 2021, 363; Woesch/Dietrich, Mustervertrag für Schuldscheindarlehen nach LMA-Standard, BKR 2019, 399.

${ }^{5}$ Siehe Admati/Hellwig, The Banker's New Clothes (2013), S. $94 \mathrm{ff}$.

6 Siehe Bundesregierung, Begründung zum Entwurf eines Gesetzes zur Einführung von elektronischen Wertpapieren (2020), S. 41 (zu $\$ 1)$.

7 Siehe z. B. BeckOGK/Vogel, BGB (01.01.2021), \$793 BGB Rn. 2-73; MüKo/Habersack, BGB, Bd.7 (8. Aufl. 2020), vor $\$ 793$. 
Zum anderen deckt der Begriff der „Inhaberschuldverschreibung“ neben den klassischen Anleihen eine Reihe weiterer Produkte ab. Dazu gehören z. B. Genussscheine: Obwohl das Aufsichtsrecht diese als Vermögensanlagen einordnet, ${ }^{8}$ sind sie zivilrechtlich gesehen Inhaberschuldverschreibungen ${ }^{9}$ und fallen daher in der Regel unter das eWpG. ${ }^{10}$ Genauso verhält es sich mit verbrieften Optionen (Optionsscheinen), die ebenfalls als Inhaberschuldverschreibungen qualifiziert werden. ${ }^{11}$ Am Geldmarkt gehandelte commercial papers werden gleichfalls unter $\ 793$ BGB gefasst, ${ }^{12}$ ebenso wie die zahllosen in der Praxis angebotenen „Zertifikate“. ${ }^{13}$ Inhaberschuldverschreibungen sind zudem creditlinked notes, exchange traded notes und exchange traded commodities, ${ }^{14}$ sowie darüber hinaus strukturierte Produkte wie asset-backed securities (ABS) (soweit nicht als Aktien begeben $)^{15}$ und collateralized debt obligations (CDOs). Auch die in $\$ 221 \mathrm{AktG}$ genannten Wandel- und Gewinnschuldverschreibungen sind im Kern Inhaberschuldverschreibungen ${ }^{16}$ und fallen daher in den Anwendungsbereich des eWpG. Bei Wandelschuldverschreibungen ist dies besonders delikat, da sie in Aktien umgewandelt werden können, die ihrerseits nicht vom eWpG erfasst sind. Neben dem elektronischen Wertpapier der Wandelschuldverschreibung muss daher stets auch noch eine papierne Aktien(sammel)urkunde bestehen, um eine Wandlung ermöglichen zu können.

Insgesamt ist der Anwendungsbereich des eWpG also beträchtlich weiter, als die Bezeichnung „Inhaberschuldverschreibung“ suggeriert. Hinter dem Terminus verbirgt sich ein ganzes Universum verschiedener Instrumente. Diese weichen vom Standardmodell des $\$ 793$ BGB zum Teil sehr weit ab und können rechtlich äußerst komplex sein. Die vermeintliche „Einfachheit“ der Inhaberschuldverschreibung ist daher kein zutreffendes Argument für eine Begrenzung der Einführung elektronischer Wertpapiere auf diesen Archetyp des Wertpapiers.

${ }^{8} \$ 1$ Abs. 2 Nr.7 VermAnlG.

9 MüKo/Habersack, BGB, Bd. 7 (8. Aufl. 2020), \$793 BGB Rn. 27.

10 So ausdrücklich Bundesregierung, Begründung zum Entwurf eines Gesetzes zur Einführung von elektronischen Wertpapieren (2020), S. 41 (zu $\$ 1$ ).

11 BGH, ZIP 1994, 1924 (1925); BeckOGK/Vogel, BGB (01.01.2021), \793 BGB Rn. 123; Staudinger/Marburger (2015), Vorbem. zu \$S793-808 Rn.69; Dierks, Selbständige Aktienoptionsscheine (2000), S. 29.

12 Assmann/Schneider/Mülbert/Assmann, Wertpapierhandelsrecht (7. Aufl. 2019), $\$ 2$ WpHG Rn. 43; Schwark/Zimmer/Kumpan, Kapitalmarktrechts-Kommentar (5. Aufl. 2020), \$2 WpHG, Rn. 34.

13 Lehmann, Finanzinstrumente (2010), S. 110.

${ }^{14}$ Schwark/Zimmer/Kumpan, Kapitalmarktrechts-Kommentar (5. Aufl. 2020), $\$ 2$ Rn. 58 WpHG; Schwark/Zimmer/Röh, Kapitalmarktrechts-Kommentar (5. Aufl. 2020), \$79 WpHG Rn.24; für CLN: Jahn/Reiner, in: Schimansky/Bunte/Lwowski (Hrsg.), Bankrechts-Handbuch (5. Aufl. 2017), \$114 Rn.33; MüKo/Zabrte, HGB, Bd. 6 (4. Aufl. 2019), M. Anlageberatung Rn. 346; Brandt, Kreditderivate - Zentrale Aspekte innovativer Kapitalmarktprodukte, BKR 2002, 243, 244.

15 Lebmann, Finanzinstrumente (2010), S. 128.

16 MüKo/Habersack, BGB, Bd. 7 (8. Aufl. 2020), \$793 BGB Rn. 15f., 25. 


\section{Bestimmte Investmentanteilsscheine}

Die zweite Art von Wertpapieren, für die die elektronische Begebung eingeführt wird, sind Investmentanteilsscheine. Dies geschieht durch eine Änderung des Kapitalanlagegesetzbuchs (KAGB). In \95 KAGB werden nunmehr die elektronischen Anteilsscheine erwähnt und den verbrieften gleichgestellt. Allerdings wird zugleich angeordnet, dass elektronische Anteilsscheine stets auf den Inhaber lauten müssen. ${ }^{17}$ Namensanteilsscheine können daher nicht elektronisch begeben werden.

Technisch gesehen werden elektronische Anteilsscheine nicht im eWpG geregelt, sondern dessen Bestimmungen sind auf elektronische Anteilsscheine entsprechend anwendbar. ${ }^{18}$ Der Verweis gilt zudem nicht für alle Vorschriften des eWpG. Ausgenommen sind unter anderem die Bestimmungen über die Wertpapiersammelbank, die Emissionsbedingungen und das zentrale Register, ${ }^{19}$ die für Anteile an Investmentsondervermögen nicht passen. Ebenfalls ausgenommen sind alle Bestimmungen des eWpG über Kryptowertpapiere. ${ }^{20}$ Investmentanteilsscheine können daher nach deutschem Recht nicht auf der Blockchain begeben werden, sondern - soweit eine digitale Form gewählt werden soll - lediglich als elektronisches Zentralregisterwertpapier.

Zudem können Anteilsscheine nur über Anteile an Sondervermögen ausgestellt werden. ${ }^{21}$ Erfasst sind folglich nur inländische offene Investmentvermögen in Vertragsform. ${ }^{22}$ Nicht der elektronischen Form fähig sind dagegen Beteiligungen an Investmentgesellschaften, d. h. Investmentaktiengesellschaften und Investmentkommanditgesellschaften, gleich ob mit fixem oder veränderlichem Kapital, denn diese bilden keine Sondervermögen. Dies steht im Einklang mit dem generellen Ausschluss von Unternehmensbeteiligungen (Eigenkapital) aus dem Anwendungsbereich des eWpG. ${ }^{23}$ Ebenfalls nicht erfasst sind geschlossene Investmentfonds, wie z. B. Immobilienfonds. Dies kann damit erklärt werden, dass diese nicht dem öffentlichen Handel am Kapitalmarkt unterliegen, sondern nur im Wege der Privatplatzierung vertrieben und von Anleger zu Anleger gehandelt werden. Allerdings wäre gerade hier die rechtssichere Übertragung (peer to peer) besonders wichtig.

Insgesamt bleibt damit ein Großteil der Investmentfondstypen außerhalb des Anwendungsbereichs des eWpG. Dies ist zu bedauern, denn auch für die

17 \$95 Abs. 1 S. 2 KAGB.

18 \$95 Abs. 3 KAGB.

19 Siehe die Nichterwähnung von $₫ 4$ Abs. 5 und 7, $\$ 5$ sowie des Abschnitts $2 \mathrm{eWpG}$ in \95 Abs. 3 KAGB.

20 Siehe die Nichterwähnung von $\ 4$ Abs. 1 Nr.2, Abs. 3 sowie des Abschnitts 3 eWpG in $\$ 95$ Abs. 3 KAGB.

21 \$95 Abs. 1 S. 1 KAGB.

22 Siehe $\$ 1$ Abs. 10 KAGB.

${ }^{23}$ Dazu oben II.1. 
Beteiligungen an Investmentgesellschaften und geschlossenen Fonds bietet insbesondere die Übertragung mittels Kryptotechnologie Vorteile.

\section{Pfandbriefe}

Pfandbriefbanken geben gedeckte Schuldverschreibungen aus. ${ }^{24}$ Deren einzige Besonderheit gegenüber „normalen“ Inhaberschuldverschreibungen besteht darin, dass sie durch einen sog. Deckungsstock bestehend aus Hypothekenpfandbriefen, öffentlichen Pfandbriefen, Schiffspfandbriefen und/oder Flugzeugpfandbriefen gedeckt sind. ${ }^{25}$ Daher geht der Gesetzgeber davon aus, dass die von Pfandbriefbanken begebenen gedeckten Schuldverschreibungen in den Anwendungsbereich des eWpG fallen, sofern sie auf den Inhaber und nicht auf den Namen lauten. Die gedeckte Inhaberschuldverschreibung unterliegt qua ihrer Natur als Schuldverschreibung sämtlichen Vorschriften des eWpG. Eines Verweises bedarf es anders als bei den Investmentanteilsscheinen nicht.

Ausnahmen von der Anwendbarkeit der Regelungen des eWpG sind nicht vorgesehen. Der Gesetzgeber passt das PfandBG lediglich an die neuen elektronischen Pfandbriefe an. Die dazu notwendigen Änderungen sind allerdings marginal. Der neue $₫ 4$ Abs. 5 S. 2 PfandBG regelt den Zeitpunkt, zu dem der Pfandbrief in den Umlauf gelangt. Die in $\$ 4$ Abs. 5 PfandBG vorgesehene Ausfertigung und Übergabe an die Pfandbriefbank passt auf elektronische Wertpapiere nicht. Sie wird daher durch die Ausstellung der Bescheinigung über die Deckung durch den Treuhänder ${ }^{26}$ ersetzt. Diese Bescheinigung ist nach dem neuen $\int 8$ Abs. 3 S. 3 PfandBG bei der Stelle niederzulegen, die das elektronische Wertpapierregister führt und bei der auch die Emissionsbedingungen des Pfandbriefs niedergelegt sind.

Zusammengefasst sind gedeckte Inhaberschuldverschreibungen „normale“ Inhaberschuldverschreibungen i.S.d. $\$ 1 \mathrm{eWpG}$, das auf diese damit voll anwendbar ist. Insoweit bestehen keine Besonderheiten.

\section{Die Wertpapiere und Finanzinstrumente außerhalb des Anwendungsbereichs des eWpG}

\section{Aktien}

Nicht vom eWpG erfasst werden Eigenkapitalinstrumente, selbst wenn sie am Kapitalmarkt gehandelt werden. Das gilt insbesondere für die Aktie. Deren Ausschluss ist besonders misslich, denn die Aktie ist als Instrument für die Stärkung des Eigenkapitals von Unternehmen besonders bedeutsam. ${ }^{27}$ Eine

${ }^{24}$ Vgl. $\ 1$ Abs. 1 S. 2 Nr. 1 Pfandbriefgesetz (PfandBG).

25 Vgl. $\$ 1$ Abs. 1 PfandBG.

26 Siehe zu dieser $\$ 8$ Abs. 3 PfandBG.

27 Siehe bereits oben II.1. 
elektronische Form der Begebung könnte die Attraktivität deutscher Aktien für ausländische Investoren steigern. Gerade bei grenzüberschreitenden Aktientransaktionen existieren unter dem bestehenden Effektengirosystem zahlreiche Schwierigkeiten, die zu Rechtsrisiken und Informationskosten führen. ${ }^{28}$ Das deutsche Modell, welches auf der Notwendigkeit einer physischen Urkunde aufbaut und für die Übertragung den Besitzübergang verlangt, ist dabei aus internationaler Sicht eigentümlich. ${ }^{29}$ Eine Modernisierung im Sinne einer Entmaterialisierung würde das deutsche Recht auf den Stand entwickelter Industrieländer bringen und zahlreiche Friktionen im Verhältnis zu anderen Rechtsordnungen vermeiden. Dies käme auch der Attraktivität des Finanzstandorts Deutschland zugute. ${ }^{30}$

Dennoch entscheidet sich der Regierungsentwurf für den Ausschluss der Aktie - zumindest vorerst. Die Bundesregierung begründet dies damit, dass die Erstreckung des eWpG auf Aktien „erhebliche gesellschaftsrechtliche Auswirkungen“ hätte. ${ }^{31}$ Dies würde ihrer Ansicht nach u. a. folgende Bereiche betreffen: „die Gründung der Gesellschaft, die Ausgabe von Aktien, die Übertragung der Aktien auf den internationalen Kapitalmärkten, die Einberufung der Hauptversammlung, Kapitalmaßnahmen sowie den Informationsfluss von der Gesellschaft zum Aktionär“.32

Diese Behauptung ist in ihrer Pauschalität jedenfalls für das elektronische Zentralregisterwertpapier unzutreffend. Dessen Einführung würde die Einberufung der Hauptversammlung oder den Informationsfluss zwischen Gesellschaft und Aktionär nicht im Mindesten ändern, denn für diese ist es gleichgültig, ob die Emission durch eine Globalurkunde in physischer Form oder in elektronischer Form repräsentiert ist. ${ }^{33}$ Die Gründung der Gesellschaft, die Übertragung der Aktien und Kapitalmaßnahmen wie eine Kapitalerhöhung würden durch ein elektronisches Zentralregisterwertpapier ebenfalls nicht erschwert. Im Gegenteil würden diese durch die Entmaterialisierung und die damit verbundene Angleichung an andere Rechtsordnungen erleichtert, denn es bedürfte nicht länger der Ausstellung, Einlagerung und permanenten Bewachung eines papiernen Dokuments. Der Umstellungsaufwand für die Ge-

28 Döding/Wentz, Der Referentenentwurf zur Einführung von elektronischen Wertpapieren und Kryptowertpapieren, WM 2020, 2312.

29 Kusserow, Elektronische Schuldverschreibungen und Blockchain-Anleihen im geltenden Recht, WM 2020, 586, 587. Siehe zum Vergleich des deutschen Systems mit anderen Rechtsordnungen Lehmann, Finanzinstrumente (2010), S. $151 \mathrm{ff.}$

30 Ebenso Döding/Wentz, WM 2020, 2312.

31 Bundesregierung, Begründung zum Entwurf eines Gesetzes zur Einführung von elektronischen Wertpapieren (2020), S. 41 (zu $\$ 1$ ).

32 Bundesregierung, Begründung zum Entwurf eines Gesetzes zur Einführung von elektronischen Wertpapieren (2020), S. 41 (zu \1).

33 Ebenso Wieneke/Kunz, Das Gesetz zur Einführung von elektronischen Wertpapieren, NZG 2021, 316, 317. 
sellschaften im Fall der Einführung elektronischer Zentralregisteraktien wäre gleich null; diese würden im Gegenteil sogar die Kosten der Verwahrung der physischen Globalurkunde sparen. Größere Sicherheit als eine digitale Speicherung, welche problemlos vervielfacht werden kann, bietet ein Papier ebenfalls nicht. Der Rechtsverkehr, insbesondere die Aktionäre und die jeweiligen Intermediäre, würde die Umstellung wahrscheinlich nicht einmal bemerken. Diese hätte also nur Vorteile und keinerlei Nachteile. Leider wurde dies von den beteiligten Ministerien verkannt.

Die Einführung elektronischer Kryptoaktien wäre zwar komplizierter, hätte allerdings gewichtige Vorteile, die den Aufwand wert wären. Zum einen würde sie deutsche Unternehmen für „moderne“ Investoren attraktiver machen und damit deren Eigenkapitalbasis stärken. Zum anderen würde sie die Identifikation der Gesellschafter („know your shareholder“) ebenso erleichtern wie die Verfolgung des Schicksals der Gesellschaftsanteile (tracable shares). ${ }^{34}$

Dass die Einführung von Kryptoaktien durchaus im Bereich des Machbaren liegt, zeigt das französische Recht. Dort hat der Gesetzgeber die Ausgabe und den Handel von Aktien auf DLT-Basis mittels sog. DEEP (dispositifs d'enregistrement électronique partagé) durch eine Reihe legislativer und ministerieller Akte ermöglicht. ${ }^{35}$ Dabei hat er zwar börsengehandelte Titel ausgenommen, ${ }^{36}$ dies geschah allerdings nur, um eventuelle Widersprüche mit dem EU-Recht, insbesondere der CSDR, zu vermeiden. ${ }^{37}$ Die Einführung von Kryptoaktien hat die Einberufung von Hauptversammlungen in Frankreich nicht erschwert, sondern ganz im Gegenteil erleichtert. Der Code de commerce schreibt insoweit vor, dass die Aktionäre ihre Teilnahmeberechtigung entweder mittels einer bei der Gesellschaft registrierten Namensaktie oder mittels eines Eintrags auf der Blockchain nachweisen können. ${ }^{38}$ Auch für andere gesellschaftsrechtliche

${ }^{34}$ Möslein/Omlor/Urbach, Grundfragen eines Blockchain-Kapitalgesellschaftsrechts, ZIP 2020, 2149, 2154.

35 Siehe dazu Loi no 2015-990, pour la croissance, l'activité et l'égalité des chances économiques, Journal Officiel vom 7. August 2015, S. 13537, Art. 168; Ordonnance Nr. 2016-520 vom 28. April 2016, relative au bons de caisse, Journal Officiel vom 29. April 2016, Text Nr. 16; Ordonnance Nr. 2017-1674 vom 8. Dezember 2017, relative à l'utilisation d'un dispositif d'enregistrement électronique partagé pour la représentation et la transmission de titres financiers, Journal Officiel, 9. Dezember 2017, Text Nr.24; Decret Nr. 2018-1126 vom 24. Dezember 2018, relatif à l'utilisation d'un dispositif d'enregistrement électronique partagé pour la représentation et la transmission de titres financiers et pour l'émission et la cession de minibons, Journal Officiel vom 26. Dezember 2018, Text Nr. 33. Die meisten Vorschriften dieser Rechtsakte ändern oder ergänzen das französische Währungs- und Finanzgesetzbuch (Code monétaire et financier), siehe dessen Art. L211-3ff.

36 Siehe Art. R211-5 Code monétaire et financier.

37 Siehe Europäische Kommission, Dekret über die Nutzung eines verteilten elektronischen Aufzeichnungssystems zur Darstellung und Übertragung von Wertpapieren, Notifizierungsnummer: 2018/367/F, Eingangsdatum 17. 07. 2018.

38 Art. R225-86 französischer Code de commerce. 
Fragen hat das französische Recht eine einfache Lösung gefunden, indem es die DEEP mit dem herkömmlichen Aktienregister grundsätzlich gleichstellt. ${ }^{39}$

Von einer Reform wie der französischen, welche die Unternehmensfinanzierung mittels Kryptoaktien ermöglicht, ist Deutschland weit entfernt. Selbst die Globalaktie muss noch in Papierform ausgestellt werden. Die Bundesregierung macht allerdings Hoffnung, indem sie verkündet, die Einführung der elektronischen Aktie solle in einem nächsten Schritt erfolgen. ${ }^{40}$ Der Entwurf sei so gefasst, „dass eine spätere Öffnung für elektronische Aktien rechtsetzungstechnisch problemlos erfolgen kann“. ${ }^{4}$

Hierbei wird allerdings übersehen, dass die Inhaberaktie im Bereich des Aktienrechts nur noch eingeschränkt zulässig ist. ${ }^{42} \mathrm{Das} \mathrm{eWpG}$ ist hingegen auf Inhaberpapiere ausgelegt, weil der Gesetzgeber davon ausging, dass ein Bedürfnis des Finanzmarkts für elektronische Orderpapiere nicht gegeben sei. ${ }^{43}$ Dabei handelt es sich um einen Irrtum, denn die Namensaktie - trotz des missverständlichen Namens ein Orderpapier ${ }^{44}$ - ist heute eine häufige Form der Verbriefung, derer sich insbesondere auch bedeutende Aktiengesellschaften bedienen. ${ }^{45}$ Damit das Gesetz den Großteil der Aktien erfassen kann, muss es daher auch auf Orderpapiere erweitert werden.

\section{Anteile an Investmentaktien-und Investmentkommanditgesellschaften}

Anteile an Investmentaktien- und Investmentkommanditgesellschaften können nicht elektronisch begeben werden (siehe oben I.2). Das ist eine weitreichende Einschränkung der Reform. Sie steht in Kontrast zum Anwendungsbereich des ewigen Konkurrenten des deutschen Rechts im Fondsbereich, dem Luxemburger Recht, das eine elektronische Begebung der Globalurkunde zulässt. ${ }^{46}$ Die Gründe für den Ausschluss von Anteilen an Investmentaktien- und Investmentkommanditgesellschaften von den Regelungen des eWpG sind die-

39 Siehe dazu Dondero, La blockchain et le droit des sociétés, Bulletin Joly des Sociétés Nr. 05 2019, S. 40.

40 Bundesregierung, Begründung zum Entwurf eines Gesetzes zur Einführung von elektronischen Wertpapieren (2020), S. 41 (zu $\$ 1$ ).

${ }^{41}$ Bundesregierung, Begründung zum Entwurf eines Gesetzes zur Einführung von elektronischen Wertpapieren (2020), S. 41 (zu $\$ 1$ ).

42 Siehe $\$ 10$ Abs. 1 AktG.

43 Bundesregierung, Begründung zum Entwurf eines Gesetzes zur Einführung von elektronischen Wertpapieren (2020), S. 30 (unter „II. Wesentlicher Inhalt des Entwurfs“).

44 Siehe MüKo/Heider, AktG, Bd. 1 (5. Aufl. 2019), \$10 Rn. 29.

45 Sailer-Coceani, in: Hoffmann-Becking (Hrsg.), MHdB GesR, Bd. 4 (5. Aufl. 2020), $\$ 13$ Aktienarten, Aktiengattungen Rn.3.

46 Art. 2 luxemburgische Loi du 6 avril 2013 relative aux titres dématérialisés, Mémorial A n 71 du 15 avril 2013; siehe auch Prenße/Wöckener/Gillenkirch, Der Gesetzesentwurf zur Einführung elektronischer Wertpapiere - Überblick und Bewertung der zukünftigen Rechtslage mit Blick auf die Rechtsordnungen in Frankreich und Luxemburg, BKR 2020, 551,552 . 
selben wie die für den Ausschluss der elektronischen Aktie (siehe oben II.1). Ebenso wie bei dieser sind sie allerdings auch für Anteile an Investmentaktienund Investmentkommanditgesellschaften nicht stichhaltig.

\section{Zwischenscheine, Bezugsscheine und andere mit Aktien vergleichbare Instrumente}

Ebenfalls außerhalb des Anwendungsbereichs des eWpG liegen mit Aktien vergleichbare Wertpapiere. ${ }^{47}$ Dazu gehören sog. Zwischenscheine, d.h. Anteilsscheine, die den Aktionären vor der Ausgabe der Aktien erteilt werden. ${ }^{48}$ Auch Bezugsscheine, die das Recht auf Erhalt einer jungen Aktie im Fall einer Kapitalerhöhung verbriefen, können nicht nach dem eWpG begeben werden. ${ }^{49}$ Das steht in erheblichem Widerspruch dazu, dass Wandelschuldverschreibungen von dessen Anwendungsbereich erfasst sind, obwohl sie ebenfalls das Recht auf den Erhalt von Aktien verbriefen (siehe oben I.1). Der Ausschluss von Eigenkapitalinstrumenten hat mithin Wertungswidersprüche innerhalb des Gesetzes zur Folge.

\section{Rektapapiere}

Nicht unter das eWpG fallen Rektapapiere, d.h. solche Titel, die nicht durch Übergabe, sondern durch Abtretung übertragen werden. ${ }^{50}$ Dazu gehört zum Beispiel die Namensschuldverschreibung. ${ }^{51}$ Beispiele für Namensschuldverschreibungen sind Kommunalobligationen, Sparbriefe und Sparkassenbriefe, die auf einen bestimmten Berechtigten lauten. Die Ausnahme dieser Titel könnte man damit rechtfertigen, dass es sich bei ihnen nicht um Wertpapiere i.S.d. $\$ 2$ Abs. $1 \mathrm{WpHG}$ handelt ${ }^{52}$ und auch im Zivilrecht ihre Einordnung als Wertpapier nicht ganz zweifelsfrei ist. ${ }^{53}$ Diese dogmatische Überlegung darf jedoch nicht über das praktische Bedürfnis für eine Erstreckung des eWpG auf Rektapapiere hinwegtäuschen. Z. B. könnte eine Übertragung von Sparbriefen auf der Blockchain für die Berechtigten durchaus von Interesse sein.

\footnotetext{
47 Vgl. die in $\$ 2$ Abs. 1 Nr. 2 WpHG genannte Kategorie.

48 Siehe $\$ 8$ Abs. 6 AktG.

49 Siehe zu den Bezugsrechten $\$ 186 \mathrm{AktG}$.

50 Zum Begriff der Rektapapiere siehe Hueck/Canaris, Recht der Wertpapiere (12. Aufl. 1986), S. 21 f.; MüKo/Habersack, BGB, Bd. 7 (8. Aufl. 2020), vor $\$ 793$ Rn. 18; BeckOK/Gehrlein, BGB (57.Ed. 01.02.2021), \$793 Rn. 2.

${ }^{51}$ Franke, Stiefkinder der Jurisprudenz: die Namensschuldverschreibung, DB 1983, 377380; Lebmann, Finanzinstrumente (2010), S. 328.

${ }^{52}$ Siehe zum Ausschluss von Rektapapieren aus dem Wertpapierbegriff des WpHG Schwark/Zimmer/Kumpan, Kapitalmarktrechts-Kommentar (5.Aufl. 2020), $\$ 2$ WpHG Rn. 31 .

${ }^{53}$ Hueck/Canaris, Recht der Wertpapiere (12. Aufl. 1986), S. 6.
} 


\section{Vermögensanlagen}

Definitionsgemäß sind Vermögensanlagen keine Wertpapiere, vgl. \1 Vermögensanlagengesetz (VermAnlG). Daher überrascht es kaum, dass der Gesetzgeber sie nicht in den Anwendungsbereich des eWpG einbezogen hat. Trotzdem könnte ein praktisches Bedürfnis für die Anwendung der Kryptotechnologie auf diese bestehen. Die Blockchain-Strategie der Bundesregierung erwähnt z. B. ausdrücklich die Ausübung von Mitgliedschaftsrechten in einer Genossenschaft als einen möglichen Anwendungsfall von DLT. ${ }^{54}$ Mindestens ebenso praktisch vorteilhaft wäre es, wenn die Mitgliedschaft in einer Genossenschaft oder ein veräußerlich gestellter $\mathrm{GmbH}-$ Geschäftsanteil auf der Blockchain übertragen werden könnte. Der Vorteil der DLT besteht in der Ermöglichung von peer-to-peer-Transfers, die gerade für diese Vermögenswerte eine Rolle spielen.

\section{Derivate}

Ebenfalls ausgeschlossen vom Anwendungsbereich des eWpG sind Derivate. Das ist verständlich, weil Derivate gegenseitige Verpflichtungen enthalten können und sich demzufolge ihre Emission und Abwicklung grundlegend von denjenigen von Wertpapieren unterscheidet. Das hat auch rechtliche Folgen. Beispielsweise unterfallen Derivate nicht dem Depotgesetz. ${ }^{55}$ Ihr Handel erfolgt nicht durch Übertragung, sondern durch Novation. ${ }^{56}$ Ein praktisches Bedürfnis für ein elektronisches Zentralregisterwertpapier für Derivate besteht daher nicht.

Allerdings ist die Grenze zwischen Derivat und Inhaberschuldverschreibung fließend. So sind Optionsscheine nach herrschender Meinung von $\$ 793$ BGB erfasst, obwohl sie regulatorisch als Derivat angesehen werden. ${ }^{57}$ Auf Optionsscheine ist das eWpG anwendbar, denn es folgt wegen seines überwiegend zivilrechtlichen Regelungsgehalts grundsätzlich der zivilrechtlichen Klassifikation und nicht der regulatorischen. ${ }^{58}$ Am Ende ist es daher nur die Verbriefung, die Optionsscheine unter das eWpG fallen lässt, andere Derivate aber nicht. Ein überzeugender rechtspolitischer Grund für diese Ungleichbehandlung ist nicht ersichtlich.

\footnotetext{
54 BT-Drs. 19/13433, S. 14.

55 Müko/Singhof, HGB, Bd. 6 (4. Aufl. 2019), L. Emissionsgeschäft Rn.7.

56 Siehe Brambring, Zentrales Clearing von OTC-Derivaten unter EMIR (2017), S. 157, $248 \mathrm{f}$.

57 MüKo/Habersack, BGB, Bd.7 (8. Aufl. 2020), $\$ 793$ BGB Rn. 10 (Einordnung unter $\$ 793$ BGB); die regulatorische Einordnung ergibt sich daraus, dass Optionsscheine Schuldtitel im Sinne des $\$ 2$ Abs. 1 Nr. 3 lit.b) WpHG und diese gemäß $\$ 2$ Abs. $35 \mathrm{WpHG}$ als Derivate einzuordnen sind, siehe Schwark/Zimmer/Kumpan, Kapitalmarktrechts-Kommentar (5. Aufl. 2020), $\$ 2$ WpHG Rn. 29, 234.

58 Siehe zum Regelungsgehalt des eWpG allgemein Lehmann, NJW 2021, 2318.
} 
Zudem besteht ein praktisches Bedürfnis dafür, Derivate auf der Blockchain zu emittieren und abzuwickeln. Bereits jetzt arbeitet die International Swaps and Derivatives Association (ISDA) mit dem Unternehmen R3 an einem SwapVertrag auf DLT-Basis. ${ }^{59}$ Solche „Kryptoderivate“ könnten signifikante Vorteile bieten, z. B. bei der Abwicklung der gegenseitigen Verpflichtungen über smart contracts. Daher sollte zumindest perspektivisch die Öffnung der Vorschriften des eWpG über Kryptowertpapiere zugunsten von Derivaten geprüft werden.

\section{Entmaterialisierte Werte und Wertpapiere außerhalb des eWpG}

\section{Schuldbuchforderungen}

Das eWpG ist nicht das erste Gesetz, das zu einer Entmaterialisierung führt. Bereits seit dem Jahr 1910 konnten Reichsanleihen als Buchschulden begeben werden. ${ }^{60}$ Diese - durch drei Verordnungen der Nachkriegszeit erweiterte und vertiefte $^{61}$ - Tradition setzt heute das Bundesschuldenwesengesetz (BSchuWG) fort. $^{62}$ Das BSchuWG errichtet ein Bundesschuldbuch, in das Forderungen gegen den Bund und dessen Sondervermögen eingetragen werden können. ${ }^{63}$ Der Gegenstand dieser Forderungen können Schuldverschreibungen, Darlehen, Wechsel, Bankkredite, sonstige an den Finanzmärkten übliche Finanzierungsinstrumente sowie an den Finanzmärkten eingeführte derivative Finanzierungsinstrumente sein, soweit diese haushaltsrechtlich zulässig sind. ${ }^{64} \mathrm{Das}$ Schuldbuch kann auch elektronisch geführt werden; ${ }^{65}$ von dieser Möglichkeit wird heute durchgehend Gebrauch gemacht. Das Schuldbuch des Bundes wird durch die Bundesrepublik Deutschland - Finanzagentur GmbH unter der Aufsicht des Bundesministeriums der Finanzen geführt. ${ }^{66}$ Die Länder haben ent-

59 Siehe https://www.isda.org/2020/02/10/private-international-law-aspects-of-smartderivatives-contracts-utilizing-dlt/.

60 Reichsschuldbuchgesetz vom 31.5.1910, RGBl. I, 840. Dazu Lebmann, Finanzinstrumente (2010), S.37f.

61 Verordnung vom 5.1.1940 über die Verwaltung und Anschaffung von Reichsschuldbuchforderungen, RGBl. 1940 I, S. 30; Verordnung vom 31.12.1940 über die Behandlung von Anleihen des Deutschen Reichs im Bank- und Börsenverkehr, RGBl. 1941 I, S.24; Zweite Verordnung vom 18.4.1942 über die Behandlung von Anleihen des Deutschen Reiches im Bank- und Börsenverkehr, RGBl. 1942 I, S. 183. Zu diesen drei Verordnungen siehe Lehmann, Finanzinstrumente (2010), S. 39-41.

62 Eingeführt durch das Gesetz zur Modernisierung des Schuldenwesens des Bundes (Bundesschuldenwesenmodernisierungsgesetz, BSchuWG) vom 12.7.2006, BGBl. 2006 I, S. 1466, Art. 1. Dazu Lehmann, Finanzinstrumente (2010), S. 43.

63 \5 BSchuWG.

$64 \iint 4 f$. BSchuWG.

65 \4 Abs. 1 S. 2 BSchuWG.

$66 \int 2$ Abs. 1 BSchuWG. 
sprechende Schuldbücher nach dem Vorbild des Bundesschuldbuchs errichtet. ${ }^{67}$ In die Schuldbücher von Bund und Ländern können entweder Sammelbuchforderungen oder Einzelbuchforderungen eingetragen werden. ${ }^{68}$

Mit der Schuldbuchforderung existierte bereits lange vor dem eWpG eine Form des elektronischen Wertpapiers. Der mögliche Einwand, es handele sich dabei nicht um ein „Papier“, sondern lediglich um eine „Forderung“, verkennt, dass auch beim „elektronischen Wertpapier“ keine Urkunde ausgestellt wird. Tatsächlich erfüllt die Schuldbuchforderung im Rechtsverkehr die Funktionen des Wertpapiers, wie Liberations- und Legitimationsfunktion. Der eingetragene Inhaber kann die Eintragung ändern lassen und damit einen Rechtsübergang herbeiführen. ${ }^{69}$ Auch ein gutgläubiger Erwerb ist möglich. ${ }^{70}$ An der Schuldbuchforderung können auch Pfandrechte und ein Nießbrauch bestellt und gutgläubig erworben werden. ${ }^{71}$ Außerdem kann durch die Einbuchung in die Sammelgiroverwahrung ein Mischbestand mit klassischen papiernen Urkunden kreiert werden. ${ }^{72}$ Damit ist überdeutlich, dass die Schuldbuchforderung dieselben Funktionen wie ein elektronisches Wertpapier erfüllt und diesem hinsichtlich seiner Rechtsnatur gleicht.

Leider hatte der Staat den Anwendungsbereich der Schuldbücher nur für seine eigenen Schulden eröffnet. Dem privaten Rechtsverkehr hat er mehr als ein Jahrhundert lang den Vorteil der Entmaterialisierung vorenthalten. Das eWpG holt nur den Rechtszustand auf, der durch das Reichsschuldbuch bereits im Jahr 1910 erreicht wurde. Problematisch ist allerdings, dass es dazu andere Regelungen trifft als das BSchuWG. Auf diese Weise wird die Rechtsspaltung zwischen öffentlichen und privaten Anleihen perpetuiert, ohne dass ein sachlicher Grund dafür ersichtlich ist.

\section{Handelsrechtliche Wertpapiere}

Neben den Schuldbuchforderungen existieren im HGB verschiedene elektronische Formen von Wertpapieren. Das Gesetz zur Reform des Seehandelsrechts führte im Jahr 2013 die Möglichkeit ein, handelsrechtliche Traditionspapiere elektronisch zu begeben. ${ }^{73}$ Dies betrifft den Ladeschein, den Lagerschein und das Konnossement. Insofern sind statt der Ausstellung papierner Dokumente auch elektronische Aufzeichnungen möglich, sofern die Wahrung der Authen-

\footnotetext{
${ }^{67}$ Siehe z.B. Gesetz über das Staatsschuldbuch des Freistaates Bayern (Staatsschuldbuchgesetz) in der Fassung der Bekanntmachung vom 30. März 2003, BayGVBl. 2003, S. 302. Art. 3 des Gesetzes erklärt $\$ \$ 6-8$ BSchuWG für entsprechend anwendbar.

68 Siehe $\$ \$ 6$ f. BSchuWG.

$69 \$ 7$ Abs. 4 BSchuWG.

70 \$9 Abs. 2 BSchuWG.

1 \$9 Abs. 3 BSchuWG.

\$6 Abs. 5 BSchuWG.

73 Gesetz vom 20.4.2013, BGBl. 2013 I, S. 831.
} 
tizität und der Integrität der Aufzeichnung sichergestellt ist. ${ }^{74}$ Die Einzelheiten müssen noch durch eine Rechtsverordnung des Bundesministeriums der Justiz und für Verbraucherschutz festgelegt werden. Diese ist bisher nicht ergangen, so dass die genannten Wertpapiere bislang noch nicht elektronisch begeben werden können. Dennoch sind diese handelsrechtlichen Phänomene ein weiteres Beispiel dafür, dass außerhalb des eWpG bereits elektronische Wertpapiere vorgesehen sind. ${ }^{75}$

Es ist zu erwarten, dass die Ausgestaltung der elektronischen Wertpapiere des HGB von derjenigen des eWpG abweichen wird. Dies folgt bereits daraus, dass die Realität des Handelsrechts grundsätzlich anders ist als die des Kapitalmarktrechts: Ladeschein, Lagerschein und Konnossement werden als individuelle Stücke ausgestellt und übertragen, während Inhaberschuldverschreibungen, Investmentanteilsscheine und Pfandbriefe in großer Zahl begeben und gehandelt werden. Außerdem sind in die Verwahrung der handelsrechtlichen Wertpapiere keine Intermediäre eingeschaltet, während diese bei den kapitalmarktgehandelten Papieren eine überragende Rolle spielen. Die handelsrechtlichen Papiere sind höchst mobil, während die kapitalmarktrechtlichen völlig immobil im Besitz des Intermediärs verbleiben, der diesen an Anleger mittelt. In der Konsequenz fehlt es für eine Sammelverwahrung von Ladeschein, Lagerschein und Konnossement an einem praktischen Bedürfnis. Daher ist für diese zumindest eine Regelung über Zentralregisterwertpapiere nicht nötig. Anders sieht es jedoch mit der Regelung über Kryptowertpapiere aus. Diese sieht einen Transfer von einem Teilnehmer zum anderen (peer to peer) vor, wie er bei handelsrechtlichen Papieren üblich ist. Die entsprechenden Vorschriften des eWpG könnten daher auch insoweit maßstabgebend sein.

\section{Perspektiven einer einheitlichen Regelung elektronischer Wertpapiere}

\section{Integration der verschiedenen Texte in einem Gesetzbuch}

Das derzeitige deutsche Recht der elektronischen Wertpapiere ist formell und materiell zersplittert. Seine Grundlagen sind über verschiedene Gesetzestexte verstreut: eWpG, BSchuWG, Landesschuldbuchgesetze und HGB. Diese folgen zudem keinen einheitlichen Grundsätzen. Zwar ähneln die Vorschriften des eWpG denen über Schuldbuchforderungen in mancherlei Hinsicht, z. B. hinsichtlich der Änderung des Registers oder des gutgläubigen Erwerbs. Sie sind

${ }^{74}$ Siehe $₫ 443$ Abs. 3 HGB, $\$ 475$ Abs. 3 HGB und $₫ 516$ Abs. 2 HGB. Ebenfalls durch das Gesetz zur Reform des Seehandelsrechts eingeführt wurde der elektronische Seefrachtbrief, siehe $\$ 526$ Abs. 4 HGB. Dieser ist jedoch kein Wertpapier und ihm auch nicht gleichgestellt.

75 Siehe Saive, Einführung elektronischer Wertpapiere, ZRP 2020, 219. 
jedoch viel detaillierter als diese und weichen dadurch von den Vorschriften über Schuldbuchforderungen ab. Für die handelsrechtlichen Wertpapiere fehlt es bislang noch an einer Ausgestaltung; es ist jedoch zu erwarten, dass diese weitere Abweichungen von den bereits existierenden Ausprägungen elektronischer Wertpapiere enthalten wird.

Damit kennt das deutsche Recht im Ergebnis drei verschiedene Regime für elektronische Wertpapiere: Kapitalmarktinstrumente, Instrumente der Staatsfinanzierung und handelsrechtliche Instrumente. Dies unterscheidet sich stark von der Situation des klassischen Wertpapierrechts. Letzterem gelang es, die unterschiedlichen Wertpapierarten - trotz der unbezweifelbaren Verschiedenheiten etwa zwischen den handelsrechtlichen Traditionspapieren und der am Kapitalmarkt gehandelten Titel - in einem einheitlichen dogmatischen Rahmen $\mathrm{zu}$ vereinigen. Das neue Recht ist dagegen fragmentiert.

Kann dieser unvorteilhafte Zustand überwunden und die Einheit des Wertpapierrechts auch unter den Bedingungen der Digitalisierung wiederhergestellt werden? Ausgeschlossen ist dies nicht. Die in den verschiedenen Regelungen behandelten Fragen sind weitgehend deckungsgleich. Überall sind etwa Anforderungen an die Registersicherheit, an die Eintragung und deren Änderung oder an den gutgläubigen Erwerb geregelt. Die zugrundeliegenden tatsächlichen Umstände sind ähnlich, teilweise sogar identisch. Eine Schuldbuchforderung gegen den Bund oder eines seiner Sondervermögen hat beispielsweise dieselbe wirtschaftliche Funktion wie eine Inhaberschuldverschreibung gegen einen privaten Emittenten. Es gibt daher keinen überzeugenden Grund dafür, den Erwerb von Schuldbuchforderungen und Inhaberschuldverschreibungen verschiedenen Regelungen zu unterwerfen. Dies verursacht unnötigen gesetzgeberischen Mehraufwand und zusätzliche Rechtsinformations- und Anwendungskosten. Auch bei den handelsrechtlichen Wertpapieren ist die Situation nicht so verschieden, dass sie nicht ebenso geregelt werden könnten wie die kapitalmarktgehandelten Titel. Insbesondere sind die Anforderungen an die Wahrung der Authentizität und die Integrität der Aufzeichnung für beide Kategorien so ähnlich, dass sie einheitlich festgelegt werden können. Auch die Einzelheiten der Ausstellung und Übertragung - einschließlich des gutgläubigen Erwerbs können identisch geregelt werden. Mit einigen Modifikationen können daher die Vorschriften des eWpG über Kryptowertpapiere auf die handelsrechtlichen Wertpapiere übertragen werden. Dafür besteht ein praktisches Bedürfnis, denn verschiedene Anbieter ermöglichen bereits digitale Frachtpapiere mittels Kryptotechnologie; ${ }^{76}$ das Handelsrecht ist sogar eines der Gebiete, für die sich DLT am besten eignet. ${ }^{77}$

${ }^{76}$ Für einen Überblick über die Anbieter entsprechender Lösungen siehe Twenhöven/ Petersen, Impact and Beneficiaries of Blockchain in Logistics, in: Kersten/Blecker/Ringle (Hrsg.), Artificial Intelligence and Digital Transformation in Supply Chain Management (2019), S. 443. 
Perspektivisch sollte man daher überlegen, den Anwendungsbereich des eWpG in der Zukunft auf alle drei Arten von elektronischen Wertpapieren Kapitalmarktinstrumente, Schuldbuchforderungen und handelsrechtliche Papiere - zu erstrecken. Der Unterschied zwischen den ersten beiden Kategorien könnte dabei ganz verschwinden; dieser ist ohnehin lediglich historisch bedingt. Für die handelsrechtlichen Wertpapiere könnten dagegen einige Sonderregelungen gelten. So könnte bei ihnen beispielsweise die Sammeleintragung ausgeschlossen werden. Solche besonderen Vorschriften für einzelne Wertpapierarten gab es auch schon im früheren Recht. Sie stehen der Notwendigkeit und Nützlichkeit einheitlicher Prinzipien und Regelungen nicht entgegen.

\section{Einbeziehung aller Arten von Wertpapieren}

Neben einer Zusammenfassung aller Papiere unter dem Dach des eWpG kommt die Ausdehnung dessen Regeln auf andere Wertpapierarten in Betracht, die bislang nicht elektronisch begeben werden können. An vorderster Stelle stehen dabei Aktien und aktienähnliche Instrumente wie Zwischen- oder Bezugsscheine. Um diese in das eWpG einbeziehen zu können, muss es auf Orderpapiere erweitert werden, denn die meisten Aktien werden heute in der Form der Namensaktie begeben (siehe oben II.1). Dies wäre ein grundlegender Richtungswechsel, denn das Gesetz schließt Orderpapiere noch aus, wie nicht zuletzt auch in der Begründung zum Regierungsentwurf betont wird. ${ }^{78}$

Allerdings dürfte sich der gesetzgeberische Aufwand insoweit in Grenzen halten. Das gilt zunächst für die Einführung von Zentralregisterwertpapieren. Namensaktien konnten schon bisher als Globalurkunden begeben werden, soweit sie ein Blankoindossament enthielten. ${ }^{79}$ Die Ersetzung dieses Globalorderpapiers durch ein elektronisches Wertpapier ändert die Voraussetzungen und Wirkung der Übertragung nicht. Diese funktioniert genau wie bisher durch Einigung und Übergabe. Wie vom Gesetzgeber intendiert, hat die Einführung des Zentralregisterwertpapiers keinen Einfluss auf die Praxis des Effektengiroverkehrs, mit Ausnahme der internen Sphäre des Sammelverwahrers. Daher kann sie ohne grundlegende Änderungen vorgenommen werden. Zur Einführung von Kryptoaktien bedarf es wie gesehen weitergehender Änderungen, z.B. hinsichtlich der Identifikation von Aktionären. Insoweit kann das französische Recht als Vorbild dienen (siehe oben III.1).

77 Takahashi, Blockchain technology and electronic bills of lading, Journal of International Maritime Law 2016, 202.

78 Siehe oben Fn. 43.

${ }^{79}$ MüKo/Bayer, AktG, Bd.1 (5.Aufl. 2019), $\$ 68$ Rn.6; BeckOGK/Cabn, AktG (19.10.2020), $\$ 68$ Rn.7. Für börsengehandelte Aktien ebenso Maul, Zur Ausgabe von Namensaktien, NZG 2001, 585, 586. 


\section{Erweiterung auf andere Gegenstände als Wertpapiere}

Die Digitalisierung ist ebenso wie die Einführung der Kryptotechnologie nicht auf Wertpapiere beschränkt. Einige ihrer vielversprechendsten Anwendungen liegen gerade außerhalb der Wertpapiere. Die Beschränkung der Reform des deutschen Rechts auf Wertpapiere geht daher fehl. ${ }^{80} \mathrm{Um}$ den modernen Erscheinungen Rechnung zu tragen, müsste der Anwendungsbereich des eWpG von Wertpapieren abstrahiert und breiter gefasst werden.

Auf diese Weise könnten z.B. Repräsentationen von Eigentum und anderen Rechten an physischen Gegenständen reguliert werden. Bereits heute wird darüber nachgedacht, Grundstückstransaktionen auf der Blockchain auszuführen. ${ }^{81}$ Dasselbe gilt für Waren, wie z. B. Diamanten, Nutztiere oder Kunstgegenstände, bei denen die Kryptotechnologie zugleich als Herkunftsnachweis und als Eigentumsnachweis dienen kann. ${ }^{82}$ Diese Phänomene liegen bislang außerhalb des Anwendungsbereichs des eWpG. Daneben kommen auch andere Erscheinungen in Betracht, die bislang nicht vom eWpG erfasst sind. Dazu zählen z. B. Kommanditbeteiligungen an Investmentkommanditgesellschaften oder Genossenschaftsanteile. Ebenfalls zählen sog. utility token dazu, die Ansprüche auf eine Leistung repräsentieren. Sie sind eine Art Genussschein der virtuellen Welt. Auch sog. stable coins, die mit Vermögenswerten unterlegt sind, könnten durch das eWpG geregelt werden.

Außerdem erfasst das eWpG seiner Konzeption nach keine Vermögenswerte, die nur in der digitalen Welt existieren und keine Entsprechung in der realen Welt haben. Dazu zählen z. B. Kryptowährungen, andere Onlinegegenstände oder Beteiligungen an sog. Decentralized Autonomous Organizations (DAOs). Diese sog. „native assets“, die keine Existenz außerhalb der Blockchain haben, gehören zu den wirtschaftlich bedeutsamsten Vermögensgegenständen, die mittels der Blockchain übertragen werden.

Dass sie einer rechtlichen Regelung zugänglich sind, zeigt u. a. das liechtensteinische Recht, das für ihre kryptographische Begebung eine Regelung gefunden hat. ${ }^{83}$ Dieses beschreibt seinen Anwendungsbereich ganz allgemein mit „Token“, statt auf Wertpapiere abzustellen. Dies gilt eingeschränkt auch für das Schweizer Recht, das lediglich auf der Blockchain repräsentierte Rechte als „Re-

\footnotetext{
80 Siehe auch Saive, ZRP 2020, 219.

81 Für einen Überblick über entsprechende Initiativen siehe Verbeye, Land Registration in the Twenty-First Century: Blockchain Land Registers from a Civil Law Perspective, in: Lehavi/Levine-Schnur (Hrsg.), Disruptive Technology, Legal Innovation, and the Future of Real Estate (2020), S.123, 124-126; siehe hierzu grundsätzlich auch Simmchen, Grundbuch ex machina (2019).

82 Ein solcher Herkunftsnachweis wurde beispielsweise von dem Unternehmen Everledger realisiert, https://www.everledger.io.

${ }^{83}$ Art. 3 Abs. 3 liechtensteinisches Gesetz vom 3. Oktober 2019 über Token und VTDienstleister (Token- und VT-Dienstleister-Gesetz; TVTG).
} 
gisterwertrechte“ 84 ausdrücklich regelt. ${ }^{85}$ Wenn Deutschland tatsächlich Vorreiter auf dem Gebiet der Blockchaintechnologie werden will, wird es sich auch mit anderen Vermögenswerten als Wertpapieren beschäftigen müssen. Dazu bedarf es allerdings eines entsprechenden politischen Willens.

\section{Die neue Nomenklatur der Wertpapiere}

Doch zurück zu den Wertpapieren. Auch wenn diese nicht in einem Gesetz niedergelegt sind, erscheint es lohnenswert, einen dogmatischen Überblick über sie zu erlangen.

\section{Physische und elektronische Wertpapiere}

Das System der Wertpapiere ist in Deutschland heute zweigeteilt: Auf der einen Seite stehen die traditionellen Wertpapiere in Papierform. Diese können auch weiterhin begeben werden. Anders als etwa in Frankreich, wo kapitalmarktgehandelte Titel schon seit 1983 vollständig entmaterialisiert sind, hat sich der deutsche Gesetzgeber nicht für eine Zwangsentmaterialisierung entschieden. Die Titel existieren in den bekannten Formen, d. h. entsprechend ihrer wirtschaftlichen Funktion als solche des Zahlungs- und Kreditverkehrs, des Effekten- oder Kapitalmarktverkehrs und des Handelsverkehrs. ${ }^{86}$ Auf der anderen Seite stehen die elektronischen Wertpapiere. Als solche können nur Inhaberschuldverschreibungen (einschließlich Pfandbriefe), Staatsanleihen, gewisse Investmentanteile und einige handelsrechtliche Wertpapiere ausgestellt werden. Andere Papiere sind dagegen der elektronischen Form nicht fähig (siehe die Übersicht auf S. 77).

Die grundsätzliche Zweiteilung in physische und elektronische Wertpapiere folgt aus der Art der Begebung. Sie sagt jedoch noch nichts über die Rechtsnatur und die damit zusammenhängende rechtliche Behandlung aus. Eine Zwitterstellung nehmen insoweit die Zentralregisterwertpapiere ein, als sie zwar elektronisch ausgestellt werden, aber qua Fiktion als körperlicher Gegenstand (Sache) gelten ( $\$ 2$ Abs. 3 eWpG). Sie stehen damit mit einem Bein im Recht der elektronischen, mit dem anderen im Recht der physischen Wertpapiere. Kraft dieser Doppelstellung sollen sie die beiden Welten miteinander verbinden. Umso weniger verständlich ist es, dass z. B. die Aktie nicht auch elektronisch ausgestellt werden kann.

84 Art. 973d Abs. 1 OR.

85 Enz, Die zivilrechtliche Einordnung von Zahlungs-Token wie dem Bitcoin als „Registerwertdaten “ und deren Aussonderbarkeit im Konkurs de lege lata und de lege ferenda, SJZ 2020, 291, $295 \mathrm{f}$.

${ }^{86}$ Hueck/Canaris, Recht der Wertpapiere (12. Aufl. 1986), S. 19 f. 


\begin{tabular}{lll} 
& physische Wertpapiere & elektronische Wertpapiere \\
\hline $\begin{array}{l}\text { Papiere des Kredit- } \\
\text { und Zahlungsverkehrs }\end{array}$ & $\checkmark$ & - \\
$\begin{array}{l}\text { Scheck, Wechsel) } \\
\text { Papiere des Kapital- } \\
\text { markts (Aktie, Anleihe, }\end{array}$ & $\checkmark$ & \\
Derivate) & - Inhaberschuldverschreibungen \\
& (einschließlich Staatsanleihen \\
& und andere Schuldbuchforde- \\
& rungen) \\
& - Pfandbriefe \\
Papiere des Handels- & $\checkmark$ & - Investmentanteilsscheine \\
verkehrs & & - Lagerschein \\
& & - Ladeschein \\
& & - Konnossement \\
\hline
\end{tabular}

Im herkömmlichen Recht ist zudem die Unterscheidung zwischen Inhaber-, Order- und Rektapapieren bekannt. ${ }^{87}$ Fraglich ist, ob sich diese auch auf die elektronischen Wertpapiere übertragen lässt. Dies kommt insbesondere für die drei elektronischen Wertpapiere des Handelsrechts in Betracht, die nichts anderes sind als das Spiegelbild der papiernen Varianten. Ladeschein, Lagerschein und Konnossement sind „gekorene Orderpapiere“.:88 Sie können nach $\$ 363$ Abs. 2 HGB als Orderpapiere ausgestellt werden; ansonsten sind sie Inhaberpapiere. Es ist noch offen, ob und wie die ausstehende Rechtsverordnung die Unterscheidung zwischen Order- und Inhaberpapieren auf die elektronischen Varianten des Ladescheins, Lagerscheins und Konnossements übertragen wird.

Bei den elektronischen Wertpapieren des Kapitalmarkts - Schuldbuchforderungen, elektronische Zentralregisterwertpapiere und Kryptowertpapiere - ist die Situation komplizierter, denn bei ihnen wird die Unterscheidung in Inhaber-, Order- und Rektapapiere durch eine andere Differenzierung überlagert: die in Sammel- und Einzeleintragung. Diese muss zuerst dargestellt werden (siehe unter 2.), bevor zur Übertragung der klassischen Nomenklatur in Inhaber-, Order- und Rektapapiere Stellung genommen werden kann (siehe unter 3.).

${ }^{87}$ Hueck/Canaris, Recht der Wertpapiere (12. Aufl. 1986), S. 21-25; Sedatis, Einführung in das Wertpapierrecht (1988), Rn. 86-94; MüKo/Habersack, BGB, Bd.7 (8. Aufl. 2020), Vor $\$ 793$ BGB Rn. 15; Langenbucher/Bliesener/Spindler/Haertlein, Bankrechts-Kommentar (3. Aufl. 2020), 28. Kapitel Rn. 12-31; Lehmann, Grundriss des Bank- und Kapitalmarktrechts (2016), Rn. 319-322; Holzhammer, Allgemeines Handelsrecht und Wertpapierrecht (8. Aufl. 1998), S. 286f.; Koller/Wolkerstorfer, Wertpapierrecht (2020), S.12f.

88 Siehe Hueck/Canaris, Recht der Wertpapiere (12. Aufl. 1986), S. 193. 


\section{Sammel-und Einzeleintragung}

Im Gegensatz zu den handelsrechtlichen e-Wertpapieren werden die elektronischen Wertpapiere des Kapitalmarkts (Schuldbuchforderungen, Inhaberschuldverschreibungen [einschließlich Pfandbriefe] und Investmentanteile) massenhaft ausgestellt. Von primärer Bedeutung ist daher die Frage, in welcher Form die Berechtigung aus dem Wertpapier registriert wird. Zwei Arten kommen in Betracht: die Sammeleintragung und die Einzeleintragung. Beide Formen der Eintragung werden durch das eWpG neu eingeführt; sie sind der Sammelschuldbuchforderung und der Einzelschuldbuchforderung des BSchuWG nachgebildet. ${ }^{89}$ Sie unterscheiden sich in folgender Weise: Bei der Sammeleintragung erlangen die Berechtigten einen Miteigentumsanteil am Sammelbestand, der von einem Intermediär verwahrt oder verwaltet wird. ${ }^{90}$ Bei der Einzeleintragung erlangt jeder Anleger individuelles Eigentum am Wertpapier. Die Unterscheidung spielt insbesondere dann praktisch eine Rolle, wenn bei der Sammeleintragung die zum Sammelbestand gehörenden Wertpapiere nicht ausreichen, um die Vermögensrechte der Anleger abzudecken. In diesem Fall wird der Verlust von allen Anlegern pro rata getragen; für den Ausfall haftet der Sammelverwahrer. ${ }^{91}$ Die Einzeleintragung gewährt dem Anleger hingegen einen größeren Schutz. Ein Verlust ist unmöglich, da er ein Eigentumsrecht auch gegenüber Dritten geltend machen kann.

Nach dem eWpG ist bei allen erfassten Instrumenten sowohl eine Sammel- als auch eine Einzeleintragung zulässig. Die Möglichkeit der Einzeleintragung bei Zentralregisterwertpapieren war im Referentenentwurf des eWpG noch nicht vorgesehen ${ }^{92}$ und ist erst durch den Regierungsentwurf aufgekommen. Einzutragen sind neben dem Inhaber auch eventuelle Verfügungshindernisse und Rechte Dritter. ${ }^{93}$ Die Eintragung im Zentralregister soll der Begründung des Regierungsentwurfs zufolge „einen weitergehenden Schutz des Rechtsverkehrs ermöglichen, als sie aus der Publizität der Urkunde als Bezugsobjekt folgt“. Insbesondere soll die Zurechenbarkeit des Rechtsscheins und der damit verbundene Einwendungsausschluss weiter reichen als bei körperlichen Urkunden. ${ }^{94}$

Allerdings ist fraglich, ob dieses Ziel tatsächlich erreicht wird, da eine Bestimmung über den gutgläubigen Erwerb von im Zentralregister eingetragenen

89 Bundesregierung, Begründung zum Entwurf eines Gesetzes zur Einführung von elektronischen Wertpapieren (2020), S. 55.

90 Siehe $\$ 6$ DepotG, $\$ 9$ Abs. 1 S. 2 eWpG.

$91 \$ 7$ Abs. 2 DepotG. Die Vorschrift gilt gemäß $₫ 9$ Abs. 1 S. 1 eWpG auch für elektronische Wertpapiere in Sammeleintragung.

92 Siehe insoweit noch die Beschränkung auf Kryptowertpapiere in $\$ 8$ Abs. 2 eWpG-RefE sowie RefE S. 48f. Kritisch dazu Kleinert/Mayer, Der deutsche Weg zum elektronischen Wertpapier, EuZW, 2020, 1059, 1061.

${ }_{93} \int 13$ Abs. 1 Nr. 6, Abs. 2 eWpG.

94 Bundesregierung, Begründung zum Entwurf eines Gesetzes zur Einführung von elektronischen Wertpapieren (2020), S. 62. 
Wertpapieren gerade fehlt. Die Folge einer unrichtigen Eintragung ist allein die Beseitigungspflicht, und auch diese ist nur auf den Fall der falschen rechtsgeschäftlichen Änderung, nicht auf den unrichtiger Verfügungsbeschränkungen bezogen. ${ }^{95}$ Der lastenfreie rechtsgeschäftliche Erwerb vom Berechtigten ist anders als etwa bei Kryptowertpapieren - gerade nicht vorgesehen. Insofern fehlt dem durch einen Verwahrer geführten Zentralregister eine wichtige Eigenschaft des Wertpapiers - die Übertragung im Fall guten Glaubens.

Anders ist die Situation für Zentralregister, die in den Effektengiroverkehr einbezogen sind. Für diese gelten die Vorschriften über körperlich ausgestellte Wertpapiere, also die $\iint 929 \mathrm{ff}$. BGB und das DepotG. Folglich finden auf sie auch die Vorschriften über den gutgläubigen Erwerb an beweglichen Sachen in $\iint 932 \mathrm{ff}$. BGB Anwendung. Eine Einbeziehung des Zentralregisters in den Effektengiroverkehr ist allerdings nur möglich, soweit das Register durch eine Wertpapiersammelbank ${ }^{96}$ geführt wird und diese als Inhaberin des Zentralregisterwertpapiers eingetragen ist. ${ }^{97}$

Der gutgläubige Erwerb ist damit nur bei von Verwahrern ${ }^{98}$ geführten Registern sowie bei solchen von Wertpapiersammelbanken geführten Registern ausgeschlossen, bei denen eine andere Person als die Wertpapiersammelbank als Inhaber eingetragen ist. Als praktischen Anwendungsfall für von Verwahrern geführte Register nennt die Kreditwirtschaft die „Platzierung von Anleihen bei (institutionellen) Investoren, die eine Übertragung der Wertpapiere nicht beabsichtigen, sondern die Wertpapiere bis zur Endfälligkeit halten wollen".99 In dieser Konstellation ist ein Schutz durch gutgläubigen Erwerb in der Tat verzichtbar. Der Nutzen der Digitalisierung besteht hier allein in der Ersparung der Ausstellung und Aufbewahrung papierner Urkunden, nicht in der Übertragung im Verkehr. Soweit eine Wertpapiersammelbank das Register führt und nicht selbst eingetragen ist, ist die Situation ähnlich.

Auffällig bleibt dennoch, dass das Gesetz einer Einzeleintragung bei Kryptowertpapieren volle wertpapierrechtliche Wirkungen zukommen lässt, inklusive der Möglichkeit eines gutgläubigen Erwerbs, ${ }^{100}$ während der Buchung bei Zentralregisterwertpapieren keine wertpapierrechtliche Bedeutung zukommt. Das gilt insbesondere für die Positionen der Einzelanleger im Effektengiroverkehr, die sich allein auf den Besitzmittlungswillen der Wertpapiersammelbank verlassen müssen, während die Buchung in den Computern der Depotbanken nach $\iint 929 \mathrm{ff}$. BGB keine rechtliche Bedeutung hat. Dies ist in der Literatur

95 Siehe $\$ 14$ Abs. 5 eWpG.

96 Siehe die Definition in $₫ 4$ Abs. 5 eWpG.

$97 \$ 12$ Abs. 3 eWpG.

98 Siehe die Definition in $₫ 4$ Abs. 6 eWpG.

99 Bundesverband der Deutschen Volks- und Raiffeisenbanken e. V., Stellungnahme Regierungsentwurf eines Gesetzes zur Einführung von elektronischen Wertpapieren, 10.3.2021, S. 11 .

100 Siehe $\$ 26$ eWpG. 
stark kritisiert worden mit der Begründung, dass Einzelemissionen nicht allein der Kryptotechnologie vorbehalten sein könnten. ${ }^{101}$ Die Bevorzugung der Kryptotechnologie gegenüber anderen Technologien, etwa der zentralen elektronischen Eintragung, ist in der Tat bedenklich. Sie widerspricht dem Grundsatz der technologischen Neutralität, einem selbsterklärten Ziel des eWpG. ${ }^{102}$ Zudem ist der Begriff der Kryptotechnologie sehr unbestimmt und von der rein elektronischen Form kaum abzugrenzen. Außerdem bietet erstere nicht notwendig mehr Sicherheit als letztere.

Wollte man allerdings der Einzeleintragung auch bei in den Effektengiroverkehr einbezogenen Zentralregistern wertpapierrechtliche Wirkungen zukommen lassen, bedürfte es einer grundsätzlichen Umstellung der Konzeption der Gesetzgebung. Insbesondere müsste auch im allgemeinen Effektengiroverkehr der Buchung die entscheidende rechtliche Bedeutung zukommen, so wie es bei Kryptowertpapieren vorgesehen ist. Das Konzept der Gleichstellung mit körperlichen Urkunden ( $\$ 2$ Abs. $3 \mathrm{eWpG}$ ) und die darauf aufbauende Übertragung mittels Einigung und Übergabe ( $\$ 929$ S. 1 BGB) sind damit unvereinbar. Der Effektengiroverkehr müsste auf ein rein digitales Fundament gestellt werden. $\mathrm{Zu}$ einer solch grundlegenden Reform konnte sich der Gesetzgeber bisher nicht entschließen. Daher sieht die Situation der Sammel- und Einzeleintragung derzeit folgendermaßen aus:

\begin{tabular}{lll} 
& Sammeleintragung & Einzeleintragung \\
\hline Schuldbuchforderungen & $\checkmark$ & $\checkmark$ \\
Zentralregisterwertpapiere & $\checkmark$ & $\checkmark$, aber nicht für individuelle \\
& & Anleger im Effektengiroverkehr \\
Kryptowertpapiere & $\checkmark$ & $\checkmark$ \\
\hline
\end{tabular}

\section{3. Übertragung der herkömmlichen Nomenklatur} auf Zentralregister- und Kryptowertpapiere?

Nach diesem Exkurs kann abschließend Stellung zur Frage genommen werden, ob sich die Einteilung in Inhaber-, Order- und Rektapapiere auf die neuen elektronischen Wertpapiere des Kapitalmarkts übertragen lässt. Die Antwort auf diese Frage folgt aus der Unterscheidung nach Sammel- und Einzeleintragung.

Soweit eine Sammeleintragung vorgenommen wird, ist dem eWpG zufolge als Inhaber stets die Wertpapiersammelbank oder der Verwahrer - d.h. ein Kreditinstitut - eingetragen. ${ }^{103}$ Diese verwalten „die Sammeleintragung“

101 Linardatos, Elektronische Schuldverschreibungen auf den Inhaber - des Wertpapiers neue Kleider, ZBB 2020, 329, 336.

102 Siehe dazu Bundesregierung, Begründung zum Entwurf eines Gesetzes zur Einführung von elektronischen Wertpapieren (2020), S. 1, 30, 68.

$103 \$ 8$ Abs. 1 Nr. 1 eWpG. 
treuhänderisch für die Berechtigten, ohne selbst Berechtigte zu sein. ${ }^{104}$ Damit fallen insoweit Inhaberschaft des Wertpapiers und Berechtigung am Recht aus dem Wertpapier auseinander. Das steht zwar im Einklang mit der Differenzierung zwischen beiden in $₫ 3$ Abs. 1 und $2 \mathrm{eWpG}$, ist jedoch für das Wertpapierrecht eine völlig atypische Situation. Die wirtschaftliche und rechtliche Funktion des Wertpapiers besteht gerade darin, dass das in ihm verbriefte Recht dem Recht am Wertpapier folgt, welches auf diese Weise umlauffähig gemacht wird. ${ }^{105}$ Die bei elektronischen Zentralregisterwertpapieren und Kryptowertpapieren mögliche Sammeleintragung ist demgegenüber eine eigenartige Neuschöpfung, bei der es zwar einen namentlich benannten Inhaber gibt, dieser jedoch gerade keine wertpapierrechtliche Berechtigung innehat. Dies gleicht keiner der bisherigen Wertpapierarten. Man kann insoweit von einem „Treuhänderpapier“ sprechen, bei dem nicht der Inhaber, sondern ein Dritter - der Treugeber - Berechtigter ist. In Wahrheit fehlt diesem allerdings bereits die wesentlichste aller Wertpapierfunktionen, nämlich den Berechtigten zu identifizieren.

Soweit eine Einzeleintragung vorgenommen wird, ähnelt die Situation dagegen zumindest auf den ersten Blick sehr stark derjenigen bei Orderpapieren. Die bloße Inhaberschaft genügt nicht, vielmehr ist eine Nennung des Inhabers notwendig (vgl. \13 Abs. 1 Nr.6, 17 Abs. 1 Nr.6 eWpG). Eine Änderung des Registerinhalts ist grundsätzlich nur mit expliziter Zustimmung oder aufgrund einer Weisung des Berechtigten möglich (\$14 Abs. 1 S. 1, $\mathbb{1} 18$ Abs. 1 S. 1 Nr. 1 eWpG). Dies hat zumindest gewisse Ähnlichkeit mit der bei Orderpapieren nötigen Kette von Indossamenten.

Allerdings hat bei Zentralregisterwertpapieren die Einzeleintragung wie erörtert gerade keine Gutglaubenswirkung. ${ }^{106}$ Insoweit sind diese eher mit den herkömmlichen Rektapapieren vergleichbar.

Bei Kryptowertpapieren ist eine Gutglaubenswirkung zwar vorgesehen $(\mathbb{2} 26$ eWpG). Jedoch kommt diesen insoweit eine Sonderstellung zu, als bei ihnen die Identität des Inhabers gerade nicht öffentlich bekannt ist. Vielmehr muss die Bezeichnung des Inhabers durch Zuordnung einer eindeutigen Kennung erfolgen ( $\$ 17$ Abs. 2 S. $2 \mathrm{eWpG}$ ). Die Identität des Inhabers kann erst durch besondere Auskunft und nur bei Vorliegen bestimmter Voraussetzungen geklärt werden ( $(10$ Abs. $3 \mathrm{eWpG})$. Insofern kann man von einem „verschlüsselten Orderpapier" sprechen.

Daraus ergibt sich folgende neue Nomenklatur der Wertpapiere:

104 \$9 Abs. 2 S. 1 eWpG.

105 Siehe Hueck/Canaris, Recht der Wertpapiere (12. Aufl. 1986), S. 8 ff.; Lehmann, Finanzinstrumente (2010), S.12f., 309; Holzhammer, Allgemeines Handelsrecht und Wertpapierrecht (8. Aufl. 1998), S. 286.

106 Siehe oben VI.2. 


\begin{tabular}{ll} 
physische Wertpapiere & elektronische Wertpapiere \\
\hline Inhaberpapier & $\begin{array}{l}\text { Treuhänderpapier (Sammeleintragung) } \\
\text { Orderpapier }\end{array}$ \\
$\begin{array}{l}\text { Orderpapier mit verschlüsselter Identität (Kryptowertpapier in } \\
\text { Einzeleintragung) } \\
\text { Rektapapier }\end{array}$ & $\begin{array}{l}\text { Wertpapier ohne gutgläubigen Erwerb (Zentralregisterwert- } \\
\text { papier in Einzeleintragung) }\end{array}$ \\
\hline
\end{tabular}

Die elektronischen Wertpapiere haben damit viele der Funktionen ihrer Pendants in der körperlichen Welt eingebüßt. Treuhänderpapiere ohne eine Vermutung der Berechtigung sind weitgehend funktionslos. Orderpapiere ohne volle Transparenz des Berechtigten können mit den klassischen Orderpapieren wie Wechsel, Scheck oder Namensaktie nur mit Vorbehalten verglichen werden. Und Zentralregisterwertpapiere ohne die Möglichkeit eines gutgläubigen Erwerbs sind des Umlaufs kaum fähig.

Daher bedarf es einer grundsätzlichen Neubestimmung des Zwecks des Wertpapiers. Dieser kann unter den Bedingungen der Digitalisierung nicht länger in der Förderung der Umlauffähigkeit durch die Ausstellung von $\mathrm{Pa}-$ pieren gesehen werden. Vielmehr liegt die Funktion der Register allein in den darin enthaltenen Buchungen. Vom Abstellen auf das Wertpapier sollte daher gänzlich abgesehen werden.

\section{Zusammenfassung}

Die Entmaterialisierung des deutschen Wertpapierrechts schreitet voran. Gleichwohl ist das eWpG nicht der erhoffte Riesensatz, sondern eher ein Trippelschritt, wenngleich in die richtige Richtung. Die erhebliche Beschränkung des Anwendungsbereichs und der Ausschluss praktisch besonders relevanter Wertpapierarten - wie etwa der Aktie - sind dabei aus zwei Gründen bedenklich: Zum einen büßt das Gesetz erheblich an Praxisrelevanz ein und wird dementsprechend wohl nur gedämpft ein Echo in der Wirtschaft erzeugen. Zum anderen hat der Gesetzgeber es mit der Schaffung des eWpG versäumt, die Systematik der Wertpapiere einheitlich zu normieren und so ein übersichtliches und kohärentes Regelungsregime zu schaffen. Das deutsche Wertpapierrecht ist heute fragmentiert und voll von inneren Widersprüchen. Es bedarf zu seiner Reform eines mutigeren Ansatzes, der endlich von der längst überholten Vorstellung eines Papiers abstrahiert und rechtliche Folgen allein an elektronische Buchungen anknüpft. 


\title{
$\int 4$ \\ Zentrales Register und Kryptowertpapierregister
}

\author{
Mathias Habersack
}

I. Sachenrechtliche Verankerung des geltenden Wertpapierrechts $\ldots \ldots \ldots \ldots 83$

II. BuSchWG als Inspirationsquelle des eWpG $\ldots \ldots \ldots \ldots \ldots \ldots \ldots \ldots . \quad 85$

1. Sammel- und Einzelschuldbuchforderungen nach dem BuSchWG .... 85

2. Sammel- und Einzeleintragung nach dem eWpG ............ 86

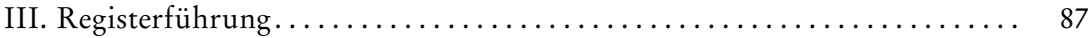

1. Wertpapierregister im Allgemeinen ...................... 87

a) Pflichten und Haftung des Registerführers ................ 87

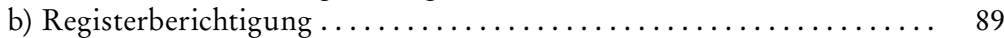

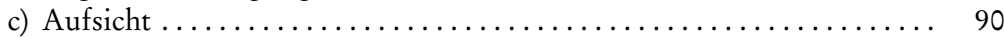

2. Kryptowertpapierregister im Besonderen $\ldots \ldots \ldots \ldots \ldots \ldots \ldots \ldots . \ldots 90$

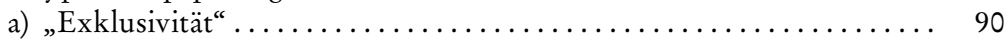

b) Sicherung der Funktionstüchtigkeit des Kryptowertpapierregisters .. 90

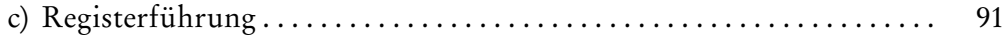

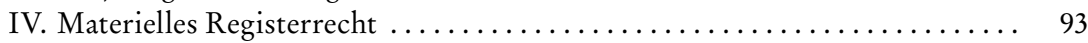

1. Begebung des elektronischen Wertpapiers .................. 93

a) Eintragung und Begebungsvertrag $\ldots \ldots \ldots \ldots \ldots \ldots \ldots \ldots \ldots \ldots$

b) Niederlegung der Emissionsbedingungen $\ldots \ldots \ldots \ldots \ldots \ldots \ldots . \ldots 3$

2. Formelle Legitimation und materielle Berechtigung $\ldots \ldots \ldots \ldots \ldots .94$

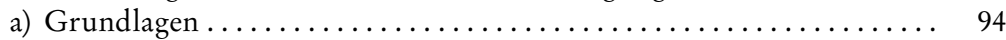

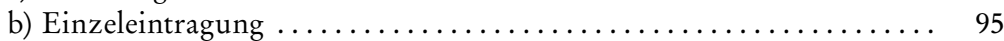

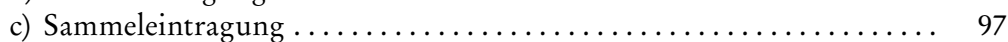

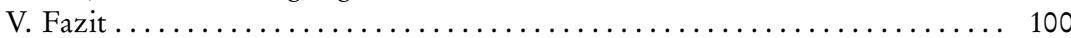

\section{Sachenrechtliche Verankerung des geltenden Wertpapierrechts}

Das geltende Wertpapierrecht befindet sich in keinem guten Zustand: Auf der einen Seite ist der ursprüngliche Zweck des Wertpapiers - die Verkörperung eines privaten Rechts in einer Urkunde, die sodann Gegenstand von Verfügungen sein kann und die den Besitzer als Berechtigten legitimiert - infolge des für die Wertpapiere des Kapitalmarkts praktizierten stückelosen Effektengiroverkehrs mehr oder weniger gegenstandslos geworden; die Übertragung des Papiers wird, dem bargeldlosen Zahlungsverkehr vergleichbar, de facto durch 
Buchungsvorgänge ersetzt. ${ }^{1}$ Auf der anderen Seite ist die ganz herrschende Meinung bestrebt, dem Depotkunden ungeachtet der den Kapitalmarkt kennzeichnenden Entmaterialisierung und Immobilisierung Miteigentum und Mitbesitz am hinterlegten Sammelbestand zuzusprechen, um hierdurch Verfügungen über diese Position unter $\int \mathbb{S} 929 \mathrm{ff}$., $932 \mathrm{ff}$., 1032, $1205 \mathrm{ff}$. BGB zu subsumieren und damit dem ursprünglichen Gedanken des Wertpapierrechts - nämlich der Anknüpfung an die Papierposition - gerecht zu werden. ${ }^{2}$ Besonders deutlich kommt das Bestreben des historischen, durch die „Welt der Einzelurkunde“ geprägten Gesetzgebers, die Verkehrsfähigkeit des Wertpapiers zu gewährleisten, in $\$ 935$ Abs. 2 BGB zum Ausdruck, ermöglicht die Vorschrift doch sogar den gutgläubigen Erwerb des dem Berechtigten abhandengekommenen Inhaberpapiers. Auf eine harte und wohl kaum zu bestehende Belastungsprobe kommt das sachenrechtlich geprägte Modell allerdings spätestens im Zusammenhang mit der heute verbreiteten Globalurkunde, bei der sich mit Blick auf den in $\$ 9$ a Abs. 3 S. 2 DepotG geregelten Ausschluss jeglichen Herausgabeanspruchs eine irgendwie geartete Besitzposition des Depotkunden entgegen der herrschenden Meinung ${ }^{3}$ nicht mehr bejahen lässt. ${ }^{4}$

${ }^{1}$ Zu dieser Entwicklung MüKo/Habersack, BGB (8. Aufl. 2020), Vor $\$ 793$ BGB Rn. 32 ff., Baumbach/Hefermehl/Casper, Wechselgesetz, Scheckgesetz, Recht des Zahlungsverkehrs (24. Aufl. 2020), WPR Rn. 93 ff., 103 ff., jew. m. w. N.; monografisch Einsele, Wertpapierrecht als Schuldrecht - Funktionsverlust von Effektenurkunden im internationalen Rechtsverkehr (1995), S. $172 \mathrm{ff} ., 561 \mathrm{ff}$.; Lehmann, Finanzinstrumente - Vom Wertpapier- und Sachenrecht zum Recht der unkörperlichen Gegenstände (2009), S. 228 ff., 281 ff.; Segna, Bucheffekten (2018), passim, insbes. S. $151 \mathrm{ff}$., 599ff.; jüngst Scherer, Blockchain im Wertpapierbereich (2020), S. $9 \mathrm{ff} ., 50 \mathrm{ff}$.

2 Für die Girosammelverwahrung BGH, Beschl.v. 16.07.2004, Az. IXa ZB 24/04 = BGHZ 160, 121, 124, 126; BGH, Urt. v. 04.02.1999, Az. III ZR 56-98 = NJW 1999, 1393; BGH, Urt. v. 22.04.1997, Az. XI ZR 127/96 = NJW 1997, 2110, 2111; BGH, Urt. v. 24.09.2015, Az. IX ZR 272/13 = ZIP 2015, 2286 Rn. 15ff.; näher Habersack/Mayer, Globalverbriefte Aktien als Gegenstand sachenrechtlicher Verfügungen? - Ein (weiteres) Plädoyer für die Ablösung der Globalurkunde durch Wertrechte-, WM 2000, 1678, 1679f.; Habersack/Ehrl, Börsengeschäfte unter Einbeziehung eines zentralen Kontrahenten, ZfPW 2015, 312, 341 ff.; Segna (Fn. 1), S. $222 \mathrm{ff}$.

${ }^{3}$ BGH, Beschl. v. 16.07.2004, Az. IXa ZB 24/04 = BGHZ 160, 121, 124, 126; BGH, Urt. v. 14.05.2013, Az. XI ZR 160/12 = NZG 2013, 903 Rn. 14; BGH, Urt. v. 24.09.2015, Az. IX ZR 272/13 = ZIP 2015, 2286 Rn. 15ff. m. w. N.; OLG München, Urt. v. 17.01.2018, Az. 7 U 1801/17 = BeckRS 2018, 132 Rn. 36ff.; so oder zumindest im Ergebnis auch Großkommentar zum HGB/Canaris (3. Aufl. 1981), Bankvertragsrecht Rn. 2007 ff.; Horn, Die Erfüllung von Wertpapiergeschäften unter Einbeziehung eines Zentralen Kontrahenten an der Börse - Sachenrechtliche Aspekte -, WM-Sonderbeil. Nr. 2/2002, 14ff.; Hirte/Knof, Das Pfandrecht an globalverbrieften Aktien in der Insolvenz, WM 2008, 7 ff., 49 ff.; Koller, Der gutgläubige Erwerb von Sammeldepotanteilen an Wertpapieren, DB 1972, 1905, 1909; Mülbert, Die Aktie zwischen mitgliedschafts- und wertpapierrechtlichen Vorstellungen, in: FS Nobbe (2009), S. 691, 699; Mohamed, Die Legitimationszession im Aktienrecht (2018), S. $119 \mathrm{ff}$.; S. Schwarz, Globaler Effektenhandel (2016), S. 187 ff.; Segna (Fn. 1), S. $222 \mathrm{ff}$.

${ }^{4}$ Einsele (Fn. 1), S. $172 \mathrm{ff} ., 561 \mathrm{ff}$; Einsele, Wertpapiere im elektronischen Bankgeschäft, WM 2001, 7, 13; MüKo/Habersack (Fn.1), Vor $\$ 793$ BGB Rn. 36; Habersack/Mayer, WM 2000, 1678, 1680ff.; Habersack/Ehrl, ZfPW 2015, 312, 344 ff.; Baumbach/Hefermehl/Cas- 


\section{BuSchWG als Inspirationsquelle des eWpG}

\section{Sammel- und Einzelschuldbuchforderungen nach dem BuScbWG}

Der deutsche Gesetzgeber konnte sich bislang nicht dazu durchringen, den sachenrechtlich geprägten Ansatz des geltenden Wertpapierrechts aufzugeben und durch ein System von Bucheffekten oder Wertrechten zu ersetzen. ${ }^{5} \mathrm{Al}-$ lein für die Begebung von öffentlichen Anleihen hat er schon früh das Modell der Inhaberschuldverschreibung um das der Schuldbuchforderung ergänzt und damit die Möglichkeit geschaffen, die Verbriefung durch einen Registereintrag zu ersetzen: Durch das BWpVerwG ${ }^{6}$ war das bis dahin in mehreren vorkonstitutionellen Gesetzen und Rechtsverordnungen geregelte Schuldbuchrecht auf eine einheitliche rechtliche Grundlage gestellt worden. An die Stelle des BWpVerwG ist sodann am 1.8.2006 das BSchuWG getreten, ${ }^{7}$ nach dessen $\$ 5$ Abs. 3 eine Schuldbuchforderung als Sammelschuldbuchforderung ( $\$ 6$ BSchuWG) oder Einzelschuldbuchforderung ( $\$ 7$ BSchuWG) durch Eintragung in die jeweilige Abteilung ( $\$ 5$ Abs. 2 BSchuWG) begründet wird; unberührt bleibt jeweils das Erfordernis eines Begebungsvertrags. ${ }^{8}$

Was zunächst Sammelschuldbuchforderungen anbelangt, so wird nach $\$ 6$ Abs. 1 BSchuWG in das Schuldbuch eine Wertpapiersammelbank als (nach $\$ 185$ Abs. 1 BGB ermächtigte) Treuhänderin für die gesamte Emisson eintragen. Vor dem Hintergrund, dass die gesetzliche Regelung der Girosammelver-

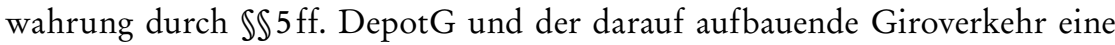
Verbriefung gerade voraussetzen, war es sodann erforderlich, die Sammelschuldbuchforderung in $\$ 6$ Abs. 2 S. 1 BSchuWG im Wege einer gesetzlichen Fiktion mit dem Wertpapiersammelbestand der Wertpapiersammelbank im Sinne des Depotgesetzes gleichzustellen und in $\$ 6$ Abs. 2 S. 2 BSchuWG zu bestimmen, dass die Gläubiger der Sammelschuldbuchforderung als Miteigentümer nach Bruchteilen gelten. Über diese Fiktion erfolgt, wie es in den Materialien anschaulich verlautbart wird, gleichsam eine "Verdinglichung" der an sich nur nach Zessionsrecht übertragbaren Forderung, so dass über die demateria-

per (Fn. 1), WPR Rn. 103 ff.; Casper, Elektronische Schuldverschreibung: es ist Zeit für einen grundlegenden gesetzlichen Neustart, BKR 2019, 209, 210ff.; BeckOGK/Vogel, BGB (Stand 01.01.2021), \$793 BGB Rn. 65: „bloße Fiktion“.

${ }^{5} \mathrm{Zu}$ entsprechenden Überlegungen s. die Nachw. in Fn. 1, ferner Casper, BKR 2019, 209, $212 \mathrm{ff}$.

${ }^{6}$ Gesetz zur Neuordnung des Schuldbuchrechts des Bundes und der Rechtsgrundlagen der Bundesschuldenverwaltung vom 11.12.2001, BGBl. I 2001, S. 3519.

7 Gesetz zur Regelung des Schuldenwesens des Bundes vom 12.7.2006, BGBl. I 2006, S. 1466; dazu BGH, Urt. v. 24.09.2015, Az. IX ZR 272/13 = ZIP 2015, 769 Rn. 38; Seitz, Umschuldungsklauseln (Collective Action Clauses) in Staatsanleihen des europäischen Währungsraumes (2014), S. $269 \mathrm{ff}$.; Casper, BKR 2019, 209, $212 \mathrm{f}$.

${ }^{8}$ MüKo/Habersack (Fn. 1), Vor $\$ 793$ BGB Rn. 35; Seitz (Fn. 7), S. 269ff. - Allg. zum Begebungsvertrag s. noch unter IV.1. 
lisierte Sammelschuldbuchforderung nunmehr nach sachenrechtlichen Grundsätzen verfügt werden kann, ${ }^{9}$ was wiederum die Börsenfähigkeit der Anleihe gewährleistet.

Völlig anders die Lösung für Einzelschuldbuchforderungen: Für sie verzichtet das BSchuWG auf eine Fiktionslösung und bestimmt statt dessen in seinem $\$ 8$ Abs.1, dass Verfügungen zu ihrer Wirksamkeit gegenüber dem Schuldner neben der Zession der - konstitutiv wirkenden - Eintragung in das Bundesschuldbuch bedürfen; ungeachtet der fehlenden sachenrechtlichen Verankerung eröffnet $\$ 8$ Abs. 2, 3 BSchuWG sodann auf Grundlage der Eintragung die Möglichkeit des gutgläubigen Erwerbs der Forderung.

\section{Sammel-und Einzeleintragung nach dem eWpG}

Das BSchuWG dient dem als Art. 1 des Gesetzes zur Einführung von elektronischen Wertpapieren vom 3.6.2021 ${ }^{10}$ erlassenen eWpG als Vorbild: Es unterscheidet in $\$ 8 \mathrm{eWp}$ z zwischen der Sammeleintragung und der Einzeleintragung elektronischer Wertpapiere und qualifiziert in $\$ 9$ Abs. $1 \mathrm{eWpG}$ für elektronische Wertpapiere in Sammeleintragung die Berechtigen als Miteigentümer nach Bruchteilen an dem (als „Sammelbestand“ bezeichneten) elektronischen Wertpapier. Für einzeleingetragene elektronische Wertpapiere machen $\$ 24 \mathrm{ff}$. eWpG Verfügungen von der Eintragung oder Umtragung im elektronischen Wertpapierregister abhängig und statten das Register mit der Vermutung der Vollständigkeit und Richtigkeit aus, um so die Möglichkeit des gutgläubigen

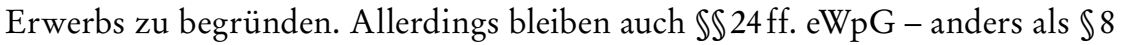
BSchuWG - insoweit sachenrechtlichen Denkmustern verhaftet, als auch für einzeleingetragene elektronische Wertpapiere die Fiktion des $\$ 2 \mathrm{Abs} .3 \mathrm{eWpG}$ gelten soll, wonach ein elektronisches Wertpapier als Sache im Sinne des $\$ 90$ BGB gilt, $\$ 25 \mathrm{eWpG}$ das Eigentum als Gegenstand der Übertragung ansieht und $\$ 27 \mathrm{eWp}$ an die Eintragung in das Register die Vermutung des Eigentums knüpft.

Überdies hat sich bereits der Regierungsentwurf in einem für die Systematik des geplanten Gesetzes durchaus entscheidenden Punkt von dem Referentenentwurf distanziert. ${ }^{11}$ Beide Entwürfe unterscheiden zwar zwischen dem zen-

\footnotetext{
9 So explizit Begr. RegE BWpVerwG, BT-Drs. 14/7010, S. 15.

10 BGBl. I 2021 S. 1423; zuvor RegE v. 16.12.2020, BT-Drs. 19/16925; Referentenentwurf vom 11.8.2020, abrufbar unter: https://www.bmjv.de/SharedDocs/Gesetzgebungsverfahren/ DE/Einfuehrung_elektr_Wertpapiere.html (alle Hyperlinks in diesem Beitrag wurden zuletzt am 20.05.2021 abgerufen).

${ }^{11}$ Für eine solche Änderung eine Reihe von Stellungnahmen zum Referentenentwurf, s. inbesondere Die Deutsche Kreditwirtschaft, Stellungnahme zum Referentenentwurf eWpG vom 14.9.2020, S.4f., abrufbar unter: https://www.bundesfinanzministerium.de/ Content/DE/Gesetzestexte/Gesetze_Gesetzesvorhaben/Abteilungen/Abteilung_VII/19_ Legislaturperiode/2020-08-11-einfuehrung-elektronische-wertpapiere/Stellungnahmedkw.pdf?__blob=publicationFile\&v=3.
} 
tralen Register, in das Zentralregisterwertpapiere einzutragen sind, und dem Kryptowertpapierregister, in dem Kryptowertpapiere zu erfassen sind. Auch unterscheiden beide Entwürfe in $\$ 3$ zwischen dem Inhaber, der als solcher in das Register eingetragen und damit formell legitimiert ist, und dem Berechtigten, der das Recht aus einem Wertpapier innehat und damit materiell berechtigt ist. Auf beide Unterscheidungen ist im weiteren Verlauf der Ausführungen zurückzukommen. Nach $\$ 8$ Abs. 1, 2 eWpG in der Fassung des Referentenentwurfs war allerdings das Kryptowertpapierregister sowohl für Einzeleintragungen als auch für Sammeleintragungen zugänglich, während Eintragungen in das zentrale Register nur in der Form der Sammeleintragung möglich sein sollten. Hingegen sehen bereits $\$ 8$ Abs. 1 eWpG in der Fassung des Regierungsentwurfs und sodann die Gesetz gewordene Fassung der Vorschrift vor, dass auch im zentralen Register Einzeleintragungen statthaft sind ${ }^{12}$ und Verfügungen über solchermaßen begründete Wertpapiere sodann den $\$ \int 24 \mathrm{ff}$. eWpG unterliegen. Hieran anknüpfend ermöglicht es $\$ 8$ Abs. $2 \mathrm{WpG}$, Einzeleintragungen auf Antrag des Inhabers in eine Sammeleintragung umzuwandeln ${ }^{13}$ und hierdurch die Börsenfähigkeit des elektronischen Wertpapiers herzustellen. ${ }^{14}$ Damit aber ist ein wesentlicher Unterschied zwischen zentralem Register und Kryptowertpapierregister entfallen: Wenn auch in der Praxis beim zentralen Register die Sammeleintragung und beim Kryptowertpapierregister die Einzeleintragung dominieren werden, so unterscheiden sich beide Register, was den Zugang zu ihnen betrifft, doch nur noch insoweit, als $\$ 16$ Abs. 1 eWpG besondere kryptografische Anforderungen an das Aufzeichnungssystem stellt. ${ }^{15}$

\section{Registerführung}

\section{Wertpapierregister im Allgemeinen}

\section{a) Pflichten und Haftung des Registerfübrers}

Die Registerführung liegt in der Verantwortung einer registerführenden Stelle. ${ }^{16}$ Während nach $\$ 12$ Abs. 2 eWpG die Führung des Zentralen Registers nur

12 S. ferner $\int \mathbb{S} 13$ Abs. 1 Nr. 5, 17 Abs. 1 Nr. 5 eWpG: das Register muss eine Kennzeichnung enthalten, ob es sich um eine Einzel- oder eine Sammeleintragung handelt; s. dazu noch unter IV.2.b).

13 Zur Überführung einer Sammeleintragung in eine Einzeleintragung s. \$9b Abs. 2 DepotG.

14 Begr. RegE eWpG (Fn.10), S.50; zur Börsenfähigkeit s. Art.3 Abs.1 VO (EU) Nr. 909/2014 vom 23.07.2014, ABl. EU Nr. L 257, S. 1 und dazu noch unter IV.2.c).

15 Dazu unter III.2.

${ }^{16} \mathrm{Zu}$ den Besonderheiten bei Kryptowertpapieren s. noch unter III.2.c), ferner Döding/ Wentz, Der Referentenentwurf zur Einführung von elektronischen Wertpapieren und Kryptowertpapieren, WM 2020, 2312, 2319; Lehmann, Zeitenwende im Wertpapierrecht, BKR 
durch eine Wertpapiersammelbank oder einen Verwahrer erfolgen kann, ${ }^{17} \mathrm{kann}$ der Emittent eines Kryptowertpapiers nach $\$ 16$ Abs. 2 S. 1 eWpG einen beliebigen Dienstleister, aber auch sich selbst benennen; nach $\$ 16$ Abs. 2 S. 2 eWpG gilt der Emittent als registerführende Stelle, wenn eine Benennung unterbleibt. Die Materialien zu $\$ 16$ Abs. 2 eWpG weisen zu Recht darauf hin, dass der Begriff der registerführenden Stelle formeller Natur ist (also eine Stelle als solche $\mathrm{zu}$ benennen ist), Verwaltung und Fortschreibung des Registers aber durchaus automatisiert und algorithmenbasiert erfolgen können. ${ }^{18}$

Die Pflichten der registerführenden Stelle sind ganz allgemein und unabhängig von der Art des elektronischen Wertpapiers und des Registers in $\$ 7$ Abs. 1 , 3 eWpG geregelt. Die Materialien machen es dem Registerführer zur Pflicht, in Bezug auf Datenspeicherung, Datendarstellung und Datensicherheit die jeweils effizientesten technischen Standards zu nutzen, ${ }^{19}$ und sprechen damit gewiss auch die Pflicht an, die verwendeten Systeme permament auf ihre Eignung und Verlässlichkeit zu überprüfen und gegebenenfalls fortzuentwickeln.

Missverständnisse können durch $\$ 7$ Abs. 2 S. 1 eWpG hervorgerufen werden, wonach die registerführende Stelle sicherzustellen hat, dass das elektronische Wertpapierregister „jederzeit die bestehende Rechtslage zutreffend wiedergibt und Eintragungen sowie Umtragungen vollständig und ordnungsgemäß erfolgen.“ Nachdem sich bei Sammeleintragung Verfügungen außerhalb des Registers vollziehen, ${ }^{20}$ kann es insoweit nur in Bezug auf die Person des Inhabers - der Wertpapiersammelbank oder des Verwahrers - zu einem Widerspruch zwischen bestehender Rechtslage und Registereintragung kommen. ${ }^{21}$

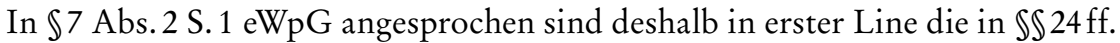
eWpG geregelten Verfügungen über einzeleingetragene elektronische Wertpapiere. ${ }^{22}$ Soweit die registerführende Stelle nach $\$ 7$ Abs. 2 S. 2 eWpG bei zu vertretender Pflichtverletzung „dem Berechtigten“ zum Ersatz des durch eine Satz 1 nicht entsprechende Registerführung entstehenden Schaden verpflichtet sein soll, muss dies selbstverständlich auch gegenüber einem vormals Berechtigten gelten, der durch fehlerhafte Registerführung seine Berechtigung verloren hat. ${ }^{23}$ Entsprechendes hat für den Haftungstatbestand des $\$ 7$ Abs. 3 S. 2 eWpG

2020, 431, 435; Sickinger/Thelen, Anleihen und Genussscheine auf der Blockchain, AG 2020, 862 Rn. 11.

17 Der Referentenentwurf (Fn. 10) sah noch eine Beschränkung auf Zentralverwahrer vor; zu Recht krit. Die Deutsche Kreditwirtschaft (Fn. 11), S.6f.

${ }_{18}$ Begr. RegE eWpG (Fn. 10), S. 60.

19 Begr. RegE eWpG (Fn. 10), S. 48 f.; krit. Dubovitskaya, Gesetzentwurf zur Einführung von elektronischen Wertpapieren: ein zaghafter Schritt nach vorn, ZIP 2020, 2551, $2560 \mathrm{f}$.

20 S. unter IV.2.c).

${ }^{21}$ Zutr. Segna, Elektronische Wertpapiere im zentralen Register, WM 2020, 2301, 2305.

22 Dazu unter IV.2.b).

${ }^{23}$ Dubovitskaya, ZIP 2020, 2551, 2561. 
zu gelten, auf den im Übrigen die in $\$ 7$ Abs. 2 S. 2 letzter Halbsatz eWpG geregelte Exkulpationsmöglichkeit analog zur Anwendung gelangen muss. ${ }^{24}$

\section{b) Registerberichtigung}

\$7 Abs.2 S. 2 eWpG gewährt dem durch fehlerhafte Registerführung um sein einzeleingetragenes Recht gebrachten Berechtigten zwar einen Schadensersatzanspruch. Einen dem Grundbuchberichtigungsanspruch vergleichbaren Registerberichtigungsanspruch, mit dem der Rechtsverlust verhindert werden kann, sieht der Entwurf hingegen nicht vor. ${ }^{25}$ Nach $\iint 14$ Abs. 1 S. 1 Nr. 2 lit.d), 18 Abs. 1 S. 1 Nr. 2 lit. d) eWpG darf die registerführende Stelle zwar Änderungen des Registerinhalts auch auf Grundlage einer gerichtlichen Entscheidung vornehmen; die Materialien verweisen insoweit insbesondere auf die in $\$ 894 \mathrm{ZPO}$ geregelte Fiktion der Abgabe einer Willenserklärung. ${ }^{26}$ Doch setzt dies einen materiell-rechtlichen Anspruch auf Abgabe der Willenserklärung voraus, an dem es vielfach fehlen wird. ${ }^{27}$

Dem Berechtigten bleibt deshalb allein ein Anspruch auf Herausgabe der Buchposition, den man vor dem Hintergrund der durch diese Position nach \$26 eWpG ermöglichten Verfügung gegenüber einem gutgläubigen Erwerber aus $₫ 812$ Abs. 1 S. 1 Alt. 1 oder $2 \mathrm{BGB}^{28}$ sowie gegebenenfalls aus der analogen Anwendung des $₫ 985 \mathrm{BGB}^{29}$ herleiten kann und der auf Umtragung gemäß $\ 4$ Abs. 8 eWpG gerichtet ist. ${ }^{30}$ Damit mag sich zwar ein Registerberichtigungsanspruch erübrigen, ${ }^{31}$ nicht aber die Möglichkeit der Eintragung eines den $\mathrm{He}$ rausgabeanspruch flankierenden und den Rechtsverlust infolge gutgläubigen Erwerbs ausschließenden Widerspruchs im Sinne von $\$ 899$ BGB, $\mathbb{1 6}$ Abs. 3 S. 3 GmbHG. ${ }^{32}$

24 Für Einführung eines entsprechenden Haftungsausschlusses bitkom, Stellungnahme zum Referentenentwurf eWpG vom 10.9.2020, S. 8 f., abrufbar unter: https://www. bundesfinanzministerium.de/Content/DE/Gesetzestexte/Gesetze_Gesetzesvorhaben/ Abteilungen/Abteilung_VII/19_Legislaturperiode/2020-08-11-einfuehrung-elektronischewertpapiere/Stellungnahme-bitkom.pdf?__blob=publicationFile\&v=3.

25 Dafür Möslein/Omlor/Urbach, Grundfragen eines Blockchain-Kapitalgesellschaftsrechts, ZIP 2020, 2149, 2160; Saive, Einführung elektronischer Wertpapiere, ZRP 2020, 219, 221; wohl auch Sickinger/Thelen, AG 2020, 862 Rn. $44 \mathrm{ff}$; dagegen Linardatos, Elektronische Schuldverschreibungen auf den Inhaber - des Wertpapiers neue Kleider, ZBB 2020, 329, 346.

26 Begr. RegE eWpG (Fn. 10), S. 58.

27 Sickinger/Thelen, AG 2020, 862 Rn. 45.

28 Dafür Sickinger/Thelen, AG 2020, 862 Rn. 46.

29 Dafür Linardatos, ZBB 2020, 329, 346.

30 Vgl. BGH, Urt. v. 30.11.2004, Az. XI ZR 200/03 = BGHZ 161, 189, $191 \mathrm{f}$.

31 Linardatos, ZBB 2020, 329, 346, der die ausdrückliche Regelung eines Herausgabeanspruchs (gerichtet auf Umtragung) empfiehlt.

32 Zutr. Sickinger/Thelen, AG 2020, 862 Rn. 46. 
c) Aufsicht

Die Beaufsichtigung der Registerführung obliegt nach $\$ 11 \mathrm{eWpG}$ der BaFin. $\$ 7$ Abs. 4 eWpG stellt zwar klar, dass die Registerführung als solche kein Depotgeschäft darstellt und deshalb keiner Erlaubnis nach $\$ 32 \mathrm{KWG}$ bedarf. Anderes gilt aber für die Übernahme der Verwahrfunktion gemäß $\$ 8$ Abs. 1 Nr. 1 eWpG. ${ }^{33}$ Unberührt bleiben im Übrigen der Tatbestand des Kryptoverwahrgeschäfts in $\$ 1$ Abs. 1a S. 2 Nr. 6 KWG ${ }^{34}$ der durch Art. 6 Nr. 2 lit. a) des Gesetzes zur Einführung von elektronischen Wertpapieren neu gefasst werden soll, sowie die Einführung des neuen Tatbestands der Kryptowertpapierregisterführung in $\$ 1$ Abs. 1a S. 2 Nr. 8 KWG; für beide Tatbestände sieht $\$ 2$ Abs. 7b KWG allerdings die Unanwendbarkeit zahlreicher aufsichtsrechtlicher Vorschriften vor. ${ }^{35}$

\section{Kryptowertpapierregister im Besonderen}

a) „Exklusivität"

Mögen auch die konzeptionellen Unterschiede zwischen dem Zentralen Register und dem Kryptowertpapierregister durch den Regierungsentwurf eingeebnet sein, ${ }^{36}$ so versteht es sich doch, dass zwischen beiden „Exklusivität“ in dem Sinne besteht, dass ein elektronisches Wertpapier immer nur in einem der beiden Register eingetragen sein kann. Der Emittent kann zwar nach $\$ 22$ eWpG ein bislang im Kryptowertpapierregister eingetragenes Wertpapier (und damit den Gesamtbestand) in ein anderes Kryptowertpapierregister oder in das zentrale Register übertragen, benötigt hierfür aber grundsätzlich die Zustimmung sämtlicher Wertpapierinhaber oder der Aufsichtsbehörde; in den Emissionsbedingungen kann bestimmt werden, dass die Zustimmung durch die Mehrheit der Inhaber genügt. ${ }^{37}$

\section{b) Sicherung der Funktionstüchtigkeit des Kryptowertpapierregisters}

Besondere Aufmerksamkeit widmet der Entwurf der Sicherung der Handelbarkeit des Kryptowertpapiers. Insoweit adressiert er zunächst den Emittenten. Er hat nach $\$ 21$ Abs. $1 \mathrm{eWpG}$ die erforderlichen technischen und organisatorischen Maßnahmen zu treffen, um die Integrität und Authentizität

\footnotetext{
${ }^{33}$ Begr. RegE eWpG (Fn. 10), S. 49.

${ }^{34}$ Dazu sowie zu weiteren aufsichtsrechtlich relevanten Tätigkeiten mit Kryptobezug Maume, in: ders./Maute (Hrsg.), Rechtshandbuch Kryptowerte (2020), $\$ 12 \mathrm{Rn} .42 \mathrm{ff}$.

35 Näher Begr. RegE eWpG (Fn. 10), S. 74; Döding/Wentz, WM 2020, 2312, 2319 f.; Dubovitskaya, ZIP 2020, 2551, 2558; Segna, WM 2020, 2301, 2303; für Ausnahme für Eigenemissionen Sickinger/Thelen, AG 2020, 862 Rn. 11 f.; krit. zum Lizenzerfordernis bitkom (Fn. 24), S. $1 \mathrm{f}$.

36 S. unter II.2.

37 Begr. RegE eWpG (Fn. 10), S. 65.
} 
der Kryptowertpapiere für die gesamte Dauer der Eintragung zu gewährleisten; die diesbezüglichen Anforderungen sollen nach $\$ 23$ Abs. 1 Nr. 25 eWpG durch Verordnung bestimmt werden. Hieran anknüpfend bestimmt $\$ 21$ Abs. 2 eWpG, dass bei Funktionsuntüchtigkeit des Kryptowertpapierregisters die Aufsichtsbehörde eine entsprechende Übertragung anordnen kann; die Materialien führen als Beispiel an, dass das Aufzeichnungssystem ungebräuchlich wird und sich für die zeitnahe Durchführung von Transaktionen nicht mehr genug „Miner“ finden. ${ }^{38}$ Die Übertragung steht denn auch nach $\$ 30$ S. $2 \mathrm{eWpG}$ der Wiederherstellung der Funktionstüchtigkeit des Registers gleich. ${ }^{39}$

Unterbleibt eine Übertragung, kann nach $\ 30$ S. 1 eWpG jeder Inhaber eines in einem Kryptowertpapierregister eingetragenen Wertpapiers dem Emittenten eine angemessene Frist zur Wiederherstellung der Funktionstüchtigkeit setzen und nach erfolglosem Ablauf der Frist die Schuldverschreibung außerordentlich kündigen. ${ }^{40}$ Das Gesetz spricht dieses Sonderkündigungsrecht explizit nur dem Inhaber einer in einem Kryptowertpapierregister eingetragenen „Schuldverschreibung “ zu, was vor dem Hintergrund des nach $\$ 1 \mathrm{eWp}$ auf Schuldverschreibungen begrenzten Anwendungsbereichs ohnehin selbstverständlich sein sollte; das Kündigungsrecht sollte deshalb, ohne dass damit eine sachliche Änderung verbunden wäre, dem Inhaber eines in einem Kryptowertpapierregister eingetragenen „elektronischen Wertpapiers“ zugestanden werden.

\section{c) Registerfübrung}

Eng mit dem vorstehend Gesagten zusammen hängen die in $\$ 16$ Abs. $1 \mathrm{eWpG}$ geregelten Anforderungen an das einem Kryptowertpapierregister zugrunde liegende Aufzeichnungssystem. Sie werden nach derzeitigem Stand der Technik in erster Linie, wenn nicht gar ausschließlich auf Basis der Distributed Ledger Technologie mit der ihr eigenen - in $\$ 4$ Abs. 11 eWpG explizit vorausgesetzten - dezentralen Speicherung und Verwaltung der Daten erfüllt. ${ }^{41}$ Diese Technologie bildet im Übrigen nicht nur die Grundlage des Registers, sondern typischerweise auch die des registrierten elektronischen Wertpapiers selbst. Bekannte Netzwerke wie namentlich Bitcoin oder Ethereum fallen freilich schon deshalb nicht unter $\int \mathbb{S} 16 \mathrm{ff}$. eWpG, weil die durch sie begründeten Positionen

\footnotetext{
38 Begr. RegE eWpG (Fn. 10), S. 65.

39 Die materielle Rechtslage bleibt unverändert, s. für die Übertragung nach $\$ 21 \mathrm{Abs.} 2$ eWpG Begr. RegE eWpG (Fn. 10), S. 65.

40 Allg. zur Frage eines Rechts zur außerordentlichen Kündigung der Schuldverschreibung BGH, Urt. v. 31.05.2016, Az. XI ZR 370/15 = BGHZ 210, 263 Rn.33; BGH, Urt. v. 14.02.2019, Az. IX ZR 149/16 = NZG 2019, 624 Rn.76; dazu sowie zu $\$ 313$ BGB s. MüKo/ Habersack (Fn. 1), \$793 BGB Rn.13 m.w. N.

${ }^{41}$ Begr. RegE eWpG (Fn. 10), S. 59; näher Döding/Wentz, WM 2020, 2312, 2318f.; speziell zum Erfordernis der „Fälschungssicherheit“ Linardatos, ZBB 2020, 329, 335; näher zur Distributed Ledger Technologie Scherer (Fn. 1), S. 92 ff.
} 
nicht Inhaberschuldverschreibung im Sinne des $\$ 1 \mathrm{eWpG}$ sind und es überdies an einem Emittenten im Sinne von $\$ 2$ Abs. 1 S. 2 eWpG fehlt. ${ }^{42}$

Auch für Schuldverschreibungen bleibt allerdings die Frage, wie sich das Erfordernis einer registerführenden Stelle ${ }^{43}$ mit der in $\$ \int 4$ Abs. 11, 16 Abs. 1 eWpG vorausgesetzten Fälschungssicherheit und Dezentralität des Aufzeichnungssystems in Einklang bringen lässt. Geht man davon aus, dass sich die Dezentralität nicht nur auf die Speicherung der Daten, sondern an sich auch auf die Verifizierung der Transaktion bezieht, ${ }^{44}$ so bliebe insbesondere fraglich, wie es zur Umsetzung einer Weisung im Sinne des $₫ 18$ Abs. 1 eWpG zur Änderung der Registerangaben durch die registerführende Stelle kommt ${ }^{45}$ und wie der Registerführer seiner Pflicht aus $\$ 18$ Abs. 5 eWpG nachkommen kann, eine Änderung im Kryptowertpapierregister rückgängig zu machen. ${ }^{46} \mathrm{Da}$ die Änderungen bei einem solchen Verständnis dezentral, nämlich durch die „Miner“ oder „Nodes“, vorgenommen werden, ${ }^{47}$ gibt es Stimmen, die es dann genügen lassen wollen, dass die registerführende Stelle die angewiesene Transaktion zum Vollzug durch die „Miner“ freigibt. ${ }^{48}$ Um diese Probleme zu vermeiden, erscheint es auch denkbar, die Blockchain nicht „permissionless“, sondern „permissioned“ auszugestalten und damit nur einzelne Teilnehmer - konkret: die registerführende Stelle - zur Verifizierung von Transaktionen zu berechtigen. ${ }^{49}$ In diesem Fall handelte es sich jedoch nicht um ein wirklich dezentrales Register, bei dem die einzelnen Transaktionen durch die verschiedenen „Nodes“ verifiziert werden; vielmehr erfolgte in diesem Fall nur die Speicherung der Daten dezentral.

Die Beachtung des in $\iint 14$ Abs. 3, 18 Abs. 3 eWpG geregelten Prioritätsprinzips dürfte hingegen im Kryptowertpapierregister schon deshalb unproblema-

${ }^{42}$ Näher zu Arten und Rechtsnatur blockchain-basierter Kryptotoken Maute, in: Maume/Maute (Hrsg.) (Fn. 34), \$4 Rn. 1 ff., 45 ff.; Scherer (Fn. 1), S. 108 ff.; Zickgraf, Initial Coin Offerings - Ein Fall für das Kapitalmarktrecht?, AG 2018, 293, $295 \mathrm{ff}$.

${ }^{43}$ Dazu unter III.1.a).

${ }^{44}$ Die Materialien sind diesbezüglich unklar, s. einerseits Begr. RegE eWpG (Fn.10), S.60, andererseits Begr. RegE eWpG (Fn.10), S. 44 („verteilten Infrastruktur mit entsprechend definierten Vorgaben zur dezentralen Kontrolle der Systeme").

${ }^{45}$ Nach $₫ 18$ Abs. 1 S. 5 eWpG darf die registerführende Stelle von einer Weisung des Inhabers gemäß $\$ 18$ Abs. 1 S. 1 Nr. 1 eWpG ausgehen, wenn die Weisung mittels eines geeigneten Authentifizierungsinstruments - und damit des Private Key des Inhabers (s. Lebmann, BKR 2020, 431, 435) - erteilt wurde.

46 S. Die Deutsche Kreditwirtschaft (Fn.11), S. 18; ferner Wieneke/Kunz, Das Gesetz zur Einführung von elektronischen Wertpapieren, NZG 2021, 316, 321. - Denkbar wäre, der registerführenden Stelle eine Art „Master-Private-Key“ einzuräumen.

47 Fromberger/Zimmermann, in: Maume/Maute (Hrsg.) (Fn. 34), $\$ 1$ Rn. $15 \mathrm{ff}$.

48 So bitkom (Fn. 24), S. 7; zustimmend auch Dubovitskaya, ZIP 2020, 2551, 2560.

49 Begr. RegE eWpG (Fn. 10), S.60; Döding/Wentz, WM 2020, 2312, 2319; allg. zu der im Text genannten Unterscheidung Fromberger/Zimmermann, in: Maume/Maute (Hrsg.) (Fn. 34), \$1 Rn.9aff. 
tisch sein, weil die Daten, wie in $\$ 16$ Abs. 1 eWpG explizit vorgeschrieben, in der Zeitfolge protokolliert werden. ${ }^{50}$

\section{Materielles Registerrecht}

\section{Begebung des elektronischen Wertpapiers}

\section{a) Eintragung und Begebungsvertrag}

Dem Register kommt zunächst bei Begebung des elektronischen Wertpapiers eine entscheidende Funktion zu. Nach $\$ 2$ Abs. 1 S. 2 eWpG wird nämlich ein elektronisches Wertpapier - mag es Zentralregisterwertpapier oder Kryptowertpapier sein - dadurch begeben, dass der Emittent an Stelle der Ausstellung einer Wertpapierurkunde eine Eintragung in ein elektronisches Wertpapierregister im Sinne des $₫ 4$ Abs. 1 eWpG und damit in ein zentrales Register gemäß \12 oder ein Kryptowertpapierregister gemäß $\$ 16$ bewirkt. Vor dem Hintergrund der Sachfiktion des $\$ 2$ Abs. 3 eWpG, die für die sachenrechtliche Verankerkung aller elektronischen Wertpapiere sorgen soll, ist es nur konsequent, dass $\$ 2$ Abs. 1 S. 2 eWpG die Eintragung als Substitut für die in $\$ 793$ Abs. 1 S. 1 BGB vorausgesetzte Ausstellung einer Urkunde ansieht und die Materialien in dieser - in $\$ 4$ Abs. 4 eWpG näher definierten - Eintragung nicht mehr als einen „Skripturakt“ erblicken, so dass die sonstigen Voraussetzungen der Entstehung des Wertpapiers und des im Wertpapier verkörperten Rechts und damit der Abschluss eines Begebungsvertrags unberührt bleiben. ${ }^{51}$ Bedeutsam ist dies, worauf die Begründung zu Recht hinweist, vor allem für eine bereits bestehende Forderung auf Basis der Distributed Ledger Technologie: Allein durch die nachfolgende Registrierung erlangt sie noch nicht den Charakter eines Kryptowertpapiers. $^{52}$

\section{b) Niederlegung der Emissionsbedingungen}

Von der Eintragung zu unterscheiden ist die Niederlegung der Emissionsbedingungen. Sie hat nach $\ 5$ Abs. 1 S. $1 \mathrm{eWpG}$ vor der Eintragung zu erfolgen, und zwar in der Form, dass die Bedingungen bei der registerführenden Stelle

50 Linardatos, ZBB 2020, 329, $341 \mathrm{f}$., dort auch zum Sonderfall konkurrierender Sicherungsrechte.

51 Begr. RegE eWpG (Fn. 10), S. 39, 42; näher zu den Voraussetzungen wertpapiermäßiger Verpflichtung MüKo/Habersack (Fn. 1), Vor $\$ 793$ BGB Rn. 24ff. m. w. N.; zur entsprechenden Diskussion in Österreich s. Ebner/Kalss, Die digitale Sammelurkunde - ein erster Schritt zur vollständigen Digitalisierung des österreichischen Wertpapierrechts, GesRZ 2020, 369, 378.

52 Begr. RegE eWpG (Fn. 10), S. 42. 
als beständiges elektronisches Dokument jedermann ${ }^{53}$ zur beliebig wiederholbaren unmittelbaren Kenntnisnahme zugänglich zu machen sind. ${ }^{54} \mathrm{Un}$ terbleibt eine ordnungsgemäße Niederlegung, wird man allerdings entgegen einer im Schrifttum geäußerten Ansicht die wirksame Erstbegebung des Wertpapiers nicht in Frage stellen können. ${ }^{55}$ Hierfür spricht auch ein Blick auf den elektronischen Anteilsschein, für den $\mathbb{\$} 95$ Abs. 3 KAGB erst gar nicht auf $\$ 5$ eWpG verweist und deshalb auf eine Niederlegung der Anlagebedingungen verzichtet. Wertpapier- und AGB-rechtlich betrachtet stellt sich die Niederlegung der Emissionsbedingungen vielmehr als besondere Form der Einbeziehung der Nebenbedingungen dar. ${ }^{56}$ Scheitert diese, bleibt das Hauptleistungsversprechen des Emittenten unberührt, soweit sich dieses den nach $\mathbb{S} \$ 13 \mathrm{Abs.} 1$ Nr. 1, 17 Abs. 1 Nr. 1 eWpG geschuldeten Registerangaben entnehmen lässt.

\section{Formelle Legitimation und materielle Berechtigung \\ a) Grundlagen}

Die Registereintragung soll, vergleichbar mit der Ausstellung einer Urkunde, für die Publizität des im Wertpapier verkörperten Versprechens sorgen und im Idealfall damit eine Grundlage für die Umlauffähigkeit des Wertpapiers und die Möglichkeit des Erwerbs vom Nichtberechtigten gewährleisten. Letzteres bildet gleichsam die Daseinsberechtigung der sachenrechtlichen Fundierung des deutschen Wertpapierrechts und erklärt zugleich die - auch in $\$ 3$ eWpG verlautbarte - Unterscheidung zwischen dem formell legitimierten Inhaber eines Wertpapiers und dem materiell Berechtigten, im Falle einer Urkunde also demjenigen, der Eigentümer derselben ist und damit auch das Recht aus dem Papier innehat.

Bei einem herkömmlichen Inhaberpapier verhält es sich bekanntlich so, dass die Inhaberschaft an den Besitz an der Urkunde anknüpft und - im Sinne einer widerlegbaren Vermutung - sowohl den Inhaber als Berechtigten legitimiert als auch dem Schuldner die befreiende Leistung an den Inhaber ermöglicht. ${ }^{57}$ Bei einem elektronischen Wertpapier wird nach $\$ 3$ Abs. $1 \mathrm{eWpG}$ die Person des Inhabers durch die Eintragung bestimmt, und zwar, wie die Materialien zu Recht betonen, , unabhängig davon, ob diese Person von der Eintragung weiß, berechtigt ist oder tatsächlich über das elektronische Wertpapier verfügen kann. “" 58 Nicht nur hierdurch unterscheidet sich der Inhaberbegriff des $\$ 3$ Abs. 1 eWpG

${ }^{53}$ Krit. hierzu Die Deutsche Kreditwirtschaft (Fn.11), S. 9; Wieneke/Kunz, NZG 2021, 316, 319 .

54 Näher Döding/Wentz, WM 2020, 2312, 2315.

55 So aber Linardatos, ZBB 2020, 329, 339.

56 Näher zur Frage einer Einbeziehungs- und Inhaltskontrolle von Emissionsbedingungen MüKo/Habersack (Fn. 1), \$793 BGB Rn. $50 \mathrm{ff}$.

57 MüKo/Habersack (Fn. 1), Vor $\$ 793$ BGB Rn. 16, $\$ 793$ BGB Rn. 29 ff. (39), 41 f. m. w. N.

58 Begr. RegE eWpG (Fn. 10), S. 4. 
von demjenigen des herkömmlichen Wertpapierrechts. Im Einzelnen ist zwischen der Einzel- und der Sammeleintragung zu unterscheiden:

\section{b) Einzeleintragung}

Für die - nach der Konzeption des eWpG ${ }^{59}$ sowohl bei Zentralregisterwertpapieren als auch bei Kryptowertpapieren mögliche - Einzeleintragung strebt das Gesetz einen weitgehenden Gleichlauf von formeller Legitimation und materieller Berechtigung an. Dieser soll vor allem durch $\$ 24 \mathrm{eWpG}$ hergestellt werden, der die Wirksamkeit einer jeden Verfügung über das elektronische Wertpapier und über ein Recht aus einem elektronischen Wertpapier ${ }^{60}$ an die Verlautbarung der Rechtsänderung durch das Register abhängig macht; hierdurch wird auch über die Begebung des Wertpapiers hinaus der sodann in $\$ 28$ Abs. 1 eWpG - allerdings ganz allgemein und damit auch für die Sammeleintragung $^{61}$ - ausdrücklich geregelten Legitimations- und Liberationswirkung der Eintragung eine hinreichende Grundlage verliehen.

Etwaigen Diskrepanzen zwischen Inhaberschaft und Berechtigung, wie sie beispielsweise bei Unwirksamkeit einer gleichwohl eingetragenen Verfügung über das elektronische Wertpapier begegnen können, trägt $\$ 26 \mathrm{eWpG}$ Rechnung, der - allerdings nur für einzeleingetragene Wertpapiere - die Vermutung der Vollständigkeit und Richtigkeit des Registers statuiert und hierdurch den Erwerb vom Nichtberechtigten ermöglicht. Vor diesem Hintergrund sind $\$ \int 13$ Abs. 2 S. 1, Abs. 3, 17 Abs. 2 S. 1, Abs. 3 eWpG zu sehen, wonach der Registerführer bei Einzeltragung sicherzustellen hat, dass das Register Angaben über Verfügungshindernisse und Rechte Dritter enthält und diese Angaben mit den Angaben nach $\$ \int 13$ Abs.1, 17 Abs. 1 eWpG so verknüpft werden, dass sie nur zusammen abgerufen werden können und hierdurch Bösgläubigkeit des Erwerbers sichergestellt ist. Zu den Verfügungshindernissen zählt beispielsweise der Verlust der Verfügungsbefugnis infolge Insolvenzeröffnung ( $\$ 80 \mathrm{InsO}$ ), aber auch das Fehlen oder die Beschränkung der Geschäftsfähigkeit insoweit hat allerdings $₫ 26$ S. 3 eWpG die noch im Regierungsentwurf vorgesehene Möglichkeit des gutgläubigen Erwerbs beseitigt. ${ }^{62}$ Eintragungen dieser Art sind deklaratorischer Natur. Rechte Dritter - etwa ein Pfandrecht - hingegen entstehen, soweit sie durch Rechtsgeschäft begründet werden, nach $\$ 24 \mathrm{Nr} .1 \mathrm{eWpG}$ erst durch konstitutiv wirkende Eintragung; ein gutgläubiger lastenfreier Erwerb gemäß $\ 26 \mathrm{eWpG}$ wird durch die Verlautbarung des beschränkten dinglichen Rechts durch das Register verhindert.

59 Dazu sowie zum abweichenden Ansatz des Referentenentwurfs unter II.2.

60 Näher dazu sowie zu $\$ 24$ Nr.3 eWpG Bialluch-v. Allwörden, Zivil- und prospektrechtliche Aspekte des eWpG, RDi 2021, 13 Rn. $11 \mathrm{ff}$.

61 Dazu noch unter IV.2.c).

62 Lebmann, NJW 2021, 2318, 2322; zur abw. Entwurfsfassung S. 13. Begr. RegE eWpG (Fn. 10), S. 56 f. 
Können somit $\$ \mathbb{2 4 , 2 6}$ eWpG zumindest ihrem gedanklichen Ansatz nach durchaus auch über die Erstbegebung des elektronischen Wertpapiers hinaus für einen dem herkömmlichen Wertpapierrecht sogar überlegenen Gleichlauf von formeller Inhaberschaft und materieller Berechtigung sorgen, ${ }^{63}$ so liegen die diesbezüglichen Probleme der einzeleingetragenen elektronischen Wertpapiere auf einer anderen Ebene. Für den vom Gesetzgeber im Zusammenhang mit einzeleintragenen Wertpapieren vermutlich in erster Linie ins Auge gefassten Fall des Kryptowertpapiers ist nämlich schon die Frage, wie sich die für eine Verfügung unabdingbare Einigung und die in $\$ 25 \mathrm{Abs.} 1 \mathrm{eWpG}$ speziell für die Übereignung zusätzlich vorausgesetzte Weisung des Berechtigten in der Kryptowelt vollziehen sollen, nachdem der veräußerungswillige BlockchainTeilnehmer unter Rückgriff auf Public Key und Private Key eine entsprechende Transaktionsnachricht an die Blockchain übermittelt und diese Nachricht nicht gezielt an den Erwerber gesendet wird; dieser Frage braucht in einem Registerfragen gewidmeten Beitrag nicht näher nachgegangen zu werden. ${ }^{64}$

Aber auch die in $\$ 25$ Abs. $1 \mathrm{eWpG}$ für die Übereignung des einzeleingetragenen elektronischen Wertpapiers vorausgesetzte „Umtragung“ - und damit die Ersetzung des bislang eingetragenen Veräußerers durch den Erwerber $(\mathbb{S} 4$ Abs. 8 eWpG) - dürfte sich nach den derzeitigen Gegebenheiten der BlockchainTechnologie jedenfalls nicht im Wege einer separaten Ein- und Austragung vollziehen lassen..$^{65} \mathrm{Ob}$ die Voraussetzungen einer Umtragung dadurch erfüllt werden können, dass die Veräußerung auf der Block vermerkt wird, erscheint alles andere als gewiss. Die Definition der Umtragung in $\$ 4$ Abs. 8 eWpG („Ersetzung des Inhabers [...] durch einen neuen Inhaber") ist jedenfalls vor dem Hintergrund der Blockchain-Technologie unsauber, wenn man mit der Amtlichen Begründung darin eine Austragung des bisherigen Inhabers und eine Eintragung des neuen Inhabers versteht. ${ }^{66} \mathrm{Da}$ bei der Blockchain keine Austragung des bisherigen Inhabers stattfindet, vielmehr die personelle Zuordnung des jeweiligen Verfügungsobjekts fortlaufend fortgeschrieben wird, ergibt sich

${ }^{63}$ So allgemein Begr. RegE eWpG (Fn. 10), S. 55.

64 Näher Dubovitskaya, ZIP 2020, 2551, 2559; Lehmann, BKR 2020, 431, 436f.; Linardatos, ZBB 2020, 329, 340; Sickinger/Thelen, AG 2020, 862 Rn. 24f.; Wieneke/Kunz, NZG 2021, 316, 322; für die Übertragung von Token Zickgraf, in: Maume/Maute (Hrsg.) (Fn. 34), $\$ 11$ Rn. 35 m. w. N.; allgemein zum Ablauf von blockchain-basierten Transaktionen Fromberger/ Zimmermann, in: Maume/Maute (Hrsg.) (Fn. 34), \$1 Rn. $1 \mathrm{ff} ., 15 \mathrm{ff}$.

65 Lehmann, BKR 2020, 431, 436; Linardatos, ZBB 2020, 329, 341; s. ferner Mittwoch, Der Entwurf eines Gesetzes zur Einführung elektronischer Wertpapiere - ein Quantensprung für das Zivil- und Finanzmarktrecht?, WM 2021, 375, 381; Fromberger/Zimmermann, in: Maume/Maute (Hrsg.) (Fn. 34), $\$ 1$ Rn. 18 f.

${ }^{66}$ Begr. RegE eWpG (Fn. 10), S. 43: „Der Erwerber wird Inhaber in dem Augenblick, in dem er als solcher in das Wertpapierregister anstelle des bisherigen Inhabers eingetragen wird. [...] Zugleich wird für den Fall der Übereignung von Wertpapieren in Einzeleintragung mit $\$ 25$ Absatz 1 Satz 2 klargestellt, dass der Veräußerer nach Austragung keinen (Eigentums-) Rechtsverlust erleidet, solange die Eintragung noch nicht erfolgt ist.“ 
die aktuelle Inhaberschaft aus der Kette der vorherigen - und konkret dem jeweils letzten der - Übertragungsvorgänge bezüglich des jeweiligen Verfügungsobjekts (und damit der konkreten Schuldverschreibung). Als Umtragung kann daher - zumindest für DLT-Systeme wie die Blockchain - nur die Fortschreibung der Kette und damit die Eintragung der Transaktion angesehen werden. ${ }^{67}$ Dies ist vermutlich auch die Vorstellung der Entwurfsverfasser, zumal $\$ 3$ Abs. 1 eWpG als „Inhaber“ denjenigen bezeichnet, der „als Inhaber [...] im elektronischen Wertpapierregister eingetragen ist"; eine entsprechende Klarstellung in $\$ 4 \mathrm{Abs.} 8 \mathrm{eWpG}$ dürfte sich dennoch empfehlen. Davon unberührt bleibt im Übrigen die Möglichkeit, aus dem chronologischen Verzeichnis aller Transaktionen in der Blockchain ein „virtuelles“ Register herzuleiten, um hierdurch auch für Kryptowertpapiere eine wirkliche Registerpublizität sicherzustellen. Insgesamt stellt sich aber die Frage, ob einer solchen Registerpublizität in der Praxis irgendein Nutzen zukäme. ${ }^{68}$

Wie dem auch sei: Indem $\mathbb{\$} 24 \mathrm{ff}$. eWpG für Verfügungen über einzeleingetragene elektronische Wertpapiere an konstitutiv wirkende Eintragungen im Register anknüpfen, übertragen sie die Welt der Urkunde zwar durchaus systemgerecht auf die digitale Welt. Doch dürfte sich das Modell am ehesten für einzeleingetragene Zentralregisterwertpapiere eignen; deren praktische Bedeutung dürfte schon angesichts der ihnen fehlenden Börsenfähigkeit überschaubar bleiben. Bei Kryptowertpapieren liegen die Schwierigkeiten darin begründet, dass sich die Anforderungen an den Verfügungstatbestand nicht ohne Weiteres mit den Gegebenheiten der Blockchain-Technologie in Einklang bringen lassen. Damit aber ist nicht auszuschließen, dass die Vorschriften über einzeleingetragene elektronische Wertpapiere weitgehen leerlaufen und das eWpG auch insoweit ${ }^{69}$ die Erwartungen der Entwurfsverfasser und der Praxis enttäuschen wird.

\section{c) Sammeleintragung}

Gänzlich anders ist die Ausgangslage bei der Sammeleintragung von Zentralregister- oder Kryptowertpapieren. In diesem Fall muss eine durchgehende und damit jeden Folgeerwerb umfassende Verfügungstransparenz, wie sie für einzeleingetragene Wertpapiere in $\$ 24 \mathrm{eWp}$ vorgesehen ist, schon an ihrer

${ }^{67}$ Instruktiv Fromberger/Zimmermann, in: Maume/Maute (Hrsg.) (Fn. 34), $\$ 1$ Rn. $19 \mathrm{ff}$.

68 Lehmann, BKR 2020, 431, 437 weist zu Recht darauf hin auf, dass die Erwartung einer Einsichtnahme durch den Erwerber vor der jeweiligen Transaktion „lebensfremd“ sei und nicht auf den schnellen Verkehr mit Inhaberschuldverschreibungen passe; überdies weist Lebmann (a.a. O.) zu Recht darauf hin, dass bei der Blockchain die (Verfügungs-)Berechtigung mithilfe kryptographischer Verfahren durch die Signierung mit dem private key nachgewiesen werde und es gerade keiner Einsichtnahme in ein Register - und damit auch keiner Registerpublizität - bedürfe.

${ }_{69} \mathrm{Zu}$ den Problemen des Registermodells im Rahmen blockchain-basierter elektronischer Wertpapiere s. unter III.2.; zu den mit der Übertragung des sammeleingetragenen Wertpapiers verbundenen Schwierigkeiten s. unter IV.2.c). 
Unvereinbarkeit mit Art. 3 Abs. 1 CSDR ${ }^{70}$ scheitern; bei Erfordernis einer konstitutiv wirkenden Ein- oder Umtragung wäre schlicht nicht gewährleistet, „dass diese Wertpapiere durch Buchungen im Effektengiro erfasst werden, in dem die Wertpapiere entweder immobilisiert werden oder von Anfang an in entmaterialisierter Form begeben werden." Vor diesem Hintergrund folgt $\$ 8$ Abs. 1 Nr. $1 \mathrm{eWp}$ dem Modell des $\$ 6$ Abs. $1 \mathrm{BSchuWG}^{71}$ und bestimmt, dass als Inhaber des im Wege der Sammeleintragung einzutragenden elektronischen Wertpapiers eine Wertpapiersammelbank oder ein Verwahrer einzutragen ist. Praktisch bedeutsam dürfte allein die Eintragung einer Wertpapiersammelbank sein: Bei ihr handelt es sich nach $\$ 4$ Abs. $5 \mathrm{eWpG}$ um eine nach Art. $16 \mathrm{CSDR}^{72}$ als Zentralverwahrer zugelassene juristische Person, hierzulande also die Clearstream Banking AG; die Sammeleintragung eines (anderen) Verwahrers kommt nur in Betracht, wenn das Wertpapier nicht an einer Börse oder einem anderen Handelsplatz im Sinne der CSDR gehandelt werden soll.

Nach $\$ 9$ Abs. 2 S. 1 eWpG verwaltet die Wertpapiersammelbank oder der Verwahrer die Sammeleintragung treuhänderisch für die Berechtigten, ohne selbst Berechtigter zu sein. Die Wertpapiersammelbank oder der Verwahrer ist mit anderen Worten nicht Vollrechtsinhaber, sondern im Sinne des $\$ 185$ Abs. 1 BGB ermächtigter Treuhänder, ${ }^{73}$ mithin nur „Inhaber“ im Sinne des $\$ 3$ Abs. 1 eWpG und damit Buchberechtigter; hingegen gelten nach $\$ 9$ Abs. 1 S. 1 eWpG das elektronische Wertpapier in Sammeleintragung als Wertpapiersammelbestand und die Berechtigten der eingetragenen inhaltsgleichen Rechte nach $\$ 9$ Abs. 1 S. 2 eWpG als Miteigentümer nach Bruchteilen an dem eingetragenen elektronischen Wertpapier.

Da die Eintragung nach $\int \$ 13$ Abs. 1 Nr. 5, 17 Abs. 1 Nr. 5 eWpG als Sammeleintragung gekennzeichnet ist, halten die Materialien einen Vermerk, dass die Wertpapiersammelbank oder der Verwahrer nicht materiell Berechtigter, sondern nur ermächtigter Treuhänder ist, für entbehrlich. ${ }^{74}$ Auch wenn deshalb anzunehmen ist, dass das Register, soweit es die Wertpapiersammelbank oder den Verwahrer als Inhaber und den Umstand der Sammeleintragung ausweist, die tatsächliche Rechtslage mit Blick auf die als bekannt vorauszusetzenden Vorschriften des $₫ 9$ Abs. 1, 2 eWpG durchaus zutreffend ausweist, ${ }^{75}$ wird deutlich, dass das Register im Falle der Sammeleintragung nur der erstmaligen Begebung des elektronischen Wertpapiers dient und gerade nicht die Funktion hat, die materiell Berechtigten auszuweisen oder gar die Wirksamkeit einer Rechtsänderung an eine entsprechende Verlautbarung durch das Register zu binden. Sieht

\footnotetext{
70 VO (EU) Nr. 909/2014 vom 23.07.2014, ABl. EU Nr. L 257, S. 1; s. bereits unter II.2.

71 S. unter I.2.a).

72 VO (EU) Nr. 909/2014 vom 23.07.2014, ABl. EU Nr. L 257, S. 1; s. bereits unter II.2.

${ }^{73}$ Dubovitskaya, ZIP 2020, 2551, 2556; Segna, WM 2020, 2301, 2308.

74 Begr. RegE eWpG (Fn. 10), S. 50.

75 Von „permanenter Unrichtigkeit“ spricht hingegen Segna, WM 2020, 2301, 2310.
} 
man von eigenen Beständen der eingetragenen Wertpapiersammelbank oder des eingetragenen Verwahrers ab, geht die materielle Rechtslage mit anderen Worten zu keinem Zeitpunkt aus dem Register hervor; sie ergibt sich vielmehr aus den Depotkonten der Anleger. ${ }^{76}$

Vergleicht man die Rechtslage nach $\iint 8 \mathrm{f}$. eWpG mit der bisherigen Rechtslage, so muss deshalb konstatiert werden, dass bei Lichte betrachtet nur insoweit ein Unterschied besteht, als nach dem eWpG die Einlieferung einer Globalurkunde durch die Registrierung eines elektronischen Wertpapiers ersetzt werden kann. ${ }^{77}$ Die Materialien sehen denn auch in der Eintragung der Wertpapiersammelbank (der Sache nach: der Clearstream Banking AG) als Wertpapierinhaber und der Registerführung durch die Wertpapiersammelbank die „digitale Abbildung des institutionellen Effektengiroverkehrs“ und erblicken in der Entbehrlichkeit der Verwahrung einer Papierurkunde den wesentlichen Beitrag zur Senkung der Transaktionskosten $;^{78}$ nach $\$ 12$ Abs. 3 eWpG wird ein auf die Wertpapiersammelbank lautendes und in ein von der Wertpapiersammelbank geführtes Zentralregister eingetragenes Wertpapier „zur Abwicklung im Effektengiro bei einer Wertpapiersammelbank erfasst."

Der in dem Verzicht auf eine Urkunde liegende Vorteil ist allerdings nicht nur eher unbedeutend, sondern wird zudem durch eine Reihe von Ungewissheiten und Unwägbarkeiten erkauft. Die Sachfiktion des $\$ 2$ Abs. 3 eWpG und die hieran anknüpfende Fiktion des Miteigentums des Berechtigten in $\ 9$ Abs. 1 S. 2 eWpG sichern dem Berechtigten zwar in der Insolvenz der Wertpapiersammelbank oder eines Intermediärs die Rechte aus \47 InsO, \771 ZPO. ${ }^{80}$ Nicht wirklich klar ist freilich, wie sich in Ermangelung einer papierenen Urkunde und auf Grundlage der Fiktionen der $\$ \$ \$ 2$ Abs. 3, 9 Abs. 1 S. 2 eWpG eine Übertragung des Miteigentums (die anders als bei Einzeleintragung außerhalb des Registers erfolgt) konkret vollziehen soll. Ausweislich der Materialien soll die Wertpapiersammelbank oder der Verwahrer für die Miteigentümer „den unmittelbaren Besitz an der fingierten Sache ,Sammeleintragung““ halt-

76 Segna, WM 2020, 2301, 2305; Sickinger/Thelen, AG 2020, 862 Rn. 19; Wieneke/Kunz, NZG 2021, 316, 319.

77 Zutr. Lehmann, BKR 2020, 431, 438; Sickinger/Thelen, AG 2020, 862 Rn. 10; Wieneke/Kunz, NZG 2021, 316, 319; wohlwollende Beurteilung dieses Umstands bei Mittwoch, WM 2021, 375, $381 \mathrm{f}$.

78 Begr. RegE eWpG (Fn. 10), S. 49.

79 Dazu Lehmann, BKR 2020, 431, 435; s. auch lindenpartners, Stellungnahme zum Referentenentwurf eWpG vom 14.9.2020, S.6f., die sich für die Möglichkeit der Einbuchung von sammeleingetragenen Kryptowertpapieren im Effektengiro aussprechen, abrufbar unter: https://www.bundesfinanzministerium.de/Content/DE/Gesetzestexte/Gesetze_Ge setzesvorhaben/Abteilungen/Abteilung_VII/19_Legislaturperiode/2020-08-11-einfueh rung-elektronische-wertpapiere/Stellungnahme-lindenpartners.pdf?_blob=publication File\&v=2.

80 Segna, WM 2020, 2301, 2307. 
en, ${ }^{81}$ was darauf schließen lässt, dass mit der Sach- und Miteigentumsfiktion eine Besitzfiktion verbunden sein soll und Verfügungen über das Miteigentum deshalb ungeachtet des Fehlens einer Urkunde nach den etablierten Grundsätzen über die Verfügung über das Miteigentum an der Globalurkunde ${ }^{82}$ vonstatten gehen können. ${ }^{83}$

Nachdem sich diese Grundsätze freilich schon für die papierene Globalurkunde - durchaus zu Recht ${ }^{84}$ - dem Vorwurf ausgesetzt sehen, Mitbesitz des Veräußerers zu fingieren, muss dies für eine Anknüpfung an die Buchposition „den unmittelbaren Besitz an der fingierten Sache, Sammeleintragung “" 85 - erst recht gelten. Hinzu kommt das Problem, dass dem Emittenten das Auseinanderfallen von Inhaberschaft und Berechtigung bekannt ist und deshalb unklar ist, wie im Falle der Sammeleintragung mit der in $\$ 28$ Abs. 1 eWpG auch insoweit vorgesehenen Legitimations- und Liberationswirkung umzugehen ist. ${ }^{86}$ Zwar soll dem Berechtigten in $\$ 6$ Abs. 2 DepotG ein Anspruch auf Erteilung einer Depotbescheinigung zugebilligt werden und der Hinterleger hierdurch zur Rechtsausübung legitimiert werden; das Verhältnis zu $\$ 28$ Abs. 1 eWpG bleibt gleichwohl unklar.

Insgesamt ist die Lösung des Regierungsentwurfs deshalb für den für den Kapitalmarkt bedeutsamen Fall der Sammeleintragung recht unvollkommen. Der Entwurf verzichtet auf jegliche Verfügungstransparenz und belastet den Verfügungstatbestand mit Unwägbarkeiten und Unzulänglichkeiten, die über diejenigen, die das geltende System der papierenen Globalurkunde birgt, noch hinausgehen. Auf der Haben-Seite steht allein der überaus bescheidene Vorteil, dass künftig die Einlieferung einer Globalurkunde durch die Registrierung eines elektronischen Wertpapiers ersetzt werden kann.

\section{Fazit}

Das eWpG ist, dies sollten die vorstehenden Überlegungen verdeutlicht haben, nicht der „große Wurf“, den das deutsche Wertpapierrecht zur Beseitigung des einleitend erwähnten Dilemmas benötigt. ${ }^{87}$ Dem Gesetzgeber ist dies zwar durchaus bewusst, sieht er doch in der Möglichkeit, Schuldverschreibungen 436.

${ }^{81}$ Begr. RegE eWpG (Fn. 10), S. 49; berechtigt die Kritik von Lehmann, BKR 2020, 431,

82 Dazu unter I.1.

83 So auch Lehmann, BKR 2020, 431, 436; s. ferner Bialluch-v. Allwörden, RDi 2021, 13

Rn.16f.; zweifelnd Segna, WM 2020, 2301, 2309.

${ }^{84}$ S. die Nachw. in Fn. 4.

${ }^{85}$ Begr. RegE eWpG (Fn. 10), S. 49; berechtigte Kritik bei Lebmann, BKR 2020, 433, 436.

${ }^{86}$ Näher Dubovitskaya, ZIP 2020, 2551, $2556 f$.

87 Kritische Bewertung auch bei Bialluch-v. Allwörden, RDi 2021, 13 Rn.30; Casper, BKR 2019, 209, 217; Dubovitskaya, ZIP 2020, 2551, 2561; Labusen, Das Sachenrecht der elek- 
elektronisch zu begeben, nur einen „ersten Schritt“ auf dem Weg zu einer generellen Öffnung des deutschen Rechts für elektronische Wertpapiere. ${ }^{88}$ Vorzuwerfen ist dem Gesetz indes nicht die Beschränkung seines sachlichen Anwendungsbereichs, sondern sein Festhalten an der überkommenen Dogmatik und sachenrechtlichen Verankerung des Wertpapierrechts.

Dass diese überkommene Dogmatik zur Bewältigung der mit dem modernen Effektengiroverkehr verbundenen Rechtsfragen - noch dazu im digitalen Zeitalter - überfordert ist, zeigt sich vor allem im Zusammenhang mit der für den Handel am Kapitalmarkt unerlässlichen Sammeleintragung. Insoweit strebt das Gesetz erst gar nicht an, dass das Register die materielle Rechtslage abbildet. Erschwerend hinzu kommt, dass das sachenrechtliche Denken den Verfügungstatbestand mit Unwägbarkeiten und Unzulänglichkeiten belastet, die über diejenigen, die das geltende System der papierenen Globalurkunde birgt, noch hinausgehen. Dass nun die Einlieferung einer Globalurkunde durch die Registrierung eines elektronischen Wertpapiers unter Eintragung einer Wertpapiersammelbank oder eines Verwahres als Wertpapierinhaber ersetzt werden kann, ist angesichts der Zementierung und Vertiefung der mit der sachenrechtlichen Anknüpfung verbundenen Probleme ein zu schwacher Trost.

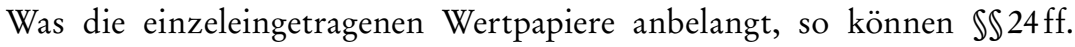
eWpG zwar die von ihnen angestrebte vollständige Verfügungstransparenz gewährleisten. Die Probleme liegen insoweit indes darin begründet, dass sich die den Beteiligten im Zusammenhang mit Verfügungen und Registerführung abverlangten Erklärungen und Handlungen im Zusammenhang mit Kryptowertpapieren nicht ohne Weiteres mit den Gegebenheiten der Distrtibuted Ledger Technologie in Einklang bringen lassen.

Die in den Materialien in Aussicht gestellte „umfassende Reform des deutschen Wertpapier- und Depotrechts" ${ }^{\text {"89 }}$ ist dringlicher denn je. Sie hat jedenfalls für die am Kapitalmarkt gehandelten Papiere in der Schaffung von Bucheffekten $\mathrm{zu}$ bestehen, die nicht nur eine rechtssichere Übertragung, sondern auch die Transparenz der materiell Berechtigten gewährleisten sollte. ${ }^{90}$ Für Kryptowertpapiere sollte hinsichtlich der den Beteiligten abverlangten Erklärungen und Handlungen wie auch hinsichtlich des Transparenzregimes auf die dezentrale Struktur der Distrtibuted Ledger Technologie Rücksicht genommen werden. ${ }^{91}$

tronischen Wertpapiere, RDi 2021, 161 Rn. 25 ff.; Lebmann, BKR 2020, 431, 437 f.; Linardatos, ZBB 2020, 329, 346f.; Segna, WM 2020, 2301, 2311; Sickinger/Thelen, AG 2020, 862 Rn. 53; wohlwollender Döding/Wentz, WM 2020, 2312, 2321; Mittwoch, WM 2021, 375 ff., 380 f.; Saive, ZRP 2020, 219, 222.

${ }_{88}$ Begr. RegE (Fn. 10), S. 1.

89 Begr. RegE (Fn. 10), S. 40.

90 In diesem Sinne auch die in Fn. 87 angeführten krit. Stimmen.

91 So in Bezug auf Transparenz auch Lehmann, BKR 2020, 431, 438. 



\title{
Eintragung und Publizität elektronischer Wertpapiere
}

\author{
Jan Lieder
}

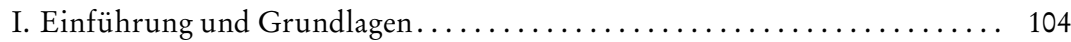

II. Eintragung als elektronischer Skripturakt $\ldots \ldots \ldots \ldots \ldots \ldots \ldots \ldots \ldots . \ldots \ldots$

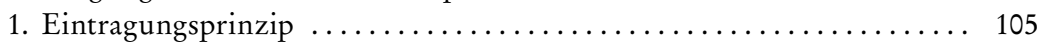

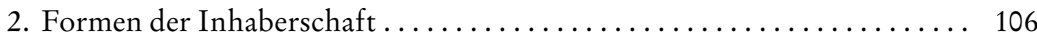

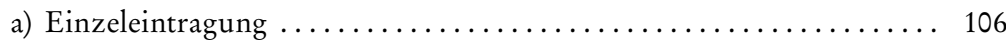

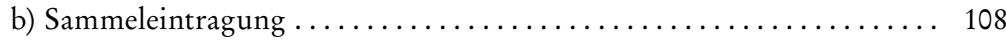

3. Tatbestandliche Voraussetzungen der Registereintragung. . . . . . . . . . 109

a) Aufnahme des Wertpapiers in das Register ................. 109

aa) Rechtsinhalt und Wertpapierkennnummer............... 109

bb) Weitere obligatorische Registerangaben $\ldots \ldots \ldots \ldots \ldots \ldots \ldots . \ldots \ldots$

cc) Zusätzliche Angaben bei Einzeleintragung $\ldots \ldots \ldots \ldots \ldots \ldots \ldots 110$

(1) Rechte Dritter.............................. 111

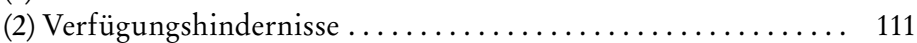

dd) Änderungen des Registerinhalts . . . . . . . . . . . . . . . . 114

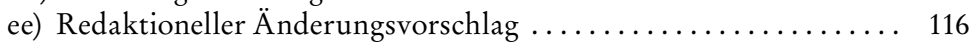

b) Niederlegung der Emissionsbedingungen $\ldots \ldots \ldots \ldots \ldots \ldots \ldots \ldots . \ldots \ldots$

aa) Schutz der Authentizität und Integrität................ 117

bb) Niederlegung statt Eintragung $\ldots \ldots \ldots \ldots \ldots \ldots \ldots \ldots \ldots \ldots . \ldots \ldots$

cc) Zugänglichkeit der Emissionsbedingungen $\ldots \ldots \ldots \ldots \ldots \ldots \ldots 119$

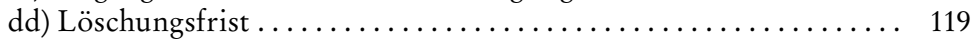

c) Bezugnahme auf Emissionsbedingungen................ 120

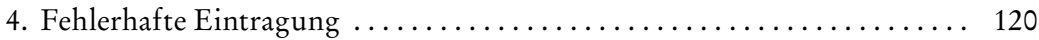

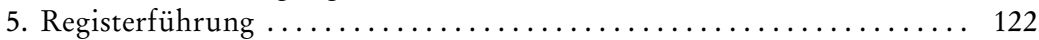

a) Anforderungen an die Registerführung ................. 122

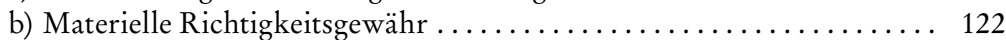

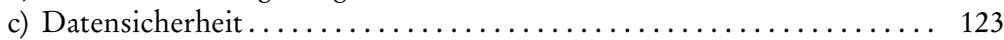

6. Wertpapierrechtlicher Verpflichtungstatbestand ............... 124

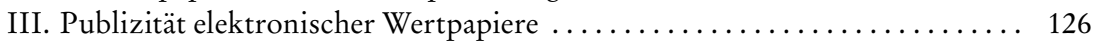

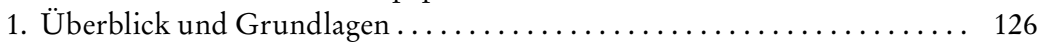

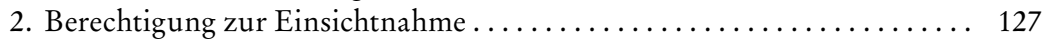

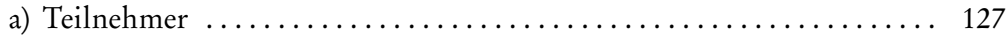

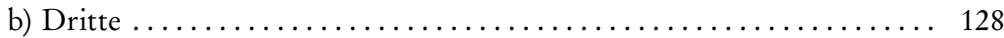

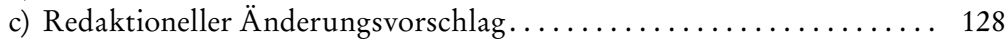

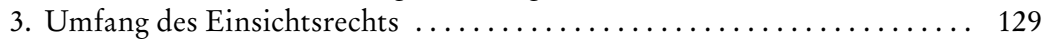

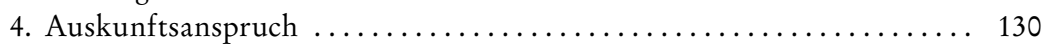

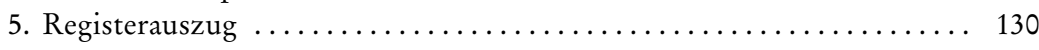




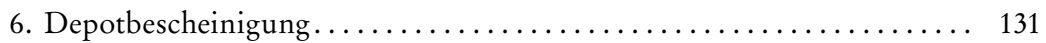

7. Veröffentlichung im Bundesanzeiger.................... 132

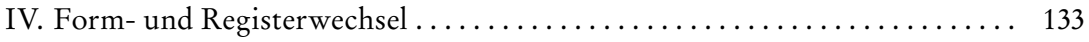

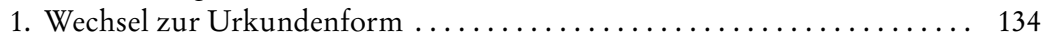

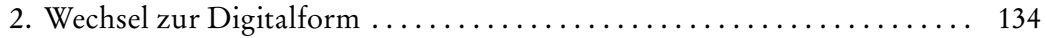

V. Zusammenfassung der wesentlichen Ergebnisse in Thesen . . . . . . . . 135

\section{Einführung und Grundlagen}

Das Proprium elektronischer Wertpapiere liegt nach $\$ 2 \mathrm{Abs.} 1 \mathrm{eWpG}{ }^{1}$ in der Ersetzung der urkundlichen Verbriefung eines körperlichen Wertpapiers durch die Eintragung eines elektronischen Wertpapiers in ein Wertpapierregister. Dessen Primärfunktion besteht in der Gewährleistung von Publizität und Transparenz im überindividuellen Interesse der Sicherheit und Leichtigkeit des Rechts- und Handelsverkehrs. Zugleich bildet die Publizität der Registerangaben die Grundlage für die Beweis- und Gutglaubensfunktion elektronischer Wertpapierregister, geht aber mit ihren unterschiedlichen Facetten deutlich darüber hinaus.

Für die dogmatische Einordnung und die rechtspolitische Analyse der Regelungen über Eintragung und Publizität elektronischer Wertpapiere ist der Grundansatz des Regierungsentwurfs von zentraler Bedeutung, wonach sich die Rechtsnatur elektronischer Wertpapiere nicht grundlegend von traditionellen, in Urkunden verbrieften Wertpapieren unterscheidet. ${ }^{2}$ Stattdessen entfaltet ein elektronisches Wertpapier nach $\$ 2 \mathrm{Abs.} 2 \mathrm{eWpG}$ - abgesehen von den Sonderregelungen des eWpG - dieselbe Rechtswirkung wie ein Wertpapier, das mittels Urkunde begeben worden ist. ${ }^{3}$ Zudem gelangen die Vorschriften und Grundsätze des zivilrechtlichen Wertpapierrechts auf beide Wertpapierarten im Grundsatz unterschiedslos zur Anwendung.

Diese Richtungsentscheidung des Referenten- und Regierungsentwurfs hat aus der Wissenschaft viel Kritik ${ }^{4}$, von Verbandsseite aber auch Zustimmung ${ }^{5}$ er-

${ }^{1}$ Gesetz über elektronische Wertpapiere vom 3.6.2021; vorausgegangen war ein Gesetzentwurf der Bundesregierung, Entwurf eines Gesetzes zur Einführung von elektronischen Wertpapieren, BT-Drucks. 19/26925 vom 24.2.2021; abrufbar unter: https://dip21.bundestag. de/dip21/btd/19/269/1926925 (alle Links in diesem Beitrag wurden zuletzt am 20.05.2021 abgerufen), der nicht unerheblich durch die Beschlussempfehlung und den Bericht des Finanzausschusses, BT-Drucks. 19/29372 (Vorabversion) vom 5.5.2021, modifiziert worden ist.

2 Dazu Begr. RegE, BT-Drucks. 19/26925, S. 37 f.

${ }^{3}$ Kritisch zum Regelungsziel Segna, Elektronische Wertpapiere im zentralen Register, WM 2020, 2301, 2304.

${ }^{4}$ Dubovitskaya, Gesetzentwurf zur Einführung von elektronischen Wertpapieren: ein zaghafter Schritt nach vorn, ZIP 2020, 2551 ff.; Lebmann, Zeitenwende im Wertpapierrecht, BKR 2020, $431 \mathrm{ff}$; Segna, WM 2020, $2301 \mathrm{ff}$.

${ }^{5}$ DAI, Stellungnahme vom 11.9.2020, S. 2 f.; abrufbar unter: https://www.bundesfinanz 
fahren. Es ist hier nicht der Ort, um die Grundsatzkontroverse in allen Einzelheiten auszubreiten und zu analysieren. Stattdessen soll das Eintragungs- und Publizitätssystem auf der Grundlage des vorliegenden Gesetzentwurfs untersucht und gewürdigt werden. Das gilt ungeachtet des Umstands, dass die Kritiker in vielen Punkten Sachgründe auf ihrer Seite haben. Die überwiegende Mehrzahl der hier gefundenen Ergebnisse zu Eintragung und Publizität elektronischer Wertpapiere ließen sich aber auch dann verwerten, wenn sich der Gesetzgeber zur Schaffung echter Wertrechte entschließen sollte.

\section{Eintragung als elektronischer Skripturakt}

Die Eintragung als elektronischer Strukturakt ist konstitutiver Bestandteil des gesamten Lebenszyklus' elektronischer Wertpapiere. Das gilt nicht nur für die Erstbegebung ( $\$ 2$ Abs. 1 S. 2 eWpG), die Übertragung und andere Verfügungen $(\mathbb{S} 24,25 \mathrm{eWpG})$, sondern auch für Verfügungshindernisse ( $\mathbb{S} \mathbb{S}$ Abs.2 S. 1 Nr. 1, S. 3,17 Abs. 2 S. 1 Nr.1, S. 3 eWpG), Rechte Dritter ( $\mathbb{S} 13$ Abs. 2 S. 1 Nr.2, 17 Abs. 2 S. 1 Nr. 2 eWpG), Inhaltsänderungen ( $\$ \mathbb{S} 14$ Abs.1, 18 Abs. 1 eWpG) und das Erlöschen elektronischer Wertpapiere ( $\$ \mathbb{S} 14$ Abs.1, 18 Abs. 1 eWpG). Außerhalb des Registers - etwa durch reine Forderungszession gem. $\$ 398$ BGB - vollzogene Übertragungsvorgänge entfalten hingegen keine Wirkung (vgl. $\$ 24 \mathrm{Nr} .2 \mathrm{eWpG})^{6}$

\section{Eintragungsprinzip}

Insgesamt fungiert das Wertpapierregister weniger als Transaktionsregister, aus dem sich - im Stile einer Blockchain - lediglich die Erwerbsketten und eingetragenen Änderungen der Reihe nach abrufen lassen. Stattdessen sind aus dem Register stets die aktuellen Informationen zu den eingetragenen Wertpapieren ersichtlich. Diese Darstellungsart entspricht - ebenso wie bei Grundbuch und Handelsregister - dem Realfoliensystem ${ }^{7}$ und geht damit über die durch Blockchain-Datenbanken gewährleistete Dokumentationsfunktion hinaus. ${ }^{8}$

ministerium.de/Content/DE/Gesetzestexte/Gesetze_Gesetzesvorhaben/Abteilungen/Abtei lung_VII/19_Legislaturperiode/2020-08-11-einfuehrung-elektronische-wertpapiere/Stel lungnahme-da.pdf?_blob=publicationFile\&v $=1$.

${ }_{6}$ Begr. RegE, BT-Drucks. 19/26925, S.64; für Einzelheiten siehe Omlor, Verfügungen über elektronische Wertpapiere, $\$ 6$, in diesem Band.

$7 \mathrm{Zu}$ diesem Aspekt mit Blick auf ein Blockchain-Grundbuch näher Hecht, Notariat 4.0 und Blockchain-Technologie, MittBayNot 2020, 314, 318; zum Realfoliensystem allgemein Bauer/Schaub/Waldner, GBO (4. Aufl. 2018), $\$ 3$ Rn. 7; BeckOK/Holzer, GBO (41. Ed. Stand: 1.2.2021), \$3 Rn. 1; Schön/Stöber, Grundbuchrecht (16. Aufl. 2020), 1. B. Rn. 80.

8 Vgl. Sickinger/Thelen, Anleihen und Genussscheine auf die Blockchain, AG 2020, 862 Rn. 22; kritisch zu diesem Aspekt Dubovitskaya, ZIP 2020, 2551, 2559; Lehmann, BKR 2020, 
Das Eintragungsprinzip 9 und die ihm zugrunde liegende konstitutive Wirkung elektronischer Eintragungen schafft eine belastbare Grundlage für die Dokumentation der an einem elektronischen Wertpapier bestehenden Rechtsverhältnisse und schützt so das Vertrauen auf die materielle Richtigkeit und Vollständigkeit des Wertpapierregisters. Rechtsgeschäftliche Änderungen am Wertpapier werden danach erst mit ihrer Eintragung wirksam. Umgekehrt sind Rechtsänderungen, die sich außerhalb des Wertpapierregisters vollziehen, nahe$\mathrm{zu}$ ausgeschlossen. ${ }^{10}$ In der Konsequenz führt das - bei Einzeleintragung ${ }^{11}-\mathrm{zu}$ einem Gleichlauf von materieller Berechtigung und formeller Inhaberschaft. ${ }^{12}$ Erst die konstitutive Wirkung verleiht dem elektronischen Wertpapierregister dasjenige Maß an materieller Richtigkeit und Verlässlichkeit, das in rechtspolitischer Hinsicht für die Zulassung des Gutglaubenserwerbs nach $\$ 26$ eWpG und den Entzug des Forderungsrechts beim wahren Berechtigten zwingend erforderlich ist. ${ }^{13}$ Das Eintragungsprinzip bildet insofern die Legitimationsgrundlage für die Vermutungswirkung und den Gutglaubenserwerb im elektronischen Wertpapierrecht.

\section{Formen der Inhaberschaft}

Was für mittels Urkunde begebene Wertpapiere die Verbriefung in Einzelurkunden einerseits und Sammel- oder Globalurkunden andererseits, ist für elektronische Wertpapiere nach Maßgabe des $\$ 8$ Abs. 1 eWpG die Einzel- und Sammeleintragung in das zentrale oder das Kryptowertpapierregister.

\section{a) Einzeleintragung}

Bei der Einzeleintragung, die sich vor allem an den privaten Kapitalmarkt richtet, wird als Inhaber eine natürliche oder juristische Person oder eine rechtsfähige Personengesellschaft in das Register eingetragen, die das elektronische Wertpapier als Berechtigte hält ( $\$ 8$ Abs. 1 Nr.2 eWpG). Das kann man aus rechtskonstruktiver Perspektive als den systematischen Grundfall bezeichnen, weil sich hier die formelle Inhaberschaft und die materielle Berechtigung an

431, 436; vgl. weiter Kleinert/Mayer, Elektronische Wertpapiere und Krypto-Token, EuZW $2019,857,858$.

${ }^{2}$ Zum Eintragungsprinzip im Immobiliarsachenrecht ausf. Lieder, Die rechtsgeschäftliche Sukzession (2015), S. $370 \mathrm{ff}$.

${ }^{10}$ Für wenige Ausnahmen siehe unten II. 3. a).

11 Siehe sogleich unten II. 2. a).

12 Vgl. (zum Grundstücksrecht) MüKo/Kohler, BGB (8. Aufl. 2020), vor $\$ 873$ Rn.14; Lieder, Die rechtsgeschäftliche Sukzession (2015), S. 377; siehe ferner Kleinert/Mayer, EuZW 2020, 1059, 1062.

${ }^{13}$ Vgl. (zum Grundstücksrecht) Lieder, Die Lehre vom unwirksamen Rechtsscheinträger: Ein Beitrag zu den rechtsdogmatischen und rechtsökonomischen Grundlagen des redlichen Erwerbs, AcP 210 (2010), 857, 870; Lieder, Die rechtsgeschäftliche Sukzession (2015), S. 377. 
dem Recht aus dem Wertpapier decken, insbesondere kein Intermediär zwischengeschaltet ist. In der Praxis ist hingegen die massenhafte Emission der Regelfall, ${ }^{14}$ so dass die Einzeleintragung elektronischer Wertpapiere auf keine größere wirtschaftliche Relevanz wird hoffen dürfen.

Dennoch verdient es aus rechtspolitischer Perspektive durchaus Beifall, dass die Einzeleintragung zulassen wird, und zwar - anders als noch im Referentenentwurf (vgl. $\$ 8$ Abs. 2 eWpG-RefE) - nicht nur für Kryptowertpapiere, sondern auch für Zentralregisterwertpapiere (vgl. $\$ 8$ Abs. 1 Nr. 2 eWpG). Mag die praktische Notwendigkeit für die Einzeleintragung im zentralen Wertpapierregister auch tendenziell gering sein, so sind doch praktische Anwendungsfälle denkbar, in denen die Beteiligten an einer Einzeleintragung von Zentralregisterwertpapieren interessiert sind. Man denke etwa an die Konstellation, dass sich an einer Emission nur institutionelle Anleger beteiligen und von den Parteien auch kein Handel der Wertpapiere auf einem Zweitmarkt beabsichtigt ist. ${ }^{15}$ Zudem erhöht es die Gestaltungsfreiheit der Beteiligten, wenn Einzel- und Sammeleintragungen unterschiedslos für zentrale und Kryptowertpapierregister in Betracht kommen. Zwingende Sachgründe für den Ausschluss einer bestimmten Gestaltungsform sind jedenfalls nicht ersichtlich.

Erfreulich ist, dass der Finanzausschuss den - auch vom Verf. vorgetragenen-Vorschlag aufgegriffen hat, den Gesetzestext dahingehend zu korrigieren, dass nicht nur natürliche und juristische Personen als Inhaber in das Register eingetragen werden können, sondern auch rechtsfähige Personengesellschaften. Für den Inhaber eines Wertpapiers kann nichts anderes gelten als für die registerführende Stelle, für welche neben natürlichen und juristischen Personen auch „rechtsfähige Personengruppen“, sprich rechtsfähige Personengesellschaften ${ }^{16}$, in Betracht kommen. ${ }^{17}$ Mit Blick auf die Modernisierung des Personengesellschaftsrechts muss für die BGB-Gesellschaft weiterhin sichergestellt werden, dass auch Angaben zu deren Gesellschafterkreis und Vertretungsbefugnissen erkennbar sind. Sowohl in der Endfassung des eWpG als auch des $\mathrm{MoPeG}^{18}$ ist allerdings versäumt worden, die beiden Neuregelungen miteinander abzustimmen und zu verzahnen. In Anlehnung an $\$ 67 \mathrm{Abs} .1 \mathrm{~S} .3 \mathrm{AktG}-\mathrm{MoPeG}$ und $\ 40$ Abs. 1 S. 3 GmbHG-MoPeG sollte in $\$ 8$ Abs. 1 Nr. 2 eWpG noch folgender Satz aufgenommen werden:

14 Vgl. nur MüKo/Habersack, BGB (8. Aufl. 2020), vor \$793 Rn. 32 f.; Lebmann, Finanzinstrumente (2009), S.346.

15 So auch Kleinert/Mayer, EuZW 2020, 1059, 1061; Linardatos, Elektronische Schuldverschreibungen auf den Inhaber - des Wertpapiers neue Kleider, ZBB 2020, 329, 336.

16 Vgl. auch Döding/Wentz, Der Referentenentwurf zur Einführung von elektronischen Wertpapieren und Kryptowertpapieren, WM 2020, 2312, 2319 m. Fn. 141.

17 Begr. RegE, BT-Drucks. 19/26925, S. 59.

18 Gesetz zur Modernisierung des Personengesellschaftsrecht (Personengesellschaftsmodernisierungsgesetz - MoPeG) vom 10.8.2021. 
„Eine Gesellschaft bürgerlichen Rechts kann nur in das elektronische Wertpapierregister eingetragen und Veränderungen können nur vorgenommen werden, wenn sie in das Gesellschaftsregister eingetragen ist.“

\section{b) Sammeleintragung}

Bei der Sammeleintragung ${ }^{19}$ ist im Register ausgewiesener Inhaber des elektronischen Wertpapiers eine Wertpapiersammelbank oder ein Verwahrer ( $\$ 8$ Abs. 1 Nr. 1 eWpG). Sie halten für die Miteigentümer am Sammelbestand als materiell Berechtigte den nach $\$ 2$ Abs. 3 eWpG fingierten unmittelbaren Besitz am sammeleingetragenen Wertpapier. ${ }^{20}$ Fungiert die als Inhaber eingetragene Wertpapiersammelbank zugleich als registerführende Stelle, entspricht die elektronische Sammeleintragung dem heute unter Verwahrung von Dauerglobalurkunden praktizierten Effektengiroverkehr. ${ }^{21} \mathrm{Da}$ in der rein elektronischen Umsetzung der funktional vergleichbaren Vorgänge auf die Verwahrung von Urkunden verzichtet werden kann, darf man sich von der Sammeleintragung gewisse Effizienzgewinne wegen wegfallender Transaktionskosten erhoffen.

Der sachenrechtliche Regulierungssatz schlägt weiterhin bei der rechtlichen Qualifikation elektronischer Wertpapiere in Sammeleintragung durch. Sie gelten nach $₫ 9$ Abs. 1 eWpG als Wertpapiersammelbestand und die Berechtigten als Miteigentümer nach Bruchteilen nach $\$ \$ \$ 741 \mathrm{ff}$. BGB am elektronischen Wertpapier, und zwar im Umfang des Nennbetrags der für den jeweiligen Berechtigten in Sammeleintragung gehaltenen Rechte. Diese Rechtsstellung erlaubt ihnen im Insolvenzfall die Aussonderung ihrer Anteile nach $\$ 47 \mathrm{InsO}^{22}$ und deren Verteidigung gegen Vollstreckungsmaßnahmen von Drittgläubigern mittels Drittwiderspruchsklage gem. $\$ 771$ ZPO. ${ }^{23}$ Auch wenn es sich bei den Rechten aus dem Wertpapier weiterhin um Forderungen und nicht um bewegliche Sachen handelt, lässt sich mit Blick auf den vorbezeichneten insolvenzund vollstreckungsrechtlichen Schutz durchaus von einer Verdinglichung dieser Forderungen sprechen. ${ }^{24}$ Das zeigt sich auch im Zusammenhang mit der Verfügung über sammeleingetragene Wertpapiere, die sich nach sachenrechtlichen Grundsätzen ( $\$ \int 929 \mathrm{ff}$. BGB) vollzieht. ${ }^{25}$

19 Die am Begriff der Sammeleintragung geäußerte Kritik (Lehmann, BKR 2020, 431, 436) erscheint ein wenig überzogen, weil sich diese Form der Inhaberschaft zumindest auf eine Mehrheit von Forderungsrechten bezieht, die in Form der Sammeleintragung zusammengefasst werden (so auch Linardatos, ZBB 2020, 329, 335 f.).

${ }^{20}$ Dazu und zum Folgenden Begr. RegE, BT-Drucks. 19/26925, S. 55; für unberechtigte Kritik an der Terminologie vgl. Lehmann, BKR 2020, 431, 436.

${ }_{21}$ Zur Einbeziehung elektronischer Schuldverschreibungen in den Effektengiroverkehr näher Segna, WM 2020, 2301, 2307.

22 Dazu näher Sickinger/Thelen, AG 2020, 862 Rn. 15.

23 Segna, WM 2020, 2301, 2307.

${ }^{24}$ In diesem Sinne auch Linardatos, ZBB 2020, 329, 332; Segna, WM 2020, 2301, 2308.

25 Dazu Omlor, Verfügungen über elektronische Wertpapiere, $\$ 6$, in diesem Band; vgl. weiter Linardatos, ZBB 2020, 329, 332 f., 340 ff.; Segna, WM 2020, 2301, 2308 f. 
Ohne eine eigene materielle Berechtigung am elektronischen Wertpapier zu besitzen, fungieren die Wertpapiersammelbank oder ein Verwahrer nach $\$ 9$ Abs. 2 eWpG als Ermächtigungstreuhänder. ${ }^{26}$ Sie sind also keine Vollrechtsinhaber, sondern verfügen nach $\$ 185$ BGB über die zur Verwaltung notwendige Rechtsmacht über das Treugut. In dieser Funktion nimmt die Bank die aus dem Wertpapier folgenden Rechte für den materiell Berechtigten wahr. ${ }^{27}$ Insbesondere kann sie die Rechte im eigenen Namen gegen den Emittenten geltend machen. Die notwendige Legitimationswirkung folgt aus dem - $\$ 793$ Abs. 1 S. 1 BGB nachgebildeten $-₫ 28$ Abs. 1 S. 1 eWpG. Darüber hinaus ordnet $₫ 9 \mathrm{~b}$ Abs. 1 DepotG an, dass für elektronische Inhaberschuldverschreibungen, die in Form einer Sammeleintragung vom Verwahrer auf einem Depotkonto des Hinterlegers verbucht werden, die Bestimmungen über Sammelverwahrung und Sammelbestand sinngemäße Anwendung finden.

\section{Tatbestandliche Voraussetzungen der Registereintragung}

Die Eintragung eines elektronischen Wertpapiers verlangt nach Maßgabe des \4 Abs. 4 eWpG (1.) die Aufnahme gesetzlich notwendiger Angaben in ein elektronisches Wertpapierregister, (2.) die Niederlegung der Emissionsbedingungen bei der registerführenden Stelle und (3.) eine Bezugnahme auf die niedergelegten Bedingungen.

\section{a) Aufnabme des Wertpapiers in das Register}

Von zentraler Bedeutung ist die Aufnahme der nach $\$ 13$ oder $\$ 17 \mathrm{eWpG}$ erforderlichen Registerangaben in das elektronische Wertpapierregister. Die Angaben dienen der (sachenrechtlichen) Bestimmbarkeit des Wertpapiers und nehmen im Interesse eines effektiven Schutzes des redlichen Rechtsverkehrs an der Publizitätswirkung des Wertpapierregisters teil. ${ }^{28}$ Die Registerangaben zielen darauf ab, dass sich der Rechtsverkehr auf einen Blick über die wesentlichen Merkmale des Wertpapiers informieren kann. ${ }^{29}$ Bei der Eintragung in das Kryptowertpapierregister muss zudem deutlich werden, dass es sich nicht nur um eine bloße Forderung oder andere private Rechte handelt, die auf einer Blockchain gehandelt werden, sondern um ein elektronisches Wertpapier. ${ }^{30}$

\section{a) Rechtsinbalt und Wertpapierkennnummer}

Die Eintragung hat Art und Inhalt des Rechts in seinen wesentlichen Grundzügen zu beschreiben. Mit Blick auf den limitierten Anwendungsbereich wird bei einer Inhaberschuldverschreibung der jeweilige Leistungsanspruch gegen

\footnotetext{
26 Vgl. Dubovitskaya, ZIP 2020, 2551, 2556; Segna, WM 2020, 2301, 2307.

27 Vgl. auch Segna, WM 2020, 2301, 2307.

28 Vgl. Begr. RegE, BT-Drucks. 19/26925, S. 40 f.

29 Begr. RegE, BT-Drucks. 19/26925, S. 53, 59.

30 Vgl. Begr. RegE, BT-Drucks. 19/26925, S. 59.
} 
den Emittenten näher zu spezifizieren sein. Bei einer Geldforderung ist deren Höhe, Zins und Fälligkeit anzugeben. ${ }^{31}$ Zur eindeutigen Bestimmung des verkörperten Rechts ist die Angabe der individuellen Wertpapierkennnummer von zentraler Bedeutung, die für herkömmliche Wertpapiere derzeit von der Herausgebergemeinschaft Wertpapiermitteilungen, Keppler, Lehmann GmbH \& Co. KG vergeben wird. Ob diese Gesellschaft auch für die Vergabe von Kennnummern für elektronische Wertpapiere verantwortlich zeichnen wird, lässt der Entwurf offen und verlässt sich auf die Selbstorganisationskräfte des Rechtsverkehrs. ${ }^{32}$ Maßgeblich ist in jedem Fall, dass die vergebene Wertpapierkennnummer von jedem Berechtigten dauerhaft abgerufen und mittels elektronischer Hilfsmittel wahrgenommen werden kann.

\section{bb) Weitere obligatorische Registerangaben}

$\mathrm{Zu}$ den obligatorischen Registerangaben zählt weiterhin das Emissionsvolumen entsprechend der Emissionsbedingungen. Im Fall der Aufstockung ist das weitere Emissionsvolumen unter Angabe der Wertpapierkennnummer anzugeben. Erfolgt eine Höchstbetragsemission, sind das gegenwärtige Volumen und der Maximalbetrag anzugeben. ${ }^{33}$ Hinzu kommt der Nennbetrag des auf den Inhaber entfallenden Teils der Inhaberschuldverschreibung oder aber die Angabe, dass es sich um ein nennbetragsloses Wertpapier handelt. Zudem ist der Emittent - bei einer Schuldverschreibung also der Schuldner - möglichst unter Bezeichnung des Legal Entity Identifier (LEI) einzutragen. Erforderlich ist ferner die Kennzeichnung als Einzel-oder Sammeleintragung. ${ }^{34}$ Schließlich sind noch der Inhaber des elektronischen Wertpapiers zu bezeichnen und - soweit einschlägig - Angaben zu einem Mischbestand nach $\$ 9$ Abs. 3 eWpG zu machen. Gemeinsam mit den sogleich ${ }^{35}$ zu erläuternden zusätzlichen Angaben bei der Einzeleintragung hat die registerführende Stelle sicherzustellen, dass sämtliche Angaben in einer Weise verknüpft sind, dass sie nur zusammen abgerufen werden können ( $\$ 13 \mathrm{Abs.3,} \$ 17 \mathrm{Abs.3} \mathrm{eWpG}$ ).

\section{cc) Zusätzliche Angaben bei Einzeleintragung}

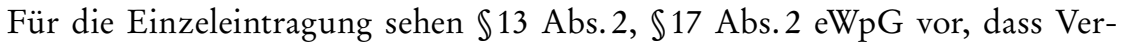
fügungsbeschränkungen (Nr.1) und Rechte Dritter (Nr.2) in elektronische Wertpapierregister eingetragen werden.

\footnotetext{
31 Begr. RegE, BT-Drucks. 19/26925, S. 54.

32 Begr. RegE, BT-Drucks. 19/26925, S. 40 f.

33 Begr. RegE, BT-Drucks. 19/26925, S. 54.

34 Siehe nochmals oben II. 2.

35 Siehe unten II. 3. a) cc).
} 


\section{(1) Rechte Dritter}

Die Eintragungsfähigkeit für die an einem elektronischen Wertpapier bestehenden Rechte Dritter steht in untrennbarer Verbindung mit der Möglichkeit, nach $\$ 24 \mathrm{eWpG}$ über Rechte an elektronischen Wertpapieren zu verfügen. Die Notwendigkeit der Eintragung ergibt sich für Rechte Dritter bereits aus dem Umstand, dass sie konstitutiven Charakter hat und damit Wirksamkeitsvoraussetzung ist. Zudem besteht die Gefahr, dass ohne ordnungsgemäße Eintragung das Wertpapier nach $\$ 26 \mathrm{~S} .1 \mathrm{eWpG}$ gutgläubig lastenfrei erworben werden kann. Das gilt namentlich für die Belastung von Inhaberschuldverschreibungen mit einem Nießbrauch oder Pfandrecht.

Die Erstreckung der Publizitäts- und Gutglaubenswirkung auf Rechte Dritter ist aus dem Sachenrecht bekannt (vgl. \$892 Abs. 1 S. 1, \$936 BGB) und hat sich dort bestens bewährt. Demgegenüber ist die mangelnde Publizität an $\mathrm{GmbH}$-Geschäftsanteilen bestehender Rechte Dritter im Kontext des $\$ 16$ Abs. 3 GmbHG Gegenstand anhaltender Kritik und von Reformvorschlägen. ${ }^{36}$ In der Sache hat die Zulassung des redlichen lastenfreien Erwerbs den Vorzug innerer Folgerichtigkeit für sich: Wenn nämlich das Vollrecht durch den Redlichen wirksam erworben werden und so der totale Rechtsmangel des Veräußerers kraft guten Glaubens überwunden werden kann, dann muss erst recht der teilweise Rechtsmangel (Belastung) durch eine Gutglaubensvorschrift für unbeachtlich erklärt werden können. Die mit der Publizität von Rechten Dritter verbundene Erhöhung des Publizitätsniveaus und Verkehrsschutzes ist daher uneingeschränkt zu begrüßen.

\section{(2) Verfügungsbeschränkungen}

Besonderes Augenmerk gilt der Eintragungsfähigkeit von Verfügungshindernissen. Nach der Regierungsbegründung sollten darunter Verfügungsverbote und Verfügungsbeschränkungen, aber auch Beschränkungen der Geschäftsfähigkeit zu verstehen sein. ${ }^{37}$ Durch die Eintragung der Geschäftsfähigkeit sollte der gesetzliche Vertreter das Wertpapier gewissermaßen sperren können, weil eine Umtragung durch die registerführende Stelle ohne seine Mitwirkung nicht erfolgen dürfe. Anders als bei eingetragenen Rechten Dritter sollte ein redlicher einwendungsfreier Erwerb nach $\$ 26 \mathrm{eWpG}$-RegE allerdings in allen Fällen von Verfügungshindernissen ausscheiden. ${ }^{38}$ Das bedeutete eine wesentliche Abweichung von der noch im Referentenentwurf enthaltenen - deutlich weitergehenden - Regelung des $\$ 26$ Nr. 3 bis 5 eWpG-RefE. Danach sollte der

36 Vgl. Lutter/Hommelhoff/Bayer, GmbHG (20. Aufl. 2020), \$16 Rn.74; Harbarth/ Friedrichson, $10 \mathrm{Jahre} \mathrm{MoMiG} \mathrm{-} \mathrm{Welche} \mathrm{Impulse} \mathrm{benötigt} \mathrm{das} \mathrm{GmbH-Recht} \mathrm{heute?,} \mathrm{GmbHR}$ 2018, 1174, 1177f.; Lieder, Die rechtsgeschäftliche Sukzession (2015), S. $629 \mathrm{ff}$.

37 Begr. RegE, BT-Drucks. 19/26925, S. 55.

38 Begr. RegE, BT-Drucks. 19/26925, S. 66. 
Veräußerer als verfügungsbefugt ${ }^{39}$ und geschäftsfähig, ein Vertreter als bevollmächtigt und geschäftsfähig gelten. ${ }^{40} \mathrm{Im}$ Falle einer fehlenden Eintragung solcher Verfügungshindernisse kam ein gutgläubiger einwendungsfreier Wertpapiererwerb in Betracht.

Dass der Regierungsentwurf auf einen Gutglaubensschutz für mangelnde Geschäftsfähigkeit und Bevollmächtigung verzichtet, ist mit Blick auf die Grundwertungen des Zivilrechts uneingeschränkt zu begrüßen. ${ }^{41}$ Zunächst kommt dem Minderjährigenschutz im gesamten Privatrecht ein besonders hohes Schutzniveau zu. ${ }^{42}$ Erwägungen des Verkehrsschutzes vermögen sich demgegenüber nicht durchzusetzen, weil (teilweise) Geschäftsunfähige hier für eine Nachlässigkeit des gesetzlichen Vertreters zur Verantwortung gezogen werden und zudem der Umstand der Geschäftsunfähigkeit praktisch häufig erst spät entdeckt wird, so dass eine etwaige Eintragung der mangelnden Geschäftsfähigkeit dem Betroffenen regelmäßig wenig nutzen wird. ${ }^{43}$ Davon abgesehen genießt der gute Glaube an die uneingeschränkte Geschäftsfähigkeit ${ }^{44} \mathrm{im}$ Bürgerlichen Recht ebenso wenig Schutz wie - ungeachtet der Spezialtatbestände

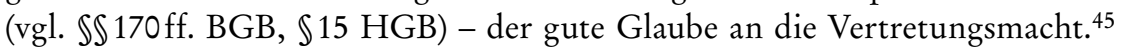
Von diesem Prinzip für elektronische Wertpapiere abzuweichen, erscheint aus rechtspolitischer Perspektive nicht angezeigt.

Sachlich überzeugend ist es allerdings, dass der Gesetzgeber einen - vom Verf. vorgetragenen - Vorschlag aufgegriffen und Verfügungsbeschränkungen zugunsten einer bestimmten Person einer gesonderten Regelung zugeführt und zugleich nach Maßgabe des $\$ 26$ S. 2 eWpG einen redlichen, verfügungsbeschränkungsfreien Erwerb angeordnet hat. Zur Begründung hatte der Verf. in seinem Referat bereits auf $\$ 892$ Abs. 1 S. 2 BGB verwiesen und eine Anpassung des $\$ 26 \mathrm{eWpG}$ dahingehend gefordert, dass der Erwerber im Hinblick auf solche Verfügungsbeschränkungen auf die Vollständigkeit des Wertpapierregisters vertrauen darf und trotz bestehender Beschränkung das eingetragene Wertpapier - redlich einwendungsfrei - erwirbt, soweit die Beschränkung nicht

39 Zustimmend zum RefE noch Sickinger/Thelen, AG 2020, 862 Rn. $31 \mathrm{f}$.

40 Kritisch zur Ausdehnung des Gutglaubensschutzes auf Vertretungsmacht und Geschäftsfähigkeit Dubovitskaya, ZIP 2020, 2551, 2559; Sickinger/Thelen, AG 2020, 862 Rn. 33 ff., 37 f.; ebenso zur Geschäftsfähigkeit Lebmann, BKR 2020, 431, 437.

41 A. A. Omlor, Verfügungen über elektronische Wertpapiere, $\$ 6 \mathrm{II} 4 \mathrm{e}$, in diesem Band.

${ }^{42} \mathrm{Dazu}$ allgemein BeckOK/Wendtland, BGB (Stand: 1.2.2021), \$106 Rn. 1; Staudinger/ Klumpp, BGB (2017), \$106 Rn. 1; MüKo/Spickhoff, BGB (8. Aufl. 2018), \$106 Rn. 1; Erman/ Müller, BGB (16. Aufl. 2020), \$106 Rn.1.

43 Vgl. Linardatos, ZBB 2020, 329, $344 \mathrm{f}$.

${ }^{44}$ Vgl. BGH, NJW 1977, 622, 623; MüKo/Spickhoff, BGB (8. Aufl. 2018), $\$ 106$ Rn. 17; Staudinger/Klumpp, BGB (2017), $\$ 106$ Rn. 7 aE.

${ }^{45}$ Vgl. Linardatos, ZBB 2020, 329, 343; siehe allgemein MüKo/Schubert, BGB (8. Aufl. 2018), \$170 Rn.1; BeckOK/Schäfer, BGB (Stand: 1.2.2021), \$170 Rn. 1; Staudinger/Schilken, BGB (2019), $\$ 170$ Rn.1. 
eingetragen und der Erwerber in gutem Glauben ist. Diesem Ansatz ist der Finanzausschuss gefolgt. ${ }^{46}$

In diesem Kontext erweisen sich die erfassten Beschränkungen als sehr heterogene Gruppe, die sich aber allesamt durch ihre Anfälligkeit für einen redlichen Erwerb auszeichnen, der sich aus einer positivrechtlichen Anordnung ergeben muss. ${ }^{47}$ Das gilt namentlich für $\int \mathbb{S} 135$ Abs. 2, 136, 1984 Abs. 1 S. 2, 2113 Abs. 3, 2211 Abs. 2 BGB, $\iint 81$ Abs. 1 S.2, 91 Abs. 2 InsO, $\$ 938$ Abs. 2 ZPO. ${ }^{48}$ Folgt man der hier vertretenen Auffassung und schützt den guten Glauben an die Abwesenheit solcher Verfügungsbeschränkungen, ${ }^{49}$ dann müssen die auf Grundstücksrechte bezogenen Regelungen um eine Bestimmung zur Verfügung über elektronische Wertpapier ergänzt werden.

Nicht erfasst werden hingegen absolut wirkende Verfügungsbeschränkungen, namentlich solche des ehelichen Güterrechts oder des Vormundschaftsrechts (vgl. IS 1365,1423 ff., $1812 \mathrm{ff}$. BGB). ${ }^{50}$ Mit Blick auf ihren Schutzzweck können sie kraft guten Glaubens des Erwerbers im Liegenschaftsrecht nicht überwunden werden. Es ist kein sachlicher Grund dafür ersichtlich, weshalb dies mit Blick auf elektronische Wertpapiere anders sein sollte. Das gilt umso mehr, als das Wertpapierregister mit keiner ebenso starken Richtigkeitsgewähr ausgestattet ist wie das Grundbuch. Zur Gewährleistung der Übersichtlichkeit und Klarheit der Registerangaben erscheint auch die Eintragung solcher Verfügungsbeschränkungen - entgegen der Einlassungen der Regierungsbegründung 51 - nicht opportun. Diese Position hat sich zwischenzeitlich auch der Finanzausschuss zu eigen gemacht und absolut wirkende Verfügungsbeschränkungen aus dem Anwendungsbereich der $₫ 13$ Abs. 2 S. 1 Nr. 1, $\mathbb{1 7}$ Abs. 2 S. 1 Nr. 1 eWpG ausgeklammert. Vollkommen zu Recht hat der Ausschuss weiterhin - in Übereinstimmung mit dem Referat des Verf. - klargestellt, dass auch Beschränkungen der Geschäftsfähigkeit und Mängel der Vertretungsmacht nicht unter den Begriff der Verfügungsbeschränkungen zu subsumieren sind. ${ }^{52}$

Wenn Mängel der Geschäftsfähigkeit und absolut wirkende Verfügungsbeschränkungen für die Gutglaubenswirkung des Wertpapierregisters ohne Belang sind (vgl. \$26 S. 3 eWpG), dann stellt sich aber die Frage, weshalb darauf be-

\footnotetext{
46 Finanzausschuss, BT-Drucks. 19/29372, S. 56f., 60.

47 So auch Staudinger/Picker, BGB (2019), \$892 Rn. 240.

48 Vgl. nur MüKo/Kohler, BGB (8. Aufl. 2020), \$892 Rn. 59f.; Erman/Artz, BGB (16. Aufl. 2020), \892 Rn.41; Staudinger/Picker, BGB (2019), \$892 Rn.240. - Aufgegriffen nun vom Finanzausschuss, BT-Drucks. 19/29372, S. 56.

49 Dagegen Linardatos, ZBB 2020, 329, 345 speziell mit Blick auf $₫ 80$ InsO.

50 Für Einzelheiten vgl. Staudinger/Picker, BGB (2019), \$892 Rn. 265 ff.; MüKo/Kohler, BGB (8. Aufl. 2020), \$892 Rn. 59; Erman/Artz, BGB (16. Aufl. 2020), $\$ 892$ Rn. 42; BeckOK/ Eckert, BGB (Stand: 1.2.2021), $\$ 892$ Rn.16. - Aufgegriffen nun vom Finanzausschuss, BTDrucks. 19/29372, S. 57.

51 Begr. RegE, BT-Drucks. 19/26925, S. 55.

52 Finanzausschuss, BT-Drucks. 19/29372, S. 57.
} 
zogene Informationen nach Maßgabe der $₫ 13$ Abs. 2 S. 3, $\$ 17$ Abs. 2 S. 3 eWpG dennoch auf Weisung von Weisungsberechtigten i.S.d. $\$ 14$ Abs. 1 S. 1 eWpG in das Register eingetragen werden können. Für das Immobiliarsachenrecht ist man sich einig, dass persönliche Verhältnisse des Eingetragenen im Grundbuch gerade nicht verlautbart werden. ${ }^{53}$ Das gilt für die Rechts- und Geschäftsfähigkeit $^{54}$ ebenso wie für die Vertretungsmacht ${ }^{55}$ und die Verfügungsbefugnis. ${ }^{56}$ Nicht zuletzt der Grundsatz der Übersichtlichkeit und Klarheit des Registers spricht dafür, auf die Eintragung solcher Tatsachen zu verzichten, zumal sie - zu Recht - auch nicht an der Vermutung der Richtigkeit und Vollständigkeit des Registers teilnehmen.

Die vom Finanzausschuss gegebene Begründung, die Eintragungen hätten eine Informations- und Warnfunktion und der Erwerber werde nach Einsichtnahme in das Register von einem Erwerb absehen, ${ }^{57}$ kann schwerlich überzeugen. Denn der Erwerber ist vor Durchführung einer Transaktion gerade nicht verpflichtet, sich durch Einsichtnahme in das elektronische Wertpapierregister von einer Abwesenheit entsprechender Beschränkungen zu überzeugen. In Übereinstimmung mit dem Grundbuch ${ }^{58}$ und dem Handelsregister ${ }^{59}$ vermittelt das elektronische Wertpapierregister keinen konkreten, sondern einen abstrakten Vertrauensschutz. Davon abgesehen erscheint es wenig schlüssig, wenn die registerführende Stelle zwar nicht verpflichtet sein soll, die Angaben, die sie auf Weisung in das Register eintragen muss, auf ihre Richtigkeit zu prüfen, umgekehrt aber daran mitwirken soll, dass keine Eintragungen eines (vermeintlichen) Erwerbers in das Wertpapierregister gelangen. ${ }^{60}$

\section{dd) Änderungen des Registerinhalts}

Änderungen der Registerangaben nach $\$ 13$ Abs. 1 und Abs. 2 eWpG sowie die Löschung des Wertpapiers und der niedergelegten Emissionsbedingungen nimmt die registerführende Stelle nach Maßgabe der $\mathbb{} 14$ Abs. $1, \mathbb{S} 18$ Abs. 1 eWpG nur aufgrund einer Weisung vor. Für die Ausführung mehrerer Weisun-

53 Vgl. RGZ 69, 263, 268; 84, 242, 245; 88, 83, 89; 134, 283; OLG Düsseldorf FGPrax 2017, 35, 36; MüKo/Kobler, BGB (8. Aufl. 2020), \892 Rn. 15; Staudinger/Picker, BGB (2019), $\$ 892$ Rn. 67.

54 RGZ 69, 263, 268.

55 KG OLGE 18, $110 \mathrm{f}$.

56 RGZ 84, 242, 245.

57 Finanzausschuss, BT-Drucks. 19/29372, S. 57.

58 Vgl. RGZ 86, 353, 356; BGH NJW 1980, 2413, 2414; NJW-RR 2013, 789 Rn. 15; Staudinger/Gursky, BGB (2019), \892 Rn. 7; MüKo/Kohler, BGB (8. Aufl. 2020), \892 Rn. 2, 4, 45; ausf. J. Hager, Verkehrsschutz durch redlichen Erwerb, 1990, S. $419 \mathrm{ff}$.

59 Vgl. BGHZ 65, 309, 311; Bayer/Lieder, Handels- und Gesellschaftsrecht (2. Aufl. 2021), Rn. 83; Schilken, Abstrakter und konkreter Vertrauensschutz im Rahmen des $\$ 15$ HGB, AcP 187 (1987), 1, 6 f.; verallgemeinernd Lieder, Die rechtsgeschäftliche Sukzession, 2015, S. 509 ff.

60 Vgl. Begr. RegE, BT-Drucks. 19/29371, S. 57. 
gen und Änderungen gilt nach $\ 14$ Abs. $3, \$ 18$ Abs. 3 eWpG das Prioritätsprinzip. Auf diese Weise können widersprechende Verfügungen vermieden und die Ordnungsmäßigkeit der Registerführung gewährleistet werden. Insbesondere können die Reihenfolge und der zeitliche Ablauf von Verfügungen über elektronische Wertpapiere rechtssicher nachvollzogen werden.

Weisungen können zunächst vom Inhaber des eingetragenen Wertpapiers stammen. Die Registerführung hat ihnen nachzukommen, es sei denn, ihr ist bekannt, dass der Erklärende nicht berechtigt ist. Liegen keine Anhaltspunkte für eine mangelnde Berechtigung vor, hat die Weisung des Inhabers die Vermutung für sich, dass sie vom materiell Berechtigten stammt. ${ }^{61}$ Ist der Inhaber Nichtberechtigter, dann kann der materiell Berechtigte nach allgemeinen zivilrechtlichen Grundsätzen gegen den Inhaber einen Anspruch auf Weisungserteilung haben. Dieser Anspruch kann aus einem etwaigen rechtsgeschäftlichen oder rechtsgeschäftsähnlichen Verhältnis der beiden Beteiligten herrühren, folgt in Ermangelung eines solchen aber jedenfalls aus Eingriffskondiktion nach $\$ 812$ Abs. 1 S. 1 Alt. 2 BGB. Erlangtes Etwas ist die unrichtige Eintragung in einem Register (Registerposition), der gewisse Rechtswirkungen zukommen. ${ }^{62}$

Ferner kann die Weisung von einer Person oder Stelle herrühren, die durch Gesetz, aufgrund eines Gesetzes, durch Rechtsgeschäft, gerichtliche Entscheidung oder vollstreckbaren Verwaltungsakt hierzu berechtigt ist. Das betrifft Weisungen von gesetzlichen, rechtsgeschäftlichen und organschaftlichen Vertretern, aber auch Gerichtsurteile, die nach $\$ 894$ ZPO eine Weisung des Inhabers ersetzen. Im Fall einer Verfügungsbeschränkung nach $\$ 13$ Abs. 2 S. 1 Nr. 1, $\$ 17$ Abs. 2 S. 1 Nr. 1 eWpG hat der Inhaber über seine Weisung hinaus der registerführenden Stelle zu versichern, dass Zustimmung der durch die Beschränkung begünstigten Person vorliegt. Ist die Eintragung des einem Dritten an dem Wertpapier zustehenden Rechts nach $\$ 13$ Abs. 2 S. 1 Nr. 2, $\$ 17$ Abs. 2 S. 1 Nr. 2 eWpG betroffen, ist der Dritte weisungsbefugt. Im Interesse der materiellen Richtigkeit des Wertpapierregisters müssen sich die Weisungsberechtigten "mittels eines geeigneten Authentifizierungsinstruments" legitimieren können ( $\$ 14$ Abs.1 S. 5, $\$ 18$ Abs.1 S. 5 eWpG). Für ein auf der Blockchain geführtes Kryptowertpapierregister handelt es sich um den privaten Schlüssel (private key), der dem Berechtigten den Zugriff auf das Kryptowertpapier ermöglicht. ${ }^{63}$ Die zugehörigen Einzelheiten, die aufgrund der Technikneutralität des Entwurfs noch weitere Gestaltungen für zentrale und Kryptowertpapierregister vorsehen können, werden einer Verordnung überantwortet (vgl. \$15 Abs. 1 S. 1 Nr. 9, $\$ 23$ Abs. 1 S. 1 Nr. 9 eWpG). Soweit sich die Weisung auf Registerangaben

${ }^{61}$ Begr. RegE, BT-Drucks. 19/26925, S. 56.

62 Vgl. MüKo/Schwab, BGB (8. Aufl. 2020), $\$ 812$ Rn.12; BeckOK/Wendehorst, BGB (Stand: 1.11.2020), $\$ 812$ Rn. 133; Erman/Buck-Heeb, BGB (16. Aufl. 2020), $\$ 812$ Rn. 5; Staudinger/Lorenz, BGB (2007), \$812 Rn. 74; Schulze/Wiese, BGB (10. Aufl. 2019), \$812 Rn. 14.

63 Lebmann, BKR 2020, 431, 435. 
bezieht, die nicht ausschließlich den Inhaber betreffen, bedarf es zudem nach \14 Abs. 2, \$18 Abs. 2 eWpG der Zustimmung des Emittenten.

\section{ee) Redaktioneller Änderungsvorschlag}

Eine Detailbetrachtung der eintragungspflichtigen Inhalte zeigt, dass die Registerangaben in zentralen und Kryptowertpapierregistern sachlich komplett deckungsgleich sind. Das gilt für die obligatorischen Angaben ( $\mathbb{S} \mathbb{S} 13$ Abs. 1 Nr. 1-7, 17 Abs. 1 Nr.1-7 eWpG) und die zusätzlichen Angaben bei Einzeleintragung ( $\mathbb{S} 13$ Abs. 2, 17 Abs. $2 \mathrm{eWpG}$ ) ebenso wie für die Angaben zur registerführenden Stelle ( $\mathbb{S} 13$ Abs. 3, 17 Abs. 3 eWpG) und für Änderungen des Registerinhalts ( $\mathbb{S} 14$ Abs. 1-5, 18 Abs. 1-5 eWpG). Abweichungen des Wortlauts sind rein sprachlicher Natur. Das zeigt sich auch an den zugehörigen Passagen der Regierungsbegründung zum Kryptowertpapierregister, die über weite Strecken recht monoton auf die Erläuterungen zum zentralen Wertpapierregister verweisen. ${ }^{64}$

Vor diesem Hintergrund entspricht es dem Gebot der regelungstechnischen Effizienz und ist Zeichen einer hochentwickelten Gesetzgebungskultur, wenn Normdubletten und gesetzliche Redundanzen weitestgehend vermieden werden. Stattdessen sollten die maßgeblichen Bestimmungen entweder im Allgemeinen Teil des eWpG vor die Klammer gezogen oder einmalig für das zentrale Register festgeschrieben und hierauf für das Kryptowertpapierregister verwiesen werden. Eine solche Vereinheitlichung bewirkt nicht nur einen regelungstechnischen Gleichlauf, sondern trägt auch zu einer einheitlichen Rechtsanwendung bei.

\section{b) Niederlegung der Emissionsbedingungen}

Während die Aufnahme der wichtigsten Informationen in das Register die Zuordnung eines hinreichend bestimmten Wertpapiers zu einem Inhaber gewährleisten soll, betrifft die Niederlegung der Emissionsbedingungen bei der registerführenden Stelle die inhaltliche Ausgestaltung des Wertpapierrechts. Wenn der Regierungsentwurf davon spricht, bei der Niederlegung handele es sich „um die eigentliche (elektronische) Verkörperung des Rechts“, dann wirkt dies etwas hochgegriffen. Denn die „Verkörperung“ setzt voraus, dass sämtliche in \4 Abs. 4 eWpG genannten Tatbestandsvoraussetzungen erfüllt sind. Zudem wird man der inhaltlichen Bestimmtheit des Wertpapierrechts keinen generell höheren Wert zumessen können als der gegenständlichen Ausprägung des Bestimmtheitsgebots, ${ }^{65}$ das die Zuordnung des Wertpapierrechts zu einem Inhaber bewerkstelligt.

\footnotetext{
${ }^{64}$ Begr. RegE, BT-Drucks. 19/26925, S. 59 f.

${ }^{65} \mathrm{Zu}$ den Bestimmtheitskomponenten im Einzelnen vgl. Lieder, Die rechtsgeschäftliche Sukzession (2015), S. 302.
} 


\section{aa) Schutz der Authentizität und Integrität}

Nach der Legaldefinition des $\$ 4$ Abs. 7 eWpG ist unter dem Begriff der Emissionsbedingungen „der niedergelegte Inhalt des Rechts“ zu verstehen, „für das ein elektronisches Wertpapier eingetragen wird, einschließlich der Nebenbestimmungen." Die Niederlegung bedeutet nach der Legaldefinition des $\ 5$ Abs. 1 S. 1 eWpG, dass der Emittent vor der Eintragung „die Emissionsbedingungen bei der registerführenden Stelle als beständiges elektronisches Dokument jedermann zur beliebigen wiederholbaren unmittelbaren Kenntnisnahme zugänglich" macht. Durch die Niederlegung wird die Authentizität und Integrität des Inhalts des Wertpapiers in institutioneller Hinsicht abgesichert. ${ }^{66}$ Der redliche Rechtsverkehr soll sich darauf verlassen können, dass die bei der registerführenden Stelle zugänglichen Emissionsbedingungen tatsächlich vom Urheber des elektronischen Wertpapiers stammen und nicht nachträglich (durch den Urheber oder Dritte) verändert worden sind.

Ein weiteres Element zum Schutz der Integrität der Emissionsbedingungen ist die enumerative Aufzählung von Tatbeständen, die nach $\$ 5$ Abs. 2 S. $1 \mathrm{eWpG}$ eine Änderung bereits niedergelegter Bedingungen erlauben. Änderungen kommen danach in Betracht:

- durch Gesetz,

- aufgrund eines Gesetzes,

- aufgrund eines Rechtsgeschäfts

- aufgrund gerichtlicher Entscheidung oder

- aufgrund eines vollstreckbaren Verwaltungsakts.

Das schließt es insbesondere aus, dass die registerführende Stelle, der Emittent oder Dritte eigenmächtig und unbefugt Änderungen an den Emissionsbedingungen vornehmen. Von praktischer Bedeutung wird namentlich die Möglichkeit einer Änderung der Bedingungen kraft Rechtsgeschäfts sein, wenn eine entsprechende Regelung bereits in der originären Fassung der Emissionsbedingungen mit hinreichender Bestimmtheit vorgesehen ist, wie z. B. in Form einer Klausel zur Schuldnerersetzung. ${ }^{67}$ Im Übrigen sieht $\ 5$ Abs. 2 S. 2 eWpG vor, dass offenbare Unrichtigkeiten, wie z. B. erkennbare Schreibfehler, ohne Weiteres korrigiert werden können und nicht als Änderungen i. S. d. $\$ 5$ Abs. 2 S. 1 eWpG gelten.

Abgesehen von offenbaren Unrichtigkeiten werden Änderungen der Emissionsbedingungen nach $\$ 5$ Abs. $3 \mathrm{eWpG}$ erst durch (erneute) Niederlegung wirksam. Das Niederlegungserfordernis dient wiederum der Publizität und Beweissicherung im Interesse eines effektiven Verkehrsschutzes. Es darf allerdings nicht verkannt werden, dass die registerführende Stelle, die für die Authentizität

\footnotetext{
66 Vgl. Begr. RegE, BT-Drucks. 19/26925, S. 42 f.

67 Begr. RegE, BT-Drucks. 19/26925, S. 44.
} 
und Integrität der Emissionsbedingungen verantwortlich zeichnet, die materielle Richtigkeit und Vollständigkeit der Bedingungen und ihrer Änderungen nicht überprüft. ${ }^{68}$ In formeller Hinsicht verlangt $\ 5$ Abs. 4 eWpG weiter, dass die Änderungen in den geänderten Emissionsbedingungen nachvollziehbar sein müssen, so dass der Rechtsverkehr mit Blick auf die vorherigen Fassungen die vollständige Änderungshistorie der Bedingungen lückenlos überprüfen und im Ernstfall auch fehlerhafte Änderungen identifizieren und nachweisen kann. ${ }^{69}$

\section{bb) Niederlegung statt Eintragung}

Dass die Emissionsbedingungen niedergelegt und nicht vollständig in das Wertpapierregister eingetragen werden, hat Gründe der Praktikabilität und Zweckmäßigkeit für sich, aber keine besondere rechtliche oder rechtsdogmatische Qualität. Es ist nachvollziehbar, dass der Regierungsentwurf das Register von umfänglichen Angaben über den Inhalt der emittierten Wertpapiere freihält und die Registerangaben auf die zentralen Informationen des elektronischen Wertpapiers beschränkt. Das dient - in Parallele zu Grundbuch und Handelsregister $^{70}$ - der Übersichtlichkeit und Klarheit des Wertpapierregisters. Seine Publizitätsfunktion kann es nur erfüllen, wenn die dort enthaltenen Angaben übersichtlich und vergleichbar sind. Das entspricht zugleich der strengen Formalisierung des Registerrechts und ist mit Blick auf die Publizitätsfunktion des Wertpapierregisters ernst zu nehmen. ${ }^{71} \mathrm{Im}$ Übrigen orientieren sich die notwendigen Registerangaben am Depotauszug, wie sie sich schon im bisherigen Wertpapierhandel als angemessen erwiesen haben. ${ }^{72}$

Der Publizitäts- und Beweisfunktion entspricht es weiter, dass die Emissionsbedingungen in beständiger, elektronischer Form niedergelegt werden müssen, ohne dass aber eine qualifizierte elektronische Signatur nach $\$ 126 \mathrm{a}$ Abs. 1 BGB notwendig wäre. ${ }^{73}$ Die Verkehrsteilnehmer müssen über technische Hilfsmittel stets in der Lage sein, sich vom Inhalt der Emissionsbedingungen Kenntnis zu verschaffen, wie z.B. über einen entsprechenden Link. ${ }^{74}$ Diese Möglichkeit der Kenntnisnahme muss für interessierte Kreise bereits vor der

68 Begr. RegE, BT-Drucks. 19/26925, S. 44.

69 Vgl. Begr. RegE, BT-Drucks. 19/26925, S. 44.

70 Vgl. Heymann/Förster, HGB (3. Aufl. 2019), $\$ 8$ Rn.10; Staub/Koch, HGB (5. Aufl. 2009), \8 Rn.31; BeckOK/Müther, HGB (Stand: 15.1.2021), \8 Rn.11; Baumbach/Hopt/ Merkt, HGB (40. Aufl. 2021), $\$ 8$ Rn. 1, 5; Röhricht/Graf von Westphalen/Haas/Ries, HGB (5. Aufl. 2019), \$8 Rn. 4.

71 Vgl. (zum Handelsregister) Staub/Koch, HGB (5. Aufl. 2009), \$8 Rn. 45; Röhricht/Graf von Westphalen/Haas/Ries, HGB (5. Aufl. 2019), \8 Rn.4; Baumbach/Hopt/Merkt, HGB (40. Aufl. 2021), \8 Rn. 1; BeckOK/Müther, HGB (Stand: 15.1.2021), \8 Rn. 4.

72 Begr. RegE, BT-Drucks. 19/26925, S. 43.

73 Begr. RegE, BT-Drucks. 19/26925, S. 43.

74 Vgl. auch Segna, WM 2020, 2301, 2306. 
Eintragung des elektronischen Wertpapiers in das Register eröffnet werden, wie es heute schon in Form sog. "White Paper“ der Fall ist. ${ }^{75}$

\section{cc) Zugänglichkeit der Emissionsbedingungen}

Im Gegensatz zur Einsichtnahme in das Wertpapierregister, die nach $\$ 10$ Abs. 2 eWpG für Dritte von der Darlegung eines berechtigten Interesses abhängt, sind die Emissionsbedingungen grundsätzlich jedermann zugänglich zu machen, ohne dass ein berechtigtes Interesse gegenüber der registerführenden Stelle dargelegt werden müsste (vgl. $\ 5$ Abs. 1 S. 1 eWpG) ${ }^{76}$ Das impliziert, dass die Emissionsbedingungen auch außerhalb des Wertpapierregisters zugänglich sein müssen, wie z. B. auf der Internetseite der registerführenden Stelle. ${ }^{77}$ Allerdings kann nach $\ 5$ Abs. 1 S. 2 eWpG, der auf eine Änderungsvorschlag des Bundesrats zurückgeht, auf Veranlassung des Emittenten der Zugang zu den Emissionsbedingungen auf Grundlage einer Rechtsverordnung beschränkt werden. Diese Ausnahme bezieht sich namentlich auf Privatplatzierungen, wenn Wertpapiere an einen beschränkten Kreis von Investoren emittiert werden sollen. ${ }^{78}$ Für eine öffentliche Zugänglichkeit der Emissionsbedingungen besteht in einem solchen Fall kein Bedürfnis. Das deckt sich inhaltlich mit dem Aufsichtsrecht. ${ }^{79}$ Der Emittent kann von dieser Ausnahme Gebrauch machen, ist aber nicht dazu verpflichtet. ${ }^{80}$

\section{dd) Löschungsfrist}

Nicht unproblematisch ist die Löschungsregelung in $\ 5$ Abs. 1 S. 2 eWpG: Wird ein elektronisches Wertpapier nicht spätestens drei Monate nach der Niederlegung eingetragen, so hat die registerführende Stelle die niedergelegten Emissionsbedingungen zu löschen. Nun mag es sein, dass Emittenten sich dem aus \5 Abs. 1 S. 2 eWpG resultierenden Löschungsrisiko dadurch weitgehend entziehen können, dass sie Eintragung und Niederlegung parallel vorantreiben. Gleichwohl haben Emittenten keinen unmittelbaren Einfluss auf den eigentlichen Eintragungsprozess. Es erscheint daher schwerlich gerechtfertigt, ihnen das Risiko einer verspäteten Eintragung des elektronischen Wertpapiers aufzubürden. Insgesamt erscheint das Regelungsziel der Löschungsfrist durchaus zweifelhaft. Selbst wenn man der Regierungsbegründung darin folgen mag, dass die Niederlegung den „Anschein einer Emissionsabsicht“ begründet, re-

75 Begr. RegE, BT-Drucks. 19/26925, S. 43.

${ }^{76}$ Erläuternd Begr. RegE, BT-Drucks. 19/26925, S. 43 f.

77 Begr. RegE, BT-Drucks. 19/26925, S. 43 f.

78 Finanzausschuss, BT-Drucks. 19/29372, S. 55.

79 Dazu näher Preuße/Wöckener/Gillenkirch, Das Gesetz zur Einführung elektronischer Wertpapiere, BKR 2021, 460, 463.

80 Finanzausschuss, BT-Drucks. 19/29372, S. 55. 
sultiert daraus für den redlichen Rechtsverkehr keine gefestigte Vertrauensposition darauf, dass es tatsächlich in nächster Zeit zu einer Emission kommen wird. Davon abgesehen führt die Löschung gerade dazu, dass eine angestrebte Emission - wenn die Frist auch nur geringfügig überschritten wird - sich zunächst verzögern wird, weil die Emissionsbedingungen erneut niedergelegt werden müssen. Das alles spricht für eine ersatzlose Streichung des $₫ 5 \mathrm{Abs} .1$ S. 2 eWpG. Eine vermittelnde Lösung könnte darin bestehen, dass die Frist nach einer entsprechenden Entwurfsänderung - schon dann als gewahrt angesehen wird, wenn der Emittent alle gesetzlich notwendigen Schritte ergriffen hat und die Eintragung des elektronischen Wertpapiers ausschließlich von der registerführenden Stelle abhängig ist. Damit hätte es der Emittent selbst in der Hand, die zur Fristwahrung notwendigen Maßnahmen aus eigener Machtvollkommenheit vorzunehmen und die Löschungsfrist zu wahren.

\section{c) Bezugnabme auf Emissionsbedingungen}

Es entspricht der Publizitäts- und Beweisfunktion des elektronischen Wertpapierregisters, dass nicht nur die Zuordnung des Wertpapiers eindeutig aus dem Register hervorgehen muss, sondern auch der Inhalt des Wertpapierrechts im Interesse der Sicherheit und Leichtigkeit des Rechtsverkehrs wahrgenommen werden kann. Das erfolgt durch eine dauerhafte und eindeutige - elektronische - Verknüpfung zwischen der Registereintragung (Inhaber, Wertpapierkennnummer) und der bei der registerführenden Stelle niedergelegten Emissionsbedingungen. ${ }^{81}$ Die Regelung der technischen Details der Verlinkung ist nach $\$ 15$ Abs. 1 S. 1 Nr. $1, \$ 23$ Abs. 1 S. 1 Nr. 2 eWpG einer Rechtsverordnung vorbehalten.

\section{Feblerhafte Eintragung}

In der Natur der konstitutiven Eintragungswirkung liegt es, dass ohne Eintragung in das Wertpapierregister das elektronische Wertpapier nicht wirksam begeben ist. Der Wirksamkeit steht es entgegen, wenn entweder die Aufnahme der wesentlichen Angaben in das Register, die Niederlegung der Emissionsbedingungen ${ }^{82}$ oder die Verlinkung fehlt. Jede dieser drei Voraussetzungen ist als konstitutiver Bestandteil der Eintragung i.S.d. $\$ 4$ Abs. 4 eWpG für eine wirksame Rechtsbegründung unverzichtbar. Deshalb wird man schwerlich für ein abgestuftes Rangverhältnis zwischen Registerangaben, niedergelegten Emissionsbedingungen und der Verlinkung plädieren können.

Im Falle einer fehlerhaften Eintragung ist zu differenzieren: Handelt es sich bei dem Eintragungsmangel um eine offenbare Unrichtigkeit, wie z. B. offen-

\footnotetext{
${ }^{81}$ Begr. RegE, BT-Drucks. 19/26925, S. 41.

82 So auch Linardatos, ZBB 2020, 329, 339.
} 
sichtliche Schreibfehler oder Zahlendreher, oder bezieht sich die Unrichtigkeit auf einen unwesentlichen Nebenpunkt, wie z. B. das Datum, dann ist die Eintragung vorbehaltlich weiterer Mängel wirksam und von der registerführenden Stelle analog $\int 5$ Abs. 2 S. 2 eWpG ${ }^{83}$ zu berichtigen. ${ }^{84}$ Für alle übrigen Fehler gilt das Deckungs- oder Kongruenzprinzip. ${ }^{85}$ Danach ist die Eintragung wirksam, soweit sie im Hinblick auf ihren Inhalt und Umfang mit dem Begebungsvertrag übereinstimmt. Beziehen sich Einigung und Eintragung hingegen auf ganz unterschiedliche Forderungen, dann gelangt das elektronische Wertpapier nicht zum Entstehen. Das Wertpapierregister ist unrichtig und die registerführende Stelle verpflichtet, die Eintragung zu berichtigen.

Weichen Einigung und Eintragung quantitativ oder qualitativ nur zum Teil voneinander $\mathrm{ab}$, entsteht das elektronische Wertpapier nach dem Rechtsgedanken des $\$ 139$ BGB nur insoweit, als Einigung und Eintragung inhaltlich und umfänglich übereinstimmen. ${ }^{86}$ Es gilt die bereits den Römern bekannte Rechtsparömie, dass ein Mehr ein Weniger umfasst (da plus minus continet). ${ }^{87}$ Bedeutsam ist dies namentlich bei fehlerhafter Angabe des Nennbetrags der Inhaberschuldverschreibung. Bleibt etwa der vereinbarte hinter dem eingetragenen Nennbetrag zurück, dann ist die Begebung nur in dem vereinbarten Umfang wirksam und das Wertpapierregister ist von der registerführenden Stelle zu berichtigen. ${ }^{88}$ Übersteigt der vereinbarte den eingetragenen Nennbetrag, ist die Inhaberschuldverschreibung nur in Höhe des eingetragenen Betrags wirksam. ${ }^{89}$ Mit der Berichtigung der fehlerhaften Eintragung wird die Begebung im vereinbarten Umfang wirksam.

${ }^{83}$ Zur Berichtigung offenbarer Unrichtigkeiten der Emissionsbedingungen siehe oben II. 3. b) aa).

${ }^{84}$ Vgl. (zur fehlerhaften Eintragung der Kapitalerhöhung) MüKo/Lieder, GmbHG (3. Aufl. 2018), $₫ 57$ Rn.90; Ulmer/Habersack/Löbbe/Ulmer/Casper, GmbHG (2. Aufl. 2016), $\$ 55$ Rn. 58; Priester, Unwirksamkeit der Satzungsänderung bei Eintragungsfehlern?, BB 2002, 2613, 2615.

${ }^{85}$ Dazu allgemein Lieder, Die rechtsgeschäftliche Sukzession (2015), S. 562 f.

86 Vgl. (zum Grundstücksrecht) BGH WM 1985, 876, 878; Staudinger/Heinze, BGB (2018), \873 Rn.191, 196; MüKo/Kobler, BGB (8.Aufl. 2020), \$873 Rn.102; Erman/Artz, BGB (16. Aufl. 2020), $\$ 873$ Rn.25; Baur/Stürner, Sachenrecht (18. Aufl. 2009), $\$ 19$ Rn.31; Gergen, Wie kommt das Gewollte ins Grundbuch?, AcP 206 (2006), 624, 627.

87 Dazu ausf. Backhaus, In maiore minus inest, Eine justinianische „regula iuris“ in den klassischen Rechtsquellen - Herkunft, Anwendungsbereich und Funktion, ZRG RomA 100 (1983), 136ff.; vgl. ferner MüKo/Kobler, BGB (8. Aufl. 2020), \$873 Rn. 102.

$88 \mathrm{Vgl}$. (zur fehlerhaften Eintragung der Kapitalerhöhung) MüKo/Lieder, GmbHG (3. Aufl. 2018), $\$ 57$ Rn. 92; Scholz/Priester/Tebben, GmbHG (12. Aufl. 2020), $\$ 57$ Rn.60; Rowedder/Schmidt-Leithoff/Schnorbus, GmbHG (6. Aufl. 2017), \$57 Rn. 45; Ulmer/Habersack/Löbbe/Ulmer/Casper, GmbHG (2.Aufl. 2016), $\$ 55$ Rn. 58; aA Priester, BB 2002, 2613, 2615.

${ }^{89}$ Vgl. (zur fehlerhaften Eintragung der Kapitalerhöhung) Michalski/Heidinger/Leible/ Schmidt/Hermanns, GmbHG (3. Aufl. 2017), $\$ 57$ Rn. 66; MüKo/Lieder, GmbHG (3. Aufl. 2018), \$57 Rn. 92; aA Priester, BB 2002, 2613, 2615; vgl. weiter RGZ 85, 205, 207. 


\section{Registerfübrung}

Die Wertpapierregister können ihre Publizitäts- und Beweisfunktion nur erfüllen, wenn die registerführende Stelle gewissen Mindeststandards gerecht zu werden vermag. Zu diesem Zweck normiert $\$ 7 \mathrm{eWpG}$ allgemeine Verhaltenspflichten der registerführenden Stelle.

\section{a) Anforderungen an die Registerfübrung}

Oberste Priorität hat in diesem Kontext die Authentizität, Integrität und Vertraulichkeit der Daten, die nach $\$ 7$ Abs. 1 eWpG von der registerführenden Stelle im Rahmen ihrer Registerführung uneingeschränkt und jederzeit zu gewährleisten sind. Die registerführende Stelle muss namentlich den unbefugten Zugriff auf die Registerdaten und deren unbefugte Änderung mit technischen Mitteln unterbinden. Im Vordergrund steht dabei das Thema Datensicherheit. Hinzu kommt die Gefahr von Datenmanipulationen, insbesondere wenn das Register vom Emittenten selbst geführt wird..$^{90}$

$\mathrm{Zu}$ weit geht es indes, wenn die Regierungsbegründung davon spricht, es seien „die jeweils effizientesten technischen Standards“ zu erfüllen. ${ }^{91}$ Vielmehr wird man es als hinreichend erachten können, wenn die registerführende Stelle die anerkannten Standards beachtet, die dem neuesten Stand der Technik (state of the art) entsprechen. In diesem Sinne formulieren die Materialien auch zutreffend, dass etwa bei Kryptowertpapierregistern an den einschlägigen ISOoder DIN-Normen Maß zu nehmen sei. ${ }^{92}$ Fehlt es an solchen Standards, kann auf branchenübliche Best Practices zurückgegriffen werden.

\section{b) Materielle Richtigkeitsgewähr}

Die registerführende Stelle hat nach $\$ 7$ Abs. 2 S. 1 eWpG weiterhin sicherzustellen, dass das Register die bestehende Rechtslage zutreffend wiedergibt und Eintragungen sowie Umtragungen, d. h. Wechsel des Inhabers eines elektronischen Wertpapiers, vollständig und ordnungsgemäß erfolgen. Die Publizitäts- und Beweisfunktion des Wertpapierregisters steht und fällt mit der inhaltlichen Übereinstimmung von formeller Registerlage und (wirklicher) materieller Rechtslage. Dementsprechend muss es technisch möglich sein, dass Änderungen im Wertpapierregister nachvollziehbar ausgewiesen werden. Das gilt nicht nur für Änderungen, die sich mit Wirkung für die Zukunft ergeben, wie z.B. infolge einer Verfügung über das elektronische Wertpapier. Eine technische Lösung muss auch für Sachverhalte zur Verfügung stehen, in welchen

\footnotetext{
90 Vgl. Begr. RegE, BT-Drucks. 19/26925, S. 46 f.

91 Begr. RegE, BT-Drucks. 19/26925, S. 46; insgesamt kritisch auch Dubovitskaya, ZIP 2020, 2551, 2560.

92 Begr. RegE, BT-Drucks. 19/26925, S. 46.
} 
die Unwirksamkeitsfolge auf einen in der Vergangenheit liegenden Zeitpunkt zurückwirkt. Man denke etwa an bereits gebuchte Verfügungen, die gegen ein gesetzliches Verbot $(\$ 134$ BGB), die guten Sitten $(\$ 138$ BGB) oder eine Verfügungsbeschränkung verstoßen oder später angefochten worden sind $(\mathbb{} 142$ Abs. 1 BGB). Das wird sich auch in einem änderungsfesten Kryptowertpapierregister sachgerecht abbilden lassen, namentlich durch die gegenläufige Buchung einer zuvor erfolgten Änderung, deren Rückwirkung auf einen bestimmten Zeitpunkt fingiert wird.

Die Verpflichtung zur ordnungsgemäßen Registerführung wird nach $\$ 7$ Abs. 2 S. 2 eWpG durch eine Schadensersatzpflicht der registerführenden Stelle gegenüber dem materiell Berechtigten ${ }^{93}$ flankierend abgesichert. In rechtsdogmatischer Hinsicht handelt es sich um eine Haftung wegen vermuteten Verschuldens. Der gesetzliche Haftungstatbestand, der auf den Ersatz des dem Berechtigten entstandenen Vermögensschaden gerichtet ist, setzt keine bestehende Sonderverbindung zwischen registerführender Stelle und materiell Berechtigtem voraus. Zurückzuweisen ist daher auch die Auffassung, die von einer Anspruchsbegründung nach $\$ 280$ Abs. 1 BGB in Verbindung mit den Grundsätzen des Vertrags mit Schutzwirkung zugunsten Dritter ausgeht. ${ }^{94}$ Denn $\$ 7$ Abs. 2 eWpG begründet nicht nur eine Pflicht, deren Verletzung über $\$ 280$ Abs. 1 BGB sanktioniert werden könnte, sondern normiert einen eigenständigen Haftungstatbestand, der als lex specialis den allgemeinen Grundsätzen des bürgerlichrechtlichen Leistungsstörungsrechts vorgeht.

\section{c) Datensicherbeit}

Die registerführende Stelle zeichnet weiter für den Schutz der Datensicherheit verantwortlich. Nach Maßgabe des $\$ 7$ Abs. 3 S. 1 eWpG sind die notwendigen technischen und organisatorischen Maßnahmen zu treffen, um einen Datenverlust und eine unbefugte Datenveränderung effektiv zu verhindern. Einmal gespeicherte Daten dürfen also nicht verloren gehen, unterdrückt oder auf sonstige Weise entzogen werden. Diese Anforderungen müssen zum einen innerhalb der registerführenden Stelle durch entsprechende Compliance-Regelungen sichergestellt werden. Zum anderen muss das Registersystem auch vor Angriffen und Manipulationen von außen geschützt werden. Das läuft im Ergebnis auf die Einrichtung eines dem aktuellen Stand der Technik genügenden IT-Sicherheitssystems hinaus. ${ }^{95}$ Wird gegen diese Verpflichtungen zur Gewährleistung von

93 Unberechtigte Kritik bei Dubovitskaya, ZIP 2020, 2551, 2561, die übersieht, dass für die Frage der Schädigung des Berechtigten auf den Zeitpunkt abzustellen ist, zu welchem die registerführende Stelle ihre Pflichten konkret verletzt hat, nicht indes auf einen etwaigen späteren Zeitpunkt, zu welchem der Geschädigte seine Berechtigung etwa durch redlichen Dritterwerb bereits verloren hat.

94 So aber Sickinger/Thelen, AG 2020, 862 Rn. 48.

95 Begr. RegE, BT-Drucks. 19/26925, S. 47. 
Datensicherheit verstoßen, haftet die registerführende Stelle dem Berechtigen wiederum auf Schadensersatz. Anders aber als bei der allgemeinen Verpflichtung zur ordnungsgemäßen Registerführung handelt es sich nach $\$ 7$ Abs. 3 S. 2 eWpG um eine verschuldensunabhängige Garantiehaftung. Der registerführenden Stelle ist eine Exkulpation demnach verwehrt.

\section{Wertpapierrechtlicher Verpflichtungstatbestand}

Während die Eintragung als elektronischer Skripturakt im Zentrum der zivilrechtlichen Regelungen des eWpG steht, übt sich der Entwurf in bewusster Zurückhaltung, was das umstrittene rechtsgeschäftliche Element des wertpapierrechtlichen Verpflichtungstatbestands anbelangt. ${ }^{96}$ Die Entwurfsverfasser mögen gerade keine normativen Festlegungen für die Begebung treffen, insbesondere nicht den traditionsreichen Streit über die Wertpapierrechtstheorie entscheiden. Zu groß sind die Bedenken, die für elektronische Wertpapiere ersonnenen Regelungen könnten sich zum Meinungsstand bei verbrieften Wertpapieren in Widerspruch setzen. Und auch eine eigenständige Begebungstheorie für elektronische Wertpapiere wollen die Entwurfsverfasser vermeiden. Wenig konsequent erscheint es nach dieser Vorrede indes, wenn die Begründung im gleichen Atemzug auf eine herrschende Vertragstheorie verweist und feststellt, dass ein elektronisches Wertpapier - ebenso wie sonstige Wertpapiere - „durch Einigung zwischen dem Emittenten und dem Inhaber (Begebungsvertrag) und einem Skripturakt" entstehe. ${ }^{97}$

An dieser Stelle sollten die Entwurfsverfasser nacharbeiten. Zum einen erscheinen die Bedenken, aus einer gesetzlichen Regelung des rechtsgeschäftlichen Elements könnten Friktionen für das gesamte Wertpapierrecht entstehen, als wenig überzeugend. In Rechtsprechung ${ }^{98}$ und Schrifttum ${ }^{99}$ hat sich inzwischen die - auch als modifizierte Vertragstheorie bezeichnete - Rechtsscheintheorie durchgesetzt. Zum anderen sind keine sachlichen Gründe ersichtlich, weshalb eine moderne Kodifikation keine klare Aussage zum Erfordernis des Begebungsvertrags treffen sollte. Mit einer ausdrücklichen Regelung wäre ein Mehr an Rechtssicherheit verbunden. Umgekehrt erscheint der Meinungsstand - nicht zuletzt mit Blick auf die einschlägige BGH-Rechtsprechung ${ }^{100}$ inzwischen soweit gefestigt, dass die Praxis auch mit einer schlanken Regelung

96 Dazu und zum Folgenden Begr. RegE, BT-Drucks. 19/26925, S. 37; zustimmend Preuße/Wöckener/Gillenkirch, BKR 2020, 551, 554; Saive, ZRP 2020, 219, 220.

97 Begr. RegE, BT-Drucks. 19/26925, S. 37.

98 BGH, Urteil v. 30. 11. 1972 - II ZR 70/71 = NJW 1973, 282, 283; BGH, Urteil v. 14. 5. 2013 - XI ZR 160/12 = BKR 2013, 334 Rn. 9.

99 MüKo/Habersack, BGB (8. Aufl. 2020), Vor $\$ 793$ Rn. 26, 30f.; Staudinger/Marburger, BGB (2015), Vor $\int \mathbb{S} 793$ ff. Rn. 18; Erman/Wilhelmi, BGB (16. Aufl. 2020), $\$ 793$ Rn. 2; BeckOK/Gebrlein, BGB (57. Ed. Stand: 1.2.2021), \$793 Rn. 7.

100 Siehe nochmals Fn. 89. 
gut leben kann. Die Entwurfsverfasser sollten sich also entscheiden: Entweder lassen sie die Frage offen und verweisen damit implizit auf den hergebrachten Meinungsstand, wobei die herrschende Meinung gerade nicht der überkommenen Vertragstheorie, sondern der Rechtsschein- oder modifizierten Vertragstheorie folgt, oder sie bekennen Farbe und normieren die Grundsätze der wertpapierrechtlichen Rechtsscheintheorie.

Dies gilt ungeachtet des Umstands, dass man die Materialien auch in ihrer vorliegenden Fassung in diesem Sinne wird interpretieren müssen. ${ }^{101}$ Die Entstehung eines elektronischen Wertpapier setzt folglich neben der Eintragung in das Wertpapierregister im Grundsatz eine Einigung zwischen dem Emittenten (Schuldner) und dem ersten Wertpapiernehmer (Gläubiger) voraus. In der Emissionspraxis beinhaltet der zwischen dem Emittenten und der Emissionsbank oder dem Emissionskonsortium geschlossene Übernahmevertrag typischerweise den Begebungsvertrag. ${ }^{102}$ Behandelt man das elektronische Wertpapier nach $\$ 2$ Abs. $3 \mathrm{eWpG}$ als Sache ${ }^{103}$, dann ist es nur konsequent, den Begehungsvertrag nicht als reinen Schuldvertrag zu qualifizieren, sondern ihn in Übereinstimmung mit der Kreation von durch Urkunden verbrieften Wertpapieren ${ }^{104}$ - zugleich als dinglichen Vertrag aufzufassen. ${ }^{105}$

Schließlich ist allen Diskussionsbeiträgen eine Absage zu erteilen, die sich für eine Entstehung elektronischer Wertpapiere ohne Begebungsvertrag allein auf Grundlage der Registereintragung starkmachen. ${ }^{106}$ Das im Begebungsvertrag zum Ausdruck kommende Einigungsprinzip gehört zu den systemprägenden Grundkonstituanten des deutschen Privatrechts. ${ }^{107}$ In funktionaler Hinsicht verhindert das Einigungsprinzip zum einen, dass der Emittent sich ohne seinen Willen gegenüber einem Forderungsgläubiger verpflichtet. Zum anderen bewahrt es potenzielle Gläubiger davor, dass ihnen ein Vermögensrecht gegen oder auch nur ohne ihren Willen aufgedrängt wird. Auf einen Begebungsvertrag kann in Ermangelung tauglicher Alternativen nicht verzichtet werden. Das gilt umso mehr, wenn man dem sachenrechtlichen Grundansatz folgt, weil zur

101 So auch Döding/Wentz, WM 2020, 2312, 2313; Linardatos, ZBB 2020, 329, 340; Preuße/Wöckener/Gillenkirch, BKR 2020, 551, 554; Segna, WM 2020, 2301, 2306.

102 Baums, Recht der Unternehmensfinanzierung (2017), \$37 Rn.10; Diekmann in Habersack/Mülbert/Schlitt, Unternehmensfinanzierung am Kapitalmarkt (4. Aufl. 2019), Rn. 31.26; Müller, in: Kümpel/Mülbert/Früh/Seyfried (Hrsg.), Bank- und Kapitalmarktrecht (5. Aufl. 2019), Rn. 15.176.

103 Siehe nochmals oben II. 2. b).

$104 \mathrm{Vgl}$. Baumbach/Hefermehl/Casper, WG, ScheckG (24.Aufl. 2020), WertpapierR Rn. 33; MüKo/Habersack, BGB (8. Aufl. 2020), Vor \793 Rn. 26.

105 Segna, WM 2020, 2301, 2306; offenbar auch Kleinert/Mayer, EuZW 2020, 1059, 1060.

106 Casper, BKR 2019, 209, 214; Dubovitskaya, ZIP 2020, 2551, 2554; ebenso ablehnend zum Erfordernis einer dinglichen Einigung bei der Verfügung Lebmann, BKR 2020, 431, $436 f$. S. $231 \mathrm{ff}$

107 Speziell zum Sukzessionsrecht ausf. Lieder, Die rechtsgeschäftliche Sukzession (2015), 
(rechtsgeschäftlichen) Begründung und Übertragung dinglicher Rechte stets ein Willenselement erforderlich ist.

\section{Publizität elektronischer Wertpapiere}

Es liegt in der Konsequenz des sachenrechtlichen Ansatzes des eWpG, auch für die Ausformung des Publizitätsprinzips an sachenrechtlichen Grundsätzen Maß zu nehmen. ${ }^{108}$ Deren Grundanliegen ist es, für Offenkundigkeit der an einem Gegenstand bestehenden Verhältnisse zu sorgen. Dementsprechend muss es auch der Publizität elektronischer Wertpapiere darum gehen, die an den Papieren bestehenden, der Sinneswahrnehmung verschlossenen Rechtsverhältnisse durch den Einsatz äußerer Rechtszeichen sinnlich wahrnehmbar zu machen. ${ }^{109}$

\section{1. Überblick und Grundlagen}

Der Regierungsentwurf sieht ein überaus heterogenes System unterschiedlicher Publizitätserfordernisse vor. Neben der bereits erwähnten Transparenz der Emissionsbedingungen ${ }^{110}$ ist das Einsichtsrecht von Teilnehmern und Dritten in das Wertpapierregister nach $\$ 10$ Abs. 1 und Abs. 2 eWpG von zentraler Bedeutung. Hinzu kommt ein weitergehender Auskunftsanspruch nach $\$ 10$ Abs. 3 eWpG. Bei Einzeleintragung kann der Inhaber einen Registerauszug $(\mathbb{1 9} \mathrm{eWpG}$ ), bei Sammeleintragung eine Depotbescheinigung ( $\$ 6$ Abs. 2 DepotG) beanspruchen. Zudem hat der Emittent Eintragungen und Änderungen im Bundesanzeiger zu veröffentlichen ( $\$ 20$ Abs. 1 und Abs. 2 eWpG). Hiervon hat er die Aufsichtsbehörde in Kenntnis zu setzen, die ihrerseits nochmals die wesentlichen Informationen online veröffentlichen muss ( $\$ 20 \mathrm{Abs.} 3 \mathrm{eWpG}$ ).

Unter Hinweis auf die Besonderheiten der Blockchain-Technologie sind die weitreichenden Publizitätserfordernisse heftig kritisiert worden. ${ }^{111}$ Es gehöre zu den Charakteristika der Blockchain, dass sie ohne Registeröffentlichkeit und ohne Registerschein auskomme. Einer besonderen Publizität bedürfe es daher nicht. Die Berechtigung einer Person werde nicht durch Einsichtnahme in das Register überprüft, sondern durch einen private key nachgewiesen. Soweit es mit Blick auf Geldwäschebekämpfung, Terrorismusfinanzierung, Steuerhin-

\footnotetext{
108 Zum Publizitätsprinzip im Privatrecht allgemein siehe den Überblick bei Merkt, Unternehmenspublizität (2001), S. $17 \mathrm{ff}$.

109 Zum Publizitätsprinzip und zur Offenkundigkeit sachenrechtlicher Verhältnisse vgl. Lieder, Die rechtsgeschäftliche Sukzession (2015), S.366ff.; vgl. weiter Quantz, Besitz und Publizität im Recht der beweglichen Sachen (2005), S. 25; speziell zu elektronischen Wertpapieren Linardatos, ZBB 2020, 329, 339; Segna, Bucheffekten (2018), S. 491.

110 Siehe nochmals oben II. 3. b).

111 Dezidiert zum Folgenden Lehmann, BKR 2020, 431, 437.
} 
terziehung und anderen rechtswidrigen Machenschaften auf die Identität der Wertpapierinhaber ankomme, sollte die registerführende Stelle verpflichtet sein, die dort hinterlegten Daten an die Aufsichts- und Strafverfolgungsbehörden herauszugeben.

Diese Kritik zielt in der Sache auf die Schaffung eines Sonderrechts für Blockchain-Wertpapierregister ab. Das setzt sich allerdings zum Grundansatz des eWpG in Widerspruch, der auf eine technikneutrale Ausgestaltung der Wertpapierregister gerichtet ist. ${ }^{112}$ Es sollte gerade kein Blockchain-Wertpapier geschaffen werden, und die Verwendung der Distributed Ledger Technology sollte nicht obligatorisch sein. Plädiert man dessen ungeachtet für ein Sonderrecht für Blockchain-Wertpapierregister, wird man vielerlei Abgrenzungsfragen - nicht zuletzt in technischer Hinsicht - beantworten müssen. Darüber hinaus erweist sich die Gewährleistung von Publizität auch mit Blick auf die Nutzung der Blockchain-Technologie als unschädlich und auch nicht mit größerem Aufwand verbunden, weil die relevanten Informationen ohnehin von der registerführenden Stelle vorgehalten werden müssen. Mit Blick auf Übertragungsvorgänge und Rechtsänderungen ist eine Eintragung in das Register unabdingbar. Wenn aber die Informationen ohnehin in das Register eingetragen werden müssen, dann können sich an diese Angaben auch die für elektronischen Wertpapiere vorgesehenen Rechtsfolgen knüpfen.

\section{Berechtigung zur Einsichtnabme}

Im Gegensatz zur uneingeschränkten Zugänglichkeit der Emissionsbedingungen hat das Recht zur Einsichtnahme in das elektronische Wertpapierregister und die dort eingetragenen Registerangaben eine differenzierte Regelung erfahren.

\section{a) Teilnehmer}

Zugriff auf die Registerangaben haben nach $\$ 10$ Abs. $1 \mathrm{eWpG}$ zunächst die Teilnehmer des elektronischen Wertpapierregisters. Dieser Personenkreis umfasst namentlich Inhaber, Berechtigte, Emittenten und andere Betroffene eines im Register eingetragenen Wertpapiers. ${ }^{113}$ Sie sind zur Einsichtnahme berechtigt, ohne dass sie ein anerkennenswertes Bedürfnis nach Einsicht darlegen müssen. Dessen ungeachtet ergibt sich ihr Interesse bereits aus dem Umstand, dass sie in einer formellen und (oder) materiellen Rechtsbeziehung zum eingetragenen Wertpapier stehen. Da sie mit den Einzelheiten des Wertpapiers typischerweise vertraut sind, bestehen auch mit Blick auf berechtigte Geheimhaltungsinteressen keine Bedenken gegen einen uneingeschränkten Zugang zu

112 Zum Ganzen ausf. Begr. RegE, BT-Drucks. 19/26925, S. $27,58$.

113 Begr. RegE, BT-Drucks. 19/26925, S. 49. 
den Registerangaben. Diese Aspekte müssen auch Beachtung finden, wenn der konkrete Teilnehmerkreis eines Wertpapierregisters im Rahmen der Verordnungsermächtigung gem. $\ 15$ Abs. 1 S. 1 Nr. 5, $\$ 23$ Abs. 1 S. 1 Nr. 8 eWpG näher ausgestaltet wird.

\section{b) Dritte}

Wer nicht zum Kreis der Teilnehmer des elektronischen Wertpapierregisters zählt, kann Einsichtnahme nach $\$ 10$ Abs. 2 eWpG nur verlangen, wenn hierfür ein berechtigtes Interesse besteht. Das betrifft in erster Linie Personen, die entweder noch nicht oder nicht mehr im Register eingetragen sind, wie z. B. Erwerber oder Veräußerer vor bzw. nach Übertragung eines elektronischen Wertpapiers. ${ }^{114}$

\section{c) Redaktioneller Änderungsvorschlag}

Die differenzierende Regelung des Einsichtsrechts für Teilnehmer und Dritte erschließt sich aus dem Entwurfstext nur mit Mühe. Zweckmäßiger erscheint es, auf eine Differenzierung zu verzichten und die Einsichtnahme - in Anlehnung an $\$ 12$ Abs. $1 \mathrm{GBO}$ - unterschiedslos an ein berechtigtes Interesse zu knüpfen. Erforderlich, aber auch ausreichend wäre es dann, wenn der Antragsteller ein verständiges, durch die Sachlage gerechtfertigtes Interesse darlegt. ${ }^{115}$ Das bedeutet keine sachliche, sondern lediglich eine redaktionelle Änderung. Denn für die Teilnehmer des elektronischen Wertpapierregisters besteht in jedem Fall ein rechtliches Interesse. Für alle weiteren Personen bedarf es der Darlegung eines berechtigten Interesses bereits nach dem vorgeschlagenen $\$ 10$ Abs. 2 eWpG. Dass es überhaupt eines berechtigten Interesses bedarf, ergibt sich aus dem schutzwürdigen Geheimhaltungsinteresse der Eingetragenen, deren Grundrecht auf informationelle Selbstbestimmung (Art. 2 Abs. 1 iVm. Art. 1 Abs. 1 GG) berührt ist. ${ }^{116}$ Weder bloße Neugier noch die Verfolgung unbefugter Zwecke kann ein Einsichtsrecht begründen. ${ }^{117}$ Umgekehrt würde die Beschränkung auf ein rein rechtliches Interesse die Registerpublizität zu sehr beschränken. ${ }^{118}$ Deshalb wird man - im Anschluss an das Grundbuch-

114 Begr. RegE, BT-Drucks. 19/26925, S. 50.

115 Vgl. (zu $\$ 12$ GBO) OLG Düsseldorf, Beschl. v. 7.4.2015 - 9 AR 1/15 = BeckRS 2015, 8478 Rn. 11; Dembarter, GBO (31. Aufl. 2018), \$12 Rn. 7; Bauer/Schaub/Maaß, GBO (4. Aufl. 2018), $\$ 12$ Rn. 9; BeckOK/Wilsch, GBO (41.Ed. Stand: 1.2.2021), $\$ 12$ Rn.1.

116 Vgl. Begr. RegE, BT-Drucks. 19/26925, S. 50; (zu \$12 GBO) BVerfG, Beschl. v. 28.8.2000 - $1 \mathrm{BvR}$ 1307/91 = NJW 2001, 503, 505; KG, Beschl. v. 20.1.2004 - $1 \mathrm{~W} 294 / 03=$ RNotZ 2004, 464; BeckOK/Wilsch, GBO (Stand: 1.2.2021), \$12 Rn. 2.

117 Vgl. (zu $\$ 12$ GBO) OLG Düsseldorf, Beschl. v. 7.4.2015 - 9 AR 1/15 = BeckRS 2015, 8478 Rn.11; Demharter, GBO (31. Aufl. 2018), $\$ 12$ Rn.7; BeckOK/Wilsch, GBO (Stand: 1.2.2021), $\$ 12$ Rn.2.

118 Vgl. (zu \$12 GBO) OLG Zweibrücken, Beschl. v. 18.10.1988 - 3 W 115/88 = NJW 1989, 531; BeckOK/Wilsch, GBO (Stand: 1.2.2021), \$12 Rn. 2. 
recht $^{119}$ - auch ein tatsächliches, wirtschaftliches oder öffentliches Interesse ausreichen lassen können, soweit es nach den Umständen des konkreten Einzelfalls das berechtigte Geheimhaltungsinteresse der Eingetragenen überwiegt. Zudem ist im Fall einer Sammeleintragung zu berücksichtigen, dass der als Inhaber eingetragenen Wertpapiersammelbank kein schutzwertes Interesse zukommt, für den Rechtsverkehr nicht erkennbar zu sein. ${ }^{120}$

\section{Umfang des Einsichtsrechts}

Mit Blick auf das berechtigte Interesse der Teilnehmer des elektronischen Wertpapierregisters ist ihr Einsichtsrecht in sachlicher Hinsicht auf das sie gerade in ihrer Funktion als Inhaber, Berechtigter oder Betroffener spezifisch betreffende Wertpapier beschränkt. ${ }^{121}$ Weder können sie sich Kenntnis von allen im Register eingetragenen Wertpapieren verschaffen, noch auch nur den Gesamtbestand der auf eine Emission entfallenden Wertpapiere einsehen.

Diese Einschränkung des Umfangs des Einsichtsrechts entspricht der h. M. zum Grundbuchrecht ${ }^{122}$ und ist auch aus rechtspolitischer Perspektive überzeugend. Denn es bringt das Informationsinteresse des Einsichtsberechtigten mit dem Geheimhaltungsinteresse des Eingetragenen zu einem angemessenen, der Publizitätsfunktion entsprechenden Ausgleich. Nach dem Regelungsziel des $\$ 10 \mathrm{eWpG}$ ergibt sich das berechtigte Interesse zur Einsichtnahme aufgrund eines anerkennenswerten Bedürfnis in Bezug auf ein konkretes elektronisches Wertpapier und aufgrund der spezifischen Betroffenheit des die Einsicht Begehrenden. Dann ist es auch nur konsequent, das Einsichtsrecht auf solche Umstände zu beschränken, die zur Befriedigung des anerkennenswerten Bedürfnisses konkret erforderlich sind. Demgegenüber geht ein uneingeschränktes Einsichtsrecht weit über das schutzwürdige Informationsinteresse des Berechtigten hinaus. Zugleich ist es mit der Gefahr verbunden, dass für den Berechtigten an sich uninteressante Registerangaben aus reiner Neugier oder zu unlauteren Zwecken eingesehen werden. ${ }^{123}$ Das wiegt umso schwerer, als aus dem Register durchaus sensible Informationen der Betroffenen ersichtlich sind, die nicht ohne spezifisches Interesse preisgegeben werden dürfen. Das entspricht

119 Vgl. BayObLG, Beschl. v. 14.3.1991 - 2 Z 162/90 = BWNotZ 1991, 144; BeckOK/ Wilsch, GBO (Stand: 1.2.2021), $\$ 12$ Rn. 2.

${ }_{120}$ Begr. RegE, BT-Drucks. 19/26925, S. 50.

121 Begr. RegE, BT-Drucks. 19/26925, S. 49.

122 OLG München, Beschl. v. 11.12.2015 - 34 Wx 208/15 = NZM 2016, 268f.; OLG Karlsruhe, Beschl. v. 29.5.2013 - 11 Wx 40/13 = RNotZ 2014, 70; OLG Schleswig, Beschl. v.

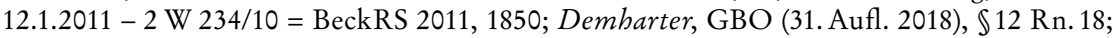
Bauer/Schaub/Maaß, GBO (4.Aufl. 2018), \$12 Rn.59; Schöner/Stöber, Grundbuchrecht (16. Aufl. 2020), Rn. 529; BeckOK/Wilsch, GBO (41.Ed. Stand: 1.2.2021), $\$ 12$ Rn.10; aA Böttcher, Grundbucheinsicht: Informationsinteresse der Presse vorrangig vor Persönlichkeitsrecht des Eigentümers, ZfIR 2011, 812, 814.

123 Vgl. (zu $\$ 12$ GBO) Bauer/Schaub/Maaß, GBO (4. Aufl. 2018), $\$ 12$ Rn. 3. 
auch der verfassungsrechtlichen Wertung des Rechts auf informationelle Selbstbestimmung.

Diese Beschränkung des Umfangs des Einsichtsrechts sollte im Gesetzestext verankert werden. Dafür würde es schon genügen, wenn den Berechtigten das Einsichtsrecht nur gewährt würde, „soweit" sie ein berechtigtes Interesse darlegen. Im Regierungsentwurf findet sich die Beschränkung bisher nur in den Erläuterungen zum Einsichtsrecht der Teilnehmer i.S.d. $\$ 10 \mathrm{Abs.} 1 \mathrm{eWpG}$, nicht hingegen in den Erläuterungen des Einsichtsrechts für berechtigte Dritte i.S.d. $\$ 10$ Abs. 2 eWpG. Das sollte idealerweise für ein vereinheitlichtes Einsichtsrecht im Gesetzestext verankert, hilfsweise in die Entwurfsbegründung aufgenommen werden.

\section{Auskunftsanspruch}

Über die aus dem Register unmittelbar ersichtlichen Angaben hinaus können ausnahmsweise noch weitere Auskünfte von der registerführenden Stelle verlangt werden, wie z. B. Informationen über die Identität und die Adresse des Inhabers eines elektronischen Wertpapiers. In Betracht kommen auch Registerangaben, die in der Vergangenheit aus dem Register abgerufen werden konnten, im Zeitpunkt des Auskunftsbegehrens aber nicht mehr aktuell und daher nicht mehr unmittelbar abrufbar sind. ${ }^{124}$ Zudem kann sich das Informationsinteresse auf weitere Kontaktdaten der Teilnehmer des elektronischen Wertpapierregisters beziehen, wie z. B. E-Mail-Adressen und Telefonnummern.

Das Auskunftsrecht besteht nach $₫ 10$ Abs. 3 S. 1 eWpG, soweit (1.) ein besonderes berechtigtes Interesse dargelegt wird, (2.) die Erteilung der Auskunft für die Erfüllung des Interesses erforderlich ist und (3.) die Interessen des Inhabers am Schutz seiner personenbezogenen Daten das Interesse des Interessenten nicht überwiegen. Ein besonderes berechtigtes Interesse besteht nach $\$ 10 \mathrm{Abs} .3$ S. 2 eWpG stets für den Inhaber eines elektronischen Wertpapiers in Bezug auf ein für ihn eingetragenes Wertpapier. Über eine besondere Berechtigung verfügt regelmäßig auch, wer personenbezogene Daten zur Rechtsdurchsetzung benötigt, die zum elektronischen Wertpapier einen spezifischen Zusammenhang aufweisen und insbesondere im Verhältnis zwischen Inhaber, Berechtigtem, Emittenten und registerführender Stelle in Betracht kommen. ${ }^{125}$

\section{Registerauszug}

Für Inhaber einzeln eingetragener Kryptowertpapiere sieht $\$ 19$ Abs. 1 eWpG vor, dass die registerführende Stelle auf Verlangen einen Registerauszug ${ }^{126}$ in Textform zur Verfügung zu stellen hat, sofern dies zur Rechtswahrnehmung,

\footnotetext{
${ }^{124}$ Dazu näher Begr. RegE, BT-Drucks. 19/26925, S. 50 f.

125 Vgl. Begr. RegE, BT-Drucks. 19/26925, S. 51.

126 Im Entwurfstext heißt es fehlerhaft: „Registerzug“.
} 
namentlich in einem Klageverfahren, erforderlich ist. Zur Begründung heißt es, dass der Nachweis über die Inhaberschaft an einem elektronischen Wertpapier oder dem Recht an einem solchen nur über den Registerinhalt geführt werden könne und Inhaltsänderungen oftmals mit einem Rechtsverlust verbunden seien. ${ }^{127}$

Ist der Inhaber ein Verbraucher i.S.d. $\$ 13$ BGB, ist die registerführende Stelle nach Maßgabe des $\$ 19$ Abs. 2 eWpG zur Ausstellung von Registerauszügen verpflichtet, wenn (1.) ein Kryptowertpapier in das Register zugunsten des Inhabers eingetragen ist, (2.) der Registerinhalt geändert wird, so dass es den Inhaber betrifft, sowie (3.) einmal im Jahr. Dem Verbraucher soll eine fortwährende Überwachung des Wertpapierregisters nicht zugemutet werden. ${ }^{128}$ Durch die automatische Information soll er stattdessen in die Lage versetzt werden, ungewollte Änderungen zu erkennen und Gegenmaßnahmen einzuleiten.

Die für beide Vorschriften angeführten Regelungsgedanken gelten indes nicht nur für Kryptowertpapiere, sondern für alle - einzeln eingetragenen elektronischen Wertpapiere, auch für Zentralregisterwertpapiere in Einzeleintragung. Für solche Wertpapiere fehlt es indes an einer vergleichbaren Regelung. Das mag darauf zurückzuführen sein, dass der Referentenentwurf die Einzeleintragung nur für Kryptowertpapiere, nicht aber auch für Zentralregisterwertpapiere vorsah. ${ }^{129}$ Nachdem der Regierungsentwurf die Einzeleintragung nun auf Zentralregisterwertpapiere erstreckt hat, ${ }^{130}$ besteht das von $\$ 19 \mathrm{eWpG}$ adressierte Informations- und Sicherungsbedürfnis für beide Wertpapierarten gleichermaßen. Der Regelungsgehalt des $\$ 19$ eWpG sollte daher auf Zentralregisterwertpapiere übertragen und als einheitliche Bestimmung im Regelungskontext der Publizitätsvorschrift des $\$ 10 \mathrm{eWp}$ normiert werden.

\section{Depotbescheinigung}

Bei sammeleingetragenen elektronischen Wertpapieren kann der Hinterleger nach $₫ 6$ Abs. 2 DepotG zur Ausübung seiner Rechte jederzeit einen in Schriftform ausgestellten Auszug über seinen Anteil am Sammelbestand verlangen. Der Verwahrer hat dabei für die materielle Richtigkeit der Depotbescheinigung einzustehen. Die mit einer entsprechenden Prüfung verbundenen Kosten können in Form eines angemessenen Aufwendungsersatzes für die tatsächlich entstandenen Aufwendungen auf den Hinterleger abgewälzt werden.

Inhaltlich wird mit der Neuregelung klargestellt, dass weder im Erkenntnis- ${ }^{131}$ noch im Vollstreckungsverfahren ${ }^{132}$ zur Rechtsausübung die physische

127 Begr. RegE, BT-Drucks. 19/26925, S. 61.

128 Dazu und zum Folgenden Begr. RegE, BT-Drucks. 19/26925, S. 61.

129 Vgl. \$8 Abs. 2 eWpG-RefE; dazu Begr. RefE, S. 49.

130 Siehe nochmals oben II. 2. a).

131 Vgl. BGH, Urteil v. 14.5.2013 - XI ZR 160/12, BKR 2013, 334.

132 Vgl. BGH, Beschl. v. 7.4.2016 - VII ZB 14/15, NZG 2016, 588. 
Auslieferung der sammelverwahrten Wertpapiere notwendig ist. ${ }^{133}$ Zur Rechtsdurchsetzung genügt die Vorlage des Auszugs aus dem Verwahrbuch nach $\$ 14$ DepotG, zu deren Ausstellung Verwahrer verpflichtet sind. Wem in der Bescheinigung der Anteil zugewiesen ist, gilt als Berechtigter. Für Beweiszwecke statuiert $\$ 6$ Abs. 2 S. 3 DepotG eine gesetzliche Fiktion. ${ }^{134}$ Der Wortlaut spricht vom Inhaber; als Inhaber eingetragen ist indes die Wertpapiersammelbank oder der Verwahrer. ${ }^{135}$ Das sollte durch eine redaktionelle Änderung korrigiert werden.

\section{Veröffentlichung im Bundesanzeiger}

Komplettiert wird das Spektrum der Publizität elektronischer Wertpapiere durch die Pflicht des Emittenten zur unverzüglichen Veröffentlichung im Bundesanzeiger der Eintragung und entsprechender Änderungen von Wertpapieren in einem Kryptowertpapierregister ( $\$ 20$ Abs. $1 \mathrm{eWpG})$. Zudem hat der Emittent die Veröffentlichung unverzüglich der Aufsichtsbehörde mitzuteilen. Anders als die Eintragung in das elektronische Wertpapierregister ${ }^{136}$ hat die Veröffentlichung rein deklaratorischen Charakter und vermittelt auch keinen öffentlichen Glauben. ${ }^{137}$

Im Interesse eines effektiven Verkehrs- und Anlegerschutzes ${ }^{138}$ sollen die wesentlichen Informationen über Kryptowertpapiere an einem zentralen Ort jedermann einfach und kostenlos zugänglich sein. Neben der Publizitätsfunktion dient die Veröffentlichungspflicht zugleich der Beweisfunktion. Durch die Veröffentlichung lassen sich insbesondere Veränderungen im Zeitablauf rechtssicher dokumentieren, auch wenn der Zugang zur Blockchain oder anderen dezentralen Aufzeichnungssystemen aus technischen oder anderen Gründen eingeschränkt sein sollte. ${ }^{139}$

$\mathrm{Zu}$ den veröffentlichungspflichtigen Informationen zählen nach $\$ 20 \mathrm{Abs.} 2$ eWp der Emittent, Informationen zum Kryptowertpapierregister, die registerführende Stelle, der wesentliche Inhalt des Rechts nebst Kennnummer und Kennzeichnung als Wertpapier, das Datum der Eintragung des Kryptowertpapiers oder einer Änderung. Die Veröffentlichung der registerführenden Stelle ist von zentraler Bedeutung, weil diese sich auch ohne Zustimmung des Inhabers nach $\$ 16$ Abs. 2 S. 3 eWpG verändern kann. Zugleich sind Anleger auf die Kenntnis der registerführenden Stelle angewiesen, weil sie die Einsichtnahme in

\footnotetext{
133 Begr. RegE, BT-Drucks. 19/26925, S. 70.

134 Begr. RegE, BT-Drucks. 19/26925, S. 70.

$135 \mathrm{Zu}$ den Formen der Inhaberschaft siehe oben II. 2.

136 Siehe nochmals oben II. 3.

137 Begr. RegE, BT-Drucks. 19/26925, S. 61.

138 Vgl. Linardatos, ZBB 2020, 329, 334.

139 Begr. RegE, BT-Drucks. 19/26925, S. 61.
} 
die Emissionsbedingungen sicherzustellen hat. ${ }^{140}$ Das ist aus rechtspolitischer Perspektive sinnvoll und verdient Beifall.

Weniger nachvollziehbar ist es hingegen, wenn die Aufsichtsbehörde nach $\$ 20$ Abs. 3 eWpG über die ihr vom Emittenten übermittelten Informationen „eine öffentliche Liste im Internet" zu führen hat. Nach den Einlassungen der Regierungsbegründung soll es sich um eine ungeprüfte Zusammenstellung der Veröffentlichungen im Bundesanzeiger handeln, ohne dass die Aufsichtsbehörde die Informationen einer formellen oder gar inhaltlichen Prüfung zu unterziehen hätte. ${ }^{141}$ Zwar erscheint es sinnvoll, dass der Aufsichtsbehörde die bezeichneten Informationen mitgeteilt werden, um im Ernstfall aufsichtsrechtliche Maßnahmen zu ergreifen. Eine weitere Veröffentlichung, wenn auch nur in der nach $\$ 20$ Abs. 3 S. 2 Nr. 1-4 eWpG reduzierten Form, bedeutet hingegen eine regulatorische Übertreibung. Mit dem Einsichtsrecht, dem Auskunftsanspruch, dem Registerauszug und der Veröffentlichung im Bundesanzeiger ist das Publizitäts- und Beweisinteresse der Inhaber elektronischer Wertpapiere hinreichend geschützt. Folglich sollte $\$ 20 \mathrm{Abs.} 3 \mathrm{eWpG}$ ersatzlos entfallen.

\section{Form- und Registerwechsel}

Dem Grundanliegen des Gesetzentwurfs zur Zulassung elektronischer Wertpapiere entspricht es, dass der Verbriefungsanspruch ausgeschlossen ist. Nach Maßgabe des $\$ 6$ Abs. 1 S. $1 \mathrm{eWpG}$ besteht kein Anspruch auf Ausreichung einzelner Wertpapierurkunden. Die dauerhafte Beibehaltung der elektronischen Form hat wirtschaftliche Gründe und Zweckmäßigkeitserwägungen für sich. Insbesondere dürfen die Beteiligten (Emittent, Inhaber, Berechtigter) darauf vertrauen, dass ein elektronisches Wertpapier dauerhaft in dieser Form existiert, übertragen und in sonstiger Weise verwendet werden kann. ${ }^{142}$ Ein Verbriefungsanspruch ist mit zusätzlichen Kosten verbunden, ohne dass dem ein individueller Nutzen des Berechtigten oder ein überindividuelles Interesse des Rechtsverkehres gegenüberstünde. Insbesondere bedarf es einer Papierurkunde nicht zur Geltendmachung des Wertpapierrechts. Aus diesem Grund kommt ein Verbriefungsanspruch des Berechtigten auch nur dann in Betracht, wenn die konkreten Emissionsbedingungen dies ausdrücklich vorsehen ( $\$ 6 \mathrm{Abs} .1$ S. $2 \mathrm{eWpG}$ ).

140 Siehe nochmals oben II. 3. b).

141 Begr. RegE, BT-Drucks. 19/26925, S. 62.

142 Dazu näher Begr. RegE, BT-Drucks. 19/26925, S. 45. 


\section{Wechsel zur Urkundenform}

Nach Maßgabe des $\$ 6$ Abs. 2 S. 1 eWpG kann der Emittent ein elektronisches Wertpapier durch ein inhaltsgleiches mittels Urkunde begebenes Wertpapier ersetzen, wenn entweder der Berechtigte zustimmt oder eine solche Ersetzung in den Emissionsbedingungen vorgesehen ist. In der Folge ist das elektronische Wertpapier aus dem Register zu löschen ( $\$ 6$ Abs. 2 S. 2 eWpG). Das urkundliche Wertpapier tritt identitätswahrend an die Stelle des elektronischen Wertpapiers, sobald die Urkunde ausgestellt und die Löschung im Wertpapierregister vollzogen ist ( $\$ 6 \mathrm{Abs.} 2 \mathrm{~S} .3 \mathrm{eWpG}$ ). Hiermit wird zugleich eine lückenlose und nachvollziehbare Übertragungskette geschaffen, welche die allgemeinen materiellen und verfahrensrechtlichen Publizitätswirkungen im Wertpapierrecht zu vermitteln vermag. In Betracht kommt danach eine Verbriefung in Form von Einzel- wie auch von sammelverwahrten Globalurkunden. ${ }^{143}$

\section{Wechsel zur Digitalform}

Für den umgekehrten Wechsel von der Urkunden- zur Digitalform hält der Entwurf eine differenzierende Regelung bereit. Im Grundsatz kann ein mittels Urkunde begebenes Wertpapier nach $\$ 6$ Abs. 4 S. 1 eWpG mit ausdrücklicher Zustimmung des Berechtigten durch ein elektronisches Wertpapier ersetzt werden. Das gilt einschränkungslos für die Eintragung mittels Urkunden begebener Wertpapiere in ein Kryptowertpapierregister. Der Berechtigte soll nicht gegen seinen Willen auf den Gebrauch technischer Instrumente verpflichtet werden, deren Verwendung aufgrund ihrer Neuartigkeit noch mit gewissen Risiken verbunden sein können. ${ }^{144}$

Für die Ersetzung durch ein Zentralregisterwertpapier ist weiter danach zu differenzieren, ob es sich um Einzelstücke oder sammelverwahrte Urkunden (Einzelurkunden oder Sammelurkunden) handelt. Während auch Einzelstücke nur mit der Zustimmung des Berechtigten umgewandelt werden können, macht $₫ 6$ Abs. 3 S. 1 eWpG für sammelverwahrte Urkunden eine Ausnahme. Ohne Zustimmung des Berechtigten kommt eine Ersetzung durch ein inhaltsgleiches Zentralregisterwertpapier in Betracht, wenn es sich (1.) um ein Wertpapier handelt, das (a) mittels Sammelurkunde begeben wurde oder (b) mittels Einzelurkunden, die in Sammelverwahrung verwahrt werden, (2.) das Zentralregisterwertpapier in einem bei einer Wertpapiersammelbank geführten zentralen Register eingetragen wird, (3.) als Inhaber eine Wertpapiersammelbank eingetragen wird und (4.) die Emissionsbedingungen eine solche Ersetzung nicht ausschließen oder von der Zustimmung des Berechtigten abhängig machen.

\footnotetext{
143 Begr. RegE, BT-Drucks. 19/26925, S. 45.

144 Begr. RegE, BT-Drucks. 19/26925, S. 46.
} 
Dass in dieser speziellen Konstellation auf die Zustimmung des Berechtigten verzichtet werden kann, beruht darauf, dass der Berechtigte auch zuvor keinen unmittelbaren Zugriff auf die sammelverwahrten Urkunden hatte. Für ihn ändert sich lediglich das Medium der Skriptur. Demgegenüber sind für ihn mit der Ersetzung durch eine Sammeleintragung weder rechtliche, wirtschaftliche noch faktische Nachteile verbunden. ${ }^{145}$ Vielmehr gelten nach $₫ 9 \mathrm{eWpG}$ für elektronische Wertpapiere in Sammeleintragung dieselben Regeln wie für sammelverwahrte Wertpapiere. Der Berechtigte kann die elektronischen Wertpapiere auch weiterhin über seine Depotbank halten, ohne dass er zu diesem Zweck ein neues Konto oder „Wallet“ einzurichten braucht. ${ }^{146}$ Auch wenn der Berechtigte nicht länger die Ausstellung und Auslieferung von Einzelurkunden verlangen kann, soweit die Schuldverschreibung nicht in Form einer Dauerglobalurkunde nach $\mathbb{9} 9$ a Abs. 3 S.2 DepotG verbrieft ist, erscheint dieser Rechtsverlust mit Blick auf die durch das eWpG angestrebte Digitalisierung des Wertpapierrechts verschmerzbar. ${ }^{147}$

\section{Zusammenfassung der wesentlichen Ergebnisse in Thesen}

1. Das Proprium elektronischer Wertpapiere liegt in der Ersetzung der urkundlichen Verbriefung durch die Eintragung des Wertpapiers in ein elektronisches Wertpapierregister.

2. Das elektronische Wertpapierregister hat eine Publizitäts- und Beweisfunktion und dient der Sicherheit und Leichtigkeit des Rechtsverkehrs. Es fungiert weniger als Transaktionsregister, sondern weist stets die aktuellen Informationen zu den eingetragenen Wertpapieren aus (Realfoliensystem).

3. Die konstitutive Wirkung der Eintragung zielt bei Wertpapieren in Einzeleintragung auf einen Gleichlauf von materieller Berechtigung und formeller Inhaberschaft ab. Das Eintragungsprinzip gewährleistet ein hohes Maß an materieller Richtigkeit und bildet die Legitimationsgrundlage für die Gutglaubens- und Vermutungswirkung des Wertpapierregisters.

4. Die Zulässigkeit von Einzel- und Sammeleintragung von Zentralregisterund Kryptowertpapieren ist rechtspolitisch überzeugend. Für die GbR als Inhaberin sollte in $\$ 8$ Abs. $1 \mathrm{Nr} .2 \mathrm{eWpG}$ noch aufgenommen werden, dass eine Eintragung in das elektronische Wertpapierregister eine Eintragung der GbR im Gesellschaftsregister voraussetzt.

5. Die Regelungen zur Eintragungspflicht von Verfügungshindernissen im Regierungsentwurf ( $\mathbb{S} \mathbb{1 3}$ Abs. 2 S. 1 Nr.1, 17 Abs. 2 S. 1 Nr. 1 eWpG-RegE)

145 Zutreffend Begr. RegE, BT-Drucks. 19/26925, S. 46; zustimmend Döding/Wentz, WM 2020, 2312, 2316; Linardatos, ZBB 2020, 329, 331; Segna, WM 2020, 2301, 2306.

146 Begr. RegE, BT-Drucks. 19/26925, S. 46.

147 So auch Segna, WM 2020, 2301, 2306. 
waren korrekturbedürftig. Zutreffend übernahm der Finanzausschuss den Vorschlag, in Bezug auf Verfügungsbeschränkungen zu differenzieren. Bestehen sie zugunsten einer bestimmten Person, sind sie eintragungspflichtig und vermögen einen redlichen Erwerb nicht zu verhindern, soweit sie nicht eingetragen sind. Demgegenüber sollte das Register von Angaben zur Geschäftsfähigkeit und absolut wirkenden Verfügungsbeschränkungen freigehalten werden.

6. Ein redaktioneller Änderungsvorschlag geht dahin, die deckungsgleichen Vorschriften über Angaben in zentralen und Kryptowertpapierregistern $(\mathbb{S} \mathbb{1 3}$, $14 \mathrm{eWpG}$ einerseits, $\mathbb{\$} \mathbb{1 7}, 18 \mathrm{eWpG}$ andererseits) einheitlich im Allgemeinen Teil des eWpG zu regeln.

7. Der eWpG sieht ein heterogenes System von Publizitätserfordernissen vor.

8. Die Unterscheidung zwischen Teilnehmern und Dritten sollte beim Einsichtsrecht aufgegeben und durch eine einheitliche Regelung ersetzt werden, die stets die Darlegung eines berechtigten Interesses verlangt. Zugleich sollte gesetzlich klargestellt werden, dass das Einsichtsrecht nur besteht, soweit das berechtigte Interesse im konkreten Einzelfall reicht.

9. Die Rechte des Inhabers auf einen Registerauszug ( $\$ 19 \mathrm{eWp}$ ) sollten für Zentralregister- und Kryptowertpapiere einheitlich ausgestaltet und im Kontext des $₫ 10 \mathrm{eWpG}$ geregelt werden.

10. Die Veröffentlichung von Eintragungen und Änderungen von Kryptowertpapieren im Bundesanzeiger ist mit Blick auf die Publizitäts- und Beweisfunktion sowie den Verkehrs- und Anlegerschutz zu begrüßen. Gleiches gilt für die Mitteilungspflicht gegenüber der Aufsichtsbehörde. Es bedarf indes keiner nochmaligen Online-Veröffentlichung der Angaben durch die Aufsichtsbehörde. 


\title{
Verfügungen über elektronische Wertpapiere
}

\author{
Sebastian Omlor
}

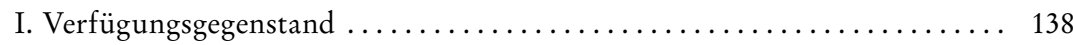

1. Ausgangspunkt für Kryptowertpapiere: allgemein-privatrechtliche

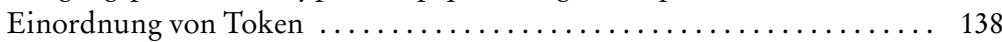

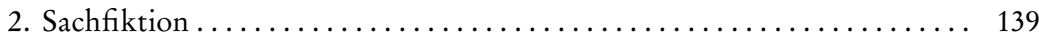

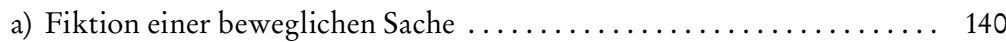

b) Fiktion einer unbeweglichen Sache $\ldots \ldots \ldots \ldots \ldots \ldots \ldots \ldots \ldots . \ldots \ldots$

c) Fiktion einer (elektronischen) Sache sui generis . ............ 141

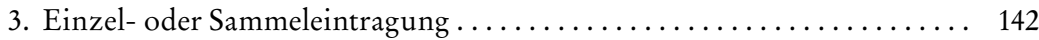

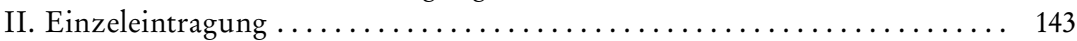

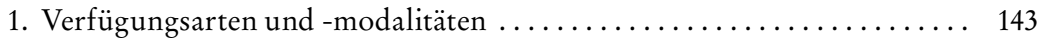

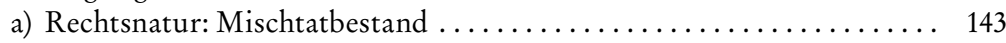

b) Verhältnis zur Abtretung der verbrieften Forderung. . . . . . . . 143

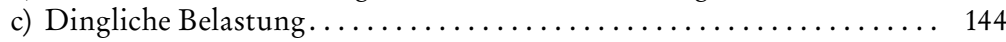

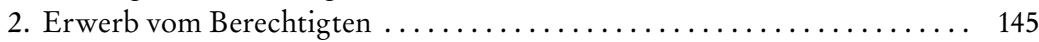

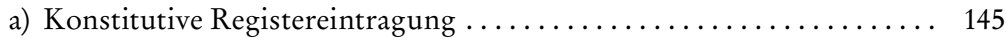

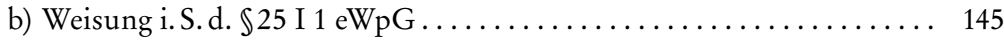

c) Berechtigung des Veräußerers ..................... 147

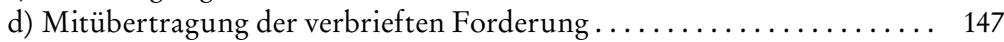

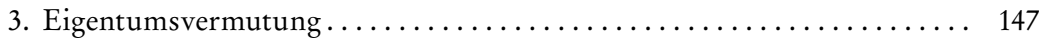

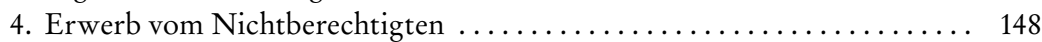

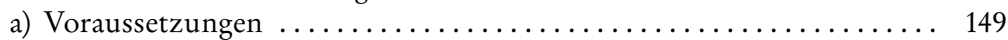

aa) Legitimation durch den Registerinhalt $\ldots \ldots \ldots \ldots \ldots \ldots \ldots \ldots . \ldots \ldots$

bb) Rechtsgeschäft ........................... 150

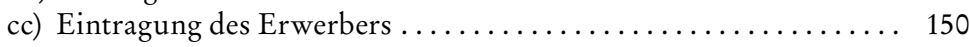

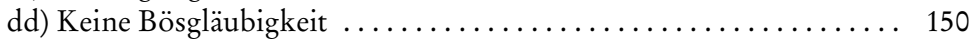

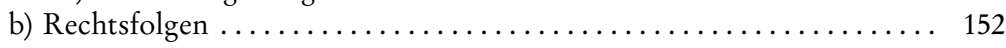

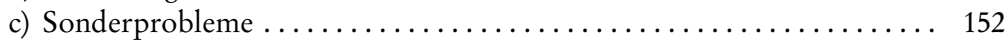

aa) Fehlende oder beschränkte Geschäftsfähigkeit .......... 152

bb) Fehlende oder beschränkte Verfügungsbefugnis . . . . . . . . 153

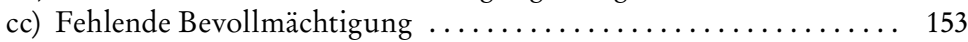

d) Rechtsverhältnis Berechtigter-Erwerber . .............. 153

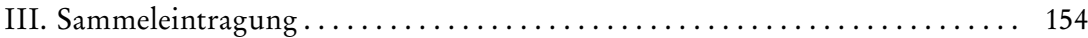

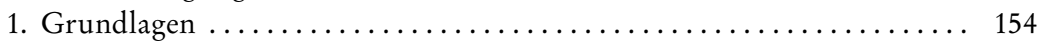

2. Übergabe von elektronischen Wertpapieren ................. 154

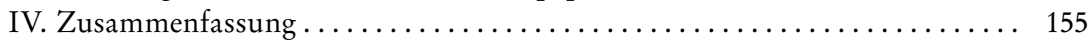


Das eWpG wendet sich in seinem vierten Abschnitt spezifisch der Verfügung über elektronische Wertpapiere in Einzeleintragung zu. Dessen Auslegung und Anwendung steht jedoch über die Sachfiktion des $\$ 2 \mathrm{Abs.} 3 \mathrm{eWpG}$ in einem Spannungsfeld mit dem BGB-Sachenrecht, das für körperliche Gegenstände verfasst wurde. Ausgeklammert werden sollten zudem elektronische Wertpapiere in Sammeleintragung, die den depotrechtlichen Regelungen unterliegen. Insgesamt leidet das eWp $\mathrm{p}$ unter dieser in nicht wenigen Aspekten diffusen $\mathrm{Hy}-$ bridcharakteristik. Der nachfolgende Beitrag versucht eine Konturenschärfung und Standortbestimmung.

\section{Verfügungsgegenstand}

Elektronische Wertpapiere treten als Zentralregisterwertpapiere oder Kryptowertpapiere auf ( $\$ 4$ Abs. 2-3 eWpG). Zur Bestimmung des Verfügungsgegenstands mit dem zugehörigen Regelungsrahmen für Verfügungen ist für Kryptowertpapiere ein Blick auf das allgemeine Privatrecht der Token zu werfen (nachfolgend, sub 1.). Für beide Arten von elektronischen Wertpapieren erweist sich die Sachfiktion von grundlegender Bedeutung, bei der klärungsbedürftig ist, ob das Mobiliar- oder Immobiliarsachenrecht oder eine Mischform in Bezug genommen werden (nachfolgend, sub 2.). Je nach Eintragungsart, d.h. Einzel- oder Sammeleintragung ( $\$ 8$ Abs. $1 \mathrm{eWpG}$ ), kommen unterschiedliche Regeln für Verfügungen zur Anwendung (nachfolgend, sub 3.).

\section{Ausgangspunkt für Kryptowertpapiere: allgemein-privatrechtliche Einordnung von Token}

Die deutsche Privatrechtsordnung ist trotz der kodifikatorischen Vorzüglichkeit des BGB weitgehend unvorbereitet für das sich immer stärker abzeichnende Blockchain-Zeitalter. ${ }^{1}$ Die einzelnen Zuordnungsgegenstände in einer dezentralen Datenbank besagter Spezifikation, für die sich auch in der deutschen Sprache der englische Begriff „Token“ etabliert hat, harren einer Einordnung in eine privatrechtliche Kategorie. ${ }^{2}$ Im Gegensatz zum anglo-amerikanischen Rechtskreis ${ }^{3}$ verhindert das starre Konzept des $\$ 90$ BGB, der zwingend eine Verkörperung erfordert, grundsätzlich eine Zuweisung der Sacheigenschaft an Token. Damit ist zugleich der Weg zu einem sachenrechtlichen Rechtsrahmen

${ }^{1}$ Rechtspolitische Forderungen zu Kryptowährungen daher bei Omlor, Blockchain-basierte Zahlungsmittel - ein Arbeitsprogramm für Gesetzgeber und Rechtswissenschaft, ZRP $2018,85 \mathrm{ff}$.

${ }^{2}$ Zum Folgenden bereits Omlor, in: ders./Link (Hrsg.), Handbuch Kryptowährungen und Token (2021), \$6 Rn. 30ff.

${ }^{3}$ Dazu Omlor, Digitales Eigentum an Blockchain-Token - rechtsvergleichende Entwicklungslinien, ZVglRWiss 119 (2020), 41, 45ff. 
versperrt. Die teilweise in der Literatur ${ }^{4}$ geforderte Annahme von partiellen Analogien trägt zwar dem Bedürfnis nach klaren und belastbaren Regeln Rechnung, gewichtet aber nicht hinreichend die starren Strukturen der lex lata. Durch die Einführung der auf elektronische Wertpapiere beschränkten Sachfiktion in $\$ 2$ Abs. 3 eWpG liegt der Umkehrschluss nochmals näher als zuvor. Die sachenrechtliche Nichteinordnung von Token jenseits des $\$ 2 \mathrm{Abs} .3 \mathrm{eWpG}$ wirkt sich mittelbar auch auf das Delikts- und Bereicherungsrecht aus. Für ein sonstiges Recht i.S.d. $\$ 823$ Abs. 1 BGB fehlt die erforderliche Eigentumsähnlichkeit, ${ }^{5}$ so dass lediglich $\$ 823$ Abs. 2 BGB i. V.m. $\$ \int 202 a, 303 a$ StGB und $\$ 826$ BGB einen fragmentarischen Schutz bieten. ${ }^{6}$ Bereicherungsrechtlich kann ein Token zwar als Etwas i.S. d. $\$ 812$ Abs. 1 BGB erlangt werden, aber mangels Zuweisungsgehalts erfahren Token, die ohne Intermediär unmittelbar durch ihren Inhaber gehalten werden, keinen Schutz durch die allgemeine Nichtleistungskondiktion in Gestalt der Eingriffskondiktion. ${ }^{7}$ Damit handelt es sich bei Token nach geltendem Privatrecht um ungeschriebene Immaterialgüterrechte ohne immaterialgüterrechtlichen Schutz. ${ }^{8}$

\section{Sachfiktion}

Für elektronische Wertpapiere greift auf Grundlage von $\$ 2$ Abs. 3 eWpG jedoch ein Sonderregime ein. Fingiert wird die Einordnung von elektronischen Wertpapieren als Sachen i.S. d. \$90 BGB. Der eWpG-Gesetzgeber erkennt mit einer solchen Fiktion an, dass es elektronischen Wertpapieren als unkörperlichen Token an sich an der Sachqualität mangelt, und dennoch das Rechts-

${ }^{4}$ Schäfer/Eckhold, in: Assmann/Schütze/Buck-Heeb (Hrsg.), Handbuch des Kapitalanlagerechts (5. Aufl. 2020), \$16a Rn. 44 (\$\$ 413, 398 ff. BGB); Koch, Die „Tokenisierung“ von Rechtspositionen als digitale Verbriefung, ZBB 2018, 359, 362 f. (\$\$ $929 \mathrm{ff}$. BGB analog); Veil, Token-Emissionen im europäischen Kapitalmarktrecht, ZHR 183 (2019), 346, 350 (\$S 873, 925 BGB analog); noch weitergehend für eine übergangsweise Gleichstellung mit körperlichen Sachen Walter, Bitcoin, Libra und sonstige Kryptowährungen aus zivilrechtlicher Sicht, NJW 2019, 3609, $3611 \mathrm{ff}$.

5 Spindler/Bille, Rechtsprobleme von Bitcoins als virtuelle Währung, WM 2014, 1357, 1359 ff.; Kaulartz, Die Blockchain-Technologie, CR 2016, 474, 478; a. A. Shmatenko/Möllenkamp, Digitale Zahlungsmittel in einer analog geprägten Rechtsordnung, MMR 2018, 495, 498; Paulus/Matzke, Smart Contracts und das BGB - Viel Lärm um nichts? -, ZfPW 2018, $431,453$.

${ }^{6}$ Im Einzelnen Omlor, in: ders./Link (Hrsg.), Handbuch Kryptowährungen und Token (2021), \$6 Rn. 67.

7 Omlor, in: ders./Link (Hrsg.), Handbuch Kryptowährungen und Token (2021), $\$ 6$ Rn. 68f.

${ }^{8}$ Engelhardt/Klein, Bitcoins - Geschäfte mit Geld, das keines ist - Technische Grundlagen und zivilrechtliche Betrachtung, MMR 2014, 355, 357 ff.; Kütük/Sorge, Bitcoin im deutschen Vollstreckungsrecht, Von der „Tulpenmanie“ zur „Bitcoinmanie“, MMR 2014, 643, 644; BeckOGK/Mössner, BGB (Stand: 01.03.2021), $\$ 90$ BGB Rn. 98 ff.; ungenau Spindler/Bille, WM 2014, 1357, 1360; Omlor, Kryptowährungen im Geldrecht, ZHR 183 (2019), 294, 308; Maute, in: Maume/Maute (Hrsg.), Rechtshandbuch Kryptowerte (2020), $\$ 4$ Rn. $20 \mathrm{ff}$. 
gewand der Sache herangezogen werden soll. Insofern unterscheidet sich die Fiktion methodisch von einer - auch unwiderleglichen - Vermutung. ${ }^{9}$ Damit müssten grundsätzlich auch die sachenrechtlichen Vorschriften einschließlich der Übertragungsregeln aus $\$ \int 929 \mathrm{ff} ., 873 \mathrm{ff}$. BGB auf elektronische Wertpapiere anzuwenden sein.

\section{a) Fiktion einer beweglichen Sache}

Problembehaftet ist bereits, ob zum Mobiliar- oder Immobiliarsachenrecht eine größere Sachnähe besteht und dementsprechend ausschließlich oder zumindest vorrangig auf die zugehörigen Regelungen zurückzugreifen ist. Das eWpG äußert sich hierzu nicht ausdrücklich. Für eine Zuordnung zu den beweglichen Sachen streitet die historische Entwicklung aus dem klassisch-analogen Wertpapierbegriff heraus, der an einem Papier und damit einer beweglichen Sache anknüpft. Rechtlich schon etwas diffuser erscheint hingegen das Zusatzargument, wonach die „Beweglichkeit“ unkörperlicher Gegenstände gegenüber den klassischen Mobilien noch weiter gesteigert sei. Das Mobiliarsachenrecht und insbesondere dessen Übertragungstatbestände greifen unmittelbar oder mittelbar auf den Besitz an der Sache zurück. Auch wenn ein einheitlicher Begriff des Besitzes abgelehnt wird, ${ }^{10}$ sind seine Bezugsobjekte unstreitig lediglich körperliche Gegenstände. ${ }^{11}$ Einen eng begrenzten Sonderfall bildet der Rechtsbesitz, der in $\iint 1029,1090$ Abs. 2 BGB verankert ist. In Durchbrechung der Beschränkungen der possessio nach klassisch römischem Recht ${ }^{12}$ werden besitzrechtliche Vorschriften auf unkörperliche Gegenstände wie Grunddienstbarkeiten erstreckt. Überdies wird der Begriff des Besitzes außerhalb des BGB nicht einheitlich verwendet. ${ }^{13}$ Vor diesem Hintergrund könnte angenommen werden, der Besitz an elektronischen Wertpapieren habe einen abweichenden Gehalt und werde durch $\$ 2$ Abs. $3 \mathrm{eWpG}$ ebenfalls fingiert.

\section{b) Fiktion einer unbeweglichen Sache}

Für eine Annäherung an das Immobiliarsachenrecht lässt sich hingegen anführen, dass auch elektronische Wertpapiere über ein Register als künstlichen Rechtsscheinträger verfügen. ${ }^{14}$ Zwar vollzieht sich die Übertragung nicht nach \873 Abs. 1 BGB durch Einigung und Eintragung; vielmehr divergiert der Tat-

\footnotetext{
${ }^{9}$ Larenz/Canaris, Methodenlehre der Rechtswissenschaft (3. Aufl. 1995), S. 245.

10 Zur Diskussion Staudinger/Gutzeit (2018), Vorbem. 34ff. zu \$S 854-872 BGB.

11 Staudinger/Gutzeit (2018), Vorbem. $43 \mathrm{f}$. zu $\$ \$ \$ 854-872$ BGB; BeckOGK/Götz, BGB (Stand: 01.07.2021), $\$ 854$ BGB Rn. $31 \mathrm{ff}$.

12 Dazu Kaser/Knütel/Lohsse, Römisches Privatrecht (22. Aufl. 2021), S. 165 f.

13 Staudinger/Gutzeit (2018), Vorbem $52 \mathrm{ff}$. zu $\$ \$ 854-872$ BGB.

14 Saive, Einführung elektronischer Wertpapiere, ZRP 2020, 219, 221; a. A. Linardatos, Elektronische Schuldverschreibungen auf den Inhaber - des Wertpapiers neue Kleider, ZBB 2020, 329, 346: „nichts mit dem Immobiliarsachenrecht zu tun“.
} 
bestand je nach Art der Verwahrung. ${ }^{15}$ Dennoch verknüpfen $\$ \$ 24,25$ Abs. 1 eWpG die elektronischen Wertpapiere in Einzeleintragung fest mit dem elektronischen Wertpapierregister, indem jegliche Verfügungen zu ihrer Wirksamkeit einen Niederschlag im Register erlangen müssen. Auch der Übereignungstatbestand des $\$ 25$ Abs. 1 Satz $1 \mathrm{eWpG}$ atmet stärker den immobiliar- als den mobiliarsachenrechtlichen Geist, da sich in ihm - wenn auch mit Modifikationen - eine Anlehnung an $\$ 873$ Abs. 1 BGB wiederfindet: Das Eigentum wird durch Umtragung im Register (auf Weisung des Berechtigten) und Einigung zwischen Erwerber und Berechtigtem übertragen. ${ }^{16}$ Einer materiell-rechtlichen Weisung bedarf es für $\$ 873$ Abs. 1 BGB zwar nicht, aber immerhin grundbuchverfahrensrechtlich existiert das formale Bewilligungserfordernis des $\mathbb{1 9}$ GBO. Auch hinsichtlich des Verkehrsschutzes durch gutgläubigen Erwerb erscheint es dogmatisch wie methodisch mühsam, die Erhöhung des elektronischen Wertpapierregisters zum Rechtsscheinträger auszublenden und Parallelen zur Besitzverschaffung nach $\$ \$ 932 \mathrm{ff}$. BGB zu ziehen. Die strukturelle Wortlautparallele von $\$ 26 \mathrm{eWpG}$ zu $\$ 892$ Abs. 1 Satz 1 BGB überschreitet die Grenze eines schlichten Zufalls. Auch ist der Gutglaubenstatbestand mit einer Eigentumsvermutung verbunden, die ebenso wie $\$ 891$ Abs. 1 BGB an den Inhalt des Registers anknüpft, nicht hingegen an den Besitz wie bei $\$ 1006$ BGB. Entsprechend erläutert die Gesetzesbegründung, die „Registerlösung [...] geht dabei partiell, etwa beim Gutglaubensschutz, über das hinaus, was die gegenwärtige Rechtslage für mittels Urkunde begebene Wertpapiere vorsieht $^{\text {"17. }}$.

\section{c) Fiktion einer (elektronischen) Sache sui generis}

Auch wenn beide Stoßrichtungen gewichtige Argumente vorweisen können, lässt sich doch eine uneingeschränkte Zuordnung zum Mobiliar- oder Immobiliarsachenrecht nicht vornehmen. Verträgt sich das elektronische Wertpapier allenfalls zähneknirschend mit den klassischen Besitzkonzeptionen mittels der dogmatisch wenig charmanten Hilfe der gesetzlichen Fiktion, hakt es immobiliarsachenrechtlich unter anderem an der privaten versus hoheitlichen Führung des Registers sowie dem fehlenden Weisungserfordernis materiell-rechtlicher Art. Entscheidend für die Einordnung und damit für die Anwendbarkeit sachenrechtlicher Vorschriften dürfte vielmehr der Inhalt der Fiktionsanordnung in $\$ 2 \mathrm{Abs} .3 \mathrm{eWpG}$ sein. Dort findet sich keine Konkretisierung, als welche Art von Sache ein elektronisches Wertpapier gilt. Stattdessen stellt die Gesetzesbegründung übergreifend auf das angestrebte Eingreifen von „dinglichen Rechts-

\footnotetext{
15 Vgl. dazu nachfolgend, sub II. und III.

${ }^{16}$ Ebenso Sickinger/Thelen, Anleihen und Genussscheine auf der Blockchain, AG 2020, 862 Rn. 22.

17 BT-Drs. 19/26925, S. 40.
} 
wirkungen“ 18 ab. Entsprechend erscheint es vorzugswürdig, in elektronischen Wertpapieren weder Mobilien noch Immobilien kraft gesetzlicher Fiktion, sondern eine neu geschaffene Kategorie von elektronischen Sachen sui generis zu erblicken. ${ }^{19}$ Die eWpG-Vorgaben fungieren dabei als leges speciales zum BGBSachenrecht. Je nach Einzelfrage kann entsprechend eine Verbindungslinie in das Mobiliar- oder Immobiliarsachenrecht führen. Zu entscheiden ist nach der Teleologie der BGB-Tatbestände im Lichte der eWpG-Vorgaben.

Kodifikationstechnisch wäre es im Sinne der Regelungsklarheit sachgerechter gewesen, keine gesetzliche Fiktion, sondern einen Verweisungstatbestand nach dem Vorbild von $\$ 90$ a Satz 3 BGB vorzusehen. Damit wird die Rechtsanwendung zwar ebenfalls nicht vor der Unsicherheit bewahrt, welche Vorschrift im Einzelfall herangezogen werden kann oder nicht. Aber immerhin wäre offengelegt, dass nicht sämtliche Vorschriften des BGB-Sachenrechts unverändert auf elektronische Wertpapiere anwendbar sein müssen, sondern lediglich entsprechende Anwendung finden können. Zudem ersparte er methodisch die Begründung, warum Teile des BGB-Sachenrechts trotz der einschränkungslosen Fiktion nicht angewendet werden können.

\section{Einzel-oder Sammeleintragung}

Elektronische Wertpapiere können in Einzel- oder Sammeleintragung gehalten werden. Ausweislich von $\$ 8$ Abs. $1 \mathrm{eWpG}$ greifen diese Alternativen sowohl bei Zentralregister- als auch bei Kryptowertpapieren. ${ }^{20}$ Die Sammeleintragung wird durch eine Wertpapiersammelbank oder einen Verwahrer verwaltet, ohne dass diese Stelle damit zum Berechtigten würde ( $\$ 9$ Abs. 2 Satz $1 \mathrm{eWpG}$ ). Die Berechtigung i.S.d. $\$ 3$ Abs. 2 eWpG liegt vielmehr bei den einzelnen Miteigentümern. Lediglich die Inhaberschaft als formelle Registerzuordnung ( $\$ 3$ Abs. 1 eWpG) kommt der Wertpapiersammelbank bzw. dem Verwahrer zu. Nach $\$ 9$ b Abs. 1 Satz 1 DepotG unterliegen elektronische Wertpapiere in Sammeleintragung weitgehend den identischen Regelungen wie sammelverwahrte Wertpapiere. Damit öffnet das eWpG je nach Eintragungsmodell die Tür zu zwei unterschiedlichen Regelungskomplexen für eine Übertragung: die Anknüpfung an den depotrechtlichen Standard zur Sammelverwahrung sowie das neue Modell zwischen eWpG-Sonderregeln und BGB-Sachenrecht infolge der Sachfiktion.

\footnotetext{
18 BT-Drs. 19/26925, S. 40.

19 Omlor, Re- statt Dematerialisierung des Sachenrechts, RDi 2021, 236 Rn. 4; vgl. einschränkend auch BT-Drs. 19/29372, S. 53.

20 Casper, in: Möslein/Omlor (Hrsg.), FinTech-Handbuch (2. Aufl. 2021), \$28 Rn. 22.
} 


\section{Einzeleintragung}

Für Verfügungen über elektronische Wertpapiere in Einzeleintragung existiert mit $\int \$$ 24-27 eWpG ein eigener Abschnitt im Gesetz. Der Rechtsnatur von elektronischen Wertpapieren entsprechend sind die Spezialregelungen systematisch mit dem BGB-Sachenrecht abzugleichen. Zu untersuchen sind die grundlegenden Verfügungsarten und -modalitäten (nachfolgend, sub 1.), der Erwerb vom Berechtigten (nachfolgend, sub 2.), die Eigentumsvermutung zugunsten des Inhabers (nachfolgend, sub 3.) und der Erwerb vom Nichtberechtigten (nachfolgend, sub 4.).

\section{Verfügungsarten und-modalitäten \\ a) Rechtsnatur: Mischtatbestand}

Der Übereignungstatbestand des $\$ 25 \mathrm{eWpG}$ ist zweigeteilt aufgebaut. Erstens bedarf es einer Umtragung im elektronischen Wertpapierregister, die auf Weisung des Berechtigten erfolgen muss. Zweitens müssen sich der veräußernde Berechtigte und der Erwerber über den Eigentumsübergang einigen. Strukturell liegt im Vergleich zum BGB-Sachenrecht ein Mischtatbestand aus $₫ 929$ Satz 1 BGB und $\ 873$ Abs. 1 BGB vor. Von $\$ 929$ Satz 1 BGB wurden Teile der Formulierung übernommen und das Übergabeerfordernis durch die Registerumtragung ersetzt. Demgegenüber ist aus $\$ 873$ Abs. 1 BGB das konstitutive Eintragungserfordernis entlehnt. Ohne eine Verlautbarung im Register ändert sich die materiell-rechtliche Zuordnung des elektronischen Wertpapiers nicht, wie $\ 25$ Abs. 1 Satz 2 eWpG klarstellt.

\section{b) Verbältnis zur Abtretung der verbrieften Forderung}

Bei elektronischen Wertpapieren in Einzeleintragung kann eine Übertragung ausschließlich nach $\$ 25 \mathrm{eWpG}$ erfolgen, so dass eine subsidiäre Heranziehung von $\iint 929 \mathrm{ff}$. BGB oder $\int 873 \mathrm{BGB}$ ausgeschlossen ist. ${ }^{21}$ Problematisch ist hingegen, ob alternativ die verbriefte Forderung nach $\$ 398$ BGB abgetreten werden könnte und nach $\$ 952$ Abs. 2 BGB i.V.m. $\$ 2$ Abs. 2 eWpG das Eigentum am elektronischen Wertpapier nachgezogen würde. ${ }^{22}$ Auszugehen ist vom übergreifenden Grundsatz der Verfügungstransparenz aus $\$ 24$ eWpG, der durch eine Forderungsabtretung außerhalb des elektronischen Wertpapierregisters

21 Casper, in: Möslein/Omlor (Hrsg.), FinTech-Handbuch (2. Aufl. 2021), \28 Rn. 46.

22 Bejahend Bialluch-von Allwörden, Zivil- und prospektrechtliche Aspekte des eWpG-E, RDi 2021, 13 Rn. 16 (zur Sammeleintragung); ablehnend J. Meier, Übertragung von elektronischen Wertpapieren nach dem eWpG-E, RDi 2021, 1 Rn. 37; Casper, in: Möslein/Omlor (Hrsg.), FinTech-Handbuch (2. Aufl. 2021), \$28 Rn.42; rechtspolitisch ebenso Linardatos, ZBB 2020, 329, 333. 
nicht ausgehöhlt werden darf. Die Gesetzesbegründung ${ }^{23}$ dürfte aber eine Abtretung nach $\$ 398 \mathrm{BGB}$ nicht generell ablehnen. Vielmehr geht sie von einer über $\ 398$ BGB hinausgehenden Wirksamkeitsanforderung an eine Abtretung aus, die aus $\mathbb{\int} 24,25$ Abs. $1 \mathrm{eWpG}$ folgt („Eintragung in das Register als weitere gesetzliche Wirksamkeitsvoraussetzung $\left.{ }^{\text {“24}}\right) \cdot{ }^{25}$ Ein solches Erfordernis kann über $\iint 398 \mathrm{f}$. BGB hinausgehend spezialgesetzlich aufgestellt werden. Selbst das vollständige Abtretungsverbot des $\$ 399$ BGB ist nicht abschließend normiert, sondern wird durch weitere Bestimmungen ergänzt. ${ }^{26}$ Daher stellt das Eintragungserfordernis nach $\$ 24 \mathrm{eWpG}$ eine neben $\$ 398$ BGB tretende Wirksamkeitsanforderung dinglicher Art dar. Zum Verfügungstatbestand für die verbriefte Forderung zählt nunmehr auch die Eintragung der nach $\ 952$ Abs. 2 BGB i. V.m. $\$ 2$ Abs. 2 eWpG simultan erfolgenden Zuordnungsänderung. In derselben juristischen Sekunde, in welcher die Eintragung erfolgt, wird die Forderungsabtretung wirksam und vollzieht sich der Eigentumsübergang am elektronischen Wertpapier.

\section{c) Dingliche Belastung}

Die Belastung von elektronischen Wertpapieren richtet sich wegen der Sachfiktion aus $\$ 2$ Abs. 3 eWpG im Grundsatz nach den BGB-Regeln. Auf eine Pfandrechtsbestellung sind daher $\$ \int 1293,1204,1205$ Abs. 1 BGB anzuwenden. ${ }^{27}$ Für den Nießbrauch an elektronischen Wertpapieren gilt grundsätzlich $\$ 1081$ BGB. Allerdings modifiziert das Prinzip der Verfügungspublizität aus $\ 24$ Nr. 1 eWpG die bürgerlich-rechtlichen Vorgaben. Die Übergabe wird durch die Registereintragung ersetzt. ${ }^{28}$ Dementsprechend erfordert eine Pfandrechtsbestellung das Vorliegen einer akzessorischen Forderung ( $\$ 2$ Abs. 3 eWpG i. V.m. $\mathbb{\int} \$ 1293,1204$ Abs. 1 BGB), die Einigung der Parteien über die Pfandrechtsbestellung ( $\$ 2$ Abs. 3 eWpG i. V.m. $\$ \int 1293,1205$ Abs. 1 Satz 1 BGB) und die Eintragung des Pfandrechts in das elektronische Wertpapierregister ( $\$ 24$ Nr. 1 eWpG). In der Folge sind der Eigentümer und der Pfandrechtsgläubiger in ihren jeweiligen Rollen in das elektronische Wertpapierregister eingetragen. Gleiches gilt für den Nießbrauch. Der von $\$ 1081$ BGB vorgesehene Mitbesitz von Eigentümer und Nießbraucher wird durch deren gleichzeitige Registereintragung in ihren jeweiligen Rollen ersetzt. ${ }^{29}$

23 BT-Drs. 19/26925, S. 66.

24 BT-Drs. 19/26925, S. 66.

25 Ebenso wohl Kleinert/Mayer, Der deutsche Weg zum elektronischen Wertpapier, EuZW 2020, 1059, 1062; Preuße/Wöckener/Gillenkirch, Der Gesetzesentwurf zur Einführung elektronischer Wertpapiere, BKR 2020, 551, 554.

26 Staudinger/Busche (2017), $\$ 399$ BGB Rn.2.

27 Casper, in: Möslein/Omlor (Hrsg.), FinTech-Handbuch (2. Aufl. 2021), $\$ 28$ Rn. 55.

28 BT-Drs. 19/26925, S. 66.

${ }^{29}$ Casper, in: Möslein/Omlor (Hrsg.), FinTech-Handbuch (2. Aufl. 2021), $\$ 28$ Rn. 56. 


\section{Erwerb vom Berechtigten}

\section{a) Konstitutive Registereintragung}

In Parallele zu $₫ 873$ Abs. $1 \mathrm{BGB}^{30}$ ist eine rechtsgeschäftliche Übertragung von elektronischen Wertpapieren in Einzeleintragung außerhalb des elektronischen Wertpapierregisters ausgeschlossen. ${ }^{31}$ Insofern besteht ein enger systematischer Zusammenhang mit der in $\$ 24 \mathrm{Nr} .1 \mathrm{eWpG}$ angeordneten Verfügungstransparenz. Ausgenommen sind naturgemäß Eigentumsübergänge kraft Gesetzes, wie sie namentlich bei der Erbfolge ( $\$ 1922$ BGB) oder bei Umwandlungsvorgängen ( $\$ 20$ Abs. $1 \mathrm{Nr} .1 \mathrm{UmwG}$ ) vorliegen. In diesen Fällen hat der neue Berechtigte eine Registerkorrektur herbeizuführen. Beispielsweise der durch Erbschein legitimierte Erbe kann eine Änderung nach $\$ 14$ Abs. 1 Satz 1 Nr. 2 lit. d, \18 Abs. 1 Satz 1 Nr. 2 lit. d eWpG herbeiführen, da ausweislich von $\$ 2353$ BGB eine gerichtliche Entscheidung zugrunde liegt. ${ }^{32}$

Bei einer Eigentumsübertragung am elektronischen Wertpapier sind der Umtragung notwendigerweise die Weisung des Berechtigten und typischerweise die dingliche Einigung zeitlich vorgelagert. Davon geht auch $\ 25$ Abs. 1 Satz 2 eWpG aus. Allerdings kann auch die Umtragung der Einigung vorausgehen. Insofern besteht eine Wertungsparallele zu $\$ 873$ Abs. 1 BGB. ${ }^{33}$ Von einer Beliebigkeit der zeitlichen Reihenfolge gehen auch $\iint 14$ Abs. 1, 18 Abs. 1 eWpG aus, da für eine Registeränderung lediglich eine vorherige Weisung des Berechtigten gefordert wird.

Die Umtragung stellt rechts- wie registertechnisch lediglich eine Unterform der Eintragung dar. Klargestellt werden soll, dass für den rechtsgeschäftlichen Erwerb nicht die Entfernung des Veräußerers als Berechtigter aus dem Register, sondern ausschließlich die Eintragung des Erwerbers als Berechtigter erforderlich ist. ${ }^{34}$ Bei einem auf Basis der Blockchain-Technologie geführten Wertpapierregister stellt sich diese Frage jedoch aus technischen Gründen nicht, da eine Austragung des Veräußerers nicht möglich ist.

\section{b) Weisung i.S.d. $\$ 25 I 1 \mathrm{eWp} G$}

Mit dem zusätzlichen Erfordernis einer Weisung durch den Berechtigten grenzt sich $\$ 25$ Abs. 1 Satz 1 eWpG von $\ 873$ Abs. 1 BGB insofern ab, als ein strukturell vergleichbares Erfordernis dort lediglich im Grundbuchverfahrensrecht

\footnotetext{
30 Ebenso Sickinger/Thelen, AG 2020, 862 Rn. 22.

31 Kleinert/Mayer, EuZW 2020, 1059, 1062; Geier, Einführung elektronischer Wertpapiere, RdF 2020, 258, 261 f.; Saive, ZRP 2020, 219, 221; Sickinger/Thelen, AG 2020, 862 Rn. 21; a. A.J. Meier, RDi 2021, 1 Rn. 39.

32 Ungenau insofern BR-Drs. 8/21, S. 62: „ausdrücklich auf das konkret eingetragene Wertpapier gerichtet".

${ }^{33}$ Zum Immobiliarsachenrecht vgl. BGH, Urt. v. 26.11.1999, Az. V ZR 432/98 = BGHZ 143, 175, 179f.; MüKo/Kohler, BGB (8. Aufl. 2020), \$873 BGB Rn. 108.

34 Casper, in: Möslein/Omlor (Hrsg.), FinTech-Handbuch (2. Aufl. 2021), $\$ 28$ Rn. 45.
} 
verankert ist. Die nach $₫ 19 \mathrm{GBO}$ erforderliche Bewilligung muss nicht notwendig vom materiell Berechtigten, sondern kann im Rahmen der Vermutung des $₫ 891$ Abs. 1 BGB auch vom formell Betroffenen erteilt werden. ${ }^{35}$ Ihre Rechtsnatur ist rein verfahrensrechtlich, nicht rechtsgeschäftlich. ${ }^{36}$ Wäre eine solche Formalisierung wie im Grundbuchrecht gewollt gewesen, hätte in $\$ 25$ Abs. 1 Satz 1 eWpG auf eine Weisung des Inhabers abgestellt werden müssen. Für ein Redaktionsversehen fehlen jegliche Anhaltspunkte. Nahe liegt zudem eine systematische Anknüpfung an das subjektive Element der Übergabe nach \$929 Satz 1 BGB. Der Besitzwechsel hat bei $\$ 929$ Satz 1 BGB auf den Willen des Veräußerers zurückführbar zu sein. ${ }^{37}$ Verbreitet wird gefordert, der Besitzübergang müsse aus Sicht beider Parteien dem Vollzug der Übereignung dienen bzw. auf diese bezogen sein. ${ }^{38}$ Ausgeschlossen werden sollen namentlich Fälle, in denen die Sache abhandengekommen ist. Eine die Übergabe zusätzlich zur dinglichen Einigung begleitende Willenserklärung wird bei $\ 929$ Satz 1 BGB hingegen nicht angenommen. ${ }^{39}$

Die Weisung des $₫ 25$ Abs. 1 Satz 1 eWpG stellt insofern eine qualifizierte Mischform des subjektiven Elements der Übergabe nach \929 Satz 1 BGB und des grundbuchrechtlichen Bewilligungsgrundsatzes dar. Mit $\$ 929$ Satz 1 BGB verbindet sie der Bezug zur Registereintragung als (untechnisches) Besitzsurrogat, mit $₫ 19 \mathrm{GBO}$ die registerführende Stelle als Erklärungsadressat. Die Weisung des $\$ 25$ Abs. 1 Satz $1 \mathrm{eWpG}$ reiht sich systematisch in das generelle Weisungserfordernis für Registeränderungen ein, das $\$ \int 14$ Abs.1, 18 Abs. 1 eWpG aufstellen. Ihrer Rechtsnatur nach könnte es sich wie bei $\$ 19$ GBO um eine verfahrensrechtliche Erklärung oder um eine Willenserklärung handeln. Für die Annahme einer Willenserklärung spricht, dass sie anders als die grundbuchrechtliche Bewilligung materiell-rechtliches Tatbestandsmerkmal des Verfügungstatbestandes ist. ${ }^{40}$ Damit muss der Berechtigte auch im Zeitpunkt der Weisungserteilung geschäftsfähig sein.

Ein Widerruf der Weisung steht dem Erklärenden bis zum Zeitpunkt ihres Zugangs ( $\$ 130 \mathrm{BGB})$ offen. ${ }^{41}$ Der Widerruf richtet sich insofern nach $\$ 130$

35 Schöner/Stöber, Grundbuchrecht (16. Aufl. 2020), Rn.100d.

36 Grundlegend BGH, Urt. v. 15.12.1972, Az. V ZR 76/71 = BGHZ 60, 46, 52; OLG München, Beschl. v. 09.05.2008, Az. 34 Wx 139/07 = RNotZ 2008, 495, 497.

37 RG, Urt. v. 10.06.1932, Az. VII 304/31 = RGZ 137, 23, 25; BGH, Urt. v. 04.10.1976, Az. VIII ZR 65/75 = BGHZ 67, 207, 208f.; eingehend zum Streitstand Staudinger/C. Heinze (2020), \$929 BGB Rn. 67 ff. m.w. N.

38 Martinek, Traditionsprinzip und Geheißerwerb, AcP 188 (1988) 573, 582f.; Palandt/ Herrler (80. Aufl. 2021), \$929 BGB Rn. 13; BeckOGK/Klinck, BGB (Stand: 01.07.2021), $\$ 929$ BGB Rn. 75 .

39 Stellvertretend BeckOGK/Klinck, BGB (Stand: 01.07.2021), \$929 BGB Rn.79 m.w. N.; zu Sonderfällen MüKo/Oechsler, BGB (8. Aufl. 2020), \$929 BGB Rn. $60 f$.

40 Im Ergebnis ebenso J. Meier, RDi 2021, 1 Rn. 40; wohl auch Casper, in: Möslein/Omlor (Hrsg.), FinTech-Handbuch (2. Aufl. 2021), \$28 Rn. 44.

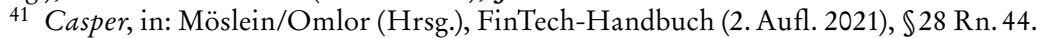


Abs. 1 Satz 2 BGB. Danach ist der Widerruf in rechtsgedanklicher Anlehnung an das allgemeine Auftrags- und Geschäftsbesorgungsrecht, das in $\$ 675 \mathrm{p}$ Abs. 1 BGB konkretisiert wurde, ${ }^{42}$ ausgeschlossen. Angesichts der Automatisierung der Registerführung würden ansonsten manuelle Eingriffe erforderlich, die zu Verzögerungen, gesteigerten Systemkosten und Haftungsrisiken führten. Ab dem Zugang der Weisung ist zumindest normativ, aber wohl auch empirisch mit einer sofortigen Umsetzung im Register zu rechnen.

\section{c) Berechtigung des Veräußerers}

Der Veräußerer muss weiterhin zur Verfügung über das elektronische Wertpapier berechtigt sein. Diese Berechtigung steht dem verfügungsbefugten Eigentümer zu. Dabei handelt es sich regelmäßig um den in das elektronische Wertpapierregister eingetragenen Inhaber, der aber nicht notwendigerweise mit dem Berechtigten personenidentisch sein muss. Zugunsten des Inhabers wirkt jedoch die widerlegliche Vermutung des $\$ 27 \mathrm{eWpG}$, die dem Erwerb vom Nichtberechtigten zugrunde liegt. Nicht ausgeschlossen ist eine Stellvertretung des Berechtigten. Nach den allgemeinen Regeln kann auch ein Vertreter ohne Vertretungsmacht handeln, dessen Erklärung nachträglich nach $\$ 177$ BGB genehmigt wird. Handelt ein Nichtberechtigter als Veräußerer, ist die Verfügung unwirksam, sofern nicht die Voraussetzungen des $\$ 26 \mathrm{eWpG}$ vorliegen.

\section{d) Mitübertragung der verbrieften Forderung}

Eine wirksame Übereignung des elektronischen Wertpapiers zieht nach $\$ 25$ Abs. 2 eWpG auch die Übertragung der verbrieften Forderung nach sich: Das Recht aus dem elektronischen Wertpapier folgt dem Recht am elektronischen Wertpapier. Damit soll die wertpapierrechtliche Transportfunktion klarstellend niedergelegt werden. ${ }^{43}$ Der Regelung kommt vor dem Hintergrund von $\$ 2$ Abs. 2 eWpG i.V.m. $\$ 793$ Abs. 1 Satz 1 BGB lediglich deklaratorische Bedeutung zu. ${ }^{44}$

\section{Eigentumsvermutung}

Eine rechtliche Verbindung zwischen Inhaber und Berechtigtem stellt die Eigentumsvermutung des $\$ 27 \mathrm{eWpG}$ her. Ähnlich wie $\$ 891$ Abs. 1 BGB und $\$ 1006 \mathrm{BGB}^{45}$ dient $\$ 27 \mathrm{eWpG}$ der Beweiserleichterung, aber zugleich als eine

42 Vgl. dazu Staudinger/Martinek/Omlor (2017), \$665 BGB Rn.7.

43 BT-Drs. 19/26925, S. 67.

44 Döding/Wentz, Der Referentenentwurf zur Einführung von elektronischen Wertpapieren und Kryptowertpapieren, WM 2020, 2312, 2316; Preuße/Wöckener/Gillenkirch, BKR 2020, 551, 554; wohl auch Kleinert/Mayer, EuZW 2020, 1059, 1063.

${ }_{45}$ Die Gesetzesbegründung nimmt nur $\$ 1006$ Abs. 1 und 2 BGB in den Blick, vgl. BRDrs. 8/21, S. 73 . 
dogmatische Stütze des gutgläubigen Erwerbs. Der Inhalt des elektronischen Wertpapierregisters und die materielle Rechtslage können auseinanderfallen. Allein die isolierte Registeränderung führt nicht zu einer Änderung der materiellen Rechtslage. Zu einer solchen Divergenz kann es namentlich bei einem gesetzlichen Eigentumsübergang (z. B. durch Erbgang nach \$1922 BGB) oder bei einer fehlerhaften Verfügung kommen, die dessen ungeachtet in das Register aufgenommen wurde. Die Vermutung des $\$ 27 \mathrm{eWpG}$ ist widerlegbar. Hierzu bedarf es eines vollen Gegenbeweises nach $\$ 292$ ZPO. Für das Eingreifen der Eigentumsvermutung kommt es nicht darauf an, ob die Registereintragung auf einer wirksamen Weisung des Berechtigten ( $\iint 14$ Abs. 1, 18 Abs. 1, 25 Abs. 1 Satz 1 eWpG) beruht. Gerade daraus resultierende Risiken will die Vermutung auffangen. Ausgenommen sind lediglich widersprüchliche Eintragungen, die beispielsweise infolge von Doppelbuchungen auftreten. ${ }^{46}$

Die Gesetzesbegründung ${ }^{47}$ sieht die Sonderregelung in $\$ 27 \mathrm{eWpG}$ als Ausgleich dafür an, dass die Sachfiktion den für $\$ 1006$ Abs. 1 und 2 BGB notwendigen Besitz nicht ersetzen könne. Diese Annahme ist zutreffend, führt aber stattdessen näher an die Vermutung des $₫ 891$ Abs. 1 BGB heran. ${ }^{48}$ Ähnlich wie der Buchbesitzer agiert der Inhaber ( $\$ 3$ Abs. $1 \mathrm{eWpG})$ als „Registerbesitzer“. Die Vermutung des $\$ 27 \mathrm{eWpG}$ reicht jedoch deutlich weniger weit als $\$ 891 \mathrm{BGB}$. Es fehlt an der negativen Vermutung des $\$ 891$ Abs. 2 BGB, zudem wird nur die Eigentümerstellung und nicht eine sonstige Rechtsinhaberschaft vermutet.

Neben $\$ 27$ eWpG findet $\$ 1006$ BGB keine - auch keine analoge - Anwendung. ${ }^{49}$ Der exklusive Zugriff auf den privaten Schlüssel zu Kryptowertpapieren in Gestalt von Token weist nur entfernt Ähnlichkeiten zur durch den Besitz vermittelten tatsächlichen Sachherrschaft auf, da es sich lediglich um ein $\mathrm{Zu}$ gangsinstrument handelt. ${ }^{50}$ Vor allem bildet $\$ 27 \mathrm{eWp}$ nach der gesetzgeberischen Konzeption eine abschließende Regelung. Die Gesetzesbegründung ${ }^{51}$ geht ausdrücklich von einer Nichtanwendbarkeit von $\$ 1006$ BGB aus. Ziel ist es, das elektronische Wertpapierregister zur exklusiven Publizitätsquelle für elektronische Wertpapiere zu erheben.

\section{Erwerb vom Nichtberechtigten}

Der Verkehrsschutz durch gutgläubigen Erwerb, der bei nicht-elektronischen Wertpapieren den klassisch-sachenrechtlichen Regeln folgt, wird mit $\$ 26 \mathrm{eWpG}$ für elektronische Wertpapiere auf eine neue Grundlage gestellt. Der Rechtsver-

46 Vgl. zum Grundbuch OLG Naumburg, Beschl. v. 25.11.2002, Az.: 1 Ss (B) 429/02 = ZfSch 2002, 275; MüKo/Kobler, BGB, (8. Aufl. 2020), \$891 BGB Rn.3.

47 BT-Drs. 19/26925, S. 67.

48 Ähnlich zurückhaltend auch Linardatos, ZBB 2020, 329, 337.

49 A. A. Kleinert/Mayer, EuZW 2020, 1059, 1063.

${ }^{50} \mathrm{Zu}$ Recht zurückhaltend auch Linardatos, ZBB 2020, 329, 333.

${ }^{51}$ BT-Drs. 19/26925, S. 67. 
kehr in der Person des Erwerbers darf auf den Inhalt des elektronischen Wertpapierregisters vertrauen. In der Folge wird ein Erwerb vom Nichtberechtigten möglich, sofern letzterer als Inhaber des übertragenen elektronischen Wertpapiers im Register verzeichnet ist.

\section{a) Voraussetzungen}

\section{a) Legitimation durch den Registerinbalt}

Das elektronische Wertpapierregister wird zum exklusiven Rechtsscheinträger für den gutgläubigen Erwerb von elektronischen Wertpapieren erhoben. Damit koppelt sich das eWpG dogmatisch weitgehend von $\$ \$ 932 \mathrm{ff}$. BGB ab. Die Registereintragung als neuartiges Übergabesurrogat einzuordnen, um die traditionelle Anknüpfung an das Mobiliarsachenrecht fortzuschreiben, wird der gesetzlichen Konzeption nicht hinreichend gerecht. Vielmehr dominiert die strukturelle Anknüpfung an das Grundbuch als künstlichen Rechtsscheinträger. Eine Modifikation erfährt die Anlehnung an $\$ 892$ Abs. 1 Satz 1 BGB vor allem durch die Erweiterung der Bösgläubigkeit, die \932 Abs. 2 BGB entnommen wurde. Die Formulierung, der Registerinhalt sei „vollständig und richtig“ und zudem gelte der „Inhaber als Berechtigter“, weist wiederholenden Charakter auf. Wenn der Registerinhalt als richtig gilt, ist damit bereits auch die Berechtigung des Inhabers umfasst. Insofern kommt diesem Zusatz lediglich eine klarstellende Funktion zu.

Der Inhalt des elektronischen Wertpapierregisters muss objektiv eine unzutreffende Aussage über die Rechtslage treffen, als der Inhaber in Wahrheit nicht der Berechtigte ist. Vom öffentlichen Glauben umfasst sind nur gesetzlich zulässige Angaben, wie sie in $\$ 13$ Abs. 1 und 2, $\$ 17$ Abs. 1 und $2 \mathrm{eWpG}$ abschließend aufgeführt sind. ${ }^{52}$ Im Fokus stehen dabei die Angaben nach $\$ 13$ Abs. 1 Nr.6, Abs. 2, $\mathbb{1 7}$ Abs. 1 Nr.6, Abs. 2 eWpG zum Inhaber, zu Verfügungshindernissen und zu Rechten Dritter. Insofern überbrückt der öffentliche Glaube des elektronischen Wertpapierregisters lediglich die Divergenz zwischen dem Rechtsschein des Registers und der wahren Rechtslage.

Zeitlich kommt es für die Legitimation des Veräußerers durch den mit öffentlichem Glauben versehenen Registerinhalt auf die Vollendung des Rechtserwerbs an. Regelmäßig wird der Rechtserwerb mit Eintragung in das elektronische Wertpapierregister vollendet, jedoch kann die Einigung der Eintragung auch nachfolgen. Wurde die Unrichtigkeit des Registers zwischen Einigung oder Weisungserteilung und Eintragung behoben, scheidet ein Rückgriff auf $\$ 26 \mathrm{eWpG}$ aus. ${ }^{53}$

\footnotetext{
52 BT-Drs. 19/26925, S. 67.

${ }^{53}$ Vgl. zur parallelen Rechtslage im Immobiliarsachenrecht BeckOGK/Eckert, BGB (Stand: 01.08.2021), \$892 BGB Rn.7.
} 


\section{bb) Rechtsgeschäft}

Ein gutgläubiger Erwerb von elektronischen Wertpapieren nach $\$ 26 \mathrm{eWpG}$ kann nur bei rechtsgeschäftlichen Übertragungsvorgängen erfolgen. Uneingeschränkt gilt das allgemeine Erfordernis der Verkehrsschutzdogmatik, wonach es für einen Erwerb vom Nichtberechtigten eines Rechtsgeschäfts im Sinne eines Verkehrsgeschäfts bedarf. ${ }^{54}$ Die Formulierung von $\$ 26 \mathrm{eWpG}$ ist insofern teilweise missglückt. Ein Erwerb „aufgrund eines Rechtsgeschäfts“ lässt sich im Lichte des Trennungs- und Abstraktionsprinzips auch auf das schuldrechtliche Verpflichtungsgeschäft beziehen. Dogmatisch präziser wäre es, in Parallele zu $\$ 892$ Abs. 1 Satz 1 BGB von einem Erwerb „durch Rechtsgeschäft“ zu sprechen. Ausgeschlossen aus dem Anwendungsbereich sind damit nicht nur Tatbestände des gesetzlichen Eigentumsübergangs (z. B. $\$ \$ 1415$ f., 1922 BGB, \$20 Abs. 1 Satz 1 Nr. 1 UmwG) und ein Erwerb in der Zwangsvollstreckung, sondern auch Veräußerungen mit einer rechtlichen oder wirtschaftlichen Personenidentität von Veräußerer und Erwerber. ${ }^{55}$

\section{cc) Eintragung des Erwerbers}

Ohne Eintragung des Erwerbers in das elektronische Wertpapierregister ist ein gutgläubiger Erwerb ausgeschlossen. Damit verweist der Wortlaut von $\$ 26$ eWpG klarstellend auf die allgemeinen Anforderungen an eine Verfügungswirksamkeit aus $\$ \mathbb{2 4}, 25 \mathrm{Abs} .1 \mathrm{eWpG}$, zu denen vor allem die konstitutive Registereintragung gehört. Deutlich wird die Funktion von Gutglaubenstatbeständen, die lediglich die fehlende Berechtigung des Veräußerers überwinden, aber nicht von den übrigen Erwerbsvoraussetzungen befreien.

\section{dd) Keine Bösgläubigkeit}

Den subjektiven Ausschlusstatbestand für einen gutgläubigen Erwerb bildet die Bösgläubigkeit des Erwerbers im maßgeblichen Zeitpunkt. In Anlehnung an $\$ 932$ Abs. 2 BGB und $\$ 16$ Abs. 3 Satz 3 GmbHG fehlt es nicht nur bei positiver Kenntnis, sondern auch bei grob fahrlässiger Unkenntnis an der Gutgläubigkeit. Anzulegen ist ein durchschnittlicher Maßstab im Sinne eines objektivierten Fahrlässigkeitsbegriffs. ${ }^{56}$ Entsprechend kann den Erwerber im Einzelfall eine Nachforschungsobliegenheit treffen, um sich nicht dem Vorwurf der gro-

${ }^{54}$ Allgemein dazu BGH, Urt. v. 29.06.2007, Az. V ZR 5/07 = BGHZ 173, 71 Rn. 22; Omlor, Verkehrsschutz im Kapitalgesellschaftsrecht (2010), S. $389 \mathrm{ff}$, jeweils m. w. N.

$55 \mathrm{Zu}$ weiteren Fällen vgl. BGH, Urt. v. 29.06.2007, Az. V ZR 5/07 = BGHZ 173, 71 Rn. 22; MüKo/Kobler, BGB (8. Aufl. 2020), $\$ 892$ BGB Rn.33; Staudinger/Picker (2019), $\$ 892$ BGB Rn. $96 \mathrm{ff}$. m. w. N.

${ }^{56}$ Vgl. zu $₫ 932$ BGB stellvertretend Staudinger/C. Heinze (2020), $\$ 932$ BGB Rn. 49 
ben Fahrlässigkeit auszusetzen. ${ }^{57}$ Der Berechtigte hat nachzuweisen, dass der Erwerber bösgläubig war, um einen gutgläubigen Erwerb auszuschließen; ihn trifft die Darlegungs- und Beweislast („es sei denn“). Die Gutgläubigkeit wird bis zum Beweis des Gegenteils vermutet.

Ebenso wie $\$ 892 \mathrm{BGB}^{58}$ setzt $\$ 26 \mathrm{eWpG}$ nicht voraus, dass der Erwerber das elektronische Wertpapierregister eingesehen hat oder ob ihm dessen Inhalt bekannt ist. ${ }^{59}$ Eines konkreten Vertrauens auf den Registerinhalt bedarf es nicht, ${ }^{60}$ so dass der unrichtige Registerinhalt nicht kausal für den Erwerbsentschluss geworden sein muss. Vielmehr genügt ein abstraktes Vertrauen des Erwerbers.

In zeitlicher Hinsicht stellt $\$ 26 \mathrm{eWpG}$ auf den Zeitpunkt der Eintragung für das Vorliegen von Bösgläubigkeit ab. Insofern liegt eine Abweichung sowohl zum Mobiliar- als auch Immobiliarsachenrecht vor. Beim gutgläubigen Erwerb von beweglichen Sachen nach $₫ 932$ BGB ist die Vornahme des letzten Erwerbsakts maßgeblich. ${ }^{61}$ Die Registereintragung stellt aber nicht notwendigerweise den letzten Erwerbsakt bei elektronischen Wertpapieren dar, da die dingliche Einigung ihr auch nachfolgen kann. Bei Grundstücksrechten, die in das Grundbuch einzutragen sind, wird ebenfalls nicht auf die Grundbucheintragung abgestellt, sondern auf den Zeitpunkt der Stellung des Eintragungsantrags ( $\$ 892$ Abs. 2 Halbsatz 1 BGB). Auf diese Weise soll der Erwerber vor Risiken infolge der Dauer des Eintragungsverfahrens geschützt werden. ${ }^{62} \$ 26$ eWpG dürfte der Gedanke zugrunde liegen, dass angesichts der unterschiedlichen Ausgestaltung von Grundbuch und Register mit solchen Verzögerungen beim elektronischen Wertpapierregister nicht zu rechnen ist. Allerdings lassen sie sich schon aus technischen Gründen nicht vollständig ausschließen. Dessen war sich der Gesetzgeber ausweislich der dazu schweigenden Gesetzesbegründung ${ }^{63}$ augenscheinlich nicht bewusst. Daher erscheint es geboten, in teleologischer Auslegung bereits auf die Erteilung der Weisung ( $\int \mathbb{S} 14$ Abs. 1, 18 Abs. 1, 25 Abs. 1 Satz 1 eWpG) statt auf die nachfolgende Eintragung abzustellen. Praktisch dürfte die Zeitspanne zwischen Weisungserteilung und Registereintragung regelmäßig sehr kurz sein, so dass diese Vorverlagerung nur selten zum Tragen kommen wird. m. w. N.

57 Vgl. zu $\$ 932$ BGB im Überblick Staudinger/C. Heinze (2020), 932 BGB Rn. 55 ff.

58 Dazu stellvertretend Staudinger/Picker (2019), $\$ 892$ BGB Rn.7f. m.w. N.

59 BT-Drs. 19/26925, S. 67.

60 Linardatos, ZBB 2020, 329, 342.

61 Statt aller BGH, Urt. v. 21.05.1953, Az. IV ZR 192/52 = BGHZ 10, 69, 73; Staudinger/C. Heinze (2020), $\$ 932$ BGB Rn. 92, jeweils m.w. N.

62 RG, Urt. v. 20.09.1933, Az. V 106/33 = RGZ 141, 379, 383.

63 BT-Drs. 19/26925, S. 67. 
b) Rechtsfolgen

Der gutgläubige Erwerber erhält nach $\ 26 \mathrm{eWpG}$ sein Recht so, als sei das elektronische Wertpapierregister richtig und vollständig gewesen. Über nicht in das Register eintragungsfähige Erwerbshindernisse hilft $\$ 26 \mathrm{eWpG}$ hingegen nicht hinweg. Der Eigentumserwerb nach $\$ 26 \mathrm{eWpG}$ ist rechtlich mit einem solchen nach $\$ 25 \mathrm{eWpG}$ vom Berechtigten gleichwertig. Es entsteht kein Recht minderer Art. Auch ein gutgläubig lastenfreier Erwerb ist möglich, sofern die Belastung nicht im elektronischen Wertpapierregister eingetragen war. Rechte Dritter wie insbesondere Pfandrechte sind nach $₫ 13$ Abs. 2 Satz 1 Nr. 2, $\$ 17$ Abs. 2 Satz 1 Nr. 2 eWpG eintragungsfähig. Zwar ist die Eintragung bei einem rechtsgeschäftlichen Erwerb nach $\$ 24 \mathrm{eWpG}$ konstitutiv, allerdings können Belastungen auch auf andere Weise - z. B. durch gesetzlichen Erwerb - erlangt werden.

\section{c) Sonderprobleme}

\section{aa) Feblende oder beschränkte Geschäftsfähigkeit}

In Abweichung zum Referentenentwurf ${ }^{64}$ schützt $\$ 26 \mathrm{eWpG}$ nicht mehr den guten Glauben an die unbeschränkte Geschäftsfähigkeit des Veräußerers. ${ }^{65} \mathrm{Da}$ bei handelt es sich um eine bewusste Entscheidung des Gesetzgebers. ${ }^{66}$ Damit wahrt das eWp zwar den systematischen Wertungseinklang mit der BGBRechtsgeschäftslehre, wird aber den technischen Besonderheiten der DLT nicht gerecht. ${ }^{67}$ Konzeptionell beruht namentlich die Blockchain-Technologie auf einer Unveränderlichkeit der Eintragungen und einem Vertrauen aller Teilnehmer in die technische Sicherheit des Systems statt in die Person eines anderen Teilnehmers. Zumindest für Kryptowertpapiere wäre es daher vorzugswürdig gewesen, den gebotenen Schutz von nicht oder nur beschränkt Geschäftsfähigen auf andere Weise als durch eine Unwirksamkeit nach den allgemeinen Regeln $(\$ \$ 104 \mathrm{ff} . \mathrm{BGB})$ vorzusehen.

Der gute Glaube an die unbeschränkte Geschäftsfähigkeit des Veräußerers wird auch nicht deshalb geschützt, weil Beschränkungen der Geschäftsfähigkeit nach $\$ 13$ Abs. 2 Satz 1 Nr. $1, \$ 17$ Abs. 2 Satz 1 Nr. 1 eWpG in das elektronische Wertpapierregister eingetragen werden können. Diesen Eintragungen kommt lediglich eine deklaratorische Wirkung zu. ${ }^{68}$ Die Beschränkung der Geschäfts-

${ }^{64}$ Dazu noch Sickinger/Thelen, AG 2020, 862 Rn.37f.; Kleinert/Mayer, EuZW 2020, 1059, 1063; Lehmann, Zeitenwende im Wertpapierrecht, BKR 2020, 431, 437; Linardatos, ZBB 2020, 329, $344 \mathrm{f}$.

${ }^{65}$ Casper, in: Möslein/Omlor (Hrsg.), FinTech-Handbuch (2. Aufl. 2021), $\$ 28$ Rn. 51.

66 BT-Drs. 19/26925, S. 67.

${ }^{67}$ Im Ergebnis ebenfalls kritisch zum Wegfall J. Meier, RDi 2021, 1 Rn. 53; vgl. zur unzureichenden Berücksichtigung von Blockchain-Spezifika Lehmann, BKR 2020, 431, 437.

68 BT-Drs. 19/26925, S. 57. 
fähigkeit bestehen kraft Gesetzes und sind nicht von einer Registereintragung abhängig. Entsprechend kann der Erwerber bei einer unterlassenen Eintragung nicht darauf vertrauen, entsprechende Beschränkungen der Geschäftsfähigkeit existierten nicht.

\section{bb) Feblende oder beschränkte Verfügungsbefugnis}

Bestehen auf Seiten des Veräußerers Verfügungshindernisse in Gestalt von Verfügungsverboten oder -beschränkungen, ist zwischen relativen und absoluten Verfügungsverboten zu differenzieren. Für relative Verfügungsverbote verweist $\$ 26$ Satz $2 \mathrm{eWpG}$ auf die Eintragungspflichten aus $\$ 13$ Abs. 2 Satz 1 Nr.1, $\mathbb{1} 17$ Abs. 2 Satz 1 Nr. 1 eWpG. Aus den zugehörigen Spezialregelungen (z. B. $\$ 135$ Abs. 2, $\$ 161$ Abs. 3, \$2113 Abs. 3 BGB) folgt die Möglichkeit eines gutgläubigen Erwerbs. Demgegenüber unterfallen absolute Verfügungsverbote den „sonstigen Verfügungsbeschränkungen“ i. S. d. \13 Abs. 2 Satz 2, \17 Abs. 2 Satz $3 \mathrm{eWpG}$, so dass nach $\$ 26$ Satz $3 \mathrm{eWpG}$ insofern ein gutgläubiger Erwerb ausscheidet.

\section{cc) Feblende Bevollmächtigung}

Anders als noch im Referentenentwurf ( $\$ 26$ Nr. 3 eWpG-RefE) vorgesehen, ${ }^{69}$ wird der gute Glaube an die rechtsgeschäftliche oder gesetzliche Vertretungsmacht nicht spezialgesetzlich geschützt. Es bleibt bei den allgemeinen Regeln der BGB-Rechtsgeschäftslehre, insbesondere $\$ \int 170,172$ BGB. Grundsätzlich kann von einem Vertreter ohne Vertretungsmacht daher nicht erworben werden.

\section{d) Rechtsverhältnis Berechtigter-Erwerber}

Soweit der Tatbestand des gutgläubigen Erwerbs erfüllt ist und der Erwerber zum Eigentümer des elektronischen Wertpapiers wird, steht dem vorherigen Eigentümer kein Herausgabeanspruch gegen den neuen Eigentümer zu. Ein solcher Vindikationsanspruch aus $\$ 985$ BGB i. V.m. $\$ 2$ Abs. 3 eWpG setzt das (fortbestehende) Eigentum des Anspruchsinhabers voraus. Scheitert hingegen der gutgläubige Erwerb und wird der Erwerber dennoch als Inhaber in das Register eingetragen, steht dem Berechtigten ein Vindikationsanspruch zu. Die Herausgabe erfolgt durch Mitwirkung an der Registereintragung des Berechtigten, d.h. durch Erteilung einer entsprechenden Weisung ( $\$ 14$ Abs. 1 Satz 1 Nr. 1, \18 Abs. 1 Satz 1 Nr. 1 eWpG). Eine Rückübertragung ist hingegen weder erforderlich noch möglich, da eine wirksame Übertragung zuvor nicht stattgefunden hat. ${ }^{70}$ Ein Registerberichtigungsanspruch nach dem Vorbild von $\ 894$

\footnotetext{
69 Dazu Sickinger/Thelen, AG 2020, 862 Rn. 34 ff.; Linardatos, ZBB 2020, 329, 343.

70 Ungenau daher Casper, in: Möslein/Omlor (Hrsg.), FinTech-Handbuch (2. Aufl. 2021), §28 Rn. 53.
} 
BGB existiert hingegen nicht. ${ }^{71}$ Stattdessen muss auf $\mathbb{S} \mathbb{S} 14,18$ eWpG zurückgegriffen werden.

\section{Sammeleintragung}

\section{Grundlagen}

Aus den eWpG-Sonderregeln werden Verfügungen über elektronische Wertpapiere in Sammeleintragung weitgehend ausgeklammert. Abschnitt 4 des eWpG bezieht sich bereits nach seiner amtlichen Überschrift nur auf elektronische Wertpapiere in Einzeleintragung. Daher finden die dortigen Vorschriften keine Anwendung auf Verfügungen über einen Wertpapiersammelbestand. ${ }^{72}$ Entsprechend verweist $\$ 9$ Abs. 1 Satz $1 \mathrm{eWpG}$ auf die depotrechtlichen Regelungen. Elektronische Wertpapiere gelten nunmehr ebenfalls als Wertpapiere i.S.d. DepotG ( $\$ 1$ Abs. 1 Satz 3 DepotG). Weitgehend finden die bestehenden Vorgaben zur Sammelverwahrung entsprechend für elektronische Wertpapiere Anwendung ( $\mathbb{9} 9 \mathrm{~b}$ Abs. 1 Satz 1 DepotG). ${ }^{73}$ Die Verfügung über elektronische Wertpapiere richtet sich daher je nach Eintragungsform unterschiedlichen Regelungsregimen. ${ }^{74}$

\section{2. Übergabe von elektronischen Wertpapieren}

Nach traditioneller und überwiegender Ansicht ${ }^{75}$ erfolgt die Übertragung von sammelverwahrten Wertpapieren nach $\$ \mathbb{S} 929 \mathrm{ff}$. BGB, worauf $\$ 24$ Abs. 2 Satz 1 DepotG im letzten Halbsatz verweist. Umstritten ist daher, ob und nach welcher Vorschrift die Übergabe erfolgt, insbesondere bei einem Ausschluss des Anspruchs auf Einzelverbriefung ( $\$ 9$ a Abs. 3 Satz 2 DepotG) ${ }^{76}$ Die besitzrechtliche Belastbarkeit der herrschenden Meinung, die eine Übergabe bzw. ein Übergabesurrogat annimmt, ${ }^{77}$ wird jedoch durch die Zwischenschaltung einer Sachfiktion (weiter) erschüttert. Die fragile Besitzkonstruktion entfernt sich bei einer körperlosen Sache, bei welcher an die Stelle der tatsächlichen

71 Casper, in: Möslein/Omlor (Hrsg.), FinTech-Handbuch (2. Aufl. 2021), $\$ 28$ Rn. 53.

72 BT-Drs. 19/26925, S. 65; Bialluch-von Allwörden, RDi 2021, 13 Rn. 11.

73 Linardatos, ZBB 2020, 329, 340; begrüßend Döding/Wentz, WM 2020, 2312, 2315f.; Preuße/Wöckener/Gillenkirch, BKR 2020, 551, 554.

${ }^{74}$ Kritisch Bialluch-von Allwörden, RDi 2021, 13 Rn. 11.

75 Stellvertretend BGH, Beschl. v. 16.07.2004, Az. IXa ZB 24/04 = BGHZ 160, 121, 124; BGH, Urt. v. 30.11.2004, Az. XI ZR 200/03 = BGHZ 161, 189, 191 ff.; BGH BKR 2013, 334 Rn.14; Segna, Bucheffekten (2018), S. 207 ff. m.w. N.; a. A. Einsele, Wertpapierrecht als Schuldrecht (1995), S.64ff.; Habersack/Mayer, Globalverbriefte Aktien als Gegenstand sachenrechtlicher Verfügungen?, WM 2000, 1678, $1681 \mathrm{ff}$.

${ }^{76}$ Zum Meinungsstand vgl. Lehmann, Finanzinstrumente (2010), S. $388 \mathrm{ff}$.; MüKo/Einsele, HGB (4. Aufl. 2019), Bankvertragsrecht Q. Depotgeschäft Rn. $110 \mathrm{ff}$.

77 Beispielhaft BGH, Beschl. v. 16.07.2004, Az. IXa ZB 24/04 = BGHZ 160, 121, 124. 
Sachherrschaft weitgehend der Registerbesitz gesetzt wurde (vgl. $\$ \$ \$ 24,25$ Abs. 1 eWpG), ${ }^{78}$ sichtbar von den sachenrechtlichen Grundstrukturen. Daher erscheint es vorzugswürdig, zumindest für elektronische Wertpapiere in Sammeleintragung zur Übereignung auf eine Übergabe oder ein Übergabesurrogat zu verzichten und eine dingliche Einigung ausreichen zu lassen. ${ }^{79}$ Auch ein gutgläubiger Erwerb von elektronischen Wertpapieren in Sammelverwahrung ist möglich und richtet sich nach $\$ 2$ Abs. 3, $\$ 9$ Abs. 1 eWpG i. V.m. $\$ 932$ BGB. ${ }^{80}$ Diffus erscheint der taugliche Rechtsscheinträger. ${ }^{81}$ De lege ferenda sollte eine Anpassung an die Übertragung von elektronischen Wertpapieren in Einzeleintragung erfolgen, so dass die Übergabe durch eine Eintragung in das elektronische Wertpapierregister surrogiert würde.

Anders als bei nicht-elektronischen Wertpapieren droht bei einem Verzicht auf die Übergabe für eine Pfandrechtsbestellung kein besitzloses Pfandrecht, ${ }^{82}$ da ein Besitz i.S.d. $\$ 1205$ BGB an einem elektronischen Wertpapier nicht besteht. Daher kann in Parallele zur Eigentumsübertragung auch eine Verpfändung von elektronischen Wertpapieren in Sammeleintragung durch schlichte Einigung erfolgen. Einen gutgläubigen Pfandrechtserwerb vom Nichtberech-

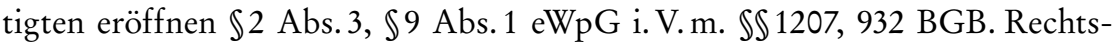
politisch begegnet das Konzept hingegen ebenfalls Zweifeln im Hinblick auf die angestrebte Verfügungstransparenz $(\$ 24 \mathrm{Nr} .3 \mathrm{eWpG})$, so dass eine konstitutive Registereintragung wie in $\$ 25$ Abs. $1 \mathrm{eWpG}$ verlangt werden sollte.

\section{Zusammenfassung}

1. Die pauschale Sachfiktion des $\$ 2$ Abs. 3 eWpG führt im BGB-Sachenrecht zu Anwendungsproblemen, die sich insbesondere auf die Fiktion des Besitzes und die Abgrenzung zwischen Mobiliar- und Immobiliarsachenrecht beziehen.

2. Welche sachenrechtlichen Vorschriften auf elektronische Wertpapiere Anwendung finden, sollte im Einzelfall nach der jeweiligen Teleologie der konkreten BGB- und eWpG-Normen entschieden werden. Der Weg in das Immobiliarsachenrecht ist nicht a priori verschlossen, da mit dem Grundbuch und dem elektronischen Wertpapierregister jeweils ein künstlicher Rechtsscheinträger zur Verwendung kommt.

78 Lebmann, BKR 2020, 431, 436.

79 Casper, in: Möslein/Omlor (Hrsg.), FinTech-Handbuch (2. Aufl. 2021), \$28 Rn.48; ebenso generell Einsele, Wertpapierrecht als Schuldrecht (1995), S.90f.; a. A. wohl Döding/ Wentz, WM 2020, 2312, 2316; Segna, Elektronische Wertpapiere im zentralen Register, WM 2020, 2301, 2308: \$S $929 \mathrm{ff} . \mathrm{BGB}$.

80 Casper, in: Möslein/Omlor (Hrsg.), FinTech-Handbuch (2. Aufl. 2021), \$28 Rn. $49 \mathrm{f}$.

81 Ähnlich Segna, WM 2020, 2301, 2309.

82 Vgl. zu nicht-elektronischen Wertpapieren Habersack/Mayer, WM 2000, 1678, 1684. 
3. Verfügungen über elektronische Wertpapiere in Einzeleintragung sind konstitutiv an eine Registereintragung geknüpft („keine Verfügung außerhalb des Registers").

4. Eine Abtretung der verbrieften Forderung ( $\mathbb{S} 398 \mathrm{ff}$. BGB) bleibt bei einer Einzeleintragung möglich, so dass nach $\$ 952$ Abs. 2 BGB das Eigentum am elektronischen Wertpapier nachfolgt. Jedoch bedarf es für die Wirksamkeit der Abtretung einer konstitutiven Registereintragung.

5. Die Weisung i.S.d. $\$ \mathbb{S 1 4}$ Abs. 1, 18 Abs. 1,25 Abs. $1 \mathrm{eWpG}$ stellt eine Willenserklärung dar, die rechtsgedanklich in Teilen auf das subjektive Element der Übergabe i.S.d. $\$ 929$ Satz 1 BGB und auf die grundbuchrechtliche Bewilligung ( $\$ 19 \mathrm{GBO})$ zurückgeht.

6. Der gutgläubige Erwerb nach $\$ 26 \mathrm{eWpG}$ basiert auf dem öffentlichen Glauben des elektronischen Wertpapierregisters und ähnelt daher stärker $\$ 892$ BGB als $\$ 932$ BGB. Geschützt wird das abstrakte Vertrauen auf den gesetzlich vorgeschriebenen Registerinhalt. Ein gutgläubig-lastenfreier Erwerb ist möglich.

7. Anstelle eines Registerberichtigungsanspruchs nach dem Vorbild von \$894 BGB steht dem Eigentümer eines elektronischen Wertpapiers gegen den Inhaber ein Vindikationsanspruch ( $\$ 985$ BGB i. V. m. $\$ 2 ~ A b s .3 \mathrm{eWpG}$ ) zu, der auf Mitwirkung an der Registerberichtung gerichtet ist.

8. Die Verfügung über elektronische Wertpapiere in Sammeleintragung richtet sich nach den depotrechtlichen Vorgaben, nicht nach Abschnitt 4 des eWpG. Für die Übertragung nach $\$ 929$ Satz 1 BGB bedarf es ausnahmsweise keiner Übergabe, da die Sachfiktion insofern keine hinreichend belastbare Besitzfiktion umfasst. 


\title{
Aufsicht und Sanktionen
}

\author{
Julia von Buttlar ${ }^{1}$
}

I. Zielsetzungen und Regelungsansatz . . . . . . . . . . . . . . . . 159

II. Kryptowertpapierregisterführung als Finanzdienstleistung . . . . . . . 161

1. Aufsichtsrelevante Tätigkeiten und Ausnahmetatbestände ......... 162

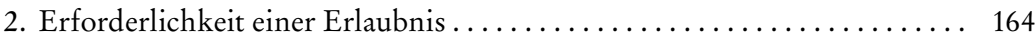

3. Einschreiten bei Verstößen oder unerlaubten Geschäften . .......... 166

4. Strafbares Handeln ohne Erlaubnis . . . . . . . . . . . . . . . . . . . . 167

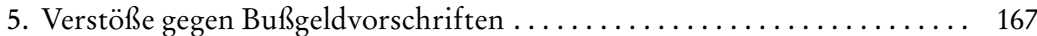

III. Aufsicht über die ordnungsgemäße Führung von Kryptowertpapier-

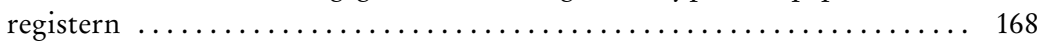

1. Funktionale und technische Anforderungen ................ 169

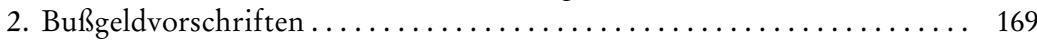

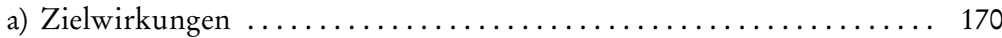

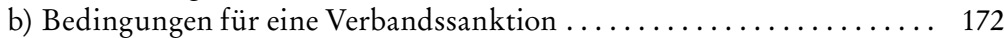

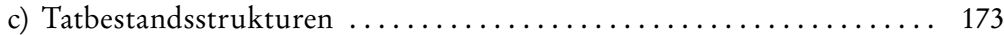

IV. Elektronische Wertpapiere und Marktaufsicht . . . . . . . . . . . . . 174

V. Ausblick ......................................... 175

Das eWp ist ein großer Schritt in Richtung vollständig dematerialisierter Wertpapiere: Deutschland macht mit seiner Blockchain-Strategie in Europa ernst. Es ist wahrscheinlich, dass die entstehenden Blockchain-Ökosysteme im nächsten Jahrzehnt einer der stärksten Game-Changer für Finanz- und Rechtsdienstleistungen sein werden. Insbesondere einige europäische Länder haben erhebliche Fortschritte in Bezug auf die Nutzung und Unterstützung von DLT und Blockchain gemacht. ${ }^{2}$ Die Blockchain-Technologie wird in verschiedenen Bereichen eingesetzt, darunter auch im Finanzsektor. Im Zusammenhang mit der Blockchain-Technologie werden derzeit in Europa und speziell in Deutschland verschiedene Gesetzesvorhaben vorangetrieben.

${ }^{1}$ Die folgenden Ausführungen geben ausschließlich die persönliche Auffassung der Autorin wieder.

${ }^{2}$ Zur Rechtslage in der Schweiz, vgl. Weber, Neue Blockchain-Gesetzgebung in der Schweiz, RDi 2021, $186 \mathrm{ff}$. 
Im August 2020 hat das BMF den Referentenentwurf ${ }^{3}$ eines Gesetzes zur Einführung von elektronischen Wertpapieren (Art.1: Gesetz über elektronische Wertpapiere, „eWpG“) veröffentlicht. ${ }^{4}$ Am 16. Dezember 2020 hat die Bundesregierung den Gesetzesentwurf ${ }^{5}$ auf der Webseite des BMJV bekannt gemacht und am 24.02.2021 vorgelegt. ${ }^{6}$ Am 10.06.2021 ist das Gesetz in Kraft getreten. ${ }^{7}$ Zentrale Botschaft des eWpG ist nicht eine umfassende Reform des deutschen Wertpapierrechtes, sondern eine Einbettung der elektronischen Begebung von bestimmten Wertpapieren in die bestehende Regelungssystematik. Die im eWpG vorgesehenen materiell-rechtlichen Änderungen sind eng miteinander verbunden und wirken sich auf das Zivilrecht, auf das Verwaltungsrecht, auf das Europarecht sowie auf das Straf- und Ordnungswidrigkeitenrecht aus. Das eWpG kann in diesem Sinne auch unter metaphorischer Zuhilfenahme der olympischen Ringe als ein Gemeinschaftsprojekt verschiedenster Rechtsgebiete verstanden werden, die möglichst friktionslos ineinander greifen müssen, um einem solch ambitionierten Projekt zu Erfolg verhelfen zu können.

Die Öffnung soll sich dabei zunächst auf elektronische Schuldverschreibungen und Anteilscheine an Sondervermögen nach dem KAGB beschränken. Die Regulierung elektronischer Wertpapiere soll technologieneutral erfolgen, d.h. die Begebung elektronischer Wertpapiere soll auch auf Grundlage der Distributed Ledger-Technologie (DLT), deren bekanntester Vertreter die Blockchain ist, möglich sein. Der Entwurf unterscheidet zwischen der Führung eines zentralen elektronischen Wertpapierregisters durch einen Zentralverwahrer sowie der

3 Abrufbar unter https://www.bmjv.de/SharedDocs/Gesetzgebungsverfahren/Doku mente/RefE_Einfuehrung_elektr_Wertpapiere.html;jsessionid=87F7BB5F245A2D2087866 A71995445B8.2_cid297?nn=6712350, im Folgenden: RefE (alle Hyperlinks in diesem Beitrag wurden zuletzt am 14.05.2021 abgerufen).

${ }^{4}$ Vgl. hierzu Lehmann, Zeitenwende im Wertpapierrecht, BKR 2020, 431 ff.; Saive, Einführung elektronischer Wertpapiere, ZRP 2020, $219 \mathrm{ff} . ;$ Preuße/Wöckener/Gillenkirch, Der Gesetzesentwurf zur Einführung elektronischer Wertpapiere, BKR 2020, 551 ff.; Segna, Elektronische Wertpapiere im zentralen Register - Anmerkungen zum BMF-/BMJV-Referentenentwurf vom 10.8.2020 aus wertpapier- und depotrechtlicher Sicht, WM 2020, $2301 \mathrm{ff}$.; $D \ddot{o}-$ ding/Wentz, Der Referentenentwurf zur Einführung von elektronischen Wertpapieren und Kryptowertpapieren, WM 2020, $2312 \mathrm{ff}$.; Linardatos, Elektronische Schuldverschreibungen auf den Inhaber - des Wertpapiers neue Kleider, ZBB 2020, 329 ff.; Sickinger/Thelen, Anleihen und Genussscheine auf der Blockchain, AG 2020, $862 \mathrm{ff}$.

${ }_{5}$ Abrufbar unter: https://www.bmjv.de/SharedDocs/Gesetzgebungsverfahren/DE/Ein fuehrung_elektr_Wertpapiere.html.

${ }^{6}$ BT-Drucks. 19/26025, abrufbar unter https://dip21.bundestag.de/dip21/btd/19/269/ 1926925.pdf., im Folgenden: RegE; vgl. hierzu Bialluch-von Allwörden, Zivil- und prospektrechtliche Aspekte des eWpG-E, RDi 2021, 13 ff.; Meier, Übertragung von elektronischen Wertpapieren nach dem eWpG-E, RDi 2021, 1 ff.; Labusen, Das Sachenrecht der elektronischen Wertpapiere, RDi 2021, 161 ff.; Mittwoch, Der Entwurf eines Gesetzes zur Einführung elektronischer Wertpapiere - ein Quantensprung für das Zivil- und Finanzmarktrecht?, WM 2021, $375 \mathrm{ff} . ;$ Vo $\beta$, Der Regierungsentwurf des eWpG und das Depotrecht - Ein Warnruf, ZDiW, 2021, $16 \mathrm{ff}$.

7 BGBl. 2021 S. 1423. 
Führung von unter anderem durch DLT ermöglichten Registern zur Begebung elektronischer Schuldverschreibungen. Sog. Kryptowertpapiere können ohne Abbildung im Effektengiro auf einem in der Regel DLT-basierten Kryptowertpapierregister begeben und verwaltet werden. Bei dem Schritt, Anlegern den Erwerb und Handel von Kryptowertpapieren zu ermöglichen, zeigt sich ganz besonders das innovative Potential des Gesetzgebungsentwurfs.

Dieser Beitrag widmet sich in Teil I den aufsichtsrechtlichen Eingriffsbefugnissen, die aus der der Aufnahme der Kryptowertpapierregisterführung als eine neue Finanzdienstleistung in das KWG folgen. Teil II stellt dar, welche Aktivitäten erlaubnispflichtig sind und welche Rechtsfolgen bei Verletzung drohen. Teil III analysiert die Eingriffsbefugnisse im Fall einer Nichtbeachtung der besonderen Anforderungen und Pflichten für die Finanzdienstleistung Kryptowertpapierregisterführung. Inwieweit die Regeln des kapitalmarktrechtlichen Sekundärrechts, als das Recht des Wertpapierhandels, auf elektronische Wertpapiere Anwendung finden, ist Gegenstand von Teil IV. Der Beitrag endet in Teil V mit einem Ausblick.

\section{Zielsetzungen und Regelungsansatz}

Durch den Gesetzesentwurf sollen die Voraussetzungen für Innovationen im Finanzsektor verbessert und der Finanzplatz Deutschland gestärkt werden. ${ }^{8}$ Die Emission von Krypto-Token und gerade von Kryptowertpapieren hat grundsätzlich das Potenzial, sich zu einer neuen Finanzierungsform von insbesondere neuen bzw. jungen sowie kleinen und mittleren Unternehmen (Startups, KMU) zu entwickeln, sofern ein ausreichender Anlegerschutz und Vertrauen in diese Art der Refinanzierung hergestellt werden können. ${ }^{9}$

Aus Anlegersicht werden die Funktionsweisen der jeweiligen Kryptowertpapiere anhand des zugrundeliegenden Programmiercodes (Smart Contract) schwer zu überprüfen sein. Der Code kann sich zudem als angreifbar und damit manipulierbar erweisen. Die systembedingte Anfälligkeit für Betrug, Geldwäsche und Terrorismusfinanzierung erhöht das Risiko, dass Anleger das eingesetzte Kapital verlieren, auch aufgrund notwendiger Maßnahmen der Behörden gegen Betreiber oder sonstige Personen und Unternehmen, die in solche illegalen Geschäfte einbezogen sind. ${ }^{10}$

8 BT-Drucks. 19/26925, S. 40.

9 BMF-Monatsbericht, Juni 2019, S.2 abrufbar unter https://www.bundesfinanzmi nisterium.de/Monatsberichte/2019/06/Inhalte/Kapitel-3-Analysen/3-1-krypto-token.html.

10 Vgl die Verbaucherwarnung der BaFin zu ICOs vom 09.11.2017, abrufbar unter https:// www.bafin.de/SharedDocs/Veroeffentlichungen/DE/Meldung/2017/meldung_171109_ ICOs.html. 
$\mathrm{Zu}$ Recht hat daher schon das Eckpunktepapier darauf verwiesen, dass eine der zentralen Fragen sein wird, wie der Anlegerschutz bei elektronischen Wertpapieren sichergestellt werden kann, insbesondere bei Führung des Wertpapierregisters auf einer Blockchain durch den Emittenten oder einen von ihm mit der Führung beauftragten Dritten. ${ }^{11}$

Dem Anlegerschutz und der Marktintegrität sowie der Transparenz und dem Funktionsschutz der Kapitalmärkte bei über Blockchain begebenen Wertpapieren soll insbesondere auch dadurch Rechnung getragen werden, dass das Führen eines Kryptowertpapierregisters unter die Aufsicht durch die Bundesanstalt für Finanzdienstleistungsaufsicht (BaFin) gestellt wird. ${ }^{12}$ Die Alternative wäre eine detaillierte technische Regulierung insbesondere der Kryptowertpapierregister gewesen. Aufgrund der großen Innovationskraft und -geschwindigkeit digitaler Technologien bestehen gegenüber diesem Ansatz jedoch große Bedenken. ${ }^{13}$ Die staatliche Aufsicht soll dazu führen, dass flexibler und kurzfristiger auf neue Entwicklungen reagiert werden kann. Die Beaufsichtigung soll das Manipulationsrisiko durch den das Kryptowertpapierregister führenden Emittenten verringern und hierdurch Anlegerschutz gewährleisten.

Kryptowertpapierregisterführer werden künftig weitgehend nach den gleichen Regeln beaufsichtigt wie Kredit- und Finanzdienstleistungsinstitute und haben bestimmte Ordnungsvorschriften und Verhaltenspflichten zu beachten, die zum Zwecke des Anlegerschutzes nach dem Kreditwesengesetz (KWG) und dem Wertpapierhandelsgesetz (WpHG) gelten. In diesem Zusammenhang ist anzumerken, dass die BaFin nach $\$ 4$ Abs. 1 a FinDAG ausschließlich auf den kollektiven und nicht den individuellen Verbraucherschutz verpflichtet ist. ${ }^{14} \mathbb{} \int 4$ Abs. 4 FinDAG bestimmt, dass die BaFin ihre Aufgaben im öffentlichen Interesse wahrzunehmen hat. Allgemein kann man sagen, dass es zu den Aufgaben der BaFin gehört, Gefahren vom Kapitalmarkt und dem Finanzplatz Deutschland im öffentlichen Interesse abzuwenden. ${ }^{15}$ Mit dem Funktionsschutz des Kapitalmarktes geht letztlich als Rechtsreflex der Schutz der Anleger einher. Letztendlich hängt die Funktionsfähigkeit des Marktes - wie bereits erwähnt - doch maßgeblich davon ab, ob die Anleger als Investoren Kapital zur Verfügung stellen, weil sie Vertrauen in die Funktionsfähigkeit und die Schutzmechanismen

${ }^{11}$ Eckpunkte für die regulatorische Behandlung von elektronischen Wertpapieren und Krypto-Token vom 19.03.2019, S.5 abrufbar unter https://www.bundesfinanzministerium. de/Content/DE/Gesetzestexte/Gesetze_Gesetzesvorhaben/Abteilungen/Abteilung_VII/ 19_Legislaturperiode/2019-03-07-Eckpunktepapier-Wertpapiere-Krypto-Token/2019-0307-Eckpunktepapier-regulatorische-Behandlung-elektronische-Wertpapiere-Krypto-To ken.pdf?__blob=publicationFile\&v=3.

12 BT-Drucks. 19/26925, S. 1.

13 So Preuße/Wöckener/Gillenkirch, BKR 2020, S. 551, 512.

14 Siehe auch OLG Frankfurt BKR 2020, 597 ff. Rn. 34 [= Rn. 38 nach juris], anhängig beim BGH unter AZ: III ZR 41/20; Gesetzentwurf der Bundesregierung BT-Drucks. 18/3994, 37.

15 Vgl. $₫ 4$ Abs. 4 FinDAG. 
des Wertpapiermarktes haben, sie - mit anderen Worten - vor einem Versagen des Markts hinreichend geschützt sind. Andernfalls können die Konsequenzen für den Kapitalmarkt verheerend sein, wenn das Vertrauen der Anleger darauf, dass sie am Markt nicht irregeführt werden, verloren geht.

\6 KWG begründet die Zuständigkeit der BaFin für die laufende Überwachung des Geschäftsbetriebs von Finanzdienstleistungsinstituten und stattet die BaFin mit den hierfür notwendigen Einsichtnahme- und Anordnungsbefugnissen aus, ebenso wie nach $\$ 6 \mathrm{WpHG}$ für die Aufsicht über die Ordnungsmäßigkeit des Wertpapierhandels. Zur Ordnungsmäßigkeit der Geschäfte und des Handels gehört die Einhaltung des einschlägigen geltenden Rechts.

Der im Kreditwesengesetz verankerten Solvenzaufsicht stehen als Aufsichtsinstrumente schwerpunktweise die Zulassung von Kredit- bzw. Finanzinstituten zum Geschäftsbetrieb, Ordnungsvorschriften für den Geschäftsbetrieb, die laufende Überwachung der wirtschaftlichen Situation der Institute, die Überprüfung der Zuverlässigkeit und der fachlichen Eignung der Geschäftsleiter, die Anteilseignerkontrolle sowie die repressive Verfolgung unerlaubt betriebener Bank- oder Finanzdienstleistungsgeschäfte zur Verfügung. Die im WpHG geregelte Marktaufsicht bezweckt hingegen insbesondere die Bekämpfung von Insidergeschäften und Marktmanipulation, die Kontrolle der Ad-hoc-Publizität, die Überwachung von Mitteilungs- und Prospektveröffentlichungspflichten sowie die Überwachung der Einhaltung der Organisationsvorschriften. Das eWpG enthält Vorschriften, die dem Zivilrecht zuzuordnen sind, aber auch Vorschriften zur Solvenz- und Marktaufsicht.

Gefahren oder marktschädigendem Verhalten kann die BaFin im Rahmen der Solvenz- und Marktaufsicht auf zwei Wegen entgegenwirken. Zum einen kann im Rahmen eines Verwaltungsverfahrens angeordnet werden, das beanstandete Verhalten zu beenden. Dazu können auch Zwangsmittel eingesetzt werden. Zum anderen kann im Wege eines Ordnungswidrigkeitenverfahrens eine Geldbuße verhängt werden.

\section{Kryptowertpapierregisterführung als Finanzdienstleistung}

Mit dem neuen $\ 1$ Abs. 1a S. 2 Nr. 8 KWG-E wird die Kryptowertpapierregisterführung als neue Finanzdienstleistung geschaffen. Das KWG unterscheidet je nach Art der Finanzdienstleistung zwischen mehreren Typen von Finanzdienstleistungsinstituten, für die dann unterschiedlich strenge Ordnungsvorschriften und Verhaltenspflichten zur Anwendung kommen. Gesetzestechnisch gesehen geschieht dies in Form einer Vielzahl sehr unterschiedlicher Ausnahme- und Befreiungsvorschriften, bei denen es in der Praxis schwer fällt den Überblick zu behalten. Diese Gesetzestechnik gilt auch für die Kryptowertpapierregisterführung. 


\section{Aufsichtsrelevante Tätigkeiten und Ausnabmetatbestände}

Die Finanzdienstleistung nach $₫ 1$ Abs. 1a S. 2 Nr. 8 KWG-E erfasst die Führung eines Kryptowertpapierregisters nach $\$ 16 \mathrm{eWpG}$. Emittenten können Kryptowertpapierregister entweder durch einen Dienstleister oder selbst führen und müssen die Wertpapiere nicht wie bislang aufwendig bei einem Zentralverwahrer, wie Clearstream in Deutschland, verwahren. Sie werden den Wertpapieren gleichgestellt, aber dennoch technologieneutral ausgestaltet. Eine Festlegung auf die Distributed Ledger-Technologie oder eine bestimmte Ausprägung dieser Technik sei nicht bezweckt. ${ }^{16}$ Nach dem derzeitigen Stand der Technik würden laut der Gesetzesbegründung hier in erster Linie Aufzeichnungssysteme auf Basis der DLT in Betracht kommen. ${ }^{17}$ Emittenten können daher Kryptowertpapiere künftig zum Beispiel auf dezentralen Blockchains als Unterfall der Distributed Ledger-Technologie emittieren. Kryptowertpapiere werden durch Software automatisiert verwaltet. Das erlaubt, Regeln für die Verwaltung, die Emission und den Handel von Kryptowertpapieren in Form von sogenannten Smart Contracts zu programmieren.

Der Anwendungsbereich des Kryptoverwahrgeschäfts wird in $\$ 1 \mathrm{Abs.1a}$ S. 2 Nr. 6 KWG erweitert, um die Sicherung von privaten kryptographischen Schlüsseln, die dazu dienen, Kryptowertpapiere für andere nach $\mathbb{} \$ 4 \mathrm{Abs} .3$ eWpG zu halten, zu speichern oder zu verfügen. Die Verwahrung und Verwaltung von Kryptowertpapieren fällt dagegen nunmehr unter das Depotgeschäft, sodass es hier keiner Ergänzung bedarf. ${ }^{18}$ Wenn ein Unternehmen am Tag des Inkrafttretens des eWpG über eine Erlaubnis für den Betrieb des Kryptoverwahrgeschäfts verfügt, kann es dieses Geschäft auch hinsichtlich der Sicherung von privaten kryptografischen Schlüsseln erbringen, die dazu dienen, Kryptowertpapiere nach $\$ 4 \mathrm{Abs.} 2 \mathrm{eWpG}$ zu halten, zu speichern oder darüber zu verfügen. ${ }^{19}$

Für Finanzdienstleistungsinstitute, die außer dem Kryptoverwahrgeschäft oder der Kryptowertpapierregisterführung keine weiteren Finanzdienstleistungen im Sinne des $\$ 1$ Abs. 1a S. 2 erbringen, gilt die Ausnahmevorschrift des $\$ 2 \mathrm{Abs}$. 7b KWG. Danach sind bei Finanzdienstleistungsinstituten, die allein die Kryptowertpapierregisterführung betreiben, die $\$ \mathbb{1 0}$, 10c bis 18 und $24 \mathrm{Abs} .1$ Nummer 14 bis $14 b$, die $\$ \$ 24 a$ und 25 a Abs. 5, die $\$ \$ 26 a$ und 45 KWG sowie die Artikel 39, 41, 50 bis 403 und 411 bis 455 der Verordnung (EU) Nr. 575/2013 nicht anzuwenden. Anwendbar bleiben jedoch die Regelungen für den Inhaber bedeutender Beteiligungen gem. $\$ 2 \mathrm{c}$ KWG, die Meldepflichten gemäß $\$ 24$

\footnotetext{
16 BT-Drucks. 19/26925, S. 74.

17 BT-Drucks. 19/26925, S. 74.

${ }_{18}$ Rennig, in: Voß/Rennig (Hrsg.), Recht der FinTechs, Kryptowerte im Aufsichts- und Geldwäscherecht (in Vorbereitung für 2022).

19 Vgl. $\$ 65$ KWG.
} 
KWG, die besonderen organisatorischen Pflichten gem. \$25a KWG (konkretisiert durch MaRisk und BAIT), die Vorlage eines Jahresabschlusses, die Durchführung der jährlichen Prüfung gem. $\$ \$ \$ 26,28 \mathrm{KWG}$ sowie die Regelungen zum Anfangskapital. Im Vergleich zum Referentenentwurf vom 11.08.2020 ist bei der vorliegenden Fassung vom 16.12.2021 zu begrüßen, dass das erforderliche Anfangskapital angepasst worden ist. Der Blockchain-Markt wird maßgeblich von Start-ups entwickelt, geprägt und vorangetrieben. Der Blockchain Bundesverband hatte kritisiert, dass der Referentenentwurf diesem Sachverhalt durch die vorgesehenen sehr hohen finanziellen und regulatorischen Anforderungen nicht gerecht werde und diese Start-ups strukturell vom Markt der elektronischen Wertpapiere aussperre. ${ }^{20}$ In seiner Stellungnahme vom 14.09.2020 hatte er eine Absenkung der Anfangskapitalanforderungen in Höhe von 750.000 EUR gefordert, um die Innovationskraft von Start-ups für die weitere Entwicklung des Marktes nutzbar zu machen. ${ }^{21}$

Gem. \33 Abs. 1 S. 1 Nr.1 b) KWG ist nunmehr ein Anfangskapital von 125.000 EUR wie für das vergleichbare Kryptoverwahrgeschäft vorzuhalten. Zur Begründung der Absenkung führt der Regierungsentwurf aus, dass die Tätigkeit der registerführenden Stelle im Kern ebenso wie das Kryptoverwahrgeschäft eine innovative, digitale Dienstleistung sei, für die damit einhergehend aufgrund des vergleichbaren Risikoprofils und den inhärenten Sicherheitsaspekten der Distributed Ledger Technologie die gleiche Höhe des Anfangskapitals gelten soll.22

Zudem finden auch geldwäscherechtliche Vorgaben ${ }^{23}$ und Vorschriften zu internen Sicherungsmaßnahmen ( $\$ 25 \mathrm{~h} \mathrm{KWG)} \mathrm{sowie} \mathrm{zu} \mathrm{verstärkten} \mathrm{Sorg-}$ faltspflichten $(\$ 25 \mathrm{k}$ KWG) Anwendung. Die Pflichten erstrecken sich über die Etablierung eines hinreichenden Risikomanagements bis zu spezifischen Sorgfaltspflichten in Bezug auf Kunden und ergänzen die fachgesetzlichen Compliance-Anforderungen im Bereich der Geldwäscheprävention.

Der Tatbestand der Kryptowertpapierregisterführung ( $\$ 1$ Abs. 1a S. 2 Nr. 8 KWG) - als rein nationale Regelung - ist hingegen keine Wertpapierdienstleistung - weder im Sinne des WpIG-E noch nach WpHG/MiFID II. Wertpapier-

20 Vgl. Stellungnahme des Blockchain Bundesverbandes vom 14.09.2020, S. 7, abrufbar unter https://www.bundesfinanzministerium.de/Content/DE/Gesetzestexte/Gesetze_Geset zesvorhaben/Abteilungen/Abteilung_VII/19_Legislaturperiode/2020-08-11-einfuehrungelektronische-wertpapiere/Stellungnahme-blockchain.pdf?__blob=publicationFile\&v=1.

$21 \mathrm{Vgl}$. Stellungnahme des Blockchain Bundesverbandes vom 14.09.2020, S. 7, abrufbar unter https://www.bundesfinanzministerium.de/Content/DE/Gesetzestexte/Gesetze_Geset zesvorhaben/Abteilungen/Abteilung_VII/19_Legislaturperiode/2020-08-11-einfuehrungelektronische-wertpapiere/Stellungnahme-blockchain.pdf?_blob=publicationFile\&v=1.

22 BT-Drucks. 19/26925, S. 74.

23 Als Finanzdienstleistungsinstitut im Sinne des KWG ist der Betreiber der Kryptowertpapierregisterführung zugleich geldwäscherechtlich Verpflichteter im Sinne des \$2 Abs. 1 Nr. 2 GwG. 
dienstleistungsunternehmen sind gemäß $\$ 2$ Abs. 10 WpHG Kreditinstitute, Finanzdienstleistungsinstitute und nach $\$ 53$ Abs. 1 S. 1 KWG tätige Unternehmen, die Wertpapierdienstleistungen allein oder zusammen mit Wertpapiernebendienstleistungen gewerbsmäßig oder in einem Umfang erbringen, der einen in kaufmännischer Weise eingerichteten Geschäftsbetrieb erfordert. Kryptowertpapierregisterführer sind damit keine Wertpapierdienstleistungsunternehmen oder Wertpapierdinstleistungsinstitute.

\section{Erforderlichkeit einer Erlaubnis}

Der Führung eines Kryptowertpapierregisters steht gem. \32 Abs. 1 S. 1 KWG unter einem präventiven Verbot mit Erlaubnisvorbehalt. Diese Finanzdienstleistung führt gemäß $\$ 32$ Abs. 1 S. 1 KWG zu einer Erlaubnispflicht, wenn das Unternehmen die Finanzdienstleistungen für andere gewerbsmäßig oder in einem Umfang erbringt, der einen kaufmännischen Geschäftsbetrieb erfordert. Tatbestandliche Voraussetzung der Erlaubnispflicht ist somit eine Tätigkeit, die entweder gewerbsmäßig erfolgt oder in einem Umfang, der einen in kaufmännischer Weise eingerichteten Geschäftsbetrieb erfordert.

Hierbei kommt es auf die Rechtsform des Unternehmens nicht an, sodass für eine Erlaubnispflicht natürliche wie juristische Personen sowie Personengesellschaften in Betracht kommen. ${ }^{24}$

Finanzdienstleistungen werden, auch wenn der Umfang dieser Geschäfte objektiv keinen in kaufmännischer Weise eingerichteten Geschäftsbetrieb erfordert, gewerbsmäßig betrieben, wenn der Betrieb auf eine gewisse Dauer angelegt ist und der Betreiber ihn mit der Absicht der Gewinnerzielung verfolgt. ${ }^{25}$ Unter letzteres fällt auch die Absicht der Vermeidung von Verlusten. ${ }^{26}$ Auf Dauer ist die Tätigkeit angelegt, wenn sie nachhaltig und planmäßig erfolgen soll, i.d.R. also mit der erkennbaren Absicht der Wiederholung bzw. Fortsetzung, ohne dass hierzu zwingend ein ununterbrochener Geschäftsbetrieb erforderlich wäre.

Dem Erfordernis eines in kaufmännischer Weise eingerichteten Geschäftsbetriebs kommt seit der Sechsten KWG-Novelle ${ }^{27}$ nur noch eine untergeordnete Bedeutung zu, weil das Merkmal der Gewerbsmäßigkeit in der Regel bereits

24 BT-Drucks. 19/26925, S. 74.

25 BGH NJW-RR 2011, 350.

$26 \mathrm{Vgl}$. BaFin, Merkblatt: Hinweise zum Tatbestand des Kryptoverwahrgeschäfts vom 02.03.2020, abrufbar unter https://www.bafin.de/SharedDocs/Veroeffentlichungen/DE/ Merkblatt/mb_200302_kryptoverwahrgeschaeft.html;jsessionid=D22E49AA261A3B8 1B866485A67F82CDD.1_cid501?nn=9450978\#doc13710900bodyText6.

27 Binder, in: ders./Glos/Riepe (Hrsg.), Handbuch Bankaufsichtsrecht (2. Aufl. 2020), \18 Rn.63; Boos/Fischer/Schulte-Mattler/Fischer/Müller, KWG (5. Aufl. 2016), \32 Rn. 8; zur Gesetzgebungsgeschichte vgl. Schwennicke, Vergabe privater Darlehen und Erlaubnispflicht nach dem KWG, WM 2010, 542, $543 \mathrm{ff}$. 
zuvor greift. ${ }^{28}$ Nur wenn die Gewinnerzielungsabsicht oder Entgeltlichkeit zu verneinen sind, ist im Einzelfall zu prüfen, ob die betriebenen Bankgeschäfte einen in kaufmännischer Weise eingerichteten Geschäftsbetrieb erfordern. Solche Fälle sind in Zusammenhang mit der Kryptowertpapierregisterführung nur schwer denkbar. Jedenfalls ist es unerheblich, ob tatsächlich ein in kaufmännischer Weise eingerichteter Geschäftsbetrieb geführt wird. ${ }^{29}$ Maßgebend ist allein, ob für den Finanzdienstleistungsbetrieb nach der bankwirtschaftlichen Verkehrsauffassung (d.h. aus der Perspektive eines ordentlichen Kaufmanns) die Einrichtung eines solchen Betriebs objektiv erforderlich ist. ${ }^{30}$ Dies ist im Einzelfall zu bestimmen und kann sich beim gleichzeitigen Betreiben mehrerer Bank-/Finanzdienstleistungsgeschäfte auch bei einem vergleichsweise geringen Umfang ergeben. ${ }^{31}$

Unter den Erlaubnisvorbehalt nach $₫ 32$ Abs. $1 \mathrm{KWG}$ fällt das Geschäft nur, wenn es (auch) im Inland betrieben wird. Das Geschäft wird im Inland betrieben, wenn das Unternehmen seinen Sitz im Inland hat, auch wenn es das Geschäft gezielt aus dem Inland heraus nur mit Nicht-Gebietsansässigen betreibt. ${ }^{32}$ Das Geschäft wird darüber hinaus auch im Inland betrieben, wenn das Unternehmen hier eine rechtlich unselbständige Zweigniederlassung errichtet oder eine andere physische Präsenz unterhält, von der aus es die Geschäfte und sei es auch nur gezielt mit Nicht-Gebietsansässigen - betreibt. ${ }^{33} \mathrm{Ob}$ der Registerführer seinen Sitz in Deutschland haben muss, entscheidet sich nach allgemeinen Grundsätzen (\$S $32,53,53 \mathrm{~b}$ KWG). Damit dürfte eine Zweigniederlassung ausreichen und zwar im Falle einer EU-Zweigniederlassung, wenn ihre vom Heimatstaat erteilte Lizenz auch das Depotgeschäft abdeckt, die als die weiterreichende Lizenz auch das Kryptoverwahr- und Kryptoregisterführungsgeschäft umfassen sollte.

\footnotetext{
${ }^{28}$ Vgl. Boos/Fischer/Schulte-Mattler/Fischer/Müller, KWG (5. Aufl. 2016), $\$ 32$ Rn. 8.

29 Vgl. BaFin, Merkblatt: Hinweise zum Tatbestand des Kryptoverwahrgeschäfts vom 02.03.2020 (siehe oben, Fn. 25).

30 Ebenroth/Boujong/Joost/Strohn/Kindler, HGB (4. Aufl. 2020), $\$ 1$ Rn. 47.

31 Vgl. BaFin, Merkblatt: Hinweise zum Tatbestand des Kryptoverwahrgeschäfts vom 02.03.2020 (siehe oben, Fn. 25).

32 Vgl. BaFin, Merkblatt: Hinweise zum Tatbestand des Kryptoverwahrgeschäfts vom 02.03.2020 (siehe oben, Fn. 25).

33 Vgl. BaFin, Merkblatt: Hinweise zum Tatbestand des Kryptoverwahrgeschäfts vom 02.03.2020 (siehe oben, Fn. 25).
} 
Der Erfüllungsaufwand der Verwaltung für die Bearbeitung der Erlaubnisanträge beträgt voraussichtlich:

\begin{tabular}{|c|c|c|c|c|c|c|}
\hline Gesetz & Paragraf & Inbalt & $\begin{array}{l}\text { Kom- } \\
\text { plexität }\end{array}$ & $\begin{array}{l}\text { Zeit } \\
\text { in Min. }\end{array}$ & $\begin{array}{l}\text { Fall- } \\
\text { zabl }\end{array}$ & $\begin{array}{l}\text { Erfüllungs- } \\
\text { aufwand } \\
\text { gesamt }\end{array}$ \\
\hline eWpG & $\$ 20$ Abs. 3 & $\begin{array}{l}\text { BaFin: Führung öffent- } \\
\text { licheer Liste über Krypto- } \\
\text { WP im Internet }\end{array}$ & einfach & 764 & 1 & $565,11 €$ \\
\hline KWG & $\begin{array}{l}₫ 32 \text { Abs. } 1 \\
\text { i.V.m. } \$ 1 \\
\text { Abs. } 1 \mathrm{a} \\
\text { S. } 2 \text { Nr. } 8\end{array}$ & $\begin{array}{l}\text { Bearbeitung Erlaubnis- } \\
\text { antrag (neu) für Krypto- } \\
\text { wertpapierregisterführer }\end{array}$ & hoch & 5365 & 15 & $122.804,85 €$ \\
\hline KWG & $\begin{array}{l}₫ 32 \text { Abs. } 1 \\
\text { i.V.m. } \$ 1 \\
\text { Abs. } 1 \mathrm{a} \\
\text { S. } 2 \text { Nr. } 8\end{array}$ & $\begin{array}{l}\text { Bearbeitung Erlaubnis- } \\
\text { antrag (Erweiterung) für } \\
\text { Kryptowertpapierregis- } \\
\text { terführer }\end{array}$ & hoch & 5365 & 15 & $122.804,85 €$ \\
\hline
\end{tabular}

Tabelle 1: Einmaliger Erfüllungsaufwand (Quelle: RegE, S. 39).

\section{Einschreiten bei Verstößen oder unerlaubten Geschäften}

Bei Verstößen gegen das KWG und Missständen kann die BaFin nach $\$ 6$ Abs. 3 KWG mit den Instrumenten des Verwaltungsrechts, also hoheitlichen Maßnahmen einschreiten. Die Vorschrift enthält eine Generalklausel für ordnungsrechtliche Eingriffe der Bundesanstalt gegenüber Instituten und deren Geschäftsleitern. Sie ist nicht nur auf informelle Maßnahmen angewiesen. Sie kann damit ihre Vorstellungen per Anordnung durchsetzen. Diese Regelung ermöglicht ein schnelleres und effizienteres Einschreiten. Der Umweg über die Infragestellung der fachlichen Eignung oder Zuverlässigkeit von Geschäftsleitern erübrigt sich daher in vielen Fällen. ${ }^{34}$

Sollte dies nicht ausreichen, kann die Erlaubnis aufgehoben werden. Zwar kann die Aufhebung der Erlaubnis grundsätzlich auf einen der in $\$ 35 \mathrm{Abs.} 2$ KWG genannten Gründe gestützt werden. Liegt ein solcher Aufhebungsgrund vor, steht die Entscheidung im pflichtgemäßen Ermessen der Aufsichtsbehörde. Eine Aufhebung der Erlaubnis kommt wegen eines Verstoßes gegen Aufsichtsrecht nach $\$ 35$ Abs. 2 Nr. 6 KWG nur in Betracht, wenn gegen Bestimmungen des KWG, des GwG, des WpHG oder hierauf gestützter Verordnungen oder Anordnungen verstoßen wurde. Verstöße gegen andere rechtliche Vorschriften und damit auch nicht gegen Bestimmungen des eWpG sind von Nr. 6 nicht erfasst. In $\ 35$ Abs. 2 Nr. 6 KWG wird nicht auf das eWpG Bezug genommen.

\footnotetext{
${ }^{34}$ Vgl. Boos/Fischer/Schulte-Mattler/Fischer/Müller, KWG (5. Aufl. 2016), $\$ 6$ Rn. 58.
} 
Hier handelt es sich um ein noch zu behebendes, aber nicht schwerwiegendes Redaktionsversehen. Denn nach der ausdrücklichen Regelung in $\$ 35$ Abs. 2 KWG könnte bei nachhaltigen Verstößen gegen das eWpG die Aufhebung auch nach den allgemeinen Regelungen der $\int \mathbb{S} 48$ und 49 VwVfG über die Rücknahme und den Widerruf eines begünstigenden Verwaltungsakts erfolgen.

Daneben enthält $₫ 37$ eine Eingriffsbefugnis der BaFin, wenn Finanzdienstleistungen ohne Erlaubnis erbracht werden. Danach kann sie u.a. die sofortige Einstellung des Geschäftsbetriebes anordnen und diese notfalls im Wege des Verwaltungszwangs (VwVG) durchsetzen. Mit der Bestimmung des $\$ 44 c$ KWG werden der BaFin eine Auskunfts- und Vorlagepflicht (Abs. 1), ein Prüfungsund Betretungsrecht (Abs.2) und eine Durchsuchungsbefugnis (Abs.3) sowie eine Duldungspflicht gegenüber den betroffenen Unternehmen eingeräumt, die keine Institute i.S. d. KWG sind. Danach kann die BaFin im Rahmen der Verfolgung unerlaubter Finanzdienstleistungen Auskünfte über die Geschäftsangelegenheiten und die Vorlage von Unterlagen bei demjenigen verlangen, der im Verdacht solcher Handlungen steht oder wenn über den Verdachtsgrad hinaus dieser Sachverhalt bereits feststeht.

\section{Strafbares Handeln ohne Erlaubnis}

Das Erbringen von Finanzdienstleistungen ohne die erforderliche Erlaubnis nach $₫ 32$ Abs. 1 S. $1 \mathrm{KWG}$ ist gem. $\ 54$ Abs. 1 Nr. 2 KWG strafbewehrt. Schutzgut der Strafnorm sind die Effektivität der Aufsicht und die Funktionsfähigkeit des Finanzwesens, das durch potentiell unzuverlässige Geschäftsleiter oder unzulänglich mit Eigenkapital ausgestattete Finanzintermediäre gefährdet wird. Jede Geschäftsaufnahme ohne die entsprechende Erlaubnis verwirklicht bei Erreichen des erlaubnispflichtigen Umfangs den objektiven Tatbestand der relevantesten Strafnorm des KWG. Der Strafrahmen erstreckt sich von einer Geldstrafe bis hin zu einer Freiheitsstrafe von bis zu fünf Jahren.

Unerheblich ist, ob das Geschäft erlaubnisfähig ist oder nachträglich erlaubt wird, denn für die Beurteilung der Tat ist stets die zum Zeitpunkt der Handlung bestehende Rechtslage maßgebend (vgl. \$8 StGB).

Der Versuch des Betreibens von Geschäften ohne die erforderliche Erlaubnis ist nicht strafbar (vgl. $\$ \int 23$ Abs. 1, 12 Abs. 1 und 2 StGB). Das bloße Anbieten oder Anpreisen solcher Geschäfte sowie organisatorische Vorbereitungen bleiben daher straflos.

\section{Verstöße gegen Bußgeldvorschriften}

\56 KWG beinhaltet diverse Bußgeldtatbestände, die auch für die Kyptowertpapierregisterführung gelten. Exemplarisch sei $\$ 56$ Abs.4h Nr. 4 KWG angeführt, wonach ordnungswidrig handelt, wer wenigstens leichtfertig bei dem Erlaubnisantrag nach $\ 32$ Abs. 1 S. 2 KWG unrichtige Angaben macht. Für die 
jeweiligen Tatbestände legt $\ 56$ Abs. 6 KWG einen Bußgeldrahmen fest. Die Geldbuße liegt dabei zwischen 100.000,00 EUR bis zu 5.000.000,00 EUR. Hier können für Verstöße gegen Vorschriften des KWG daher höhere Bußgeldrahmen bestehen als sie in $\$ 31 \mathrm{eWpG}$ vorgesehen sind.

Auch für Verstöße gegen geldwäscherechtliche Vorgaben können nach dem KWG und dem GWG wesentlich höhere Bußgelder gegenüber einer juristischen Person oder einer Personenvereinigung verhängt werden. Sofern es sich um nachhaltige Verstöße handelt, liegt der Bußgeldrahmen nach $\ 56$ Abs. $6 \mathrm{~b}$ KWG bei juristischen Personen oder Personenvereinigungen bei 10 Prozent des Gesamtumsatzes, den diese im Geschäftsjahr, das der Behördenentscheidung vorausgegangen ist, erwirtschaftet haben.

\section{Aufsicht über die ordnungsgemäße Führung von Kryptowertpapierregistern}

Die allgemeine Zuständigkeit der BaFin zur Aufsicht erstreckt sich gem. \11 Abs. 1 eWpG auf die Aufsicht zur Führung von Wertpapierregistern nach $\ 16$ eWpG. Das Gesetz sieht besondere funktionale und technische Anforderungen an die Ausgestaltung eines dezentralen, fälschungssicheren Aufzeichnungssystems vor. $\$ 11$ Abs. 2 eWpG regelt die Zuständigkeit der BaFin als Aufsichtsbehörde i.S.d. Gesetzes.

Der Erfüllungsaufwand der Verwaltung für die Prüfung der Einhaltung der Vorgaben an das Führen von Kryptowertpapier gegen $\$ \$ \$ 7,10,12,16$ bis 22 eWpG beträgt voraussichtlich:

\begin{tabular}{|c|c|c|c|c|c|c|}
\hline Gesetz & Paragraf & Inbalt & $\begin{array}{l}\text { Kom- } \\
\text { plexität }\end{array}$ & $\begin{array}{l}\text { Zeit } \\
\text { in Min. }\end{array}$ & $\begin{array}{l}\text { Fall- } \\
\text { zabl }\end{array}$ & $\begin{array}{l}\text { Erfüllungs- } \\
\text { aufwand } \\
\text { gesamt }\end{array}$ \\
\hline PrüfBV & $\$ 69 a$ & $\begin{array}{l}\text { Prüfung der Einhal- } \\
\text { tung der Vorgaben an } \\
\text { das Führen zentraler } \\
\text { Register }\left(\$ \int 7,10,12\right. \\
\text { und } 13 \text { eWpG) }\end{array}$ & hoch & 7675 & 20 & $234.241,00 €$ \\
\hline PrüfBV & $\$ 69 \mathrm{~b}$ & $\begin{array}{l}\text { Prüfung der Einhal- } \\
\text { tung der Vorgaben an } \\
\text { das Führen von Kryp- } \\
\text { to-WP-Registern ( } \mathbb{S} 7 \text {, } \\
\text { 10, } 16 \text { und } 22 \text { eWpG) }\end{array}$ & hoch & 7675 & 30 & $351.361,50 €$ \\
\hline
\end{tabular}

Tabelle 2: Erfüllungsaufwand der Verwaltung für die Prüfung der Einhaltung der Vorgaben (Quelle: RegE, S.39). 


\section{Funktionale und technische Anforderungen}

Das eWpG-E enthält in den $\int \mathbb{S} 16 \mathrm{ff}$. insoweit nur sehr rudimentäre Anforderungen und verlagert sämtliche Detailregelungen auf eine noch zu erlassende Verordnung nach $\$ 23 \mathrm{eWpG}$. Ein Entwurf dieser Verordnung nach Anforderungen an elektronische Wertpapiere (eWpRv) wurde am 05.08.2021 vorgelegt. ${ }^{35}$ Mit dieser Gesetzgebungstechnik kann das Bundesministerium der Justiz und für Verbraucherschutz und das Bundesministerium für Finanzen Vorschriften zur Registerführung im Fall von technischen Neuerungen rascher und flexibler anpassen.

\section{Bußgeldvorschriften}

Stellt sich heraus, dass es beim Betrieb des Kryptowertpapierregisters zu Zuwiderhandlungen kommt, kann die BaFin zum einen im Rahmen eines Verwaltungsverfahrens anordnen, das beanstandete Verhalten zu beenden. Dazu können auch Zwangsmittel eingesetzt werden. Zum anderen kann die BaFin im Wege eines Ordnungswidrigkeitenverfahrens eine Geldbuße verhängen. Verstöße gegen öffentlich-rechtliche Vorschriften des eWpG sind nach $\$ 31 \mathrm{eWpG}$ mit Geldbuße bedroht. Auf diese Weise soll der Bedeutung der sanktionsbewehrten Vorschriften für den Anlegerschutz und für den Schutz des geordneten Ablaufs kapitalmarktrechtlicher Transaktionen und intendierter Kapitalmarktintegrität angemessen Rechnung getragen werden. ${ }^{36}$

Der Erfüllungsaufwand der Verwaltung für die Überwachung und Verfolgung von Verstößen gegen $\$ \int 7,10,12,16$ bis 22 eWpG beträgt voraussichtlich:

\begin{tabular}{|c|c|c|c|c|c|c|}
\hline Gesetz & Paragraf & Inbalt & $\begin{array}{l}\text { Kom- } \\
\text { plexität }\end{array}$ & $\begin{array}{l}\text { Zeit } \\
\text { in Min. }\end{array}$ & $\begin{array}{l}\text { Fall- } \\
\text { zabl }\end{array}$ & $\begin{array}{l}\text { Erfüllungs- } \\
\text { aufwand } \\
\text { gesamt }\end{array}$ \\
\hline eWpG & $\begin{array}{l}₫ 31 \\
\text { Abs. } 2\end{array}$ & 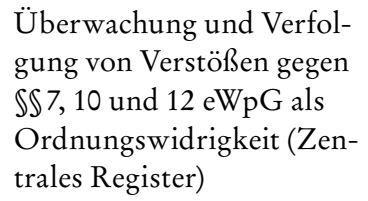 & hoch & 5785 & 20 & $176.558,20 €$ \\
\hline eWpG & $\begin{array}{l}₫ 31 \\
\text { Abs. } 3\end{array}$ & 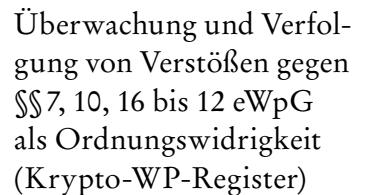 & hoch & 5785 & 30 & $264.837,50 €$ \\
\hline
\end{tabular}

Tabelle 3: Erfüllungsaufwand der Verwaltung für die Überwachung und Verfolgung von Verstößen (Quelle: RegE, S.39).

\footnotetext{
35 www.bmf.de.

36 BT-Drucks. 19/26925, S. 69.
} 


\section{a) Zielwirkungen}

Die Ahndung mittels Geldbußen zählt zu den schärfsten und effektivsten Maßnahmen des aufsichtsrechtlichen Eingriffsinstrumentariums, die die BaFin ergreifen kann, um mittelbar über die wertpapierrechtliche Normeinübung die Funktionsfähigkeit des Kapitalmarkts und damit mittelbar Anlegerschutz zu erreichen. Die Normanerkennung kapitalmarktrechtlicher Vorschriften im Interesse der wichtigen, übergeordneten Regelungsziele des Funktions- und Anlegerschutzes können in der Ahndungspraxis insbesondere durch die Verhängung selbständiger Verbandsordnungswidrigkeiten erreicht werden. Namentlich bei der Verletzung von Sondertatbeständen ist die sogenannte isolierte oder selbständige Verbandsgeldbuße, die im selbständigen Verfahren gem. \30 Abs. 1, Abs. 4 OWiG verhängt wird, bislang das vorrangige Instrument der Ahndung in der Aufsicht. Die Tradition einer isolierten Verbandssanktion mag im ersten Moment überraschen, weil sie gleich mit zwei herkömmlichen Prinzipien bricht. Zum einen, ist die Verhängung einer kumulativen Verbandsgeldbuße der gesetzliche Regelfall, die isolierte oder selbständige Verbandsgeldbuße der Ausnahmefall. Der zweite Bruch ist vom Ausgangspunkt individualstrafrechtlicher Verantwortung ein radikaler Perspektivwechsel: Nicht mehr das Einzelsubjekt, sondern der Verband ist als Täter in den Mittelpunkt der Verfolgung und Ahndung gerückt. Vom Ansatz her gilt aber insbesondere im Kriminalstrafrecht der Gedanke, dass nicht Kooperationen, sondern Individuen für das einstehen müssen, was sie getan oder unterlassen haben, gleichgültig ob sie für Unternehmen gehandelt haben oder nicht. Die mittlerweile gefestigte Übung, vorrangig isolierte Verbandssanktionen zu verhängen, ist jedoch weder hinsichtlich der verfolgten Schutzzwecke noch aus materiellen Gesichtspunkten insuffizient. Schließlich trägt die Beschränkung auf das selbständige Verfahren und die isolierte Ahndung des Verbandes auch im Interesse aller Beteiligten zu einer signifikanten Verfahrensbeschleunigung bei.

Die Ahndung von Verbänden kann neben der repressiven Funktion, im Besonderen sowohl spezial- als auch generalpräventive Wirkung entfalten. Durch die ahndende Geldbuße erhält der betroffene Verband einen Tadel, damit die rechtsverbindliche Vorschrift insgesamt nochmals ins Bewusstsein rückt. Die Ahndung des Verbandes geht damit in der Appellfunktion mittelbar über die Inanspruchnahme der handelnden oder unterlassenden Leitungsperson hinaus, weil sie sämtlichen Organen und verantwortlich handelnden Personen vor Augen führt, dass Verstöße gegen Verhaltensnormen des öffentlichen Kapitalmarktrechts für den Verband nachteilige Folgen haben.

Die Geldbuße zielt als Nebenwirkung darüber hinaus auch auf die Verbandsmitglieder ab. Diese sollen zu einer sorgfältigen Auswahl und Überwachung von Repräsentanten, insbesondere der vertretungsberechtigten Organe, angehalten werden. Das Risiko der Verwirklichung einer Schmälerung der Rendite aus 
dem der Kooperation zur Verfügung gestellten Kapital, lässt sich so zumindest abstrakt durch die Auswahl des Vertretungsberechtigten sowie des Aufsichtsorgans steuern. Verbände sind durch ihren - wechselnden - Mitgliederbestand, ihre Mitarbeiter und ihre Organe zwar ein künstliches, aber dennoch ein mit Leben gefülltes Gebilde, das jedenfalls für diese, mit der Geldbuße verfolgten Zielwirkungen empfänglich ist.

Spürbare Verbandsgeldbußen sind insofern geeignet, hinsichtlich der gesamten Kooperation eine hinlängliche präventive Wirkung zu entfalten, in dem sie diese zu weitergehenden Sicherungsmaßnahmen anhalten, damit es nicht erneut zu Pflichtverletzungen kommt. Verstöße gegen $\ 31$ Abs. 3 eWpG können zwar mit einer Geldbuße bis zu 100000 EUR geahndet werden. Unabhängig von den in $\$ 31 \mathrm{eWpG}$ normierten Bußgeldrahmen gelten daneben bereits die nach dem KWG und GWG geltenden höheren Sanktionsvorgaben. ${ }^{37}$

Erfahrungsgemäß führen Bußgelder dazu, dass vielfach die internen Arbeitsabläufe und Prozesse innerhalb des Verbandes verbessert oder umstrukturiert sowie weitergehende Compliancestrukturen geschaffen werden. Aus dem Blickwinkel der Spezialprävention, die ein zentrales Element der Ahndung auch im selbständigen Bußgeldverfahren darstellt, sind interne Maßnahmen, welche der Anerkennung sowie Internalisierung von kapitalmarktrechtlichen Normen dienen, nicht nur zu begrüßen, sondern anzustreben.

Verbandsinterne Anstrengungen und Maßnahmen zur Vermeidung künftiger Rückfallordnungswidrigkeiten werden bereits im laufenden Verfahren ausdrücklich als milderndes Anpassungskriterium anerkannt. Als solches sind sie verbindlich im Annex zu den WpHG-Bußgeldleitlinien aufgeführt. ${ }^{38}$ Unter dieser Prämisse können mitunter - einzelfallbezogen - Investitionen in Compliance- und organisatorische Sicherungsmaßnahmen bei der Zumessung der Verbandsgeldbuße mildernd herangezogen werden.

Die Verfolgung und Ahndung soll darüber hinaus aber auch andere generalpräventiv dazu anhalten können, die kapitalmarktrechtlichen Vorschriften einzuhalten und sorgfältig zu beachten. Nicht die Abschreckungs-, wohl aber die positive Generalprävention steht dann im Vordergrund. Aufgrund der abschreckenden Wirkung, die spürbare und hohe Geldbußen gleichwohl auch auf andere entfalten, können sie, Zuwiderhandlungen gegen die einschlägigen Verwaltungsnormen effektiv vorbeugen helfen.

37 Siehe oben unter II. 5.

$38 \mathrm{Vgl}$.https://www.bafin.de/SharedDocs/Downloads/DE/Leitfaden/WA/dl_aktualisie rung_bussgeldleitlinien_2018.pdf;jsessionid=4C3A7061B0BD1708AED093EBDFA025 94.2_cid502?__blob=publicationFile\&v=6, S.6; vgl. Baums/von Buttlar, Der Monitor im Unternehmensrecht, ZHR 184 (2020), $259 \mathrm{ff}$. 


\section{b) Bedingungen für eine Verbandssanktion}

Die Bedingungen für eine Verbandssanktion nach $\ 30$ OWiG liegen im Kapitalmarktrecht regelmäßig vor. Allgemeine Voraussetzung für die Festsetzung einer Geldbuße gegen den Verband ist insofern, dass ein vertretungsberechtigtes Organ einer juristischen Person oder ein Mitglied des in $\$ 30$ Abs. 1 Nr. 2Nr. 5 OWiG genannten Personenkreises als sog. Anknüpfungs- oder Bezugstat eine Ordnungswidrigkeit - oder Straftat - begangen hat, durch die Pflichten verletzt worden sind, die nach der Normenadressierung oder wegen der Betriebsbezogenheit die juristische Person treffen.

Als Repräsentant kommt zunächst das vertretungsberechtigte Organ, im Falle des kapitalmarktrechtlichen Bußgeldverfahrens namentlich der Vorstand der Aktiengesellschaft in Betracht. Im Sinne des $\$ 30$ OWiG kann der Verband jedoch nicht nur durch das vertretungsberechtige Organ, sondern generell seitens seiner verantwortlich handelnden Leitungspersonen repräsentiert werden. Leitungspersonen repräsentieren das Verbandssystem aber nur dann, wenn zwischen den in Frage kommenden Taten und ihren Pflichten ein funktionaler Zusammenhang besteht. Im Falle von Sonderpflichten ist danach zunächst erforderlich, dass sie über $\$ 9$ Abs. 2 OWiG in die Normadressateneigenschaft inkorporiert werden. Die Rolle des Normadressaten kann anstelle des zur Vertretung berechtigten Organs aber nicht schlechthin jeder Mitarbeiter oder sonstige Vertreter einnehmen, sondern nur derjenige, dem die einschlägige Verantwortung zur selbständigen und eigenverantwortlichen Pflichtenwahrnehmung entweder als Funktionsträger oder qua ausdrücklicher Delegation übertragen worden ist. Dies setzt eine autarke Handlungsautonomie, eine weisungsunabhängige Pflichtenerfüllung sowie die allgemeinen Kompetenzen, die eine Wahl zwischen verschiedenen sachlichen, personellen und organisatorischen Alternativen ermöglichen, voraus. ${ }^{39}$ Wichtige kapitalmarktrechtliche Sondervorschriften werden der Vorstand oder sonstige Leitungspersonen in der Praxis nicht vollends auf funktionale Hierarchieebenen zur selbständigen und eigenverantwortlichen Wahrnehmung verlagern können, die nicht mindestens noch zum Täterkreis des $₫ 30$ Abs. 1 Nr. 5 OWiG zählen. Die Erfüllung der wertpapierrechtlichen Gebote kann daher nur auf solche Mitarbeiter delegiert werden, die wegen ihrer Kompetenz und Sachkunde in der Lage sind, diese ordnungsgemäß zu erfüllen. Hingegen dürfte es einen Organisationsmangel begründen, wenn die Verantwortung für wichtige Sondergebote erkennbar auf eine zu tiefe Hierarchieebene delegiert wird.

Im Einzelfall kann die Normadressierung daher neben dem vertretungsberechtigten Organ insbesondere um verantwortlich leitende Abteilungs-, Bereichs- oder Filialleiter, der Leiter der Rechtsabteilung, des Risikomanagements

39 Vgl. von Buttlar/Diesinger, Vorstände haften für schlechte Compliance, BaFinJournal $4 / 2021,26 f$. 
und der internen Revision erweitert sein. Nach der lex lata sind Zuwiderhandlungen dieser Leitungspersonen ohne Besonderheiten durch eine selbständige Verbandsgeldbuße ahndbar. Es handelt sich bei der Verletzung der im eWpG funktionalen und technischen Anforderungen an die Ausgestaltung eines dezentralen, fälschungssicheren Aufzeichnungssystems um solche betriebsbezogenen Sonderpflichten, die dem Verband im Sinne des $\$ 30$ OWiG obliegen. Im Falle der Sonder- oder Pflichtdelikte ist die Betriebs- oder Unternehmensbezogenheit praktisch immer zu bejahen. Bezugstaten des Vorstands oder von sonstigen Leitungspersonen können im Kapitalmarktrecht damit weitestgehend gegenüber dem Verband geahndet werden. Das gilt auch für die Aufsichtspflichtverletzung nach $\$ 130 \mathrm{OWiG}$, die auch in der Bußgeldpraxis als wichtige Anknüpfungstat vorkommt.

\section{c) Tatbestandsstrukturen}

Hinsichtlich der technischen und funktionalen Anforderungen an die Kryptowertpapierregisterführung enthält $\ 31 \mathrm{eWpG}$ drei unterschiedliche Unrechtskategorien, die teilweise auch leichtfertig verwirklicht werden können:

\begin{tabular}{|c|c|c|}
\hline Norm in $₫ 31 \mathrm{eWp} G$ & $\begin{array}{l}\text { Verbaltens-und } \\
\text { Schuldform }\end{array}$ & Unrechtskategorie \\
\hline Abs. 1 Nr. 1 & Vorsatz erforderlich & $\begin{array}{l}\text { Verstoß gegen gesetzliche } \\
\text { Pflichten }\end{array}$ \\
\hline Abs. $1 \mathrm{Nr} .2$ & Vorsatz & Verwaltungsaktsungehorsam \\
\hline $\begin{array}{l}\text { Abs. } 2 \text { Nr. } 5-8, \text { Nr. } 11- \\
12,19\end{array}$ & Fahrlässigkeit ausreichend & $\begin{array}{l}\text { Verstoß gegen gesetzliche } \\
\text { Pflichten }\end{array}$ \\
\hline $\begin{array}{l}\text { Abs. } 2 \text { Nr. 1-5, Nr. 9-10, } \\
\text { Nr. 13-14 }\end{array}$ & Fahrlässigkeit ausreichend & $\begin{array}{l}\text { Verstoß gegen organisatori- } \\
\text { sche Anforderungen }\end{array}$ \\
\hline
\end{tabular}

Tabelle 4: Unrechtskategorien in $\$ 31 \mathrm{eWpG}$.

Ein Verstoß gegen gesetzliche Pflichten liegt beispielsweise vor, wenn der Kryptowertpapierregisterführer entgegen $\$ 10$ Abs. $1 \mathrm{eWpG}$ eine Einsicht nicht oder nicht rechtzeitig gewährt (vgl. \31 Abs. 2 Nr. 5 eWpG).

Die Tatbestände mit Verwaltungsaktsungehorsam dienen in der Regel dazu, das Aufsichtsrecht durchzusetzen. Deshalb sind Anordnungen gegen vollziehbare Anordnung der BaFin nach $\$ 21$ Abs. 2 S. 2 eWpG bußgeldbewehrt (vgl. \31 Abs. 1 Nr. 2 eWpG).

Die rechtsstrukturell interessanteste und qualitativ gewichtigste Fallgruppe ist der dritte Unrechtstypus: bei Verstößen gegen organisatorische Anforderungen geht es nicht um individuelles Fehlverhalten, sondern um funktionale und mehr noch IT-technische Unzulänglichkeiten des Kryptowertpapierregister- 
führers. So sanktioniert $₫ 31$ Abs. 2 Nr.13 eWpG bei noch einiger Unschärfe das nicht richtige Führen eines Wertpapierregisters. Die erforderliche Sorgfalt hängt maßgeblich von den zur Kryptowertpapierregisterführung beachtenden Vorschriften ab. Ob hier ein Verstoß vorliegt, wird an der noch nach $\$ 23$ eWpG zu erlassenden Verordnung zu messen sein. Diese Vorschriften werden ausschließlich für die konkreten Normadressaten besondere Anforderungen an sorgfaltsgemäßes Verhalten aufstellen. Sorgfaltspflichten werden sich auch aus ungeschriebenen Verkehrsgepflogenheiten, im Besonderen aus Verwaltungsrichtlinien der BaFin ableiten lassen, die nichtgesetzlicher Natur sind. Zu denken ist hier etwa an konkretisierende Verordnungen oder sonstige Verlautbarungen sowie Rundschreiben der BaFin.

\section{Elektronische Wertpapiere und Marktaufsicht}

Elektronische Wertpapiere unterliegen dem Anwendungsbereich des Wertpapierhandelsgesetzes (WpHG), da der Wertpapierbegriff des $\$ 2$ Abs. 1 WpHG keine körperliche Verbriefung voraussetzt. Das (nationale und europäische) Aufsichtsrecht kennt dieses Urkundserfordernis - im Gegensatz zum DepotG bis zum Inkrafttreten des eWpG - nach h. M. nicht. So definiert $\$ 2$ Abs. 1 WpHG in Umsetzung der MiFID II Wertpapiere explizit als „alle Gattungen von übertragbaren Wertpapieren [...], die ihrer Art nach auf den Finanzmärkten handelbar sind“, „auch wenn keine Urkunden über sie ausgestellt sind“. Auch die EU-Prospektverordnung nimmt für die Wertpapier-Definition auf den Begriff der MiFID II Bezug.

Damit finden die Regeln des Sekundärmarkts, also das Recht des Wertpapierhandels, auf die elektronischen Wertpapiere Anwendung. Das gilt auch für die Vorschriften der Marktmissbrauchsverordnung (EU Nr. 596/2014). Sofern die elektronischen Wertpapiere i.S.d. eWpG als Wertpapiere im aufsichtsrechtlichen Sinne angesehen und in der europäischen Union öffentlich angeboten werden, unterfallen sie grundsätzlich nach Art. 3 Abs. 1 Prospekt-VO der Prospektpflicht. Die Änderungen im WpPG beschränken sich auf Regelungen zum ausschließlich national geregelten Informationsblatt. So darf das WIB nach $₫ 4$ Abs. 3 WpPG-E anstelle der regulären drei DIN-A4 Seiten nun vier umfassen. Aus Gründen des Anlegerschutzes sind weitere Angaben zur technischen Ausgestaltung des Wertpapiers, der zugrundeliegenden Technologien sowie zur Handel- und Übertragbarkeit erforderlich (vgl. \4 Abs. 3a Nr. 2 WpPG).

Der Gesetzentwurf trifft keine Aussage darüber, welche Rechtsnatur Kryptowerte außerhalb des Wertpapierbereichs haben und wie sie übertragen werden sollen. Die in einem noch sehr frühen Entwicklungsstadium befindliche Entwurf einer „Markets in Crypto-assets“-Verordnung (MiCAR) ausweislich ihres Art. 2 Abs. 2 lit. a) gilt gerade nicht für Finanzinstrumente i.S.d. MiFID II und 
soll, gleich einem Auffangregelwerk, diejenigen „Kryptowerte“ erfassen, die nicht anderweitig geregelt sind oder werden. ${ }^{40}$

\section{Ausblick}

Nach einer Studie aus den USA wird eine vollständige Dematerialisierung von Wertpapieren zu einem kostengünstigeren, effizienteren, sichereren und wettbewerbsfähigeren Marktplatz beitragen. ${ }^{41}$ Deutschland verfolgt in diesem Zusammenhang einen schrittweisen und sanften Ansatz. Die Strategie Deutschlands hinter dem hier diskutierten Gesetzesentwurf ist es, die Vorteile elektronischer Wertpapiere mit geringem Transformationsrisiko zu ermöglichen und nötige Änderungen möglichst reibungslos in das bestehende Zivilund Aufsichtsrecht einzufügen.

Der Gesetzgeber sorgt mit den geplanten Änderungen für einen kräftigen digitalen Schub in der Rechtsentwicklung. Klares Ziel sollte es jedoch sein, dass die Regelungen als Vorlage für weitere Wertpapiere, insbesondere auch für elektronische Aktien, dienen. Nur so wird sich Deutschland als attraktiver Standort für die Blockchain-Technologie weiter behaupten können. Während einigen der Vorschlag nicht ehrgeizig genug ist, da es sich nicht um eine allgemeine Dematerialisierung von Vermögenswerten geht, halten andere den Gesetzesvorschlag für das Beste, was unter den gegebenen Umständen möglich ist. Mit diesem Gesetzentwurf bricht das deutsche Rechtssystem jedenfalls endgültig in das digitale Zeitalter auf.

40 Europäische Kommission, Vorschlag für eine Verordnung des Europäischen Parlaments und des Rates on Markets in Crypto-assets, and amending Directive (EU) 2019/1937, 24.9.2020, COM(2020) 593 final; vgl. Siadat, Markets in Crypto Assets Regulation - erster Einblick mit Schwerpunktsetzung auf Finanzinstrumente, RdF 2021, $12 \mathrm{f}$.

41 DTCC, Strengthening the U.S. Financial Markets: A Proposal to Fully Dematerialize Physical Securities, Eliminating the Costs and Risks They Incur (2012), abrufbar unter https://www.dtcc.com/ /media/Files/Downloads/WhitePapers/Dematerialize_Securities_ July_2012.pdf. 

Teil 3

Ausblick 



\section{$\$ 8$ \\ Elektronische Geschäftsanteile}

Florian Möslein

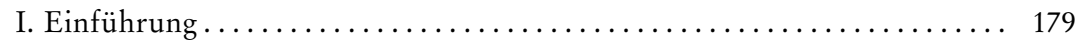

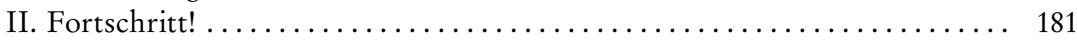

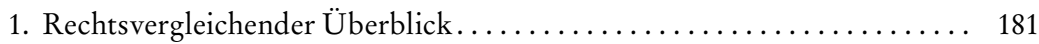

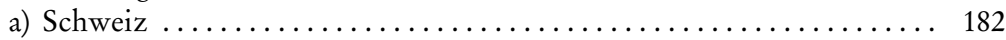

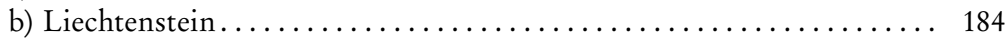

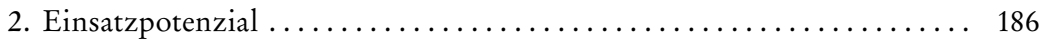

a) Gesellschafteridentifikation ....................... 187

b) Durchsetzung von Gesellschafterrechten und -pflichten ......... 188

3. Reformperspektiven ............................. 189

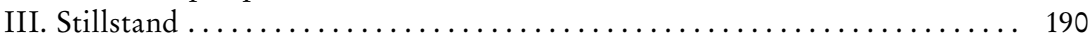

1. Selbstbeschränkung des eWpG ..................... 190

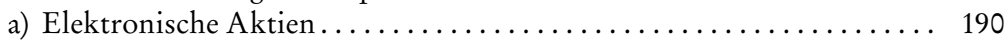

b) Elektronische Geschäftsanteile . .................... 191

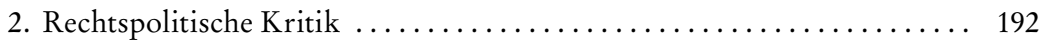

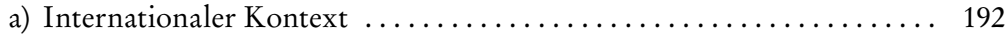

b) Gesellschaftsrechtliche Auswirkungen . ................. 193

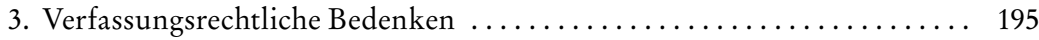

IV. Rückschritt? . . . . . . . . . . . . . . . . . . . . . . . . . . . . . . . 197

1. Praxis elektronischer Gesellschaftsanteile, insbesondere: Equity Token . 197

2. Zulässigkeit im geltenden Recht ....................... 199

a) Virtuelle Gesellschaftsanteile...................... 199

b) Unmittelbare Beteiligung, insbesondere durch Blockchain Listed

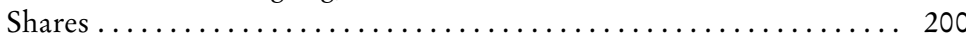

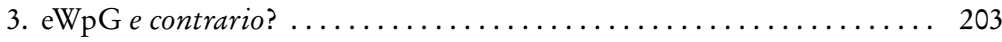

V. Zusammenfassung in Thesen ......................... 204

\section{Einführung}

Das deutsche Wertpapierrecht erlebt einen epochalen Umbruch: Wertpapiere können seit kurzem begeben werden, ohne dass es der Ausstellung einer Urkunde bedarf. Eröffnet wird diese Möglichkeit durch $\$ 2$ Abs. 1 des Gesetzes über elektronische Wertpapiere (eWpG). Dieses Gesetz wurde Anfang Mai 2021 vom Deutschen Bundestag verabschiedet und ist am 10. Juni 2021, dem 
Tag nach der Verkündung im Gesetzblatt, in Kraft getreten. ${ }^{1}$ Wertpapiere sollen nach der genannten Vorschrift auch elektronisch begeben werden können, und zwar dadurch, „dass der Emittent an Stelle der Ausstellung einer Wertpapierurkunde eine Eintragung in ein elektronisches Wertpapierregister bewirkt“. Die Möglichkeit der elektronischen Begebung soll nachweislich der Begründung des Referentenentwurfs dem Bedürfnis der Praxis Rechnung tragen, „eine Unternehmensfinanzierung auch durch Wertpapiere zu ermöglichen, die elektronisch und ggf. mittels der Blockchain-Technologie begeben werden“. ${ }^{2}$ Angesichts der Betonung des Zusammenspiels von Unternehmensfinanzierung und Wertpapierbegebung scheint das neue Gesetz darauf abzuzielen, die Finanzierung von Unternehmen zu erleichtern.

Während sich unternehmerischer Kapitalbedarf sowohl mit Eigen- als auch mit Fremdkapital decken lässt und Unternehmensfinanzierung daher stets zum Ziel hat, deren Relation unter Effizienzkriterien zu optimieren, ${ }^{3}$ regelt das eWpG indessen lediglich einen Teilbereich der Unternehmensfinanzierung: Weil sich sein Anwendungsbereich gem. $\$ 1$ auf Schuldverschreibungen auf den Inhaber beschränkt, betrifft es grundsätzlich nur Fremd-, nicht aber Eigenkapitalinstrumente. Elektronische Gesellschaftsanteile zählen, selbst wenn sie sich als Wertpapiere qualifizieren, nicht zu den elektronischen Wertpapieren, die das eWpG regelt. Der Gesetzestitel verspricht insofern mehr, als das Gesetz halten kann. Infolge der Beschränkung des $\$ 1 \mathrm{eWpG}$ handelt es sich in Wahrheit lediglich um ein Gesetz über elektronische Inhaberschuldverschreibungen.

Der nachfolgende Beitrag verbindet den Ausblick auf das mögliche Potenzial elektronischer Gesellschaftsanteile mit Kritik an jener schwerwiegenden, systematisch nicht stimmigen Beschränkung des $\$ 1 \mathrm{eWpG}$. Zunächst skizziert er das innovative Potenzial elektronischer und insbesondere tokenisierter Gesellschaftsanteile (unter II.). Rechtsvergleichend erweist sich der deutsche Regelungsansatz als singulärer Sonderweg, der effiziente Einsatzmöglichkeiten elektronischer Gesellschaftsanteile verbaut und selbst vom Gesetzgeber nur als Zwischenlösung angesehen wird. In einem zweiten Schritt versucht der Beitrag, die Motive dieser zumindest vorläufigen gesetzgeberischen Selbstbeschränkung zu ergründen (unter III.). Er stellt diese zugleich kritisch auf den Prüfstand

1 BGBl. I 2021, S. 1423.

2 Abrufbar unter https://www.bmjv.de/SharedDocs/Gesetzgebungsverfahren/Doku mente/RefE_Einfuehrung_elektr_Wertpapiere.html;jsessionid=87F7BB5F245A2D2087866 A71995445B8.2_cid297?nn=6712350, im Folgenden: RefE (alle Links in diesem Beitrag wurden zuletzt am 29.07.2021 abgerufen), dort S.1.

3 Grundlegend: Modigliani/Miller, The Cost of Capital, Corporation Finance and the Theory of Investment, American Economic Review 48 (1958), 261; einführend zum finanzierungstheoretischen Hintergrund dieser sog. Irrelevanztheorie sowie zu konkurrierenden (Trade-Off-)Theorien: Rudolph, Unternehmensfinanzierung und Kapitalmarkt (2006), S. 93-116; Schierenbeck/Wöhle, Grundzüge der Betriebswirtschaftslehre (19. Aufl. 2016), S. 567-580. 
und unterzieht sie einer verfassungsrechtlichen Wertung. Im dritten und letzten Schritt zeigt sich schließlich, dass der zögerliche Reformschritt die Gefahr rückschrittlicher Pfadabhängigkeiten birgt (unter IV.). Soweit die unternehmerische Praxis bereits von elektronischen Anteilen Gebrauch macht, stellen sich nämlich bislang ungeklärte Rechtsfragen, zu deren Beantwortung das eWpG beitragen kann - allerdings als argumentum e contrario, das die rechtliche Zulässigkeit solcher Anteile nochmals stärker in Frage stellt. Statt nach dem Vorbild ausländischer Regelungsmodelle Fortschritt zu ermöglichen, bedeutet das deutsche eWpG für elektronische Gesellschaftsanteile daher jedenfalls Stillstand, möglicherweise aber sogar einen signifikanten Rückschritt.

\section{Fortschritt!}

Elektronische Gesellschaftsanteile sind Ausdruck technischen Fortschritts und versprechen erhebliche ökonomische Effizienzvorteile. Insbesondere die Tokenisierung von Gesellschaftsanteilen auf Grundlage der Blockchain- bzw. Distributed-Ledger-Technologie eröffnet großes praktisches Anwendungspotenzial, wirft allerdings zugleich neben wertpapier- auch grundlegende gesellschaftsrechtliche Fragestellungen auf. Diese Grundfragen eines BlockchainKapitalgesellschaftsrechts sind insbesondere mit Blick auf Aktiengesellschaften und Gesellschaften mit beschränkter Haftung zwar bereits Gegenstand des rechtswissenschaftlichen Diskurses. ${ }^{4}$ Sie haben den deutschen Gesetzgeber aber vorerst davon abgehalten, Gesellschaftsanteile in den Anwendungsbereich des eWpG einzubeziehen. Immerhin wird eine spätere Öffnung für elektronische Aktien in der Entwurfsbegründung bereits in Aussicht gestellt (dazu näher unter 3.), so dass Hoffnung besteht, dass sich das praktische Einsatzpotenzial elektronischer Gesellschaftsanteile (hierzu unter 2.) nach dem Vorbild ausländischer Regelungsmodelle (unter 1.) zumindest in der Zukunft, vielleicht bereits in der kommenden Legislaturperiode, auch in Deutschland wird realisieren lassen.

\section{Rechtsvergleichender Überblick}

Die Verabschiedung des eWpG ist Teil der Blockchain-Strategie der deutschen Bundesregierung. ${ }^{5}$ Es ist daher eine - keineswegs die einzige - Ausprägung der gegenwärtigen Entwicklung hin zu einer breitflächigeren Krypto-Regulie-

${ }^{4}$ S. etwa Möslein/Omlor/Urbach, Grundfragen eines Blockchain-Kapitalgesellschaftsrechts, ZIP 2020, 2149.

5 Bundesregierung, Blockchain-Strategie. Wir stellen die Weichen für die Token-Ökonomie, 19.9.2019, BT-Drs. 19/13433, bes. S.6f.; vgl. ferner das gemeinsame Eckpunktepapier des Bundesministeriums der Finanzen und des Bundesministeriums der Justiz und für Verbraucherschutz für die regulatorische Behandlung von elektronischen Wertpapieren und 
rung. ${ }^{6}$ Eine solche Tendenz lässt sich derzeit nicht nur in Deutschland, sondern auch in zahlreichen Nachbarrechtsordnungen beobachten (und beschäftigt zudem auch den europäischen Regelgeber intensiv). ${ }^{7}$ Dabei zeichnet sich bereits ein veritabler Wettbewerb der Regelgeber ab: Während in den USA vor allem der Bundesstaat Wyoming als führend gilt und in Anspielung auf die führende gesellschaftsrechtliche Jurisdiktion als „Delaware of digital asset law“ bezeichnet wird, ${ }^{8}$ treten innerhalb Europas vor allem die Schweiz und Liechtenstein mit vielbeachteten neueren Krypto-Gesetzen hervor. Daneben sind auch die Gesetzgeber beispielsweise in Malta, Frankreich, Großbritannien und Italien nicht untätig geblieben, haben aber bislang eher punktuelle Regelungen erlassen bzw. Regelungsvorschläge unterbreitet. ${ }^{9}$

\section{a) Schweiz}

In der Schweiz hat die „Aktien-Revolution“ bereits stattgefunden: Seit 1. Februar 2021 um 00:00:01 Uhr sind die ersten tokenisierten Aktien als solche auf einer digitalen Aktienplattform erfasst. ${ }^{10}$ Exakt zu diesem Zeitpunkt ist das neue „Bundesgesetz zur Anpassung des Bundesrechts an Entwicklungen der Technik verteilter elektronischer Register" in Kraft getreten. ${ }^{11}$ Mit dem

Krypto-Token vom 7.03.2019, abrufbar unter https://www.bundesfinanzministerium.de/ Content/DE/Gesetzestexte/Gesetze_Gesetzesvorhaben/Abteilungen/Abteilung_VII/19_ Legislaturperiode/2019-03-07-Eckpunktepapier-Wertpapiere-Krypto-Token/2019-03-07Eckpunktepapier-regulatorische-Behandlung-elektronische-Wertpapiere-Krypto-Token. pdf?__blob=publicationFile\&v=3.

${ }^{6}$ Breiterer Überblick besonders auch zu einschlägigen finanzaufsichtsrechtlichen Regelungen ( $\mathbb{S} 1$ Abs. 1a S. 2 Nr. 6, 32 Abs. 1 S. 1 KWG) bei Möslein, in: Schwintowski, Bankrecht (6. Aufl. 2021), Kapitel 12, Rn. 14ff.; noch übergreifender, weit über den Finanzsektor hinaus: Denga, Die Regulierung der Blockchain-Infrastruktur, JZ 2021, 227.

7 Näher zur neueren europäischen Rechtsentwicklung Möslein/Omlor, in: dies. (Hrsg.), FinTech-Handbuch (2. Aufl. 2021), \$1 Rn. 14-47.

8 Long, What Do Wyoming's 13 New Blockchain Laws Mean?, Forbes.Com v. 4.3.2019, abrufbar unter https://www.forbes.com/sites/caitlinlong/2019/03/04/what-do-wyomingsnew-blockchain-laws-mean/\#6b0672635fde; näher zum jüngsten einschlägigen Gesetz, dem „Decentralized Autonomous Organization Supplement“, das DAOs als Gesellschaften mit beschränkter Haftung anerkennt: Mienert, Wyomings DAO-Gesetz - Blaupause für die Regulierung Blockchain-basierter dezentraler autonomer Organisationen?, RDi 2021, 384, $338-390$.

9 Kurzer Überblick bei Möslein, Smart Contracts im Zivil- und Handelsrecht, ZHR 183 (2019), 254, 255-257, m. w. N.; ausführlicher Omlor, Digitales Eigentum an Blockchain-Token - rechtsvergleichende Entwicklungslinien, ZVglRWiss 119 (2020), 41.

${ }^{10}$ Es handelt sich um die Aktien der MME Compliance AG; ein Video dieses „historischen Moments" ist unter https://youtu.be/-baJ1SMkKLI abrufbar; vgl. ferner die Pressemitteilung unter https://www.daura.ch/medienmitteilung-20210201-dlt-gesetzgebung/ („Die Aktien-Revolution im Schweizer Gesetz - daura machts vor").

${ }^{11}$ Dazu Zellweger-Gutknecht/Monnerat, Internationaler Kontext: schweizerisches Registerwertrecht, $\mathbb{\$} 1$, in diesem Band; Weber, Neue Blockchain-Gesetzgebung in der Schweiz, RDi 2021, 186; s. außerdem die Beiträge in ders./Kuhn (Hrsg.), Entwicklungen im Schweizer Blockchain-Recht (2021). 
breitflächigen Ziel, eine sichere rechtliche Basis für die Übertragung bzw. den Handel von Rechten mittels der Distributed-Ledger-Technologie (DLT) oder auch anderer, heute noch unbekannter Technologien zu schaffen, sofern diese eine ausreichend manipulationsresistente Datenverarbeitung gewährleisten, ${ }^{12}$ sieht es als Rahmengesetz die Anpassung einer Reihe von Bundesgesetzen vor. Eine Schlüsselrolle spielt dabei das neu geschaffene, so genannte Registerwertrecht, das in Art. 973d-973i OR geregelt ist. Entgegen seiner Bezeichnung ist das Registerwertrecht selbst kein Recht, sondern ein gesetzlich qualifiziertes Informationsmedium, das insofern einem Wertpapier ähnelt. ${ }^{13}$ Auf Grundlage einer Registrierungsvereinbarung verknüpft es Recht und Eintragung (Registrierung) in einem Wertrechteregister derart, dass das Recht nur noch über dieses Wertrechteregister geltend gemacht und auf andere übertragen werden kann (Art. 973d Abs. 1 Ziff. 2 OR). Es handelt sich mithin um „ein vom Privatrechtsverkehr entwickeltes Informationsmedium“, das „mit spezifischen Privilegien betreffend Transport, Legitimation und Verkehrsschutz ausgestattet“ ist und so „die Umlauffähigkeit der mit ihm dargestellten Rechte wesentlich erhöht“ ${ }^{14}$ Als registerwertrechtsfähig gelten laut Gesetzesbegründung grundsätzlich alle Rechte, die „in Wertpapieren verbrieft werden können“. 15

Im diametralen Gegensatz zum beschränkten Regelungszuschnitt des deutschen eWpG lassen sich daher auch - zumindest bestimmte - Gesellschaftsanteile als Registerwertrecht abbilden, nämlich insbesondere Aktien und Partizipationsscheine von Aktien- und Kommanditaktiengesellschaften, sofern die Statuten es zulassen (Art.622, 656a Abs. 2 und 764 Abs. 2 OR), nicht jedoch $\mathrm{GmbH}$ - oder Genossenschaftsanteile, die nämlich auch nicht als Wertpapier verbrieft werden können (Art. 784 Abs. 1 und 849 Abs. 1 ZGB). ${ }^{16}$ Die betreffenden Aktien können als Inhaber- oder auch als Namensaktie ausgestaltet sein. ${ }^{17}$ Selbst eine Vinkulierung nicht börsennotierter Aktien (Art.685 a ff. OR) steht einer Registrierung in einem Wertrechteregister nicht entgegen, sofern die Gesellschaft sicherstellt, dass die Übertragung auf dem Register vor

12 Bundesrat, Botschaft zum Bundesgesetz zur Anpassung des Bundesrechts an Entwicklungen der Technik verteilter elektronischer Register v. 27. November 2019, BBl. (Schweiz) 2020, S. 233, 234.

13 Kritisch zur Bezeichnung Zellweger-Gutknecht/Monnerat, Internationaler Kontext: schweizerisches Registerwertrecht, $\mathbb{\mathbb { 1 }} 1$, in diesem Band.

14 S. nochmals Zellweger-Gutknecht/Monnerat, Internationaler Kontext: schweizerisches Registerwertrecht, $\mathbb{1} 1$, in diesem Band.

15 Botschaft (Fn. 12), S. 276.

16 Näher von der Crone/Baumgartner, Digitalisierung des Aktienrechts - Die Ausgabe von Aktien als Registerwertrechte, SZW 2020, 351, 360; Kubn, in: Weber/ders. (Hrsg.), Entwicklungen im Schweizer Blockchain-Recht (2021), S. 51, 82; Zellweger-Gutknecht/Monnerat, Internationaler Kontext: schweizerisches Registerwertrecht, $\$ 1$, in diesem Band.

17 Kuhn, in: Weber/ders. (Hrsg.), Entwicklungen im Schweizer Blockchain-Recht (2021), S. 51, 82. 
der Zustimmung der Gesellschaft technisch gar nicht erst möglich ist. ${ }^{18}$ Die einzige aktienrechtliche Änderung, die der Schweizer Gesetzgeber für erforderlich hielt, besteht im Erfordernis der statutarischen Verbriefungsklausel gem. Art. 622 Abs. 1 OR. Diese Klausel dient schlicht als gesellschaftsrechtliche Ausprägung der Registrierungsvereinbarung, so dass sich auf ihrer Grundlage eine Übertragung der Aktien durch Eintrag in einem Wertrechteregister vornehmen lässt. ${ }^{19}$ Sollen Aktien tokenisiert werden, fordert das Schrifttum zusätzlich eine Tokenisierungsklausel, die jene Registerwertrechte mit den digitalen Token verknüpft. ${ }^{20}$ Im Übrigen unterliegen tokenisierte Aktien jedoch vollumfänglich den herkömmlichen Regeln des Gesellschafts- bzw. Aktienrechts; auch kapitalmarktrechtliche Publizitätsvorschriften finden unverändert Anwendung. ${ }^{21}$ Gleichwohl gilt die Ausgestaltung von Aktien als Registerwertrechte als effizienzfördernd und kostensparend, weshalb diese Art der Aktienausgabe bzw. -verwaltung insbesondere von ersten FinTech- bzw. Start-up-Unternehmen bereits in Anspruch genommen worden ist. ${ }^{22}$

\section{b) Liechtenstein}

Auch Liechtenstein hat eine breitflächige, sogar noch übergreifendere KryptoGesetzgebung erlassen, nämlich das sog. Gesetz über Token und VT-Dienstleister (TVTG), das gem. Art. 51 bereits am 1.1.2020 in Kraft getreten ist. ${ }^{23}$ „VT“ steht gem. Art. 2 Abs. 1 lit.a) TVTG für vertrauenswürdige Technologien, die sich dadurch auszeichnen, dass sie „die Integrität von Token, die eindeutige Zuordnung von Token zu VT-Identifikatoren sowie die Verfügung über Token“ sicherstellen; unter VT-Systemen versteht das Gesetz „Transaktionssysteme, welche die sichere Übertragung und Aufbewahrung von Token

18 Botschaft (Fn.12), S. 274; Morscher/Staub, Tokenisierung von Effekten für Private Equity und Private Debt, in: Gericke/Europa Institut der Universität Zürich (Hrsg.), Venture Capital Reinvented - Markt, Recht, Steuern (2021), S. 193, https://doi.org/10.36862/eiz-357, S. 212.

19 Kubn, in: Weber/ders. (Hrsg.), Entwicklungen im Schweizer Blockchain-Recht (2021), S. 51,83 .

${ }^{20}$ Von der Crone/Monsch/Meisser, Aktien-Token, Eine privatrechtliche Analyse der Möglichkeit des Gebrauchs von DLT-Systemen, GesKR 2019, 1, 6.

21 So etwa Weber, RDi 2021, 186, 189.

22 Näher Weber/Kubn, in: dies. (Hrsg.), Entwicklungen im Schweizer Blockchain-Recht (2021), S. 285, 285. Vorerst überwiegt freilich noch die Zurückhaltung, weil der Teil der DLTGesetzgebung zum Aktienhandel erst am 1.8.2021 in Kraft tritt, vgl. Grundlebner, Die digitale Aktie ist da - und es läuft nicht wie erwartet, Neue Zürcher Zeitung v. 16. 3.2021, abrufbar unter https://www.nzz.ch/finanzen/aktien/die-digitale-aktie-ist-da-und-wenigpassiert-ld.1605958.

23 Zum TVTG näher Damjanovic/Pfurtscheller/Raschauer, Liechtensteins „BlockchainRegulierung“ - Ein- und Ausblicke, ZEuP 2021, 397; Deuber/Khorrami Jabromi, Liechtensteiner Blockchain-Gesetzgebung: Vorbild für Deutschland? Lösungsansatz für eine zivilrechtliche Behandlung von Token, MMR 2020, 576; Teichmann/Falker, Liechtenstein - Das TVTG und Risiken der Blockchain-Technologie, InTer 2020, 62. 
sowie darauf aufbauende Dienstleistungerbringung mittels vertrauenswürdiger Technologien ermöglichen“ (Art.2 Abs.1 lit.b) TVTG). Auf Grundlage dieser Terminologie statuiert Art. 2 Abs. 1 lit.c) TVTG eine Legaldefinition von Token als „Information auf einem VT-System, die: 1. Forderungs- oder Mitgliedschaftsrechte gegenüber einer Person, Rechte an Sachen oder andere absolute oder relative Rechte repräsentieren kann; und 2. einem oder mehreren VT-Identifikatoren zugeordnet wird“. ${ }^{24}$ Mit dieser Definition erschließt sich der überaus breite Anwendungsbereich des TVTG, weil sich nämlich jegliches übertragbare Recht als Token abbilden lässt, also nicht nur Rechte, die in Wertpapieren verbrieft werden können. Entsprechend lassen sich Mitgliedschaftsrechte aller Art tokenisieren, neben Aktien auch $\mathrm{GmbH}$-Anteile oder Anteile an anderen juristischen Personen oder Personengesellschaften. Zu diesem Zweck hat der liechtensteinische Gesetzgeber eine explizite Regelung zu Wertrechten in die gesellschaftsrechtliche Kodifikation eingefügt, \81a SchlT Personen- und Gesellschaftsrecht (PGR). ${ }^{25}$ Ähnlich der Rechtslage in der Schweiz kannte das liechtensteinische Recht freilich bereits zuvor unverbriefte Wertrechte, nachdem der Staatsgerichtshof bereits 1975 in einem obiter dictum alle Rechte, die nicht in Wertpapieren verbrieft sind, solchermaßen eingeordnet hatte. ${ }^{26}$ Umgekehrt ist die Verbriefung von Mitgliedschaftsrechten gem. Art. 150 Abs. 1 PGR nur mit gesetzlicher Erlaubnis zulässig; für Anteile an Aktiengesellschaften statuiert Art. 267 Abs. 1 PGR eine Pflicht zur Ausstellung von Aktienurkunden, die jedoch in den Statuten abbedungen werden kann. ${ }^{27} \mathrm{Im}$ Übrigen sah auch der liechtensteinische Reformgesetzgeber keinerlei Bedürfnis für gesellschaftsbzw. aktienrechtliche Anpassungen als Voraussetzung für die Zulassung tokenisierter Gesellschaftsanteile. Die ersten tokenisierten Aktien auf neuer gesetzlicher Grundlage wurden am 21. September 2020 generiert. ${ }^{28}$ Liechtenstein genießt gegenüber der Schweiz jedoch nicht nur zeitlichen Vorsprung, sondern bietet zudem den Vorteil der Mitgliedschaft im Europäischen Wirtschaftsraum (EWR): Infolge dieser EWR-Mitgliedschaft können entsprechend tokenisierte Gesellschaftsanteile, die von der liechtensteinischen Finanzaufsicht zum Handel zugelassen sind, aufgrund des Passporting-Regimes der EU-Prospektrichtlinie europaweit auf Kapitalmärkten gehandelt werden. ${ }^{29}$

${ }^{24}$ Vgl. dazu Damjanovic/Pfurtscheller/Raschauer, ZEuP 2021, 397, 407 f.; Deuber/Khorrami Jabromi, MMR 2020, 576, 578.

${ }^{25}$ Damjanovic/Pfurtscheller/Raschaner, ZEuP 2021, 397, $411 \mathrm{f}$.

26 StGH 1975/002 vom 29.04.1975, ELG 1973, 381, 384; dazu ausführlich Bergt, Token als Wertrechte (2. Aufl. 2020), S. 41.

27 Vgl. nochmals Bergt, Token als Wertrechte (2. Aufl. 2020), S. $34 \mathrm{f}$.

28 S. die Pressemitteilung v. 29.9.2020, „First equity tokens generated according to the Liechtenstein Token Act by Amazing Blocks", abrufbar unter https://my.amazingblocks.io/ blog/first-equity-tokens-according-to-the-liechtenstein-token-act-tokenized-by-amazingblocks.

${ }^{29}$ Näher Bergt, Token als Wertrechte (2. Aufl. 2020), S. $271 \mathrm{f}$. 


\section{Einsatzpotenzial}

Tokenisierte Aktien sind demnach zumindest in einzelnen Nachbarrechtsordnungen bereits Realität. Ob sie sich gegenüber herkömmlichen Gesellschaftsanteilen tatsächlich als effizienzfördernd und kostensparend erweisen, hängt von ihrem praktischen Einsatzpotenzial ab. Auch wenn Vorteile der (vor allem blockchain-basierten) Tokenisierung für verschiedene Anwendungsbereiche intensiv diskutiert werden, ${ }^{30}$ stehen insbesondere wertpapier- und kapitalmarktrechtliche Implikationen im Vordergrund, ${ }^{31}$ während das unternehmensinterne Anwendungspotenzial vorerst vergleichsweise wenig Beachtung findet. ${ }^{32}$ Sieht man von der Etablierung eigenständiger organisationaler Infrastrukturen wie den sog. Decentralized Autonomous Organisations (DAOs) ab, die keine Ergänzung, sondern eine Alternative zu herkömmlichen, rechtlich verfassten Gesellschaftsformen bilden, ${ }^{33}$ und beschränkt man sich auf den Einsatz entsprechender Technologien innerhalb solcher Gesellschaften durch Tokenisierung ihrer Gesellschaftsanteile, so lassen sich vor allem zwei Anwendungsbereiche unterscheiden, nämlich einerseits die Identifikation von Gesellschaftern und andererseits die Durchsetzung von gesellschaftsrechtlichen Pflichten. ${ }^{34}$

${ }^{30}$ Für eine praxisbezogene Einführung vgl. Tapscott/Tapscott, The Blockchain Revolution (2016).

${ }^{31} \mathrm{Zu}$ diesen Fragen etwa: Casper, Elektronische Schuldverschreibung: es ist Zeit für einen grundlegenden gesetzlichen Neustart, BKR 2019, 209; Möslein, Smart Contracts im Wertpapierhandelsrecht, in: FS 25 Jahre WpHG (2019), S. 465; Patz, Handelsplattformen für Kryptowährungen und Kryptoassets, BKR 2019, 435; Spindler, Initial Coin Offerings und Prospektpflicht und -haftung, WM 2018, 2109; Veil, Token-Emissionen im europäischen Kapitalmarktrecht, ZHR 183 (2019), 346.

32 S. jedoch Beurskens, Blockchain und Gesellschaftsrecht, in: FS Seibert (2019), S.71; ders., Blockchain und Gesellschaftsrecht - Endlose Möglichkeiten?, NZG 2021, 353; Guntermann, Die elektronische Aktie und das eWpG, AG 2021, 449, 452-454; Möslein, A Nexus of Smart Contracts? Gesellschaftsrechtspraxis und -theorie im Spiegel der Blockchain, in: FS Windbichler (2020), S. 889; ders./Omlor/Urbach, ZIP 2020, 2149; vgl. ferner auch Spindler, Gesellschaftsrecht und Digitalisierung, ZGR 2018, 17, 44-52; Zetzsche, Corporate Technologies - Zur Digitalisierung im Aktienrecht, AG 2019, 1, 3 f., $13 \mathrm{f}$.

${ }_{33}$ Dazu näher: Mann, Die Decentralized Autonomous Organization - ein neuer Gesellschaftstyp?, NZG 2017, 103; Möslein, A Nexus of Smart Contracts? Gesellschaftsrechtspraxis und -theorie im Spiegel der Blockchain, in: FS Windbichler (2020), S. 887, 895-898; Spindler, Blockchaintypen und ihre gesellschaftsrechtliche Einordnung, RDi 2021, 309; Teichmann, Digitalisierung und Gesellschaftsrecht, ZfPW 2019, 247, 266f.; demnächst monographisch: Mienert, Blockchain-basierte dezentrale autonome Organisationen und Gesellschaftsrecht, Diss. Marburg 2021.

${ }^{34}$ Hierzu sowie zum Folgenden bereits: Möslein, A Nexus of Smart Contracts? Gesellschaftsrechtspraxis und -theorie im Spiegel der Blockchain, in: FS Windbichler (2020), S. 887, 892-895. 


\section{a) Gesellschafteridentifikation}

Aufgrund ihrer Vorzüge als dezentrale, transparente Datenbank erleichtern Blockchain- und allgemein DLT-basierte Systeme die Identifikation von Gesellschaftern. Deren eindeutige Ermittlung ist Voraussetzung der zweifelsfreien Wahrnehmung von Gesellschafterrechten, etwa Stimm-, Informations- oder Klagerechten, aber auch umgekehrt der Durchsetzung gesellschaftsrechtlicher Ansprüche. Die herkömmliche Verwahrpraxis erschwert jedoch die Identifikation, weil Anteile typischerweise nicht direkt, sondern über eine Kette von Intermediären (den sog. Verwahrungsbaum) gehalten werden. ${ }^{35}$ Die zweite EU-Aktionärsrechte-Richtlinie (im Folgenden: 2. ARRL) operiert daher mit kaskadenartigen Informationspflichten. ${ }^{36}$ Entsprechend heißt es in $\$ 67 \mathrm{~d}$ Abs. 1 AktG, der die Richtlinienvorgabe in deutsches Recht umsetzt, börsennotierte Gesellschaft könnten „von einem Intermediär, der Aktien der Gesellschaft verwahrt, Informationen über die Identität der Aktionäre und über den nächsten Intermediär verlangen“. Je verästelter und vielgliedriger die Verwahrungsbäume sind, desto komplexer, aufwändiger und fehleranfälliger gerät die intermediärsbasierte Identifikation. ${ }^{37}$

Tokenisierte Anteile haben das Potenzial, diesen Aufwand zu reduzieren: Sind Gesellschafter in einer dezentralen Datenbank verzeichnet und schlagen sich dort auch Aktionärswechsel zuverlässig nieder, so lässt sich das Erfordernis „to know your shareholder" ungleich leichter erfüllen. ${ }^{38}$ Gesellschafter sind mittels der tokenisierten Anteile unmittelbar verfolgbar („traceable shares“). ${ }^{39}$ Es gibt längst Online-Plattformen, die solche Lösungen zu Zwecken von Stimm-

${ }^{35}$ Dazu ausführlich Zetzsche, Aktionärsidentifikation, Aktionärslegitimation und das Hauptversammlungsverfahren nach ARUG II, AG 2020, 1, 3.

${ }^{36}$ RL (EU) des Europäischen Parlaments und des Rates vom 17.5.2017 zur Änderung der RL 2007/36/EG im Hinblick auf die Förderung der langfristigen Mitwirkung der Aktionäre, ABl. EU Nr. L 132 v. 20.5.2017, 1; zur Bedeutung der Identifikation vgl. dort besonders Erwägungsgrund 4, S.2 („Voraussetzung für die unmittelbare Kommunikation zwischen den Aktionären und der Gesellschaft und deshalb von wesentlicher Bedeutung dafür, dass die Ausübung von Aktionärsrechten und die Mitwirkung der Aktionäre erleichtert werden“).

37 Der Anpassungsaufwand der IT-Systeme der Emittenten und Intermediäre erwies sich als so groß, dass der Gesetzgeber (trotz Ablaufs der Umsetzungsfrist) den Anwendungsbeginn der einschlägigen Vorschriften gem. Art. 26j Abs. 4 EGAktG eigens hinausgeschoben hat; vgl. dazu Zetzsche, AG 2020, 1.

${ }^{38} \mathrm{Zu}$ diesem Erfordernis etwa Bork, Die Regelungen zu „know-your-shareholder“ im Regierungsentwurf des ARUG II, NZG 2019, 738; Noack, Identifikation der Aktionäre, neue Rolle der Intermediäre - zur Umsetzung der Aktionärsrechte-Richtlinie II, NZG 2017, 561, $561 \mathrm{f} . ;$ Zetzsche, Know Your Shareholder, der intermediärsgestützte Aktionärsbegriff und das Hauptversammlungsverfahren - zur Umsetzung des Kapitels Ia der reformierten AktionärsrechteRL, ZGR 2019, 1.

39 Geis, Traceable Shares and Corporate Law, Nw. U. L. Rev. 113 (2018), 227 („poised to allow for specific share identification and precise records of share provenance"); ähnlich $D e$ Filippi/Wright, Blockchain and the Law (2018), S. 134. 
rechtsausübung und -vertretung anbieten. ${ }^{40}$ Aus Gründen der Rechtssicherheit ist wichtig, dass trotz der dezentralen Registerführung nur ausgewählte, vom Leitungsorgan ermächtigte Teilnehmer Transaktionen validieren können und das dezentrale Netzwerk deshalb in Form einer permissioned blockchain betrieben wird. Auch Geheimhaltungserfordernissen ist Rechnung zu tragen, etwa indem die Transparenz eingeschränkt wird und Nutzer nur per öffentlichem Schlüssel auftreten, so dass ohnehin Pseudonymität herrscht. ${ }^{41}$ Technische Gestaltungen, die jenen Anforderungen entsprechen, sind jedenfalls möglich und mindern keineswegs die Effizienzvorteile, die blockchain- gegenüber intermediärsbasierter Aktionäridentifizierung verspricht. ${ }^{42}$ Auch wenn sich dieser technische Schritt auf die Funktion der Datenaufzeichnung beschränkt, hat er weitreichende gesellschaftsrechtliche Bedeutung: „It will change the structure of shareholder lawsuits, alter the allocation of corporate governance rights, and require lawmakers to rethink fundamental principles of shareholder responsibility for corporate misdeeds“. ${ }^{43}$

\section{b) Durchsetzung von Gesellschafterrechten und-pflichten}

Über die Gesellschafteridentifikation hinaus lässt sich die Tokenisierung von Gesellschaftsanteilen nutzbar machen, um Gesellschafterrechte und -pflichten automatisiert durchzusetzen. Gesellschaftsrechtliche Regeln setzen präventive Verhaltensanreize, bedürfen im Konfliktfall aber auch effektiver Lösungs- und Durchsetzungsmechanismen. Blockchain- und DLT-basierte Tokenisierung eröffnet die Möglichkeit, jene Regeln in technischem Code abzubilden und dadurch ihre Durchsetzung zu automatisieren. ${ }^{44}$ Mit Hilfe von Smart Contracts lassen sich bei Eintritt bestimmter Parameter (etwa: Ausschüttungsbeschluss) Zins- bzw. Dividendenzahlungen automatisiert auslösen, was deren Verwal-

40 So bietet die elektronische Handelsplattform Nasdaq eine entsprechende e-VotingAnwendung an (allerdings vorerst nur in Südafrika, innerhalb der EU vorerst nur Pilotversuche in Estland), vgl. https://www.nasdaq.com/solutions/evoting-technology. Innerhalb der Citigroup wurde zu Zwecken der Stimmrechtsvertretung die Plattform Proximity entwickelt, s. https://www.citivelocity.com/proxymity/. Weitere Beispiele bei van der Elst/Lafarre, Blockchain and smart contracting for the shareholder community, EBOR 20 (2019), $111,130 \mathrm{f}$.

${ }_{41}$ Allgemein zur Pseudonymität: De Filippi/Wright, Blockchain and the Law (2018), S. $38 \mathrm{f}$.

42 Van der Elst/Lafarre, EBOR 20 (2019), 111, 133; s. außerdem Zetzsche, AG 2020, 1, 12 („Idealzustand“); Panisi/Buckley/Arner, Blockchain and Public Companies: A Revolution in Share Ownership Transparency, Proxy-Voting and Corporate Governance?, Stanford Journal of Blockchain Law \& Policy 2 (2019), 189.

${ }^{43}$ Geis, Nw. U. L. Rev. 113 (2018), 227.

${ }^{44}$ In diesem Sinne De Filippi/Wright, Blockchain and the Law (2018), S.133: „With a blockchain, organizations could decide to use code to implement parts of the organization's rules and procedures“. 
tung und Überwachung erheblich effizienter macht. ${ }^{45}$ Die Anwendungsmöglichkeiten beschränken sich nicht auf Vermögensrechte, sondern erstrecken sich auf die gesamte gesellschaftsrechtliche Organisationsverfassung. ${ }^{46}$ Insbesondere lassen sich gesellschaftsinterne Entscheidungsvorgänge automatisieren. ${ }^{47}$ So können Unternehmen die Software etwa von Boardroom nutzen, um die Entscheidungsfindung im Leitungsorgan $\mathrm{zu}$ organisieren. ${ }^{48}$ Soweit virtuelle Gesellschafterversammlungen zulässig sind, erstrecken sich die Anwendungsmöglichkeiten auch auf deren Beschlüsse. ${ }^{49}$ Tokenisierte Gesellschaftsanteile erleichtern die Einholung von Zustimmungsbeschlüssen, etwa bei eigeninteressierten Geschäften, und die Überwachung organisationsverfassungsrechtlicher Regeln. Anwendungen wie Otonomos eröffnen, soweit es die betreffende Gesellschaftsrechtsordnung zulässt, schon heute entsprechende technische Möglichkeiten: „Using Otonomos, people can form a corporation [...] that is entirely administered through a blockchain, including procedures related to voting, dividends, and capital increases“ ${ }^{50}$

\section{Reformperspektiven}

Dieses Potenzial tokenisierter, also blockchain- und DLT-basierter Gesellschaftsanteile hat im Grundsatz auch der deutsche Gesetzgeber erkannt. Bereits in der Blockchain-Strategie vom September 2019 stellte die Bundesregierung eine Prüfung der „Anwendung der Blockchain-Technologie im Gesellschaftsrecht" in Aussicht. ${ }^{51}$ Auf dieser Grundlage hat das Bundesministerium der Justiz und für Verbraucherschutz (BMJV) ein externes Gutachten eingeholt, das bis Ende 2020 die Anwendungsmöglichkeiten der Blockchain-Technologie im Gesellschafts- und Genossenschaftsrecht ausloten und Regelungsvorschläge „insbesondere zu Blockchain-Aktien [und] Blockchain-Geschäftsanteilen“ unterbreiten sollte, aber vorerst noch nicht veröffentlicht worden ist. ${ }^{52} \mathrm{Im} \mathrm{Ge}$ -

45 S. etwa Hacker/Thomale, Crypto-Securities Regulation: ICOs, Token Sales and Cryptocurrencies under EU Financial Law, ECFR 2018, 645, 650.

46 Ausführlich De Filippi/Wright, Blockchain and the Law (2018), S.133-136; vgl. außerdem Guntermann, AG 2021, 449, 453.

47 Dazu auch Beurskens, Blockchain und Gesellschaftsrecht, in: FS Seibert (2019), S.71, 89f.; Spindler, ZGR 2018, 17, 50.

48 Vgl. www.boardroom.to; dazu De Filippi/Wright, Blockchain and the Law (2018), S. 135; vgl. außerdem Enriques/Zetzsche, Corporate Technologies and the Tech Nirvana Fallacy, Hastings Law Journal 72 (2020), 55, $64 \mathrm{f}$.

49 Beurskens, Blockchain und Gesellschaftsrecht, in: FS Seibert (2019), S. 71, 90.

50 S. unter https://otonomos.com/. Zur Wahl stehen Gesellschaftsformen u. a. der Schweiz (GmbH), des Vereinigten Königreichs (LLP) sowie von Delaware (LLC und C-Corp.).

51 BT-Drs. 19/13433, S. 14.

52 Bei der Vergabe im Sommer 2020 wurden als Gutachter Prof. Dr. Philipp Maume, Prof. Dr. Sebastian Steinorst, Mathias Fromberger (alle Technische Universität München), Dr. Michael Denga (Humboldt-Universität zu Berlin) sowie Dr. Markus Kaulartz (CMS München) beauftragt; mit dem Erscheinen ihres Gutachtens ist noch in $2021 \mathrm{zu}$ rechnen. 
leitwort der Ministerin, das diesem Band vorangestellt ist, heißt es ebenfalls, auch für Aktien sei „eine zwingende Verbriefung [...] nicht länger zeitgemäß“. ${ }^{33}$ Allerdings seien, bevor auch für diese Wertpapiere eine elektronische Form vorgesehen werden könne, „noch weitere, anspruchsvolle Fragen zu klären“. 54 Gleichsinnig stellt auch der Regierungsentwurf des eWpG die „Regulierung von elektronischen Aktien“ bereits in Aussicht, wenngleich erst zu einem späteren Zeitpunkt. ${ }^{55}$ Immerhin sei der Entwurf bereits so gefasst, „dass eine spätere Öffnung für elektronische Aktien rechtsetzungstechnisch problemlos erfolgen" könne. ${ }^{56}$ Diesem Ziel soll ausdrücklich auch die Evaluierung der Gesetzesregeln dienen, die im Gesetz zwar nicht verpflichtend verankert ist, laut diesem Entwurf aber erfolgen soll, „sobald hinreichende Erfahrungen vorliegen, spätestens aber nach 5 Jahren ". ${ }^{57}$ Wenngleich sich der Gesetzgeber vorerst nicht zur Einführung elektronischer Gesellschaftsanteile durchringen konnte, weil er - ganz im Gegensatz zu den Nachbarrechtsordnungen - erheblichen gesellschaftsrechtlichen Anpassungsbedarf gesehen hat, scheint zumindest mittelfristig Aussicht auf eine entsprechende Reform zu bestehen, die eine Nutzung des Anwendungspotenzials solcher Anteile erlauben würde. Ob sich diese Aussicht in der nächsten (oder spätestens übernächsten) Legislaturperiode tatsächlich realisieren wird, wird freilich von vielerlei Faktoren abhängen, nicht zuletzt auch vom Ausgang der Bundestagswahl.

\section{Stillstand}

\section{Selbstbeschränkung des eWpG}

Vorerst bleibt dieser Reformschritt jedenfalls aus, weil sich das eWpG in $\$ 1$ selbst beschränkt, indem es seinen Anwendungsbereich auf Schuldverschreibungen auf den Inhaber begrenzt. Das Potenzial, das elektronische und besonders tokenisierte Gesellschaftsanteile versprechen, bleibt daher zunächst ungenutzt.

\section{a) Elektronische Aktien}

Die Frage nach den Gründen dieser auch im Vergleich zu den Nachbarrechtsordnungen bemerkenswerten Selbstbeschränkung beantwortet der Gesetzgeber im Hinblick auf elektronische Aktien vor allem mit Verweis auf „erhebliche

\footnotetext{
${ }^{53}$ Lambrecht, Geleitwort, in diesem Band.

${ }^{54}$ S. nochmals Lambrecht, Geleitwort, in diesem Band.

55 Bundesregierung, Begründung zum Entwurf eines Gesetzes zur Einführung von elektronischen Wertpapieren (2020), S. 41 (zu \$1), im Folgenden: RegE, abrufbar unter: https://www.bmjv.de/SharedDocs/Gesetzgebungsverfahren/DE/Einfuehrung_elektr_Wert papiere.html.

56 S. nochmals RegE, S. 41 (zu $\$ 1$ ).

57 RegE, S. 40.
} 
gesellschaftsrechtliche Auswirkungen“ ${ }^{58}$ die deren Einführung zur Folge hätte. Laut Entwurfsbegründung betreffen diese Auswirkungen „die Gründung der Gesellschaft, die Ausgabe von Aktien, die Übertragung der Aktien auf den internationalen Kapitalmärkten, die Einberufung der Hauptversammlung, Kapitalmaßnahmen sowie den Informationsfluss von der Gesellschaft zum Aktionär“. ${ }^{59}$ Ergänzend weist der Regierungsentwurf hinsichtlich des letztgenannten Punktes noch auf die europäischen Vorgaben hin, die mit ARUG II gerade erst umgesetzt worden seien und sich in der Praxis noch etablieren müssten. ${ }^{60}$

\section{b) Elektronische Geschäftsanteile}

Neben dieser ausdrücklichen Selbstbeschränkung des eWpG erfolgt eine weitere Eingrenzung - scheinbar - stillschweigend: Während die Nichterfassung elektronischer Aktien zumindest in der Gesetzesbegründung Erwähnung findet, finden andere elektronische Anteile und insbesondere elektronische Geschäftsanteile an $\mathrm{GmbH}$ und Genossenschaft in Gesetz und Begründung mit keinem einzigen Wort Erwähnung. Ins Auge fällt dieses Schweigen des Gesetzgebers vor allem in Gegenüberstellung zur Blockchain-Strategie und dem vom BMJV in Auftrag gegebenen Gutachten: Beide nehmen nämlich jeweils breitflächiger die „Anwendung der Blockchain-Technologie im Gesellschaftsrecht“ bzw. Geschäftsanteile von GmbHs und Genossenschaften in Bezug. ${ }^{61}$

Rechtsvergleichend greift der deutsche Gesetzgeber mit dieser Einschränkung freilich nur im Vergleich zu Liechtenstein kürzer, während in der Schweiz Gesellschaftsanteile, die nicht zugleich Wertpapiere sind, ebenfalls nicht in den Anwendungsbereich der Reformgesetzgebung fallen. ${ }^{62}$ Ausweislich seines Titels statuiert auch das deutsche Gesetz nur Regelungen „über elektronische Wertpapiere“. Aus diesem Grund sind andere elektronische Anteile von vorneherein außerhalb seines Anwendungsbereichs. Im Gegensatz zu Aktien sind $\mathrm{GmbH}$ - und genossenschaftsrechtliche Geschäftsanteile nämlich keine Wertpapiere. Sie kennen keinen wertpapier- und damit sachenrechtlichen Verkehrs-

${ }^{58}$ RegE, S. 41 (zu \$1); ebenso bereits Bundesministerium der Justiz und für Verbraucherschutz/Bundesministerium für Finanzen, Referentenentwurf eines Gesetzes zur Einführung von elektronischen Wertpapieren (2020), S. 35 (zu \$1); im Folgenden: RefE, abrufbar unter https://www.bmjv.de/SharedDocs/Gesetzgebungsverfahren/Dokumente/ RefE_Einfuehrung_elektr_Wertpapiere.html;jsessionid=87F7BB5F245A2D2087866A71995 445B8.2_cid297? $\mathrm{nn}=6712350$.

${ }^{59}$ RegE, S. 41 (zu \1); RefE, S. 35 f. (zu $\left.\$ 1\right)$.

60 BGBl. I 20191, S. 2637; vgl. RegE, S. 41 (zu \1); RefE, S. 36 (zu \$1).

61 BT-Drs. 19/13433, S. 14. Das BMJV hatte seinen Gutachtenauftrag „zur Anwendung der Blockckain-Technologie im Gesellschafts- und Genossenschaftsrecht" in einem Schreiben vom 24.4.2020 dahingehend konkretisiert, dass sich zur GmbH ähnliche Fragen stellten wie zur Aktiengesellschaft, „insbesondere zu Blockchain-Geschäftsanteilen und der Gründung, die auch für die Genossenschaft vertieft werden sollen“.

${ }^{62}$ S. oben, II.1.a) und b). 
schutz, da ihnen jedenfalls die Verbriefung fehlt. ${ }^{63}$ Ein gutgläubiger Erwerb von $\mathrm{GmbH}-$ Anteilen ist lediglich gem. $₫ 16 \mathrm{Abs} .3 \mathrm{GmbHG}$ möglich, wobei jedoch die Gesellschafterliste - nicht der verbriefte Anteil - als Rechtsscheinträger dient. ${ }^{64}$ Geschäftsanteile einer Genossenschaft verkörpern ohnehin nicht die Beteiligung, sondern bezeichnen lediglich den „Beteiligungshöchstbetrag“; 65 eine Übertragung der Mitgliedschaft bzw. deren Zweiterwerb sind bei Genossenschaften nicht möglich. ${ }^{66}$ Für eine Begründung, weshalb jene anderen Anteile nicht vom eWpG erfasst sind, bestand für den Gesetzgeber daher schlicht kein Anlass. Ob diese implizite Beschränkung des Anwendungsbereichs rechtspolitisch sinnvoll erscheint, ist damit freilich keineswegs gesagt, sondern bedarf ebenso der Diskussion wie der explizite Ausschluss elektronischer Aktien.

\section{Rechtspolitische Kritik}

Dass der Gesetzgeber Aktien - und auch Geschäftsanteile von GmbHs und Genossenschaften - nicht in den Anwendungsbereich des eWpG einbezogen hat, stieß bereits im Gesetzgebungsverfahren auf erhebliche Kritik. ${ }^{67}$ Ökonomisch erscheint fragwürdig, dass das eWpG die elektronische Begebung von Fremdkapital erleichtert, während wirtschaftspolitisch umgekehrt die Stärkung der unternehmerischen Eigenkapitalbasis wünschenswert erscheint, weil sie stärker zur Stabilität der Wirtschaft beiträgt. ${ }^{68}$ Rechtspolitisch lassen sich zudem eine ganze Reihe weiterer Argumente ins Feld führen.

\section{a) Internationaler Kontext}

Zunächst stellt die Selbstbeschränkung des deutschen Gesetzgebers zumindest hinsichtlich elektronischer Aktien einen rechtsvergleichenden Sonderweg dar, der im internationalen Regelungswettbewerb einen erheblichen Nachteil bedeutet. Die Entmaterialisierung von Wertpapieren ist in anderen europäischen Rechtsordnungen, etwa in Frankreich, ohnehin längst weiter fortgeschritten als in Deutschland. ${ }^{69}$ Der daraus resultierende Nachteil fällt besonders schwer

63 Möslein/Omlor/Urbach, ZIP 2020, 2149, 2157.

${ }^{64}$ Kritisch zu dieser Möglichkeit, die der Gesetzgeber erst mit der MoMiG-Reform von 2008 geschaffen hat: Omlor, Verkehrsschutz im Kapitalgesellschaftsrecht (2010), S. $331 \mathrm{ff}$., $523 \mathrm{ff}$.

${ }^{65}$ Beuthien, GenG (16. Aufl. 2018), $\$ 7$ Rn. 2.

66 Übertragbar ist lediglich das Geschäftsguthaben, vgl. $\int \mathbb{S} 15$ Abs. 1 S.1, 76 Abs. 1 S.1 GenG; dazu näher Bertram, in: Saenger/Aderhold/Lenkaitis/Speckmann (Hrsg.), Handelsund Gesellschaftsrecht (2. Aufl. 2011), Rn. $983 \mathrm{f}$.

67 S. dazu Arora (Deutsche Kreditwirtschaft), Hansen (Bitkom), Gebra (Immutable Insight), Liebscher (SdK) und Omlor, in: Deutscher Bundestag - Finanzausschuss, ProtokollNr. 19/124, bes. S. 5-7 und 11-13.

${ }^{68}$ Lehmann, Wertpapierarten, $\$ 3$, in diesem Band.

${ }^{69}$ Vgl. nur Lehmann, Zeitenwende im Wertpapierrecht, Der Referentenentwurf für ein Gesetz über elektronische Wertpapiere (eWpG), BKR 2020, 431, 432, m. w. N. 
ins Gewicht, weil und soweit das Passporting-Regime der EU-Prospektrichtlinie ein Ausweichen deutscher Emittenten insbesondere nach Liechtenstein erlaubt und sich die durch $\$ 1 \mathrm{eWp}$ bedingten Begrenzungen somit unschwer umgehen lassen. ${ }^{70}$ Die Verwaltungspraxis der BaFin, Token im Gegensatz zur Rechtsprechung unter den Voraussetzungen der Standardisierung und Fungibilität als Wertpapier sui generis zu behandeln, ${ }^{71}$ und die dadurch verursachte Rechtsunsicherheit schafft für deutsche Emittenten ohnehin Anreize, Tokenisierungsprojekte in anderen Staaten des Europäischen Wirtschaftsraums durchzuführen, um dort gebilligte Wertpapierprospekte anschließend - nach bloßer Notifizierung der BaFin - auch in Deutschland zu nutzen. ${ }^{72}$ Aufgrund der europäischen Vorgabe hat die Behörde keinerlei Möglichkeit, das öffentliche Angebot solcher Token in Deutschland zu untersagen oder von den Emittenten die Erstellung eines weiteren Prospekts zu verlangen. Auch auf die Begründung, die betreffenden Anteile unterfielen nicht dem Anwendungsbereich des eWpG, lässt sich eine Untersagung somit nicht stützen.

\section{b) Gesellschaftsrechtliche Auswirkungen}

Materiell-rechtlich stützt der Gesetzgeber die Beschränkung des Anwendungsbereichs des eWpG vor allem auf die befürchteten gesellschaftsrechtlichen Auswirkungen, die eine Einbeziehung elektronischer Aktien zur Folge hätte; zugleich suggeriert er, deren spätere Einbeziehung könne rechtsetzungstechnisch problemlos erfolgen. Angesichts dieses Spannungsfelds fragt sich, welche gesellschaftsrechtlichen Friktionen eine Öffnung des eWpG für elektronische Aktien verursachen würde.

Als mögliches Konfliktfeld benennt der Gesetzgeber insbesondere die „europäischen Vorgaben [...], die mit ARUG II gerade erst umgesetzt worden seien und sich erst noch in der Praxis etablieren müssten “. ${ }^{73}$ Jene Neuregelungen, die sich vor allem in dem neuen Identifikationsregime des $\$ 67 \mathrm{~d}$ AktG wiederfinden, dienen der Umsetzung der Richtlinienvorgabe des Art.3a Abs. 1 S. 1

70 Für eine praktische Handreichung vgl. etwa den Eintrag des Anbieters Amazing Blocks, „Tokenization in Liechtenstein with EU passporting“ v. April 2021, abrufbar unter https:// my.amazingblocks.io/blog/tokenization-in-liechtenstein-with-eu-passporting.

71 Vgl. einerseits Bundesanstalt für Finanzdienstleistungsaufsicht (BaFin), Fachartikel „Tokenisierung“ v. 15.4.2019, abrufbar unter https://www.bafin.de/SharedDocs/Veroef fentlichungen/DE/Fachartikel/2019/fa_bj_1904_Tokenisierung.html; BaFin, Hinweisschreiben (WA) „Aufsichtsrechtliche Einordnung von sog. Initial Coin Offerings (ICOs) zugrunde liegenden Token bzw. Kryptowährungen als Finanzinstrumente im Bereich der Wertpapieraufsicht", abrufbar unter: www.bafin.de/SharedDocs/Downloads/DE/Merkblatt/WA/ dl_hinweisschreiben_einordnung_ICOs.pdf;jsessionid=D8A3A7DEF916686A246D100311 121A60.1_cid372?__blob=publicationFile\&v=2; andererseits LG Berlin, BKR 2021, 170, 172.

72 Näher Buck-Heeb, Aktuelle Entwicklungen des Prospektrechts und der Prospekthaftung, BKR 2021, 317, 322; Heppding/Schalast/Schiereck, Rechtliche und regulatorische Handhabung von STO im internationalen Kontext, RdF 2021, 84.

73 Nachw. oben, Fn. 61. 
der 2. ARRL, nach der die Mitgliedsstaaten sicherstellen müssen, „dass Gesellschaften das Recht haben, ihre Aktionäre zu identifizieren“" ${ }^{74}$ Um dieses Recht zu gewährleisten, sollen insbesondere Intermediäre der Gesellschaft verpflichtet werden, auf deren Antrag hin unverzüglich Informationen über die Identität von Aktionären zu übermitteln, notfalls über die gesamte Verwahrkette hinweg (Art. 3a Abs. 2 und 3 der 2. ARRL). Die Tokenisierung von Aktien, die Intermediäre und Verwahrketten entbehrlich macht, ${ }^{75}$ steht mit dieser Vorgabe nur scheinbar in einem Spannungsverhältnis. Das eigentliche Regelungsziel, die Aktionärsidentifikation nach dem Grundsatz „know your shareholder“ zu ermöglichen, lässt sich durch Tokenisierung nämlich sogar ungleich besser erreichen, weil Aktionäre mit solchen „traceable shares“ unmittelbar verfolgbar sind. ${ }^{76}$ Indem die Durchführungsverordnung ausdrücklich den Einsatz neuer Technologien fordert, ebnet sie geradezu den Weg zu tokenisierten Aktien. ${ }^{77}$ Rechtlich besteht insofern keinerlei Spannungsfeld zur Richtlinienvorgabe. Angesichts des hohen Anpassungsaufwands, den die Implementierung der Vorgaben des $\$ 67 \mathrm{~d}$ AktG verursacht, hätte zudem auch praktisch viel dafür gesprochen, nach dem Vorbild ausländischer Gesetzgeber möglichst frühzeitig DLT-basierte Alternativen zu eröffnen, auch um Pfadabhängigkeiten zu vermeiden. ${ }^{78}$

Die „erhebliche[n] gesellschaftsrechtliche[n] Auswirkungen“, die der Gesetzgeber darüber hinaus mit Blick auf Gründung, Aktienausgabe und -übertragung, Einberufung der Hauptversammlung, Kapitalmaßnahmen sowie den Informationsfluss zum Aktionär ins Feld führt, ${ }^{79}$ erscheinen demgegenüber ungleich weniger substantiiert. ${ }^{80}$ Sie sind jedenfalls kein Hindernis für die Einfüh-

${ }^{74}$ Ausführlich insbesondere Bork, NZG 2019, 738; Foerster, Identifizierung der Aktionäre nach der Änderungsrichtlinie zur Aktionärsrechterichtlinie (2. ARRL) und dem Referentenentwurf ARUG II, AG 2019, 17; Noack, NZG 2017, 561.

75 S. dazu oben, II.2.a).

76 Vgl. nochmals oben, bei Fn. $39 f$.

77 In der Durchführungsverordnung (EU) 2018/1212 der Kommission v. 3.9.2018 zur Festlegung von Mindestanforderungen zur Umsetzung der Bestimmungen der Richtlinie 2007/36/EG des Europäischen Parlaments und des Rates in Bezug auf die Identifizierung der Aktionäre, die Informationsübermittlung und die Erleichterung der Ausübung der Aktionärsrechte (ABl. EU Nr. L 223 vom 4.9.2018, S. 1) heißt es im 4. Erwägungsgrund: „Um die Ausübung der Aktionärsrechte zu erleichtern und insbesondere grenzüberschreitend effizienter zu gestalten, sollte der Einsatz moderner Technologien für die Kommunikation zwischen Emittenten und ihren Aktionären und durch Intermediäre, einschließlich für diese Verfahren eingesetzter Dienstleistungserbringer, gefördert werden"; dazu auch van der Elst/ Lafarre, EBOR 20 (2019), 111, 113.

$78 \mathrm{Zu}$ entsprechenden Initiativen vor allem in Frankreich und den USA vgl. nochmals van der Elst/Lafarre, EBOR 20 (2019), 111, $131 \mathrm{f}$. Zweifelsohne wirft auch diese alternative Form der Aktionärsidentifikation rechtliche Anpassungs- und technische Gestaltungsfragen auf; s. dazu Möslein, A Nexus of Smart Contracts? Gesellschaftsrechtspraxis und -theorie im Spiegel der Blockchain, in: FS Windbichler (2020), S. 887, 895.

${ }^{79}$ Nachw. oben, Fn. 59.

${ }^{80}$ S. auch Möslein, in: Schwintowski, Bankrecht (6. Aufl. 2021), Kapitel 12, Rn. 60; ferner Guntermann, AG 2021, $455 \mathrm{f}$. 
rung elektronischer Gesellschaftsanteile, solange sich keine unlösbaren Regelungskonflikte dartun lassen. Die rechtsvergleichende Umschau liefert keinerlei Anhaltspunkte für solche Regelungskonflikte, haben sich die Gesetzgeber sowohl in der Schweiz als auch in Liechtenstein doch jeweils mit äußerst punktuellen gesellschaftsrechtlichen Modifikationen begnügen können, die sich auf das Erfordernis statutarischer Verbriefungsklauseln bzw. die deklaratorische Benennung von Wertrechten beschränken. ${ }^{81}$ Für das deutsche Aktienrecht ist schon deshalb wenig Anpassungsbedarf erkennbar, weil die Verbriefung von Aktien (anders als von Inhaberschuldverschreibungen) nicht rechtsbegründend wirkt. ${ }^{82}$ Weil zudem die Globalurkunde gem. \$9a DepotG den Regelfall bildet, heißt es im Schrifttum treffend: „Warum die in einem Tresor lagernde Globalurkunde die Gründung einer AG oder die Einberufung der HV erleichtern soll, ist kaum zu verstehen" ${ }^{83}$ Erste Vorarbeiten für ein Blockchain-Kapitalgesellschaftsrecht, die bereits vorliegen, ${ }^{84}$ belegen überdies, dass elektronische Aktien in der Tat „auch ohne eine große Reform des AktG [hätten] mitgenommen werden können “. ${ }^{85}$

Umgekehrt liegt keineswegs auf der Hand, dass die in Aussicht gestellte spätere Öffnung für elektronische Aktien rechtssetzungstechnisch problemlos möglich ist, schon weil Aktien gem. $\$ 10$ Abs. 1 AktG grundsätzlich als Namens-, nicht als Inhaberaktien begeben werden, das eWpG hingegen ausschließlich auf Inhaberpapiere zugeschnitten ist. ${ }^{86}$ Zudem drohen im Verhältnis von Kryptowertpapier- und Aktienregister Friktionen, ${ }^{87}$ die sich leichter bei Erlass als im Rahmen einer späteren Öffnung des eWpG hätten auflösen lassen. Die entsprechende Selbstbeschränkung des Gesetzgebers droht daher weitaus größere Pfadabhängigkeiten zu verursachen als die Gesetzesbegründung suggeriert.

\section{Verfassungsrechtliche Bedenken}

Die Eingrenzung des Anwendungsbereichs, die $\$ 1$ eWpG vorsieht, stößt nicht nur auf rechtspolitische Kritik, sondern weckt auch verfassungsrechtliche Bedenken. Die Beschränkung auf Inhaberschuldverschreibungen bedeutet näm-

81 Dazu ausführlich oben, II.1.

82 S. bereits RGZ 34, 110, 115 („nicht durch Vorhandensein von Aktienurkunden bedingt“); vgl. auch $\$ 214$ Abs. 4 S. 1 AktG („Gesellschaften, die keine Aktienurkunden ausgegeben haben“); vgl. dazu statt aller Reger/Langheld/Haagen, Elektronische Aktien, RDi 2021, 83, 87 .

83 Lehmann, BKR 2020, 431, 432; zustimmend bspw. Spindler, in: Möslein/Omlor (Hrsg.), FinTech-Handbuch (2. Aufl. 2021), \25 Rn. 43.

84 Möslein/Omlor/Urbach, ZIP 2020, 2149.

85 Casper, in: Möslein/Omlor (Hrsg.), FinTech-Handbuch (2. Aufl. 2021), \28, Rn. 76.

86 In diese Richtung auch Reger/Langheld/Haagen, RDi 2021, 83, $88 \mathrm{f}$.

87 Hierzu näher Möslein/Omlor/Urbach, ZIP 2020, 2149, 2155; Reger/Langheld/Haagen, RDi 2021, 83, 89. 
lich eine Ungleichbehandlung der verschiedenen unternehmerischen Finanzierungsmöglichkeiten, weil nur der Fremd-, nicht aber die Eigenkapitalaufnahme die Kostenvorteile der elektronischen Begebungsmöglichkeit zuteilwerden. Vor dem Hintergrund der unternehmerischen Finanzierungsneutralität, die das BVerfG erst kürzlich in einem Beschluss zur Einkünftekorrektur gem. $\$ 1$ Abs. 1 AStG auf Art. 3 Abs. 1 GG gestützt hat, ${ }^{88}$ weckt eine solche Ungleichbehandlung Bedenken, wenn sie sich nicht durch nachvollziehbare, sachgemäße Gründe rechtfertigen lässt. Ob diese Rechtfertigung im Fall des eWpG gelingt, erscheint angesichts der rechtspolitischen Kritik zumindest fraglich.

Nach dem Grundsatz der unternehmerischen Finanzierungsfreiheit steht es Gesellschaftern überdies grundsätzlich frei, ob und in welchem Umfang sie die Gesellschaft mit Eigen- oder mit Fremdkapital ausstatten. ${ }^{89}$ Als Ausprägung der in Art. 2 Abs. 1 GG geschützten Privatautonomie sowie der in Art. 12 Abs. 1 GG verbürgten unternehmerischen Betätigungsfreiheit ist dieser Grundsatz verfassungsrechtlich verbürgt: Er findet vor allem in der steuerrechtlichen Rechtsprechung vielfach Anwendung, seitdem der BFH in einem Urteil zur Gesellschafter-Fremdfinanzierung 1992 unter Berufung auf die damaligen Regelungen zu kapitalersetzenden Gesellschafterdarlehen entschieden hatte, eine Kapitalgesellschaft müsse über das gezeichnete Kapital hinaus nicht mit einer bestimmten Eigenkapitalquote ausgestattet sein. ${ }^{90} \mathrm{Ganz}$ ähnlich urteilte der Große Senat in einem Beschluss aus dem Jahr 1997, der Steuerpflichtige sei auch in der Verwendung von Eigen- und Fremdkapital frei: Aufgrund der Finanzierungsfreiheit sei eine Kontentrennung nicht missbräuchlich, selbst wenn der Steuerpflichtige alle Betriebseinnahmen auf ein Konto leite, von dem er Privatausgaben bestreitet, während Betriebsausgaben von einem anderen, defizitären Konto beglichen werden. ${ }^{91}$ Die Möglichkeit der Begebung elektronischer Schuldverschreibungen schränkt die Finanzierungsfreiheit zwar formal nicht ein, sondern eröffnet lediglich neue Gestaltungsmöglichkeiten (nur) für die Fremdkapitalaufnahme. Ähnlich wie Beschränkungen des Betriebsausgabenabzugs macht es aber die alternative Finanzierungsform, hier die Eigenkapitalaufnahme, teurer. Die daraus resultierende Beschränkung der unternehmerischen Finanzierungsfreiheit bedarf wiederum verfassungsrechtlicher Rechtfertigung. An der Verhältnis-

${ }^{88}$ BVerfG, NJW-RR 2021, 617, 618 (jedoch obiter dictum); grundlegend zum Spannungsverhältnis zwischen Finanzierungsfreiheit und -neutralität Herzig, Spannungsverhältnis zwischen Finanzierungsfreiheit und fehlender Finanzierungsneutralität der Besteuerung, FR 1994, 589; vgl. außerdem Musil/Leibohm, Die Forderung nach Entscheidungsneutralität der Besteuerung als Rechtsproblem, FR 2008, 807, 808.

89 Dazu aus unternehmensrechtlicher Sicht bspw. Luttermann, Unternehmen, Kapital und Genußrechte (1998), S.173; Baumbach/Hueck/Haas, GmbHG (22. Aufl. 2019), Anh. nach $\$ 64$, Rn. 1 .

90 BFH, NJW 1992, 2309, 2311.

91 BFH, NJW 1998, 1174; vgl. auch BFH, NJW 1998, 3590; einschränkend jedoch, im Anschluss an eine nachfolgende Gesetzesänderung: BFH NJW 1999, 671. 
mäßigkeit der Regelung bestehen nicht zuletzt deshalb erhebliche Zweifel, weil nicht einmal der Gesetzgeber selbst den Ausschluss elektronischer Aktien für erforderlich hält, sondern eine entsprechende Öffnung sogar bereits in Aussicht stellt. ${ }^{92}$ Die Scheu des Gesetzgebers vor befürchtetem - tatsächlich jedoch allenfalls punktuellem - gesellschaftsrechtlichen Anpassungsbedarf dürfte ohnehin keinen legitimen Zweck darstellen, mit dem sich Einschränkungen der unternehmerischen Finanzierungsfreiheit plausibel rechtfertigen ließen.

\section{Rückschritt?}

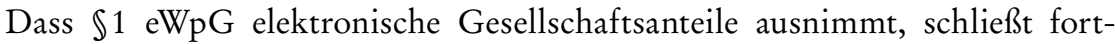
schrittliche Möglichkeiten der Unternehmensfinanzierung aus und bedeutet daher einen zumindest vorläufigen Stillstand. Möglicherweise erzwingt diese Beschränkung aber sogar einen Rückschritt gegenüber der bislang geltenden Rechtslage, weil das eWpG als argumentum e contrario bestehende, wenngleich umstrittene Spielräume künftig sogar einzuengen droht.

\section{Praxis elektronischer Gesellschaftsanteile, insbesondere: Equity Token}

In der Praxis wird der Effektengiroverkehr längst weitestgehend elektronisch abgewickelt. Anstelle der Übergabe von Urkunden finden reine Buchungsvorgänge statt. Gleichwohl gibt es noch Urkunden, die jedoch im Regelfall als Sammelurkunde bei einem Zentralverwahrer hinterlegt sind. Da Entmaterialisierung mithin nicht vollständig, aber doch schon sehr weitgehend erfolgt, spricht man treffend von einer „kunstvollen Übergangsform zum rein stückelosen Effektenverkehr“. ${ }^{3}$

Auch eine Tokenisierung von Gesellschaftsanteilen findet in der Praxis bereits statt. Als Sonderform der Security Token werden sog. Equity Token begeben, die Anteile an Gesellschaften abbilden. ${ }^{94}$ Nachdem das Berliner StartUp Neufund bereits im Jahr 2018 entsprechende Emissionen geplant, ${ }^{95}$ aber

92 S. dazu oben, unter III.2.b).

93 Hopt, in: Baumbach/Hopt (Hrsg.), HGB (35. Aufl. 2012), $\$ 1$ DepotG Rn. 2 (ebenso Kumpan jetzt in der 40. Aufl. 2021); ihm folgend Noack, in: Bayer/Habersack (Hrsg.), Aktienrecht im Wandel, Bd. 2 (2007), Kap. 11 Rn. 10; vgl. auch MüKo/Ekkenga, HGB, Bd. 6 (4. Aufl. 2019), Effektengeschäft, Rn. 38; ähnlich bereits Zöllner, Die Zurückdrängung des Verkörperungselements bei den Wertpapieren, in: FS Raiser (1974), S. 249, 255.

94 S. etwa Kaulartz, in: Möslein/Omlor (Hrsg.), FinTech-Handbuch (2. Aufl. 2021), \5 Rn.26; Nathmann, Token in der Unternehmensfinanzierung - Rechtliche Einordnung von Initial Coin Offerings (ICO), BKR 2019, 540, 548; allgemein auch Scherer, Blockchain im Wertpapierbereich (2020), S. 121-126.

${ }_{95} \mathrm{Vgl}$. hierzu das Whitepaper v. 2.0 von neufund.org, bes. S. 52, abrufbar unter https:// neufund.org/cms_resources/whitepaper.pdf; dazu auch Kaulartz/Matzke, Die Tokenisierung des Rechts, NJW 2018, 3278, 3280 (bes. Fn. 10). 
aufgrund regulatorischer Hürden schließlich aufgegeben hatte, ${ }^{96}$ wurde die ersichtlich erste von der BaFin genehmigte Equity-Token-Emission von der $p u-$ blity AG begeben. Ausweislich des öffentlichen Prospekts, der vom 23.11.2020 datiert und 520 Seiten umfasst, handelt es sich bei den sog. PREOS-Token um „auf den Inhaber lautende Blockchain basierte Wertpapiere sui generis [...], die (in Form eines tokenisierten Treuhandvermögens) durch Treuhand vermittelte wesentliche Aktionärsrechte sowie einen jederzeitigen Umtauschanspruch in Bezug auf bestehende, auf den Namen lautende Stammaktien ohne Nennbetrag (Stückaktien) der PREOS Global Office Real Estate \& Technology AG (Leipzig) (,PREOS-Aktien') aus dem Bestand der publity AG mit gleicher Gewinnanteilberechtigung wie alle anderen bestehenden Aktien der PREOS Global Office Real Estate \& Technology AG verkörpern“. ${ }^{97}$ Der Immobilieninvestor gilt als das weltweit erste börsennotierte Unternehmen, dessen Aktien durch einen blockchain-basierten Token digital verbrieft werden; zugleich sollte es sich um das größte Tokenisierungsprojekt in der Immobilienbranche weltweit handeln. ${ }^{98}$ Allerdings war die Emission nicht von Erfolg gekrönt: Laut Medienberichten wurden gerade einmal 1.613 Token im Gesamtwert von 14.100 Euro ausgegeben - während sich der Emittent 230 Millionen Euro erhofft hatte. ${ }^{99}$ Die BaFin untersuchte die Emission und hat am 31.3.2021 einen Warnhinweis veröffentlicht, in dem sie die Vermarktung des Tokens moniert: Es bestehe der Verdacht, dass seitens Dritter mit irreführenden, vom Prospekt abweichenden Aussagen für die Token geworben worden sei. ${ }^{100}$

Auch wenn blockchain-basierte Aktien demnach wirtschaftlich vorerst nicht unbedingt eine Erfolgsgeschichte gewesen sind, scheint das praktische Bedürfnis unverändert groß. Zuletzt hat die WEG Bank AG mit ihrer FinTech-Sparte TEN31 Bank eine Tokenisierung ihres Eigenkapitals angekündigt, mit der sie sogar noch einen Schritt weiter gehen möchte. Die Besonderheit ihrer sog. Blockchain Listed Shares (BLS) sei, so heißt es in einer Mitteilung vom 3.8.2021,

96 Näher: Klee, BTC Echo v. 23.6.2020, abrufbar unter https://www.btc-echo.de/news/ neufund-zu-tode-reguliert-94310/ („Neufund - zu Tode reguliert?“).

97 Der Prospekt ist zusammen mit zwei Nachträgen sowie u.a. mit den Treuhand- und Tokenbedingungen abrufbar unter https://www.publity.org/de/preos-token-oeffentlichesangebot-wertpapier-prospekt/. Vgl. dazu auch Guntermann, AG 2021, 449.

98 So jedenfalls Schmitz, BlockchainInsider v. 1.12.2020, abrufbar unter https://www. blockchain-insider.de/weltweit-erste-tokenisierung-von-aktien-eines-boersennotierten-un ternehmens-a-983200/.

99 Eich, DerTreasurer v. 14.4.2021, abrufbar unter https://www.dertreasurer.de/news/ finanzierung-corporate-finance/preos-und-publity-was-ist-los-bei-den-token-pionieren2018231/.

100 BaFin, Mitteilung v. 31.3.2021, abrufbar unter https://www.bafin.de/SharedDocs/ Veroeffentlichungen/DE/Verbrauchermitteilung/weitere/2021/meldung_210331_PREOS. html („PREOS-Token und Aktien der PREOS Global Office Real Estate \& Technology AG: Hinreichend begründeter Verdacht für Werbeverstöße"). Darüber hinaus hat die Behörde Untersuchungen wegen mutmaßlicher Marktmanipulation eingeleitet. 
„dass der Eintrag auf der Blockchain der tatsächliche Eigentumsnachweis in Bezug auf die jeweilige Aktie ist“. ${ }^{101}$ BLS, so heißt es weiter, sei die „,erste Blockchain-basierte Technologie, die einem Anleger einen direkten Zugriff auf die gehaltenen Aktien“ gebe; dies ermögliche „ein weit höheres Maß an Rechtssicherheit als dies bei herkömmlichen Verfahren der Tokenisierung möglich gewesen wäre“. ${ }^{102}$ Der Wertpapierprospekt ist von der BaFin gebilligt worden; die Emission der 2,83 Mio. jungen Aktien soll im Oktober 2021 erfolgen.

\section{Zulässigkeit im geltenden Recht}

Tokenisierte Gesellschaftsanteile sind mithin bereits gelebte Praxis, während das eWpG elektronische Aktien erst in Aussicht stellt. Aus diesem Spannungsfeld resultiert die Frage, ob bzw. in welcher Gestaltung solche Anteile im geltenden Recht zulässig sind. Umgekehrt formuliert lautet die Überlegung, welche rechtlichen Zulässigkeitsgrenzen eine künftige Öffnung des eWpG überhaupt noch zu überwinden verspricht. Ähnlich wie die Praxis des Effektengiroverkehrs dürften die derzeitigen Equity Token vorerst allenfalls eine „kunstvolle Übergangsform“103 zu echten Krypto-Anteilen sein; möglicherweise sind sie auch nur ein „fauler Kompromiss“. ${ }^{104}$

\section{a) Virtuelle Gesellschaftsanteile}

Equity Token vermitteln typischerweise keine echte gesellschaftsrechtliche Beteiligung und unmittelbare Gesellschafterstellung. Ähnlich den sog. phantom stocks (Phantomaktien), die seit langem der Mitarbeiterbeteiligung dienen, ${ }^{105}$ handelt es sich stattdessen um rein virtuelle Gesellschaftsanteile, die die Beteiligungsrechte von Gesellschaftern nur durch schuldrechtliche Vereinbarung nachbilden. PREOS-Token liefern ein anschauliches Beispiel: Sie vermitteln die fraglichen Aktionärsrechte nachweislich des Prospekts „durch Treuhand“ und verkörpern zudem einen Umtauschanspruch in Bezug auf PREOS-Aktien. ${ }^{106}$ Zwischen dem Emittenten und dem Token-Inhaber besteht mithin eine lediglich schuldrechtliche Beziehung, die als Treuhandverhältnis, alternativ aber bei-

101 Abrufbar unter https://www.dgap.de/dgap/News/corporate/weg-bank-durchbruchbei-der-tokenisierung-von-eigenkapital-weg-bank-begibt-neue-aktien-als-blockchainlisted-shares-bafin-genehmigt-wertpapierprospekt/?newsID=1468124.

102 Vgl. nochmals Nachw. Vorn.

103 Nachw. oben, Fn. 94.

104 Staub/Canaris, Großkomm. z. HGB (2. Aufl. 1981), Bankvertragsrecht, Rn. 2040; ähnlich Zöllner, Die Zurückdrängung des Verkörperungselements bei den Wertpapieren, in: FS Raiser 1974, S. 249, 255 („,im Grunde nur ein Denkbehelf, eine geistige Krücke“).

105 Näher dazu Feddersen, Aktienoptionsprogramme für Führungskräfte aus kapitalmarktrechtlicher und steuerlicher Sicht, ZHR 161 (1997), 269, 285; Hoppe, Zustimmungspflichten der Hauptversammlung bei der Ausgabe von Phantom Stocks und anderen virtuellen Beteiligungsformen, NZG 2018, 811, $811 \mathrm{f}$.

106 Nachw. oben, Fn. 98. 
spielsweise auch als Unterbeteiligung bzw. stille Gesellschaft ausgestaltet sein kann. ${ }^{107}$

\section{b) Unmittelbare Beteiligung, insbesondere durch Blockchain Listed Shares}

Als entscheidendes Hindernis für die Einräumung einer unmittelbaren Beteiligung gelten gemeinhin die bestehenden Formvorschriften - bei Aktien $\ 185$ Abs. 1 S. 1 AktG, der Schriftform des Zeichnungsscheins erfordert, bei GmbHAnteilen $\$ 15$ Abs. 3 GmbHG, der für die Abtretung von Geschäftsanteilen die notarielle Form vorsieht. ${ }^{108}$ Weil solche Formerfordernisse bei Personengesellschaften fehlen (bzw. die Eintragung in das Handelsregister lediglich deklaratorischer Natur ist), soll sich bei diesen hingegen „auch über die Blockchain eine Gesellschafterstellung vermitteln“ lassen. ${ }^{109}$ In Wahrheit kommt Formerfordernissen für die Tokenisierung im (Kapital-)Gesellschaftsrecht jedoch keineswegs die Schlüsselrolle zu. Sie erschweren zwar die Übertragung der betreffenden Anteile, schließen deren Tokenisierung aber nicht a priori aus. Sinn dieser Erschwernis sind bestimmte Formzwecke, die unabhängig von der technischen und rechtskonstruktiven Ausgestaltung gelten: So soll die notarielle Form den Transfer von GmbH-Anteilen erschweren, damit diese nicht Gegenstand spekulativen Handels werden; zudem soll die Meldung des beurkundenden Notars Fiskalinteressen des Staates absichern und dessen neutrale Beratung Verbraucher- und Anlegerschutz gewährleisten. ${ }^{110}$ Um diese Zwecke auch bei Tokenisierung zu wahren, empfiehlt sich statt der Streichung der Formvorschriften deren kreative Befolgung, etwa indem der Zugang zur GmbH-Blockchain über den Notar erfolgt. ${ }^{111}$

Hinderlicher für die Tokenisierung wären demgegenüber zwingende Verbriefungserfordernisse. Weil (im Gegensatz zu sonstigen Gesellschaftsanteilen) lediglich Aktien Wertpapiercharakter zukommt, ${ }^{112}$ stellt sich die Frage solcher Erfordernisse ohnehin nur für das Aktienrecht. Auch wenn das Aktiengesetz teils davon spricht, dass Aktien „ausgestellt werden“ (vgl. \$23 Abs. 3 Nr. 5 AktG; ähnlich $\$ 199$ AktG: „Ausgabe von Bezugsaktien), wirkt die Verbriefung von Aktien, anders als die Verbriefung von Inhaberschuldverschreibungen, nicht

107 Vgl. Guntermann, AG 2021, 449; Spindler, in: Möslein/Omlor (Hrsg.), FinTechHandbuch (2. Aufl. 2021), \$25, Rn.43; Werneburg, Steuerliche Behandlung von Erträgen aus Security Token, BB 2019, 2844, 2846; Wolf, Initial Coin Offerings (2020), S. 54f., 100-108.

108 S. nochmals Spindler, in: Möslein/Omlor (Hrsg.), FinTech-Handbuch (2. Aufl. 2021), \$25 Rn. 43; Werneburg, BB 2019, 2844, 2846.

109 Spindler, in: Möslein/Omlor (Hrsg.), FinTech-Handbuch (2. Aufl. 2021), \$25 Rn.43; Teichmann, ZfPW 2019, 247, 249 f.; einschränkend für Kommanditanteile hingegen Werneburg, BB 2019, 2844, 2846 (aufgrund des Handelsregisterzwangs nur mittelbar).

110 Näher: Möslein/Omlor/Urbach, ZIP 2020, 2149, 2158.

111 S. nochmals Möslein/Omlor/Urbach, ZIP 2020, 2149, 2158: Notare als zentrale Anlaufstelle („one-stop-shop“).

112 Vgl. oben, III.1. 
rechtsbegründend. ${ }^{113}$ Die Verbriefung ist lediglich von deklaratorischer Bedeutung. Entsprechend erkennt $\$ 214$ Abs. 4 S. 1 AktG ausdrücklich die Existenz von Aktiengesellschaften an, „die keine Aktienurkunden ausgegeben haben“. Wie das Reichsgericht bereits im Jahr 1894 festgestellt hat, ist die Entstehung von Aktien deshalb „nicht durch das Vorhandensein von Aktienurkunden bedingt“. ${ }^{114}$ Eine andere Frage ist jedoch, ob die Aktionäre einen Anspruch auf Verbriefung geltend machen können. Ein Anspruch auf Verbriefung des einzelnen Anteils kann in der Satzung ausgeschlossen oder eingeschränkt werden, wie \10 Abs. 5 AktG (und ähnlich $\$ 10$ Abs. 1 Nr. 2 AktG für Inhaberaktien) ausdrücklich statuiert. ${ }^{115}$ Umstritten ist jedoch, ob ein Anspruch auf Verbriefung in einer Sammelurkunde besteht, also Verbriefung sämtlicher Anteile derselben Art und Gattung in einer einzigen Globalurkunde verlangt werden kann. Bei nicht-börsennotierten Inhaberaktien sind solche Sammelurkunden (und deren Hinterlegung bei einer der benannten Stellen) gem. $\$ 10$ Abs. 1 S. 2 Nr. 2 AktG seit 2016 schon von Gesetzes wegen erforderlich. ${ }^{116}$ Die herrschende Meinung bejaht jedoch auch ansonsten einen - satzungsfesten! - Anspruch des Aktionärs auf Verbriefung sämtlicher Anteile in einer Globalurkunde. ${ }^{117}$ Mit der Reform des $₫ 10$ Abs. 5 AktG habe der Gesetzgeber nämlich nicht zum Wertrecht übergehen wollen, sondern habe die Praxis der Sammelverwahrung als Grundlage des Effektengiroverkehrs im Blick gehabt. ${ }^{118}$ Da Wertpapiere kapitalmarktrechtlich keiner Verbriefung bedürfen, streitet in der Sache vor allem das Aktionärsinteresse an der Möglichkeit des gutgläubigen Erwerbs für die Bejahung eines solchen Anspruchs: Fehlt jegliche Urkunde, lassen sich Aktien dinglich nämlich nur durch schlichte Einigung nach $\$ \int 413,398$ BGB ohne Gutglaubensschutz übertragen. ${ }^{119}$ Eine Mindermeinung hält diese Begründung für nicht

113 BeckOGK/Vatter (Stand: 1.6.2021), \$10 AktG Rn. 32f.; Hüffer/Koch, AktG (15. Aufl.

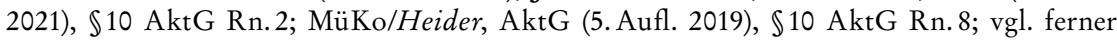
Nachw. Fn. 83.

114 RGZ 34, 110, 115.

115 Näher etwa BeckOGK/Vatter (Stand: 1.6.2021), \10 AktG Rn. 87; MüKo/Heider, AktG (5. Aufl. 2019), $\$ 10$ AktG Rn. 6.

116 Ihrig/Wandt, Die Aktienrechtsnovelle 2016, BB 2016, 6, 7; BeckOGK/Vatter (Stand: 1.6.2021), \10 AktG Rn.11.

117 Vgl. statt aller: OLG München NZG 2005, 765; BeckOGK/Vatter, AktG (Stand: 1.6.2021), $\$ 10$ AktG Rn. 88; Hüffer/Koch, AktG (15. Aufl. 2021), $\$ 10$ AktG Rn. 3, 12f.; MüKo/ Heider, AktG (5. Aufl. 2019), $\$ 10$ AktG Rn.60; Seibert, Der Ausschluß des Verbriefungsanspruchs des Aktionärs in Gesetzgebung und Praxis, DB 1999, 267, 269; Maul, Zur Ausgabe von Namensaktien, NZG 2001, 585.

118 IdS BT-Drs. 13/10038, S. 25: „Damit wird nicht der Schritt zum Wertrecht unter Abkehr vom Wertpapier vollzogen, denn der Ausschluß der Verbriefung betrifft lediglich den jeweiligen Anteil der einzelnen Aktionäre“; vgl. außerdem BeckOGK/Vatter, AktG (Stand: 1.6.2021), $₫ 10 \mathrm{AktG}$ Rn. 88 .

119 BeckOGK/Vatter, AktG (Stand: 1.6.2021), \10 AktG Rn.88; vgl. außerdem Möslein/ Omlor/Urbach, ZIP 2020, 2149, 2159; Guntermann, AG 2021, 449, 450 f. 
mehr zeitgemäß und verneint insbesondere für nicht-börsennotierte Aktiengesellschaften ein Bedürfnis nach entsprechendem Verkehrsschutz. ${ }^{120}$

Selbst wenn man den Anspruch auf Verbriefung der Anteile in einer Sammelurkunde bejaht, stellt sich die Folgefrage, ob sich dieser Anspruch nicht bereits nach geltendem Recht durch die Erfassung der Aktien in einem Register, das vergleichbaren Gutglaubensschutz gewährleistet, erfüllen lässt. ${ }^{121}$ In diesem Fall wäre denkbar, die schriftliche Urkunde durch Eintrag auf der Blockchain zu ersetzen. In Anlehnung an die Regelungen zur Gesellschafterliste der $\mathrm{GmbH}$ kommt möglicherweise das Aktienregister gem. $\$ 67 \mathrm{AktG}$ als ein solcher Rechtsscheinträger in Betracht. Nach den einschlägigen Formanforderungen des $\$ 239$ HGB darf es gem. Abs. 4 auch auf Datenträgern geführt werden, sofern die Daten während der Dauer der Aufbewahrungsfrist verfügbar sind und jederzeit innerhalb angemessener Frist lesbar gemacht werden können. ${ }^{122}$ Diese Voraussetzungen erfüllt die Blockchain ohne Weiteres. ${ }^{123}$ Auf das Aktienregister bauen dann auch die Blockchain Listed Shares der WEG-Bank auf, von denen es heißt, „dass der Eintrag [...] der tatsächliche Eigentumsnachweis in Bezug auf die jeweilige Aktie ist". ${ }^{124}$ Indessen unterscheidet sich das Aktienregister als Mitgliederverzeichnis der Aktiengesellschaft in seiner Rechtswirkung grundlegend von der Gesellschafterliste als vergleichbarem Verzeichnis der GmbH, das unter Mitwirkung eines Notars beim Handelsregister einzureichen ist und gem. $\$ 16$ Abs. 3 GmbHG als Rechtsscheinträger dient. ${ }^{125}$ Dem Aktienregister, das durch die Gesellschaft selbst oder durch einen beauftragten Dienstleister, also nicht hoheitlich, sondern privat geführt wird, kommt gem. $\$ 67 \mathrm{Abs} .2 \mathrm{~S} .1 \mathrm{AktG}$ zwar gesellschaftsinterne Legitimationsfunktion zu; es entfaltet aber mangels Gutglaubenstatbestand keinerlei Auswirkung auf die materielle Rechtslage. ${ }^{126}$ Um eine digitale Verbriefung mittels blockchain-basierter Aktien zu ermöglichen, müsste zumindest die relative Wirksamkeit der Aktienübertragung in Fortführung dieser Vorschrift und in Anlehnung an $\$ 8$ Abs. 1 BSchuWG von der Eintragung in einem blockchain-basierten Aktienregister abhängig gemacht werden. ${ }^{127} \mathrm{Ob}$ eine solche Fortentwicklung im Wege der Rechtsfortbildung möglich ist, erscheint mehr als zweifelhaft. Eine Analogie zu $\$ 952 \mathrm{AktG}$ dürfte

120 So insbesondere Noack, Globalurkunde und unverkörperte Mitgliedschaften bei der kleinen Aktiengesellschaft, in: FS Wiedemann (2002), S. 1141, 1148-1151; Schwennicke, Der Ausschluß der Verbriefung der Aktien bei der kleinen Aktiengesellschaft, AG 2001, 118, 124.

121 In diese Richtung Reger/Langheld/Haagen, RDi 2021, 83, 87.

122 MüKo/Bayer, AktG (5. Aufl. 2021), $\$ 67$ AktG Rn. 14; Hüffer/Koch, AktG (15. Aufl. 2021), 110 AktG Rn.4; Leuering, Das Aktienbuch, ZIP 1999, 1745; vgl. außerdem BT-Drs. 14/4051, S. 10 („elektronisch geführte Datenbanken“).

123 Beurskens, NZG 2021, 353; vgl. auch Reger/Langheld/Haagen, RDi 2021, 83, 89.

124 Nachw. oben, Fn. 102.

125 Möslein/Omlor/Urbach, ZIP 2020, 2149, 2159.

126 Rachlitz/Grigoleit, in: Grigoleit (Hrsg.), AktG (2. Aufl. 2020), \$67 Rn. 64.

127 Ausführlicher Möslein/Omlor/Urbach, ZIP 2020, 2149, 2160. 
das methodengerechte Maß überspannen, so dass es mutmaßlich einer gesetzgeberischen Spezialregelung bedarf. ${ }^{128}$

\section{3. $e W p G$ e contrario?}

Wenngleich elektronische Gesellschaftsanteile in der Praxis bereits existieren und sogar die BaFin bereits entsprechende Wertpapierprospekte gebilligt hat, ${ }^{129}$ ist jedenfalls die tokenbasierte Einräumung einer unmittelbaren Gesellschaftsbeteiligung rechtlich mit erheblichen Unsicherheiten behaftet. Diese Unsicherheiten betreffen einerseits den von der Mehrheitsansicht bejahten Anspruch auf Ausstellung einer Sammelurkunde. Weil eine solche Urkunde der Verwahrung bedarf, reduziert ein entsprechendes Erfordernis die Effizienzvorteile tokenisierter Aktien erheblich. Andererseits bestehen große Zweifel, ob die Blockchain eine solche Sammelurkunde zu ersetzen vermag, indem sie dem Aktienregister zumindest gewisse Auswirkung auf die materielle Rechtslage verleiht. Beide Rechtsfragen werden überwiegend zu Ungunsten tokenisierter Aktien beantwortet, sind aber umstritten und vorerst nicht höchstrichterlich entschieden. ${ }^{130}$ Die Genehmigung entsprechender Prospekte durch die BaFin schafft keine gegenläufigen Indizien, weil sich der Prüfungsumfang der Behörde gem. $\$ 20$ Abs. 4 ProspektVO darauf beschränkt, ob alle prospektrechtlich vorgeschriebenen Angaben im Prospekt enthalten (Vollständigkeitsprüfung) und verständlich dargelegt sind (Verständlichkeitsprüfung); zudem wird noch geprüft, dass sich die im Prospekt enthaltenen Angaben inhaltlich nicht widersprechen (Kohärenzprüfung). ${ }^{131}$ Eine Prüfung der zivilrechtlichen Implikationen und insbesondere der Möglichkeiten gutgläubigen Erwerbs erfolgt hingegen nicht.

Ist die zivilrechtliche Zulässigkeit tokenisierter Aktien bislang ungeklärt, so vermag der Erlass des eWpG jedenfalls nicht unmittelbar zur Klärung beitragen, weil sich der Anwendungsbereich nicht auf solche Anteile erstreckt. Der in der Gesetzesbegründung manifestierte Wille des Gesetzgebers, die Regulierung elektronischer Aktien zu einem späteren Zeitpunkt in Angriff zu nehmen, liefert jedoch zusätzliche Argumente gegen eine solche Zulässigkeit. Diese Selbstbeschränkung belegt nämlich, dass die langjährige Forderung nach Aktien-Wertrechten vom Gesetzgeber einmal mehr zurückgewiesen wird. ${ }^{132}$

128 So jedenfalls Möslein/Omlor/Urbach, ZIP 2020, 2149, 2160.

129 S. oben bei Fn. $102 \mathrm{f}$.

130 Vgl. nochmals Nachw. Fn. 117-121 bzw. Fn. 127-129.

131 Dazu statt aller: Buck-Heeb, Kapitalmarktrecht (11. Aufl. 2020), Rn. 225; Poelzig, Kapitalmarktrecht (2. Aufl. 2021), Rn. 251.

132 In diese Richtung bereits Zöllner, Die Zurückdrängung des Verkörperungselements bei den Wertpapieren, in: FS Raiser (1974), S.249; Staub/Canaris, Großkomm. z. HGB (2. Aufl. 1981), Bankvertragsrecht, Rn. 2042; Westermann, Das Girosammeldepot im deutschen Recht, RabelsZ 49 (1985), 226. Exemplarisch für die gesetzgeberische Zurückhaltung: 
Durch Rechtsfortbildung zu einem Ergebnis zu kommen, zu dem sich der Gesetzgeber selbst ausdrücklich (noch) nicht durchringen konnte, erscheint methodisch umso weniger haltbar. Obgleich elektronische Aktien und allgemein Gesellschaftsanteile außerhalb seines Anwendungsbereichs verbleiben, könnte das eWpG daher ein argumentum e contrario gegen deren Zulässigkeit liefern. ${ }^{133}$ Das Gesetz versäumt auf diesem Gebiet nicht nur, zum Fortschritt beizutragen, und verursacht stattdessen Stillstand, sondern dürfte - zumindest argumentativ - sogar einen Rückschritt bedeuten. Das zögerliche Stückwerk des Gesetzgebers erweist sich insofern als übervorsichtig. Mehr Mut wagen, so sollte daher spätestens in der nächsten Legislaturperiode das gesetzgeberische Motto in Sachen elektronischer Gesellschaftsanteile lauten.

\section{Zusammenfassung in Thesen}

1. Elektronische Gesellschaftsanteile zählen, selbst wenn sie sich als Wertpapiere qualifizieren, nicht zu den elektronischen Wertpapieren, die das eWpG regelt. Der Gesetzestitel verspricht mehr, als das Gesetz halten kann: Aufgrund der Beschränkung des $\$ 1 \mathrm{eWpG}$ handelt es sich lediglich um ein Gesetz über elektronische Inhaberschuldverschreibungen.

2. Rechtsvergleichend treten vor allem die Nachbarrechtsordnungen Schweiz und Liechtenstein mit einschlägigen Regelungen hervor. Tokenisierte Aktien sind in beiden Ländern bereits Realität: In der Schweiz gelten alle Rechte, die in Wertpapieren verbrieft werden können, als registerwertrechtefähig; nach dem liechtensteinischen TVTG lässt sich sogar jedes übertragbare Recht als Token abbilden. In beiden Rechtsordnungen waren gleichwohl nur sehr punktuelle gesellschaftsrechtliche Anpassungen erforderlich.

3. Die Tokenisierung von Gesellschaftsanteilen erweist sich als effizienzfördernd und kostensparend, weil sie aufgrund der Vermeidung von Verwahrketten die Identifikation von Gesellschaftern erleichtert und zugleich eine automatisierte Durchsetzung von Gesellschafterrechten und -pflichten erlaubt.

4. Dieses Potenzial hat nachweislich der Gesetzesbegründung - sowie bereits der Blockchain-Strategie - auch der deutsche Gesetzgeber erkannt. Gleichwohl konnte er sich vorerst nicht zur Einführung elektronischer Gesellschaftsanteile durchringen, weil er im Gegensatz zu den Nachbarrechtsordnungen

BT-Drs. 13/10038, S. 25; überblicksweise zu den Entwicklungslinien: Noack, in Bayer/Habersack (Hrsg.), Aktienrecht im Wandel, Bd. 2 (2007), Kap. 11 Rn. 13.

133 Methodisch verdient eine Lehre vom ,allgemeinen negativen Satz“, nach der jede Nichtregelung zugleich eine negative Regelung bedeutet, zwar keine Anerkennung; vgl. dazu nur Canaris, Die Feststellung von Lücken im Gesetz (2. Aufl. 1983), S. 37. Aufgrund der ausdrücklichen Gesetzesbegründung sind die methodischen Umstände jedoch zumindest im Falle elektronischer Aktien anders. 
erheblichen gesellschaftsrechtlichen Anpassungsbedarf gesehen hat. Während er dies mit Blick auf elektronische Aktien explizit begründet, sind andere elektronische Anteile, insbesondere elektronische Geschäftsanteile an $\mathrm{GmbH}$ und Genossenschaft, im Gegensatz zur liechtensteinischen Regelung bereits deshalb nicht vom Anwendungsbereich des eWpG erfasst, weil es sich bei diesen Anteilen nicht um Wertpapiere handelt.

5. Der rechtsvergleichende Sonderweg, den die fehlende Regelung elektronischer Aktien - und teils auch sonstiger Gesellschaftsanteile-bedeutet, wird sich im internationalen Regelungswettbewerb als nachteilig erweisen. Der Nachteil wiegt umso schwerer, als das europäische Passporting-Regime ein Ausweichen deutscher Emittenten insbesondere nach Liechtenstein erlaubt.

6. Die Eingrenzung des Anwendungsbereichs stößt vor allem deshalb auf rechtspolitische Kritik, weil die Berufung auf drohende gesellschaftsrechtliche Auswirkungen nicht schlüssig erscheint. Insbesondere besteht kein Spannungsfeld zu den Vorgaben der 2. ARRL, die der deutsche Gesetzgeber mit dem ARUG II umgesetzt hat. Vorarbeiten zu einem Blockchain-Kapitalgesellschaftsrecht zeigen zudem, dass elektronische Aktien unschwer ins eWpG hätten einbezogen werden können.

7. Auch verfassungsrechtlich weckt die Eingrenzung des Anwendungsbereichs Bedenken. Die Ungleichbehandlung unterschiedlicher unternehmerischer Finanzierungsformen ist insbesondere mit dem Grundsatz unternehmerischer Finanzierungsfreiheit schwer zu vereinbaren, der durch Art. 2 Abs. 1 GG und Art. 12 Abs. 1 GG verfassungsrechtlich verbürgt ist. Angesichts der rechtspolitischen Kritik dürfte eine Rechtfertigung auf Ebene der Verhältnismäßigkeit nicht gelingen.

8. Tokenisierte Aktien sind gleichwohl bereits gelebte unternehmerische Praxis. Als Equity Token vermitteln sie allerdings typischerweise keine unmittelbare Gesellschafterstellung. Vielmehr handelt es sich um virtuelle Gesellschaftsanteile, die Beteiligungsrechte von Gesellschaftern lediglich durch schuldrechtliche Vereinbarung nachbilden.

9. Entscheidendes Hindernis für die Einräumung unmittelbarer Gesellschaftsbeteiligungen sind weniger die vieldiskutierten Formerfordernisse, sondern zwingende Verbriefungserfordernisse. Im Aktienrecht ist umstritten, ob ein satzungsfester Anspruch der Aktionäre auf Verbriefung sämtlicher Anteile in einer Globalurkunde besteht. Weil eine Verbriefung die Möglichkeit gutgläubigen Aktienerwerbs eröffnet, bejaht die herrschende Meinung einen solchen Anspruch. Eine Ersetzung der Globalurkunde durch ein blockchain-basiertes Aktienregister, wie sie offenbar im Fall der sog. Blockchain Listed Shares geplant ist, ist nicht möglich, weil bzw. soweit das Aktienregister gem. $\$ 67$ Abs. 2 S. 1 AktG lediglich gesellschaftsinterne Legitimationswirkung entfaltet. 
10. Sowohl beim Streit um einen satzungsfesten Verbriefungsanspruch als auch bei der Frage einer rechtsfortbildenden Ausweitung der Rechtswirkungen des Aktienregisters liefert der in der Gesetzesbegründung manifestierte Wille des Gesetzgebers, elektronische Aktien vorerst nicht zu regeln, ein zusätzliches argumentume contrario gegen deren Zulässigkeit. Insofern versäumt das eWpG auf dem Gebiet elektronischer Gesellschaftsanteile nicht nur, zum Fortschritt beizutragen, indem es Stillstand verursacht. Zumindest argumentativ bedeutet das Zögern des Gesetzgebers sogar einen Schritt zurück. 


\title{
Elektronische Fondsanteile
}

\author{
Thomas Eckhold
}

I. Überblick . . . . . . . . . . . . . . . . . . . . . . . . . . . . . . . . . . . . . . 207

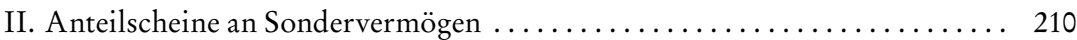

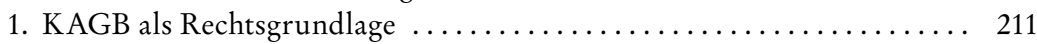

2. Registerbegebung mit Fiktionswirkung $\ldots \ldots \ldots \ldots \ldots \ldots \ldots \ldots \ldots 213$

3. Vorrang des KAGB vor dem eWpG .................... 216

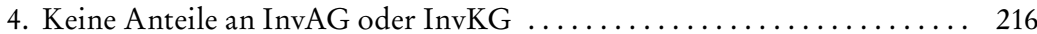

5. Inhaberfondsanteile ................................. 217

6. Sammel- nicht Einzeleintragung $\ldots \ldots \ldots \ldots \ldots \ldots \ldots \ldots \ldots \ldots \ldots 218$

7. Anteilsklassen und Teilsondervermögen $\ldots \ldots \ldots \ldots \ldots \ldots \ldots \ldots 219$

8. Zentralregisterfondsanteile . ........................ 220

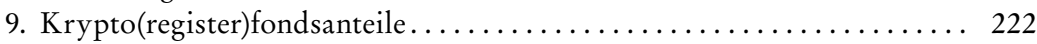

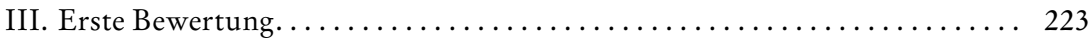

\section{I. Überblick}

Erstmals in ihrer Unterrichtung zur Blockchain-Strategie kündigte die Bundesregierung im September 2019 unter dem Oxymoron „elektronische Wertpapiere" eine auf Schuldverschreibungen begrenzten Öffnung des deutschen Rechts für registrierte, digital dargestellte und gespeicherte Werte an. ${ }^{1}$ Das Kompositum der beiden sich widersprechenden Begriffe „elektronisch“ einerseits und „Wertpapier" andererseits soll dabei schon sprachlich verdeutlichen, dass trotz der Ersetzung der Verbriefung durch registerhafte Digitalisierung einer Rechtsposition der sachenrechtliche Regelungsansatz des Wertpapierrechts beibehalten wird. ${ }^{2}$ Die Rechtswirkungen einer elektronischen Begebung und Speicherung sollen den Rechtswirkungen der Begebung durch papierhafte

${ }^{1}$ Bundesregierung, Blockchain-Strategie. Wir stellen die Weichen für die Token-Ökonomie, 19.9.2019, BT-Drs. 19/13433. Vgl. zu grds. Überlegungen zu Wertrechten Lehmann, Finanzinstrumente - von Wertpapier- und Sachenrecht zum Recht der unkörperlichen Gegenstände (2009); Segna, Bucheffekten (2013), S. $27 \mathrm{ff}$.

2 Vgl. dazu Einsele, Allgemeines Privatrecht, $\$ 2$ und Omlor, Verfügungen über elektronische Wertpapiere, $\$ 6$, beide in diesem Band; Labusen, Das Sachenrecht der elektronischen Wertpapiere, RDi 2021, $161 \mathrm{ff}$.; Meier, Übertragung von elektronischen Wertpapieren nach dem eWpG-E, RDi 2021, 1 ff. m. w. N. 
Verkörperung entsprechen. Offengehalten werden soll damit bewusst die Entscheidung, ob die über eine Rechtsfiktion ermöglichte „Brückenlösung“ der Versachlichung von digitalen Codes letztlich „in Richtung eines buchungsbasierten Rechts der Kapitalmarkt-Wertpapiere" weist ${ }^{3}$ - was allerdings zu wünschen wäre.

Es verwundert daher nicht, dass sich weder in der Blockchain-Strategie noch im diese vorbereitenden Eckpunktepapier des BMF/BMJV für die regulatorische Behandlung von elektronischen Wertpapieren vom März 2019 (Eckpunktepapier) ${ }^{4}$ Überlegungen zu dem hier zu erörternden speziellen Oxymoron „elektronischer Anteilscheine“ an Investmentvermögen fanden. Entsprechend beschränkte sich der Referentenentwurf eines Gesetzes zur Einführung von elektronischen Wertpapieren vom 10. August 2020 (RefE) ${ }^{5}$ in seinem Kernstück des Art. 1 auf den Entwurf eines Gesetzes über elektronische Wertpapiere für Schuldverschreibungen auf den Inhaber. Begleitänderungen waren in den Art. 2 bis 9 für das durch die Einführung elektronischer Wertpapiere betroffene Aufsichts- und Prospektrecht vorgesehen. Die Einführung „elektronischer Investmentfondsanteile" sollte ursprünglich erst in einem nächsten Schritt anstehen, ${ }^{6}$ wenn auch Regelungen für Aktien und andere Formen von Wertpapieren, die mitgliedschaftliche Rechte verkörpern, hinsichtlich ihrer Eignung zur Entmaterialisierung überprüft werden sollen. ${ }^{7}$

Dieser auf Schuldverschreibungen begrenzte und auf dem Sachenrecht aufsetzende Ansatz wurde und wird vielfach als insgesamt zu beschränkt, ${ }^{8}$ aber

3 Begr. RegE eWpG, BT-Drs.- 19/26925 (zitiert anhand der unlektorierten Vorabfassung), S. 43; kritische Auseinandersetzung hiermit bei Lehmann, Wertpapierarten, $\mathbb{3}$, in diesem Band; Darstellung des schweizerischen Regelungsansatzes bei Zellweger-Gutknecht/Monnerat, Internationaler Kontext: Schweizerisches Registerwertrecht, $\mathbb{1} 1$, in diesem Band.

${ }^{4}$ Abrufbar über die Internetseite des BMF unter: https://tinyurl.com/yzvn5xal (alle Hyperlinks in diesem Beitrag wurden zuletzt am 15.08.2021 abgerufen); dazu Casper, Elektronische Schuldverschreibung: es ist Zeit für einen grundlegenden gesetzlichen Neustart, BKR 2019, $209 \mathrm{ff}$.

${ }_{6}$ Abrufbar über die Internetseite des BMF unter: https://tinyurl.com/yg6bhxvr.

${ }^{6}$ So die Formulierung in Bundesregierung, Blockchain-Strategie (Fn. 1), S. 6.

7 Zur Entmaterialisierung des Wertpapiers und Re-statt Dematerialisierung des Sachenrechts siehe zuletzt Omlor, Re- statt Dematerialisierung des Sachenrechts, RDi 2021, $236 \mathrm{ff}$. m.w.N.

${ }^{8}$ Zum RefE eWpG teils lobend teils kritisierend etwa Lehmann, Zeitenwende im Wertpapierrecht, BKR 2020, 431 ff.; Saive, Einführung elektronischer Wertpapiere, ZRP 2020, $219 \mathrm{ff}$; Preuße/Wöckener/Gillenkirch, Der Gesetzesentwurf zur Einführung elektronischer Wertpapiere, BKR 2020, $551 \mathrm{ff} . ;$ Segna, Elektronische Wertpapiere im zentralen Register, WM 2020, 2301 ff.; Döding/Wentz, Der Referentenentwurf zur Einführung von elektronischen Wertpapieren und Kryptowertpapieren, WM 2020, 2312 ff.; Linardatos, Elektronische Schuldverschreibungen auf den Inhaber - des Wertpapiers neue Kleider, ZBB 2020, $329 \mathrm{ff}$.; Sickinger/Thelen, Anleihen und Genussscheine auf die Blockchain, AG 2020, $862 \mathrm{ff}$.; Voß, Der Regierungsentwurf des eWpG und das Depotrecht - Ein Warnruf, ZdiW 1 (2021), $16 \mathrm{ff}$; D ubovitskaya, Gesetzentwurf zur Einführung von elektronischen Wertpapieren: ein zaghafter Schritt nach vorn, ZIP 2020, 2551 ff.; Kleinert/Mayer, Der deutsche Weg zum elek- 
auch im Besonderen mit Blick auf das Recht der Investmentfonds kritisiert. ${ }^{9}$ Während aus investmentrechtlicher Perspektive noch Verständnis dafür bestand, die Einführung elektronischer Anteilscheine mitgliedschaftlich organisierter Investmentvermögen aufgrund ihres Zusammenhangs mit dem Gesellschaftsrecht bis zu dessen digitaler Aufarbeitung zurückzustellen, ${ }^{10}$ wurde Unverständnis darüber geäußert, auch Anteile an Sondervermögen aus dem Anwendungsbereich der elektronischen Wertpapiere auszuklammern. Letztere wiesen eine hinreichende Vergleichbarkeit mit Schuldverschreibungen auf, so dass eine Erstreckung des Anwendungsbereichs des eWpG auf Anteile an Sondervermögen auf den Inhaber nicht nur sinnvoll, sondern zukunftsgerichtet geradezu notwendig sei. ${ }^{11}$

Dieser Ansicht hat sich in der Folge die Bundesregierung in ihrem Gesetzesentwurf vom 16. Dezember 2020 eines Gesetzes zur Einführung von elektronischen Wertpapieren (RegE) zwar angeschlossen. ${ }^{12}$ Der RegE sah in einem gegenüber dem RefE neu aufgenommenen Art. 10 eine Änderung des KAGB vor, die erstmalig die Möglichkeit schaffen sollte, „elektronische Anteilscheine“ auf den Inhaber für Sondervermögen auszugeben. Allerdings sah der RegE entgegen verbreiteter Forderungen diese nur als Zentralregisterwertpapiere und nicht als Kryptowertpapiere vor. ${ }^{13}$ Hieran hielt trotz anhaltender Kritik die Beschlussempfehlung und der Bericht des Finanzausschusses vom 05. Mai 2021 fest, sah aber erweiternd jetzt die Möglichkeit vor, zukünftig durch Rechtsverordnung Inhaberanteilscheine an Sondervermögen auch als „Kryptoanteilschein“ einzuführen und hierdurch „Kryptofondsanteile“ zuzulassen. ${ }^{14}$ Auch behielt der RegE das grundsätzliche Konzept des wertpapier- und damit sa-

tronischen Wertpapier, EuZW 2020, 1059 ff.; zum RegE Labusen, RDi 2021, $161 \mathrm{ff}$; Meier, RDi 2021, $1 \mathrm{ff}$.

9 Vgl. bspw. Stellungnahme des BVI zum RefE-eWpG, GZ: VII B 5 - WK 6100/20/10001: 004, DOK: 2020/0779342, S. 1, abrufbar über die Internetseite des BMF unter: https://tinyurl. com/yj4gxfou; Stellungnahme des BAI zum RegE eWpG, GZ: VII B 5 - WK 6100/20/10001 :004 DOK: 2020/0779342, S. 2, abrufbar über die Internetseite des BMF unter: https://tinyurl. com/yh79tymn; Lehmann, BKR 2020, 431, 432.

10 Zur Kritik hieran siehe Möslein, Elektronische Gesellschaftsanteile, $\$ 8$, in diesem Band.

11 Stellungnahme des BVI (Fn. 9), S. 1; Stellungnahme des BAI (Fn. 9), S. 2.

12 Begr. RegE eWpG, BT-Drs. 19/26925 (zitiert anhand der unlektorierten Vorabfassung), Art. 10, S. 28, 86f.; vgl. auch Wieneke/Kunz, Das Gesetz zur Einführung von elektronischen Wertpapieren, NZG 2021, 316, 317; Mittwoch, Der Entwurf eines Gesetzes zur Einführung elektronischer Wertpapiere - ein Quantensprung für das Zivil- und Finanzmarktrecht?, WM $2021,375,378$.

${ }^{13}$ Begr. RegE eWpG (Fn. 12) Art. 10, $\$ 95$ Abs. 1 bis 4 KAGB n. F., S. 28, 86f. Zur Unterscheidung von Zentralregister- und Kryptowertapieren siehe Habersack, Zentrales Register vs. Kryptowertpapierregister, $\mathbb{\$} 4$, in diesem Band.

14 Beschlussempfehlung und Bericht des Finanzausschusses eWpG, BT-Drs. 19/29372 (zitiert anhand der unlektorierten Vorabfassung), Art. 10, $\$ 95$ Abs. 5 KAGB n.F., S. 45, 61; vgl. dazu die Presseerklärung des BAI vom 6. Mai 2021, abrufbar über die Internetseite des BAI unter: https://bit.ly/3hdPu7u. 
chenrechtlichen Ansatzes bei, wenngleich in der technischen Umsetzung zahlreiche Kritikpunkte des RefE beseitigt wurden. ${ }^{15}$

Das Gesetz zur Einführung von elektronischen Wertpapieren und damit das eWpG und die hier zu erörternden Änderungen des KAGB wurde schließlich am 6. Mai 2021 in der Fassung der Beschlussempfehlung des Finanzausschusses verabschiedet und am 09. Juni 2021 verkündet, so dass es am 10. Juni 2021 in Kraft getreten ist. ${ }^{16}$ Erwartet wird vielfach nun eine „Epoche der Tokenisierung“ verbunden mit dem Wunsch „eine Legislaturperiode der Tokenisierung beginnen zu lassen“. ${ }^{17}$

\section{Anteilscheine an Sondervermögen}

Wesentlicher, ja nahezu einziger eigenständiger Regelungsgegenstand des Gesetzes zur Einführung von elektronischen Wertpapieren ist aus Sicht des KAGB als Kerngesetz des Investmentgeschäfts eine Erweiterung von $\$ 95 \mathrm{KAGB}$, deren Umsetzung auf dem eWpG aufsetzt. Insoweit folgt der Gesetzgeber den Vorschlägen aus der Praxis, zumindest Anteile an Sondervermögen in den Öffnungsprozess des Wertpapierrechts einzubeziehen.

$\$ 95$ KAGB regelte schon in seinem bisherigen Absatz 1 Satz 1 sowohl die Pflicht zur als auch in den Sätzen 2 bis 6 die Art und Weise der (papierhaften) Verbriefung von Anteilen an einem Sondervermögen, als gesetzliche Grundlage für den Wertpapiercharakter eines ordnungsgemäß verbrieften Anteilscheins. ${ }^{18}$ Anteilscheine waren und bleiben auch zukünftig danach Anteile an Sondervermögen, die durch den bislang in $\$ 95$ Abs. 1 Sätze 2 bis 6 KAGB a. F. und jetzt in $\$ 95$ Abs. 2 KAGB geregelten Dokumentationsakt mit dem Papier verbunden und so zur Anwendung sachenrechtlicher Grundsätze verkörpert werden. ${ }^{19}$ Anteilscheine sind danach Bescheinigungen über den Anteil i.S. einer beweisenden (= sichtbar und verkörperte) Urkunde. Keine inhaltlichen Änderungen erfahren auch die in Absatz 2 a. F. geregelten rechtlichen Wirkungen der Über-

15 Überblick zu den Änderungen zwischen RefE und RegE bei Mittwoch, WM 2021, $375 \mathrm{ff}$. m. w. N.

16 Beschlussfassung ausgewiesen im Plenarprotokoll 19/227 v. 6. Mai 2021, S. 28952. Inkrafttreten geregelt in Art. 12 des Gesetzes zur Einführung von elektronischen Wertpapieren v. 6. Mai 2021, BGBl. I S. 1423; Überblick dazu bei Lehmann, Das Gesetz zur Einführung von elektronischen Wertpapieren, NJW 2021, $2318 \mathrm{ff}$.

17 Omlor, Elektronische Wertpapiere nach dem eWpG, RDi 2021, 371; wohl zurückhaltender Lebmann, NJW 2021, 2318, 2323 Rn. 51 und Lebmann, Wertpapierarten, $\$ 3$ I, in diesem Band.

18 Vgl. exempl. Emde/Dornseifer/Dreibus/Stabenow, KAGB (2. Aufl. 2019), \95 Rn.3; Baur/Tappen/Mehrkhah/Behme/v. Schweinitz/Schneider-Deters, InvestmentG, Bd.2 (4. Aufl. 2020), $\$ 95$ Rn. 11, 14.

${ }_{19}$ Zum bisherigen Recht Assmann/Wallach/Zetzsche/Zetzsche/Nast, KAGB (2019), $\$ 95$ Rn. 5 . 
tragung solcherart verkörperter Anteilscheine. ${ }^{20}$ Diese Regelungen finden sich, da das bisherige Wertpapier- und Depotrecht fort gilt, heute im Wesentlichen unverändert in $\$ 95$ Abs. 4 KAGB - auf die Verbriefung und ihre Rechtsfolgen ist hier daher nicht weiter einzugehen.

\section{KAGB als Rechtsgrundlage}

Um nun auch elektronische Anteilscheine zu ermöglichen, ordnet $\$ 95$ Abs. 1 Satz 1 KAGB neuerdings an, dass Anteile an Sondervermögen entweder in Anteilscheinen verbrieft oder als elektronische Anteilscheine begeben werden müssen. Hierdurch kann die weiterhin bestehende Pflicht zur Schaffung wertpapierrechtlicher Verkehrsfähigkeit aufgrund Anwendung sachenrechtlicher Grundsätze entweder durch Verkörperung (Papier) oder digitalisierte registerrechtliche Verdinglichung (Buchungscode) der Anteile an einem Sondervermögen wahlweise durch Verbriefung oder durch elektronische Begebung erfüllt werden. Die Kompetenz zur Begebung solcher elektronischer Anteilscheine leitet sich damit unmittelbar positiv aus dem KAGB und nicht aus dem eWpG ab.

Dies ist folgerichtig, denn es ist die Aufgabe des KAGB, den Regelungsrahmen des Investmentgeschäfts und damit auch die Rechtsgrundlage zur Erschaffung elektronischer Investmentfondsanteile zu setzen. ${ }^{21}$ \95 Abs. 1 Satz 1 KAGB begründet damit eine vom eWpG losgelöste eigenständige Rechtsgrundlage für elektronische Anteilscheine, obwohl die Kritiker des RefE eine Ausweitung des Anwendungsbereichs des eWpG gefordert hatten. ${ }^{22}$ Weder sind Anteilscheine mit Schuldverschreibungen gleichzusetzen, weshalb der Anwendungsbereich nach $₫ 1 \mathrm{eWp}$ ohne die ausdrückliche Anordnung des $\ 95 \mathrm{Abs} .1$ Satz 1 KAGB nicht eröffnet ist, noch bringt $\int 95$ Abs. 3 Satz 1 KAGB die Begebungskompetenz des $\$ 2$ Abs. 1 Satz $1 \mathrm{eWpG}$ zur Anwendung, ${ }^{23}$ sondern diese wird durch die eigenständige Rechtsgrundlage des $₫ 95$ Abs. 1 Satz 1 KAGB ersetzt. Dies unterstreicht die gesetzgeberische Absicht, dass das eWpG bewusst kein „Gesetz aus einem Guss für alle kapitalmarktgehandelten Finanzinstrumente“ darstellt, ${ }^{24}$ sondern ein funktionaler Ansatz verfolgt wird, bei dem et-

20 Vgl. exempl. Weitnauer/Boxberger/Anders/Anders, KAGB (3. Aufl. 2021), KAGB \95 Rn. 2; Assmann/Wallach/Zetzsche/Zetzsche/Nast, KAGB (2019), \95 Rn. 1.

21 Wie hier Lehmann, Wertpapierarten, $\$ 3$ II 2, in diesem Band. Hingegen kritisch zum gewählten Regelungsansatz und eine in das KAGB ausgelagerte Einbeziehung in das eWpG annehmend Omlor, RDi 2021, $371 \mathrm{f}$. („Kodifikationstechnisch ungeschickt“), allerdings ohne sich mit den damit verbundenen Rechtswirkungen auseinanderzusetzen.

22 Stellungnahme BVI (Fn. 9), S. 4; Stellungnahme BAI (Fn. 9), S. 4.

23 Dies belegt nun auch die noch eingefügte Rechtsverordnungskompetenz in $\$ 95$ Abs. 4 KAGB (dazu unter II.9.), wonach Kryptofondsanteile erst über eine gesonderte Rechtsverordnung möglich gemacht werden können, die sich dann aber ebenfalls auf das KAGB und nicht das eWpG stüzt.

24 So bspw. die rechtspolitisch nachvollziehbare Forderung von Lebmann, BKR 2020, 431. 
waigen spezifischen Regelungen des jeweiligen Wertpapiers Rechnung getragen werden soll. ${ }^{25}$

Hiermit soll erkennbar auch eine analoge Anwendung der elektronischen Begebungsform auf andere als im eWpG ausdrücklich genannte Wertpapierarten unterbunden werden. Regelungslücken zu Wertpapieren über Schuldverschreibungen und Anteile an Sondervermögen hinaus sind bewusst, so dass sich ein Analogieschluss selbst im Falle einer vergleichbaren Interessenlage verbietet. Dies belegt nun zudem $\$ 95$ Abs. 5 KAGB. Denn auch wenn eine verbreitete Meinung annimmt, dass Anteile an Sondervermögen einer Begebung als Kryptowertpapiere zugänglich sein sollten, behält es das KAGB dem Verordnungsgebern BMF und BMJV vor, die Vergleichbarkeit der Interessenlage zu prüfen und durch Erlass einer „maßgeschneiderten“ Rechtsverordnung etwaige Lücken zu schließen. ${ }^{26}$

Dieser Regelungsansatz trägt den Charakteristiken von Anteilen an Sondervermögen Rechnung. Lässt sich nämlich einerseits die Vergleichbarkeit von Anteilscheinen an Sondervermögen mit Schuldverschreibungen betonen, indem ihre Unterschiede zu Anteilscheinen hervorgehoben werden, die eine mitgliedschaftliche Beteiligung an einem Investmentvermögen verbriefen, ${ }^{27}$ unterscheiden sie sich andererseits gleichwohl von Schuldverschreibungen: Anteilscheine verbriefen nicht nur schuldrechtliche Ansprüche, sondern jedenfalls im Falle der Miteigentumslösung ( $\$ 92$ Abs. 1 Satz 1 Var. $2 \mathrm{KAGB}^{28}$ ) auch eine dingliche Rechtsposition an den im Sondervermögen befindlichen Vermögensgegenständen. Anteilscheine bewegen sich zwischen Schuldverschreibung und mitgliedschaftlicher Beteiligung (Arg. InvAG und InvKG). Sie repräsentieren einen Bruchteil am Sondervermögen und keine Forderung. Der Anteilschein ist nach h. L. ein eigenständiger Typus und wird deshalb als Wertpapier sui generis qualifiziert. ${ }^{29}$ Umgekehrt dürfte damit in der Neugestaltung des $\$ 95$ KAGB auch die gesetzgeberische Billigung der h. L. gesehen werden können, dass Anteilscheine keine Schuldverschreibungen, sondern Wertpapiere eigener Art sind. ${ }^{30}$ Dann jedoch sind auch elektronische Anteilscheine keine elektronischen Wert-

${ }^{25}$ Begr. RegE eWpG (Fn. 12) S. 41.

26 Siehe zu den möglichen Vorgaben durch RVO noch unter II.9.

27 Siehe dazu auch unter II. 4.

28 Siehe hierzu auch unter II. 2.

29 Emde/Dornseifer/Dreibus/Stabenow, KAGB (2. Aufl. 2019), $\$ 95$ Rn. 7; Moritz/Klebeck/Jesch/Höring, KAGB, Bd.1 (2016), $\$ 95$ Rn. 5; Weitnauer/Boxberger/Anders/Anders, KAGB (3. Aufl. 2021), KAGB, $\$ 95$ Rn. 4; differenzierend neuerdings Assmann/Wallach/ Zetzsche/Zetzsche/Nast, KAGB (2019), $\$ 95$ Rn. 3, 26. Auch $\$ 151$ Nr. 3 StGB, $\$ 2$ Abs. 4 Nr. 2 WpHG und $\$ 1$ Abs. 11 Satz 1 Nr. 5 KWG kennen die Unterscheidung zwischen Anteilen an Investmentvermögen und Schuldverschreibungen.

${ }^{30}$ Hierfür spricht ohnehin bereits $\ 97$ Abs. 2 Satz 2 KAGB, dazu Weitnauer/Boxberger/ Anders/Anders, KAGB (3. Aufl. 2021), KAGB, $\$ 95$ Rn. 4 in Fn. 5. Auch lag dieses Verständnis schon dem RefE (Fn.5) zugrunde, da es andernfalls der Änderung des \95 KAGB nicht bedurft hätte. 
papiere i.S. einer elektronischen Schuldverschreibung, sondern elektronische Anteilscheine eigener Art.

\section{Registerbegebung mit Fiktionswirkung}

Die naturwissenschaftliche contradictio in adiecto, einen unkörperlichen, nicht-dinglichen oder auch materiefreien ${ }^{31}$ elektronischen Datensatz durch einen elektronisch-registerrechtlichen Begebungsakt als Sache zu verkörpern erfolgt gleichwohl unter Rückgriff auf das eWpG im Wege der gesetzlichen Fiktion. ${ }^{32}$ Auch dies ist konsequent, um den rechtstechnischen Rahmen der elektronischen Begebung möglichst einheitlich zu gestalten, ohne den unterschiedlichen Rechtscharakter zwischen Schuldverschreibungen und (papierhaften wie elektronischen) Anteilscheinen außer Acht lassen zu müssen. Mit anderen Worten: Neben den wertpapierrechtlichen Dokumentationsakt der Verbriefung tritt ergänzend, nicht verdrängend der im eWpG geregelte Dokumentationsakt der elektronischen Begebung zur Schaffung wertpapierrechtlicher Fungibilität. ${ }^{33}$

Form und Wirkung des Dokumentationsakts elektronischer Begebung bestimmt sich für elektronische Anteilscheine deshalb nur nach ausgewählten Regelungen des eWpG, die über $\$ 95$ Abs. 3 Satz 1 KAGB zur entsprechenden Anwendung kommen. ${ }^{34}$ Dies erfolgt unter der Maßgabe, dass an die Stelle des „elektronischen Wertpapiers“ der „elektronische Anteilschein“ ( $\$ 95$ Abs.3 Satz 1 Nr. 1 KAGB), an die Stelle der „Emissionsbedingungen“ die „Anlagebedingungen“ ( $\int 95$ Abs. 3 Satz 1 Nr. 2 KAGB $^{35}$ ) und an die Stelle des „Berechtigten“ der „Anleger“ treten ( $\$ 95$ Abs. 3 Satz 1 Nr. 3 KAGB). Diese Verweisungstechnik des KAGB führt dazu, dass das KAGB nicht schweigt, sondern die Begebung elektronischer Anteilschein umfassend regeln. Diese Parallelregelung zum eWpG Kraft gesetzlichen Selektivverweises bewirkt - vorbehaltlich der

31 Vgl. Omlor, RDi 2021, 236, 237: „materiefreies Wertpapier“; erneut Omlor, RDi 2021, 371 mit dem verbildlichen Zusatz „ein Wertpapier ohne Papier“.

32 Plastisch jetzt auch, mit kritischer Einordnung und w. N. Labusen RDi 161, 163: „[...] digitale Produkte den gleichen Gesetzmäßigkeiten unterworfen [...] wie analoge“.

33 Vgl. zum wertpapierrechtlichen Ansatz etwa Baur/Tappen/Mehrkhah/Behme/v. Schweinitz/Schneider-Deters, InvestmentG, Bd. 2 (4. Aufl. 2020), §95 Rn.10a mit Kritik und Nachweisen.

34 Anwendbar sind danach $\$ 2$ Abs. 1 Satz 2, Abs. 2 und 3, die $\int \mathbb{S} 3$ und 4 Abs. 1 Nr. 1, Abs. 2, 4 bis 6, 8 bis 10, die $\iint 6$ bis 8 Abs. 1, Abschnitt 4, $\$ 31$ Abs. 2 Nr. 1 bis 12, Abs. 3 und 4 und $\$ 33$ sowie die $\$ \int 9$ bis 15 mit Ausnahme von $\$ 13$ Abs. 1 Nr. 2 und 3 eWpG. Über die $\$ \$ 15$, $23 \mathrm{eWpG}$ greifen damit auch die VO-Ermächtigungen und auf diesen basierend erlassene VO über Anforderungen an elektronische Wertpapierregister (eWpRV) ein, die seit dem 6. August 2021 als RefE vorliegt, abrufbar über die Internetseite des BMF unter: https://tinyurl. com/294e4f55.

35 Da sich „der Inhalt der Anlagebedingungen ... nicht in sinnvoller Weise auf einige wenige relevante Informationen reduzieren“ lasse, soll auch der Register auf diese Bezug nehmen, so jedenfalls RefE eWpRV (Fn. 34), S. 19. 
Verordnungsermächtigung des $\$ 95$ Abs. 5 KAGB -im Ergebnis eine Komplettregelung, indem die in Bezug genommenen Vorschriften in das KAGB inkorporiert werden.

Zentrale Bestimmungen sind hierbei - wie auch für Schuldverschreibungen aus Sicht des Wertpapierrechts die entsprechende Anwendung des $\$ 2$ Abs. 1 Satz $2 \mathrm{eWp}$, wonach die Verbriefung des Anteilscheins in einer Urkunde durch eine elektronische Begebung unter Eintragung ( $\$ 4 \mathrm{Abs.} 4 \mathrm{eWpG})$ in einem elektronischen Wertpapierregister ( $\$ 4$ Abs. $1 \mathrm{eWpG}$ ) ersetzt wird, ${ }^{36} \$ 2$ Abs. 2 eWpG, der elektronischen Anteilscheinen dieselben Rechtswirkungen wie papierhaften Anteilscheinen beimisst, und $\$ 2 \mathrm{Abs.} 3 \mathrm{eWpG}$, mit der Rechtsfiktion, dass elektronische Anteilscheine als Sachen i.S.d. $\$ 90$ BGB gelten. ${ }^{37}$

Erst diese im KAGB über das eWpG erfolgende dreifache Gleichstellung der elektronischen Begebungsform mit der Verbriefung einerseits $(\$ 2$ Abs. 1 Satz $2 \mathrm{eWpG}$ ) und der elektronischen Speicherung in einem Register unter Fiktion der Sachqualität andererseits ( $\$ 2$ Abs. 3 eWpG) erlaubt aus Sicht des Gesetzgebers die Anwendung sachenrechtlicher Grundsätze auf elektronische Anteilscheine im selben Umfang wie auf Anteilscheine ( $\$ 2$ Abs. $2 \mathrm{eWpG}$ ) aufgrund des durch die Registereintragung gesetzten Rechtsscheins. ${ }^{38}$ Hierdurch gelten weitgehend dieselben depot- und sachenrechtlichen Grundsätze wie für verkörperte Wertpapiere, die sodann durch verschiedene Vorschriften des eWpG zusätzlich abgesichert werden. Die lediglich entsprechende Anwendung über das KAGB führt dabei zu einer Gleichstellung der Rechtswirkungen innerhalb derselben Wertpapiergattung „Anteilschein“, nicht aber über die Gattungen hinweg zu allen (heutigen wie künftigen) elektronischen Wertpapieren. Über diese Gleichstellung mit der Verbriefung hinaus lassen sich aus der Sachfiktion und dem eWpG keine weitergehenden Rechte ableiten, da der positive Verweis des KAGB auf das eWpG die Maximalwirkung der elektronischen Begebung von Anteilscheinen festlegt. ${ }^{39}$ Damit hängen die sachenrechtlichen Wirkungen des elektronischen Begebungsaktes vom durch $\$ 95$ Abs. 3 Satz 1 KAGB in Bezug genommenen Registerrecht des eWpG ab, das erst die Rechtsfiktion des elektronischen Anteilscheins als Wertpapier trägt.

${ }^{36}$ Begr. RegE eWpG (Fn. 12) S. 42.

37 Vgl. zur Fiktionswirkung Omlor, Verfügungen über elektronische Wertpapiere, $\$ 6$, in diesem Band; Bialluch-von Allwörden, Zivil- und prospektrechtliche Aspekte des eWpG-E, RDi 2021, 13, 14 ff.; Omlor, RDi 2021, $236 \mathrm{ff}$.; Omlor, RDi 2021, 371 ff.; Lehmann, NJW 2021, 2318, $2320 \mathrm{Rn} .15 \mathrm{ff}$. Zu zwangsvollstreckungsrechtlichen Implikationen zuletzt Meier, Elektronische Wertpapiere in der Zwangsvollstreckung, MMR 2021, 381, $384 \mathrm{ff}$.

38 Begr. RegE eWpG (Fn.12) S. 43; zu diesen Wirkungen näher Lieder, Eintragung und Publizität elektronischer Wertpapiere, $\$ 5$, in diesem Band; dagegen bspw. Bialluch-von Allwörden, RDi 2021, 13, 14 ff.; Lehmann, BKR 2020, 431, 433, je m. w. N.

39 Gleichwohl ist die Reichweite der Sachfiktion in anderen Rechtsbereichen als dem Wertpapierrecht (bspw. Delikts-, Insolvenz- oder Bereicherungsrecht) schon jetzt streitig, vgl. Omlor, RDi 2021, 236ff.; Lehmann, NJW 2021, 2318, 2320 Rn. 17, je m. w. N.; zum RefE schon Segna, WM 2020, 2301, 2304. 
Hiermit ist schon nach den Gesetzesmaterialien weder die Schaffung eines neuen Wertpapierrechts noch einer neuen Wertpapierart verbunden, ${ }^{40}$ sondern unter Beibehaltung des „numerus clausus“ der Wertpapiere erfolgt nur eine Erweiterung der nutzbaren Formen. Es geht um die Nutzbarmachung vorhandener wertpapierrechtlicher Kategorien und Grundsätze infolge registerrechtlicher, nicht wertpapierrechtlicher Wirkungen. Erst die besondere Transparenz der elektronischen Register, die „das eingetragene Recht [...] genauso sinnlich wahrnehmbar wie eine Urkunde“ macht und „eine für den Rechtsverkehr verlässliche Dokumentation der wesentlichen wertpapierbezogenen Informationen einschließlich der wertpapierrelevanten Transaktionen, die von den einsichtsberechtigten Personen jederzeit eingesehen werden können“ ermöglicht. ${ }^{41}$ Durch die Änderung der genutzten „Speichertechnologie“ des verkörperten Inhalts des Rechts von Papierform zu digital ändert sich nichts an der Rechtsnatur des Wertpapiers. ${ }^{42}$

Entsprechend ändert auch die elektronische Form eines Anteilscheins nichts daran, dass $\$ 95$ Abs. 1 KAGB die Existenz des Anteils als Bündel kollektiv gebundener Rechte und Pflichten voraussetzt. ${ }^{43}$ Dieses Bündel richtet sich bei der sog. Treuhandlösung an KVG und Verwahrstelle (\$9 Abs. 1 Satz 1 Var. 1 KAGB) und umfasst bei der Miteigentumslösung ( $\$ 92$ Abs. 1 Satz 1 Var. 2 KAGB) zudem den Miteigentumsanteil am Sondervermögen. ${ }^{44}$ Wie schon bisher unterscheidet $\$ 95$ Abs. 1 KAGB zwischen den Anteilen an Sondervermögen und körperlichen oder jetzt auch elektronischen Anteilscheinen. ${ }^{45}$ Die Anteile selbst erfahren in $\$ 95$ KAGB auch weiterhin keine Regelung, weshalb sie nur dann und nur in dem Umfang verbrieft oder nach den in Bezug genommenen Regeln des eWpG digitalisiert werden, wie ein Anteil tatsächlich besteht.

Eben deshalb gibt es auch nicht elektronische Anteilscheine „im Sinne von Absatz 1“, wie die Verweisnorm des $₫ 95$ Abs. 3 Satz 1 KAGB (und ebenso fehlerhaft die Verordnungsermächtigung in $\$ 95$ Abs. 5 KAGB) formuliert. Denn \95 Abs. 1 Satz 1 KAGB enthält keine Definition des Anteilscheins oder elektronischen Anteilscheins, sondern deren Rechtsnatur und Entstehung als Wertpapier hängen erst noch von den registerrechtlichen Wirkungen des eWpG ab, die inhaltlich ihre Prägung durch das KAGB erfahren.

${ }^{40}$ Begr. RegE eWpG (Fn. 12) S. 41.

${ }^{41}$ Begr. RegE eWpG (Fn. 12) S. 74.

42 Begr. RegE eWpG (Fn. 12) S. 41.

43 Vgl. statt aller Assmann/Wallach/Zetzsche/Zetzsche/Nast, KAGB (2019), \$95 Rn. 3.

44 Str., wie hier die h.L., die im Anteil und Anteilschein auch eine dingliche Beteiligung erkennt (dazu bspw. Moritz/Klebeck/Jesch/Höring, KAGB, Bd.1 [2016], \$95 Rn.5), während andere eine Verbriefung der dinglichen Rechtsposition ablehnen (so bspw. Assmann/ Wallach/Zetzsche/Zetzsche/Nast, KAGB [2019], \$95 Rn. 3 und 26).

${ }^{45}$ Vgl. zur bisherigen Rechtslage bspw. Moritz/Klebeck/Jesch/Höring, KAGB, Bd.1 (2016), $\$ 95$ Rn. 4. 


\section{Vorrang des $K A G B$ vor dem eWpG}

Inhaltlich sollen seit jeher die Verbriefung und ebenso jetzt das eWpG den erfassten Anteil unberührt lassen, wie nicht nur die soeben beschriebene bloß entsprechende Anwendung des eWpG nach $₫ 95$ Abs. 1 Satz 1 KAGB, sondern jetzt auch $\mathbb{9} 95$ Abs. 3 Satz 2 KAGB belegt. Danach sind Friktionen zwischen eWpG und KAGB stets zugunsten des Letzteren aufzulösen und die Regelungen des eWpG nicht oder jedenfalls nicht in vollem Umfang anzuwenden soweit sich aus den Vorschriften des KAGB etwas anderes ergibt. ${ }^{46}$

Durch diesen Vorrang des KAGB wird einerseits sichergestellt, dass der formale Begebungsakt nach dem eWpG in inhaltliche Ausgestaltung des Anteils durch das KAGB nicht eingreift und ausschließlich zu einer erhöhten Verkehrsfähigkeit führt. Der vom Gesetz verfolgte funktionale Ansatz bedeutet hier, dass die Entmaterialisierung von der Urkunde den spezifischen Regelungen des jeweiligen Wertpapiers Rechnung tragen soll. Andererseits stellt der Vorrang des KAGB sicher, dass das erprobte Schutzinstrumentarium des KAGB durch die Neuerungen des eWp $\mathrm{p}$ keine Einschränkungen erfährt. Denn der Regelungsansatz führt dazu, dass Rechtsfolgen der entmaterialisierten „Verdinglichung“ von Anteilen an Sondervermögen anhand des KAGB und nur subsidiär anhand des eWp zu bestimmen sind.

\section{Keine Anteile an InvAG oder InvKG}

Wie bislang erfasst $\$ 95 \mathrm{KAGB}$ ausschließlich Anteile an Sondervermögen i. S.d. $\$ 1$ Abs. 10 KAGB. ${ }^{47}$ Dies sind inländische offene Investmentvermögen

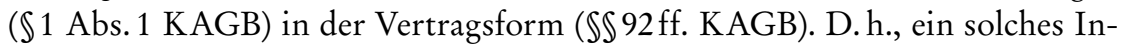
vestmentvermögen in der Form des Sondervermögens wird durch schuldrechtlichen Vertrag zwischen der Kapitalverwaltungsgesellschaft (KVG), Verwahrstelle und den Anlegern begründet und wird als nicht rechtsfähiges Vermögen abgesondert vom Vermögen der KVG verwahrt und verwaltet ( $\$ 92$ Abs. 1 Satz 2 KAGB).

Sondervermögen stellen dabei nur eine Teilmenge aller zulässigen Formen von Investmentvermögen dar, bilden allerdings in der deutschen Praxis auch den größten Teil aller Investmentfonds ( $\$ 3$ KAGB), da solche rechtlich unselbständigen Sondervermögen für OGAWs ( $\$ 1$ Abs. 2 KAGB) sowie für alle Erscheinungsformen von AIFs ( $\$ 1$ Abs. 3 KAGB) gleichermaßen zur Verfügung stehen. ${ }^{48}$

Nicht nach $₫ 95$ Abs. 1 Satz 1 KAGB als elektronischen Anteilscheinen begebbar bleiben folglich Anteile an Investmentgesellschaften i.S.d. $\$ 1$ Abs.11

${ }^{46}$ Ebenso jetzt Omlor, RDi 2021, 371, 372.

47 Zur alten Rechtslage Emde/Dornseifer/Dreibus/Stabenow, KAGB (2. Aufl. 2019), $\$ 95$ Rn. 2.

${ }^{48}$ Emde/Dornseifer/Dreibus/Verfürth/Emde, KAGB (2. Aufl. 2019), $\$ 1$ Rn. 165. 
KAGB als weitere zulässige Erscheinungsformen von Investmentvermögen. ${ }^{49}$ Diese dürfen nur in der Satzungsform als Investmentaktiengesellschaft (InvAG, \$S $108 \mathrm{ff}$. und 140ff. KAGB) oder in Gesellschaftsvertragsform als Investmentkommanditgesellschaft (InvKG, $\int \mathbb{S} 124 \mathrm{ff}$. und $149 \mathrm{ff}$. KAGB) begründet werden, für welche die jeweiligen gesellschaftsrechtlichen Normen des AktG und des HGB modifiziert durch das KAGB Anwendung finden. Solange daher die Regelung des eWpG Aktien und andere Gesellschaftsanteile bewusst ausklammert, solange bleiben trotz der Erweiterungen des neuen $\$ 95$ KAGB auch bei den Investmentfonds elektronische Aktien oder Gesellschaftsanteile außen vor. ${ }^{50}$

\section{Inhaberfondsanteile}

Die Neufassung des $\int 95$ Abs. 1 Satz 2 KAGB schränkt in Parallele zur Einschränkung des Anwendungsbereichs in $\$ 1 \mathrm{eWpG}$ auf Inhaberschuldverschreibungen das Wahlrecht zur Begebungsform auf Anteile an Sondervermögen dahingehend ein, dass die Anteile auf den Inhaber lauten müssen. ${ }^{51}$ Anteilscheine auf den Namen müssen weiterhin verbrieft, also papierhaft verkörpert werden. Indes kann auch dies nicht wirklich erstaunen, sind doch bei elektronischen Anteilscheinen ohnehin die Inhaber entweder als (materiell) Berechtigte oder als nichtberechtigte treuhänderische Sammelverwahrer im dafür vorgesehenen Register zu vermerken ( $\$ 95$ Abs. 3 Satz 1 KAGB i. V.m. $\$ 3, \$ 8$ Abs. 1 und $\$ 13$ Abs. 1 Nr. 6 eWpG $\left.{ }^{52}\right)$.

Einzelanteile an Sondervermögen - soweit sie wegen $₫ 97$ Abs. 1 Satz 2 KAGB überhaupt zulässig sind ${ }^{53}$ - stellen so besehen stets elektronische Namensanteile dar, denn auch jede weitere Übertragung des Anteils bedarf der Eintragung des neuen Inhabers als Berechtigten in das Register. ${ }^{54}$ Es gilt für elektronische

$49 \mathrm{Vgl}$. zur Unterscheidung zwischen materiellen und formal zulässigen Investmentvermögen Eckbold/Balzer, in: Assmann/Schütze/Buck-Heeb, Handbuch des Kapitalanlagerechts (5. Aufl. 2020), $\$ 22$ Rn. 3, 7 m. w. N.

50 Kritisch aus allgemeiner gesellschaftsrechtlicher Sicht hierzu Möslein, Elektronische Gesellschaftsanteile, $\mathbb{8} 8$, in diesem Band; Reger/Langheld/Haagen, Elektronische Aktien, RDi 2021, 83, 87 ff. m. w. N. Einordnung wie hier jetzt bei Omlor, RDi 2021, 371, 372.

51 Zur Reichweite der „Inhaberschuldverschreibungen“ siehe Lebmann, Wertpapierarten, $₫ 3$ II, in diesem Band.

52 Zukünftig über die nach $\$ 95$ Abs. $5 \mathrm{KAGB}$ mögl. RV ist dies auch aufgrund $\$ 17 \mathrm{Abs} .1$ Nr. 6 eWpG denkbar.

53 Siehe sogleich unter II.6; dagegen offenbar generell für Zentralregisterwertpapiere Mittwoch, WM 2021 375, 379 („[...] ausschließlich im Wege der Sammelverwahrung [...]“) wegen $\$ 6$ Abs. 1 Satz 1 eWpG.

54 Sei es beim sammeleingetragenen Zentralregisteranteil nach sachen- und depotrechtlichen Grundsätzen des Effektengiroverkehrs oder bei Kryptofondsanteilen nach \95 Abs. 4 KAGB i. V.m. der Rechtsverordnung und $\$ 25$ Abs. 1, $\$ 4$ Abs. 8 eWpG. Vgl. dazu Mittwoch, WM 2021 375, 380f.; Meier, RDi 2021, 1 ff. m. w. N. 
Wertpapiere in Einzeleintragung der Grundsatz „keine Verfügung außerhalb des Registers“.

Sammelanteile sind in papierhafter wie auch in elektronischer Form immer Inhaberfondsanteile, treuhänderisch durch eine Wertpapiersammelbank oder einen Verwahrer verwahrt ( $\$ 95$ Abs. 3 Satz 1 KAGB i. V. m. $\int \mathbb{S} 8$ Abs. 1, 9 Abs. 2 eWpG). Findet sich hier auch nicht der Name des Berechtigten (i.S.d. materiellen Rechtsinhabers gem.\$95 Abs. 3 Satz 1 KAGB i. V.m. $\$ 3$ Abs. 2 eWpG) im Register, so jedoch der Name dessen, der für die Berechtigten formal als Inhaber die elektronischen Anteile hält ( $\$ 95$ Abs. 3 Satz 1 KAGB i. V.m. $\mathbb{3}$ Abs. 1 eWpG).

\section{Sammel-nicht Einzeleintragung}

Während $\ 95$ Abs. 2 Satz 1 KAGB notwendigerweise die bisherige Regelung des $\$ 95$ Abs. 1 Satz 3 KAGB a.F. beibehält, dass Inhaberanteilscheine in einer Sammelurkunde zu verbriefen sind und der Anspruch auf Einzelverbriefung auszuschließen ist, verweist der neue $\$ 95$ Abs. 3 Satz 1 KAGB für elektronische Anteilscheine uneingeschränkt auf eine entsprechende Anwendung von $\ 8$ eWpG. Dies ließe bei wortlautgetreuer Anwendung sowohl Sammel- als auch Einzeleintragungen elektronischer Anteilscheine zu, zumal es über $\$ 95 \mathrm{Abs.} 3$ Satz 1 KAGB i. V.m. $\$ 6$ Abs. 1 Satz 2 eWpG möglich erscheint, dass die Kapitalverwaltungsgesellschaft den Anlegern sogar einen Anspruch auf Einzelverbriefung in Wertpapierurkunden gewährt.

Letzteres ist gleichwohl wegen $₫ 97$ Abs. 1 Satz 2 KAGB zweifelhaft. Denn danach sind (ohne Unterscheidung zwischen papierhaft und elektronisch) Inhaberanteilscheine stets einer Wertpapiersammelbank oder dieser gleichwertigen Verwahrstelle (CSDR, ${ }^{55} \$ 5$ Abs. 4 Satz 1 DepotG) zur Sammelverwahrung anzuvertrauen. Dies begründet im Zusammenspiel mit dem Ausschluss des Anspruchs auf Einzelverbriefung das sog. Zwangsgiro. ${ }^{56}$ Werden hiervon auch elektronische (Inhaber-)Anteilscheine erfasst, dann zwingt der Vorrang des KAGB nach $\$ 95$ Abs. 3 Satz 2 KAGB, die Wahl der Einzeleintragungen auszuschließen. Hierfür spricht auch $\$ 12$ Abs. $3 \mathrm{eWpG}$, wonach nur Zentralregisterwertpapiere in den Effektengiro einbezogen werden, deren Zentrale Register von einer als Inhaber eingetragenen Wertpapiersammelbank geführt werden. ${ }^{57}$

Zwingend erforderlich erscheint dies wiederum nicht. Anlass für das Zwangsgiro ist die Vermeidung effektiver Stücke, um die Erleichterungen von

55 VO (EU) Nr. 909/2014 des Europäischen Parlaments und des Rates vom 23. Juli 2014 zur Verbesserung der Wertpapierlieferungen und -abrechnungen in der Europäischen Union und über Zentralverwahrer sowie zur Änderung der Richtlinien 98/26/EG und 2014/65/EU und der Verordnung (EU) Nr. 236/2012 (ABl. EU Nr. L 257 vom 28.8.2014, S. 1).

56 Zum Hintergrund Assmann/Wallach/Zetzsche/Zetzsche/Nast, KAGB (2019), $\$ 95$ Rn. 9.

57 Vgl. allg. auch Wieneke/Kunz, NZG 2021, 316, 320. 
Meldepflichten nach zum US-amerikanischen Foreign Account Tax Compliance Act geschlossenen Abkommen für Investmentfonds in Anspruch nehmen zu können. ${ }^{58}$ Denn dies setzt eine geschlossene Depotbankkette vom Investmentfonds zu den Anlegern voraus, die bei einer Einzelverbriefung und dem damit möglichen Umlauf der einzelnen Urkunde nicht sichergestellt ist. ${ }^{59}$ Dieses Risiko stellt sich bei der elektronischen Anteilscheinform indes nicht. Diese sind immer registerverwahrt. Über die Registrierung ist die Identifizierung der Inhaberschaft bei der Einzeleintragung unmittelbar oder bei der Sammeleintragung mittelbar über dieselbe geschlossene und nachvollziehbare (elektronische) depotrechtlich hergestellte Kette wie im Falle des Zwangsgiro sichergestellt ( $\$ 95$ Abs. 3 Satz 1 KAGB i. V.m. $\$ 9$ eWpG).

Für den theoretisch möglichen, schon jetzt absehbar nicht praxisrelevanten Fall des Wechsels vom elektronischen Anteilschein zu einem papierhaften Anteilschein ist im Grundsatz der Anspruch auf Ausreichung einzelner Wertpapierurkunden ausgeschlossen ( $\mathbb{9} 95$ Abs. 3 Satz 1 KAGB i.V.m. $\$ 6$ Abs. 1 Satz 1 eWpG). Zwar scheinen die Anlagebedingungen des elektronischen Anteilscheins einen solchen Anspruch ausdrücklich vorsehen zu können, denn $\$ 95$ Abs. 3 Satz 1 KAGB scheint auch auf $\$ 6$ Abs. 1 Satz 2 eWpG zu verweisen. Doch geht dieser Verweis jedenfalls im Falle der nachträglichen Verbriefung ins Leere. Der Vorrang des KAGB gemäß der Anordnung in $₫ 95$ Abs. 3 Satz 2 KAGB führt dazu, dass für den papierhaften Anteilschein auch bei einem späteren Umtausch in ein Wertpapier bei Inhaberfondsanteilscheinen die Anordnung des Zwangsgiro nach $\$ 95$ Abs. 2 Satz 1 KAGB vorrangig wieder eingreift.

\section{Anteilsklassen und Teilsondervermögen}

Das Wahlrecht elektronische Inhaberfondsanteilscheine an Sondervermögen zu begeben, gilt nicht nur für einzelne (Investment-)Sondervermögen in ihrer Gesamtheit, sondern auch für verschiedene Anteilsklassen desselben Sondervermögens ( $\mathbb{S} 96$ Abs. $1 \mathrm{KAGB}$ ) oder Teilsondervermögen ( $\$ 96$ Abs. $2 \mathrm{KAGB}$ ). Hier kann das Wahlrecht sogar unterschiedlich ausgeübt werden. ${ }^{60}$ Dies folgt aus dem gesetzlichen Ansatz, papierhafte und elektronische Anteilscheine gem. \$95 Abs. 3 Satz 1 KAGB i. V. m. \$6 eWpG als austauschbar anzusehen, da diese nach $\$ 95$ Abs. 3 Satz 1 KAGB i.V.m. $\$ 2$ Abs. 2 eWpG stets dieselben Rechtswirkungen entfalten. Konsequenterweise sind sogar Mischbestände teils aus ei-

58 Siehe dazu Abkommen zwischen der Bundesrepublik Deutschland und den Vereinigten Staaten von Amerika zur Förderung der Steuerehrlichkeit bei internationalen Sachverhalten und hinsichtlich der als Gesetz über die Steuerehrlichkeit bezüglich Auslandskonten bekannten US-amerikanischen Informations- und Meldebestimmungen vom 31.5.2013, BGBl. II 2013, S. 1363.

59 Begr. RegE OGAW-V-UmsG v. 18.11.2015, BT-Drs. 18/6744, S. 55 f.

60 Vgl. zum bisherigen Recht bspw. Moritz/Klebeck/Jesch/Höring, KAGB, Bd.1 (2016), $\$ 95 \mathrm{Rn} .2$. 
ner Sammeleintragung und teils aus mittels Urkunde begebenen Wertpapieren oder Wertpapieren in Einzeleintragung im selben Register möglich, die als ein einheitlicher Sammelbestand gelten, wenn dies im Register zur Sammeleintragung vermerkt ist ( $\$ 95$ Abs. 3 Satz 1 KAGB i. V. m. $\$ 9$ Abs. 3 eWpG).

\section{Zentralregisterfondsanteile}

Die weiteren von $\$ 95$ Abs. 3 Satz 1 KAGB in Bezug genommenen Regelungen dienen vornehmlich der Ausgestaltung der Art und Weise des die „Verbriefung“ ersetzenden Dokumentationsakts der „elektronischen Begebung“ und den Dokumentationsanforderungen an Verfügungen über elektronische Anteilscheine. Hierbei beschränkt sich der Verweis in \95 Abs. 3 Satz 1 KAGB auf die Begebung von elektronischen Anteilscheinen als Zentralregisterwertpapiere i.S.d. $\$ 4$ Abs. $2 \mathrm{eWpG}$, so dass über $\$ 95$ Abs. 3 Satz 1 KAGB Zentralregisteranteilscheine begeben werden können. ${ }^{61}$

Diese weisen eine deutliche Nähe zu den bekannten Formen der girosammelverwahrten Anteilscheine oder allgemeinen Wertpapieren auf. Erneut sind sie bei wortlautgetreuer Umsetzung des Verweises in das eWpG in einem durch eine Wertpapiersammelbank (\$95 Abs. 3 Satz 1 KAGB i.V.m. $\$ 4$ Abs. 5 eWpG) oder eine Depotbank ( $\$ 95$ Abs. 3 Satz 1 KAGB i. V. m. $\$ 4$ Abs. 6 eWpG) zu füh-

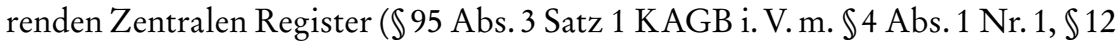
Abs. 2 eWpG) mit den dazu erforderlichen Angaben einzutragen ( $\$ 95$ Abs. 3 Satz 1 KAGB i. V.m. $\ 4$ Abs. 4 und 5, $\$ 13 \mathrm{eWpG}$ ). Insoweit hat der RegE den RefE dahingehend abgeändert, dass jedenfalls Inhaberschuldverschreibungen als Zentralregisterwertpapiere auch der Hausverwahrung zugänglich sind. ${ }^{62}$ Die Möglichkeit der Führung des Zentralen Registers durch eine Depotbank wirft allerdings die Frage auf, ob tatsächlich auch elektronische Anteilscheine anders als ihr papierhaftes Pendant bei entsprechender Ermächtigung durch die Kapitalverwaltungsgesellschaft durch eine Depotbank statt wie im Falle des $\$ 97$ Abs. 1 Satz 2 KAGB nur von einer Wertpapiersammelbank (und gleichwertig, CSDR, ${ }^{63} \$ 5$ Abs. 4 Satz 1 DepotG) sammelverwahrt werden können, da die Registerführung hier der Sammelverwahrung gleichsteht. Dies ist zu verneinen, wenn $\$ 97$ Abs. 1 Satz 2 KAGB auch elektronische Anteilscheine erfasst.

${ }^{61} \mathrm{Zu}$ den Möglichkeiten Kryptofondsanteile über $\$ 95$ Abs. 5 KAGB zu begeben sogleich unter II.9.

62 Wieneke/Kunz, NZG 2021, 316, 320; vgl. schon zum RefE auch Segna, WM 2020, 2301, 2305.

63 VO (EU) Nr. 909/2014 des Europäischen Parlaments und des Rates vom 23. Juli 2014 zur Verbesserung der Wertpapierlieferungen und -abrechnungen in der Europäischen Union und über Zentralverwahrer sowie zur Änderung der Richtlinien 98/26/EG und 2014/65/EU und der Verordnung (EU) Nr. 236/2012 (ABl. EU Nr. L 257 vom 28.8.2014, S. 1) - Central Securities $\underline{\text { Depositories }}$ Regulation. 
Ebenfalls nicht ausdrücklich geklärt wird durch $\$ 95 \mathrm{KAGB}$, wer die Ausgabe elektronischer Anteilscheine vorzunehmen hat. Grundsätzlich obliegt die Ausgabe der Anteile an Sondervermögen der Verwahrstelle (\$S 71 Abs. 1, 83 Abs. 1 Nr. 1 KAGB). Anteilscheine sind dabei gem. $\ 95$ Abs. 2 Satz 4 KAGB weiterhin von der Kapitalverwaltungsgesellschaft und der Verwahrstelle zu unterzeichnen. Hierdurch soll sichergestellt werden, dass nur unter Mitwirkung der Verwahrstelle Anteilscheine wirksam in Umlauf gebracht werden. ${ }^{64}$ Zugleich wird darin die Dokumentation durch die Verwahrstelle gesehen, dass diese ihre Rechte und Pflichten aus dem Investment- und häufig auch des zweiseitigen Begebungsvertrages annimmt. ${ }^{65}$ Es fragt sich deshalb, wie der Skripturakt der Eintragung in einem Zentralen Register nach $\$ 95$ Abs. 3 Satz 1 KAGB i. V.m. $\$ 2$ Abs. 2 eWpG gemeinschaftlich durch die Kapitalverwaltungsgesellschaft und die Verwahrstelle zu bewirken sind? Wer ist als Emittent nach $\$ 95$ Abs. 3 Satz 1 KAGB i. V.m. \13 Abs. 1 Nr. 4 eWpG im Zentralen Register einzutragen?

Da nach dem KAGB die Ausgabe eines Anteilscheins eine Erfüllungshandlung der Kapitalverwaltungsgesellschaft ist dürfte auch bei elektronischer Begebung eine Pflicht der Kapitalanlagegesellschaft anzunehmen sein, mit dieser Tätigkeit die Verwahrstelle zu beauftragen. Im Rahmen dieser Beauftragung handelt die Verwahrstelle im Namen und für Rechnung der Kapitalverwaltungsgesellschaft. Unter entsprechender Anwendung des eWpG spricht danach mehr dafür, dass die Kapitalverwaltungsgesellschaft im Zusammenwirken mit der Verwahrstelle als Emittent die elektronische Begebung durch Eintragung in das Zentrale Register zu bewirken hat, zumal nach h. L. die fehlerhafte Begebung eines Anteilscheins zu dessen Unwirksamkeit nach \$134 BGB führt.

Die Begründung des RegE liefert keine Gründe für die Einschränkung des \95 Abs. 3 Satz 1 KAGB auf Zentralregisteranteilscheine mit, ${ }^{66}$ doch die Beschlussempfehlung und der Bericht des Finanzausschusses hat diese nachgereicht. Danach ist die Einführung von „Kryptofondsanteilen“ zurückgestellt, da weitere Prüfungen erforderlich seien, wie den Besonderheiten von Anteilen an Investmentfonds bei Eintragung in dezentrale Kryptowertpapierregister Rechnung getragen werden könne. Dies beträfe insbesondere die Rechtsstellung der Verwahrstelle. ${ }^{67}$

Diese Beschränkung elektronischer Anteilscheine auf Zentrale Register erscheint durchaus nachvollziehbar. Investmentrecht, insbesondere im Bereich der OGAWs und anderer Formen der AIF-Publikumsfonds ist geprägt von besonderer formaler Ausgestaltung und staatlicher Überwachung. Kryptowertpapieregister und die damit verbundenen Informationstechnologien haben noch

${ }^{64}$ Moritz/Klebeck/Jesch/Höring, KAGB, Bd. 1 (2016), \$95 Rn. 14 m. w. N.

${ }^{65} \mathrm{Baur} /$ Tappen/Mehrkhah/Behme/v. Schweinitz/Schneider-Deters, InvestmentG, Bd. 2

(4. Aufl. 2020), $\$ 95$ Rn. 45 m.w. N.

${ }_{66}$ Begr. RegE eWpG (Fn. 12), S. 85 f.

67 Beschlussempfehlung und Bericht des Finanzausschusses eWpG (Fn. 14), S. 61. 
keine breite Erprobung erfahren. Technische Standards bilden sich noch aus und insbesondere fehlt es derzeit an Überwachungsmöglichkeiten durch die Aufsicht.

\section{Krypto(register)fondsanteile}

Die damit verbundenen Themenstellungen sieht entgegen dem RefE und den ursprünglichen RegE der Finanzausschuss offenbar aber als schnell und ohne weitere Beratung durch den Bundestag als lösbar an. Deshalb wurde auf den sprichwörtlichen „letzten Metern“ des Gesetzgebungsverfahrens mit der Beschlussempfehlung des Finanzausschusses an $\$ 95$ KAGB ein neuer Absatz 5 angefügt. Dieser enthält eine gemeinsame Ermächtigung von BMF und BMJV durch Rechtsverordnung, die nicht der Zustimmung des Bundesrates bedarf, die Voraussetzungen für die spätere Ausweitung des Anwendungsbereichs zur Einführung von „Kryptofondsanteilen“, also elektronischen Investmentfondsanteilen, die in ein dezentrales Kryptowertpapierregister eingetragen sind, zu ermöglichen. ${ }^{68}$ Über eine Verordnung soll danach die „entsprechende oder teilweise entsprechende“ Anwendung derjenigen Regelungen des eWpG „auf elektronische Anteilscheine im Sinne von“ \$95 Abs. 1 KAGB angeordnet werden können, die Kryptowertpapiere betreffen, ${ }^{69}$ also Kryptowertpapiere in der Ausprägung von Kryptoanteilscheinen. ${ }^{70}$

Voraussetzung soll nach dem Bericht des Finanzausschusses sein, dass rechtliche Anforderungen an Kryptoanteilscheine angemessen ausgestaltet und den Anforderungen des KAGB, insbesondere auch im Hinblick auf die Wahrung des Anlegerschutzes, Rechnung getragen wird. Zugleich sollen durch Ausgestaltung der Rechtsverordnung die Anforderungen an Kryptowertpapierregister an die Erfordernisse von Kryptoanteilscheinen angepasst werden können. Deshalb sieht $\$ 95$ Abs. 5 Satz 3 KAGB vor, dass von den Vorgaben der in Bezug genommenen Regelungen des eWpG in der Rechtsverordnung Abweichungen bestimmt werden können, insbesondere für die Regelungen betreffend die Verwahrstelle, soweit dies aufgrund der Besonderheiten bei elektronischen Anteilscheinen erforderlich ist.

Allerdings stellt $\$ 95$ Abs. 5 KAGB anders als der Verweis auf das eWpG in \95 Abs. 3 KAGB nicht explizit klar, dass die Regelungen des KAGB vor dem eWpG und auch der Rechtsverordnung Vorrang haben. Doch systematisch und nach seinem Sinn und Zweck ist die Verordnungsermächtigung in $\$ 95$ Abs. 5 KAGB lediglich als Verlängerung des $₫ 95$ Abs. 3 Satz 1 KAGB zu begreifen.

68 Beschlussempfehlung und Bericht des Finanzausschusses eWpG (Fn. 14), S. 61.

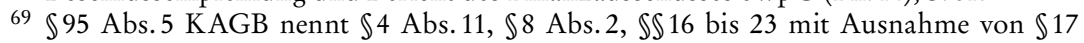
Abs. 1 Nrn. 2 und 3, sowie die $\iint 30,31$ Abs. 1 und 2 Nrn. 13 bis 15 eWpG.

70 Beschlussempfehlung und Bericht des Finanzausschusses eWpG (Fn. 14), S.61, sprechen von „Kryptoanteilss cheinen“. Da das Gesetz aber im Übrigen vom Anteilschein spricht, wird hier die Schreibweise Kryptoanteilschein verwendet. 
Dieses Verständnis ist wichtig, da damit die durch Rechtsverordnung zugelassenen Kryptoanteilscheine gleichermaßen wie Zentralregisteranteilscheine der Beschränkung des $\$ 95$ Abs. 3 Satz 2 KAGB unterliegen. Die Verordnungsermächtigung des $₫ 95$ Abs. 5 KAGB erlaubt keine Abweichungen vom KAGB, sondern nur eine Erweiterung der zulässigen Formen elektronischer Anteilscheine. Die inhärenten Begrenzungen des KAGB bleiben somit unangetastet.

Dies erscheint schon deshalb zwingend, da ohnehin bezweifelt werden kann, ob die Verordnungsermächtigung den Anforderungen des Art. 80 GG genügt. Danach ist zu verlangen, dass Inhalt, Zweck und Ausmaß der erteilten Ermächtigung im KAGB bestimmt werden (Art. 80 Abs. 1 Satz 2 GG). $\$ 95$ Abs. 4 KAGB und das ihm zugrundeliegende Gesetzgebungsverfahren bringt klar zum Ausdruck, dass die Auswirkungen der Zulassung von Kryptofondsanteilen keineswegs durchdacht und noch zahlreiche Fragen zu klären sind. Dass hier Reichweite und inhaltlichen Anforderungen hinreichend bestimmt sind, darf bezweifelt werden. Dann jedoch ist jedenfalls zu fordern, dass die Verordnung sich nicht in Widerspruch zu Vorgaben des KAGB setzen darf.

Hierdurch ist es möglich, zunächst sämtliche Regelungen, die elektronische Wertpapiere in Kryptowertpapierregistern auf einer DLT zulassen, auszuklammern. Es bleibt indes ein „Hintertürchen“ offen, um über die Verordnungsermächtigung des $\ 95$ Abs. 5 KAGB zu einem späteren Zeitpunkt Kryptofondsanteile zu ermöglich. Gleichwohl belegt auch dieses Regelungskonzept, worum es dem Gesetz geht: Das eWpG ist nur auf Schuldverschreibungen bezogen und jede andere Öffnung für elektronische Wertpapiere soll für den Einzelfall erwogen werden.

\section{Erste Bewertung}

Wird eine erste Bewertung der Öffnung des KAGB für digitale Anteile an Sondervermögen versucht, ist zunächst zu begrüßen, dass auch das KAGB mit den technischen Entwicklungen der Digitalisierung Schritt halten soll. Ein erster Schritt in die richtige Richtung ist getan, auch wenn über den regulatorischen Ansatz gestritten werden kann. Hier wie zum eWpG und seinen elektronischen Wertpapieren fragt es sich aber, ob „Insellösungen“ zielführend sind.

Das eWpG und auch dessen Inkorporation in das KAGB führt zu zahlreichen Fragestellungen, die bei dem erforderlichen Voranschreiten zu einem „buchungsbasierten Rechts der Kapitalmarkt-Wertpapiere“ erneut zu beantworten sein werden. Ob insoweit die „Brückenlösung“ des wertpapier-sachenrechtliche Ansatzes hier zu weitergehenden und nutzbar zu machenden Erkenntnissen führt, bleibt abzuwarten, zumal schon das eWpG in seinem Anwendungsbereich viele Fragen unbeantwortet lässt. 
Wenn nun jedoch der Weg über die Brückenlösung eingeschlagen ist, dann ist zu begrüßen, dass der Gesetzgeber sich zunächst aufZentralregisterfondsanteile beschränkt. Diese weisen eine erkennbare Nähe zur zumindest praxisbewährten Girosammelverwahrung auf, die im Wesentlichen schon bislang „entmaterialisiert" abgewickelt wurde. Etwaige entstehende Friktionen dürften daher beherrschbar bleiben. Hierfür stellt das KAGB durch die lediglich entsprechende Anwendung des eWpG ein taugliches rechtliches Werkzeug zur Verfügung, um digital-registrierte Investmentfondsanteile umzusetzen. Die Einführung von Kryptofondsanteilen wirft indes im komplizierten Geflecht investmentrechtlicher Beziehungen weitere Fragen auf, die sorgfältig bedacht werden sollten. Deshalb ist es zweifelhaft, wenn eine Regelung der Kryptofondsanteile durch Rechtsverordnung ermöglicht wird. Der Bereich der Publikumsfonds mag insoweit nicht das geeignete Erprobungsfeld neuer Technologien sein. 


\section{Autorenverzeichnis}

Buttlar, Julia von, Dr. rer. pol., LL.M. (Duke), Regierungsdirektorin, Bundesanstalt für Finanzdienstleistungsaufsicht, Frankfurt a.M.

Eckhold, Thomas, Dr. iur., LL.M. (Warwick), Partner in der Kanzlei Sernetz Schäfer Rechtsanwälte Partnerschaft mbB, Düsseldorf.

Einsele, Dorothee, Dr. iur., o. Professorin für Bürgerliches Recht, Handelsrecht einschließlich des Bank- und Wertpapierrechts, Internationales Privatrecht sowie Rechtsvergleichung, Christian-Albrechts Universität zu Kiel.

Grundmann, Stefan, Dr. iur., Dr. phil., LL.M. (Berkeley), o. Professor für Bürgerliches Recht, Deutsches, Europäisches und Internationales Privat- und Wirtschaftsrecht, Humboldt-Universität zu Berlin, sowie Mitgründer und derzeit Präsident der Society of European Contract Law, der internationalen Fachvereinigung für Europäisches Vertragsrecht.

Habersack, Mathias, Dr. iur., o. Professor für Bürgerliches Recht und Unternehmensrecht, Ludwig-Maximilians-Universität München.

Hirte, Heribert, Dr. iur., LL.M. (Berkeley), Mitglied des Deutschen Bundestages, o. Professor für Zivilrecht, Bürgerliches Recht, Handels- und Gesellschaftsrecht, Wirtschaftsrecht, Universität Hamburg.

Lambrecht, Christine, Mitglied des Deutschen Bundestages und Bundesministerin für Justiz und Verbraucherschutz sowie für Familie, Senioren und Jugend.

Lehmann, Matthias, Dr. iur., D.E A. (Paris II), LL.M., J.S.D. (Columbia), o. Professor für Europarecht, Internationales Recht und Rechtsvergleichung, Universität Wien.

Lieder, Jan, Dr. iur., LL.M. (Havard), o. Professor für Bürgerliches Recht, Handels- und Wirtschaftsrecht, Direktor der Abteilung Wirtschaftsrecht des Instituts für Wirtschaftsrecht, Arbeits- und Sozialrecht, Albert-Ludwigs-Universität Freiburg.

Monnerat, Lucien, Studierender in Assistenzfunktion (Professur ZellwegerGutknecht), Universität Basel. 
Möslein, Florian, Dr. iur., Dipl.-Kfm., LL.M. (London), o. Professor für Bürgerliches Recht, Deutsches und Europäisches Wirtschaftsrecht, Gründungsdirektor des Instituts für das Recht der Digitalisierung (IRDi), Philipps-Universität Marburg.

Omlor, Sebastian, Dr. iur., LL.M. (NYU), LL.M. Eur., o. Professor für Bürgerliches Recht, Handels- und Wirtschaftsrecht, Bankrecht sowie Rechtsvergleichung, Gründungsdirektor des Instituts für das Recht der Digitalisierung (IRDi), Philipps-Universität Marburg.

Zellweger-Gutknecht, Corinne, Dr. iur., o. Professorin für Privatrecht und Wirtschaftsrecht, Universität Basel. 